Modelación geoestadística de medios geológicos naturalmente fracturados

Tesis Doctoral

\title{
Autor
}

Pablo Romanazzi

Director

Eduardo Cassiraga 


\section{Modelación geoestadística de medios geológicos naturalmente fracturados}

Tesis Doctoral

\begin{tabular}{|c|c|l|c|c|}
\hline Rev. & Fecha & Descripción de la/s enmienda/s & Edición & Revisión \\
\hline A & $17 / 05 / 2017$ & Texto completo - versión 1 (de emisión) & PGR & EFC \\
\hline B & $31 / 05 / 2017$ & Texto mejorado - versión corregida & PGR & EFC \\
\hline C & $03 / 07 / 2017$ & Texto mejorado - correcciones s/Guardiola y Kruse (eval.) & PGR & EFC \\
\hline D & $05 / 09 / 2017$ & Texto corregido definitivo - versión 2 & PGR & EFC \\
\hline E & $30 / 09 / 2017$ & Texto corregido definitivo - versión 3 & PGR & EFC \\
\hline
\end{tabular}


Ficha Técnica

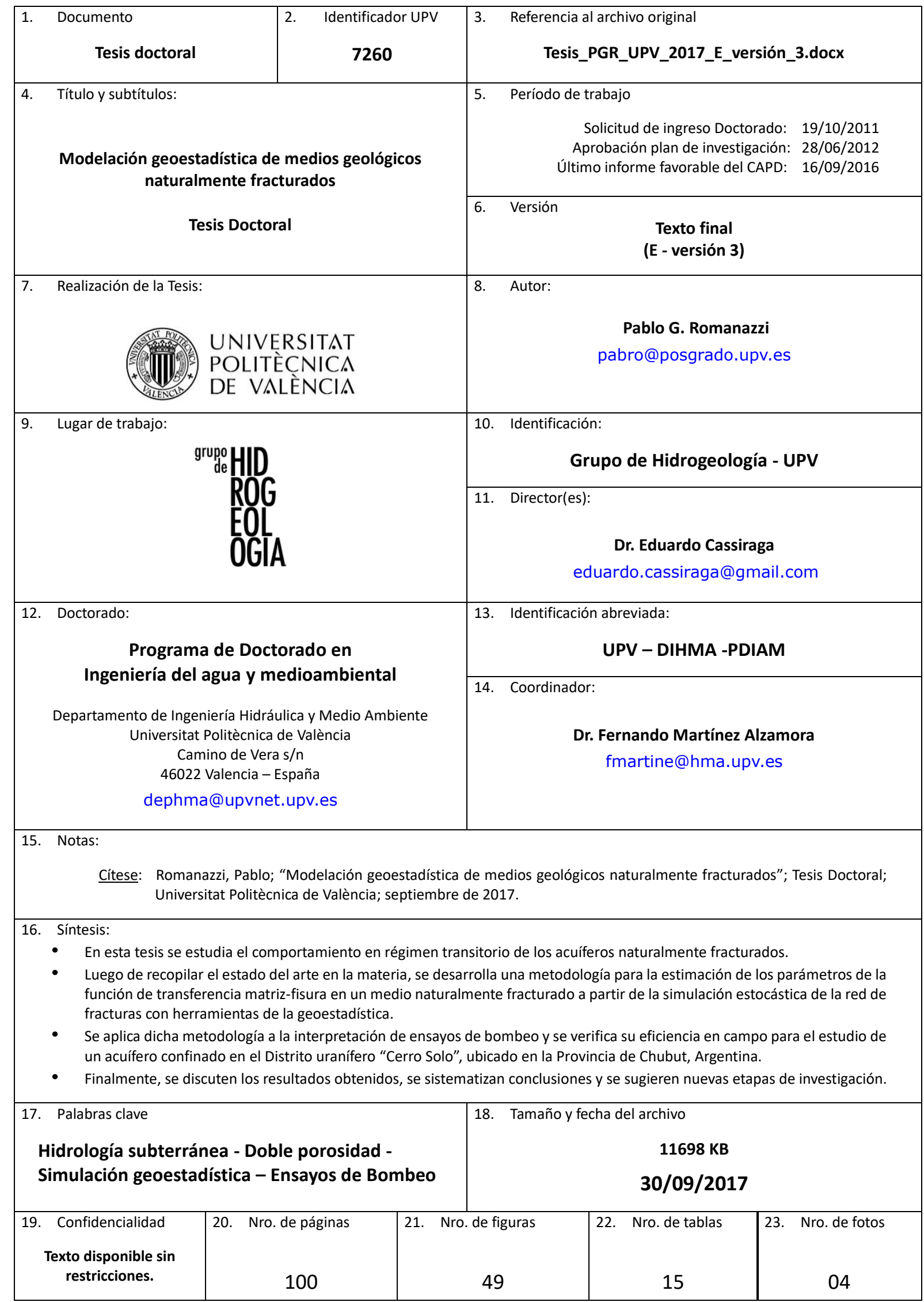


"Cambiaré de opinión tantas veces y tan a menudo como adquiera conocimientos nuevos, el día que aperciba que mi cerebro ha dejado de ser apto para esos cambios, dejaré de trabajar. Compadezco de todo corazón a todos los que después de haber adquirido y expresado una opinión, no pueden abandonarla nunca más".

Florentino Ameghino

(científico autodidacta argentino, 1854-1911)

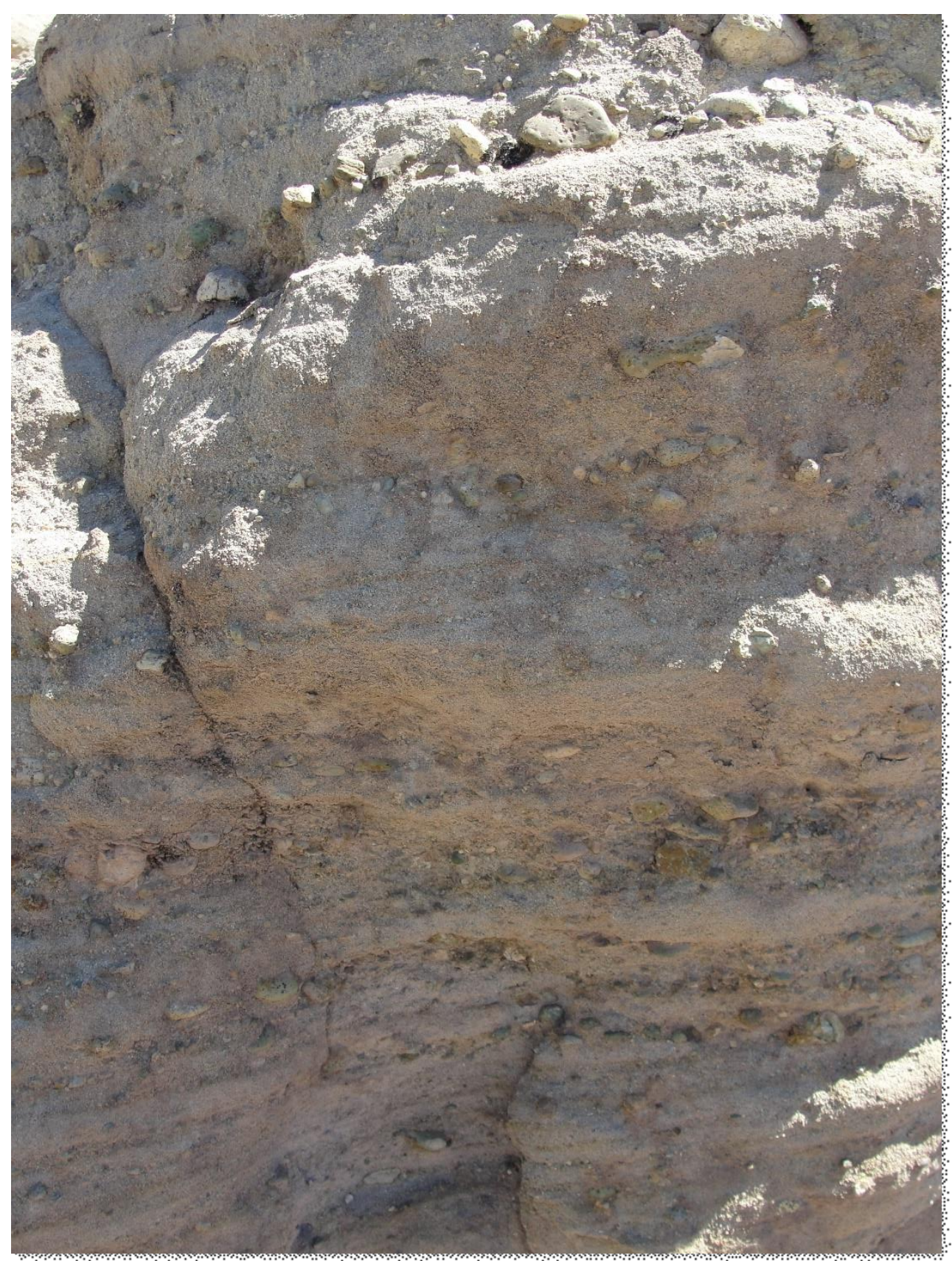

Foto de la portada: Afloramiento del acuífero cretácico Arroyo del Pajarito, Paso de Indios, Chubut, Argentina. 


\section{RESUMEN}

La modelación del flujo en medios geológicos naturalmente fracturados depende principalmente de la composición estructural de la relación matriz-fractura y de la forma en que se aproxime el intercambio de fluido entre ambas fases.

Las dos características mencionadas (composición estructural de la doble porosidad y la función de transferencia de flujo) incorporan el mayor grado de incertidumbre en la modelación de los estados transitorios generados por bombeo.

Para poder mejorar la interpretación del ensayo de bombeo y con el objetivo último de obtener parámetros característicos confiables del acuífero (tales como la conductividad y el coeficiente de almacenamiento para ambas fases), la metodología propuesta en esta tesis aprovecha las herramientas que brinda la geoestadística para simular realizaciones de campos de fisuras cuyo ensamble permite a su vez estimar los parámetros de forma de la función de transferencia matrizfisura (FTMF).

En efecto, los métodos clásicos disponibles para interpretar resultados de ensayos de bombeo en acuíferos heterogéneos, en especial aquellos que se presentan naturalmente fracturados y con una compleja relación entre las dos componentes porosas, no contienen una vinculación directa entre la estructura interna de un acuífero con doble porosidad y los parámetros hidrogeológicos que definen el flujo a través de ella. Esto sólo se ha logrado con geometrías muy simples de la estructura matrizfisura o con realizaciones estocásticas de la red de fisuras que luego requieren un gran esfuerzo computacional al simularlos con modelos hidrodinámicos.

Lo que se presenta en este trabajo de tesis es una metodología sencilla y práctica para lograr mejorar la estimación de los parámetros de un ensayo de bombeo en este tipo de acuíferos confinados naturalmente fracturados, incorporando por primera vez la estructura y la proporción de las familias de fisuras en las ecuaciones de pozo a través de la estimación directa del factor de forma de la función de transferencia, incidiendo principalmente en las etapas tempranas del régimen transitorio del bombeo.

Para verificar su funcionamiento, la metodología desarrollada fue puesta a prueba en algunos casos sintéticos orientados a la interpretación de ensayos de bombeo en régimen variable. Además, se aplicó dicha metodología a la interpretación de las características hidrodinámicas de un acuífero cretácico confinado del Distrito uranífero Cerro Solo, en la provincia de Chubut, Argentina.

A partir de las aplicaciones mencionadas, se discuten los resultados obtenidos por comparación con los ejemplos tomados de la literatura presentando el contraste con soluciones analíticas desarrolladas para casos particulares de acuíferos con doble porosidad y con la re-interpretación de la evolución temporal de las depresiones observadas en el acuífero ensayado.

En las conclusiones se destaca la importancia de la consideración de la doble porosidad en las ecuaciones de flujo, alentando a su aplicación concreta en la práctica de interpretación de los ensayos de bombeo con la metodología presentada en esta tesis y a proseguir su evolución en etapas de desarrollo vinculadas al transporte de solutos en este tipo de acuíferos complejos. 


\section{ABSTRACT}

This dissertation cope with unsteady state flow in a dual porosity aquifer. In our theoretical mainframe, a naturally fractured porous media saturated by a monophasic fluid (i.e. water at normal temperature and pressure conditions) was considered. Therefore, a practical review has been oriented to the following topics: first, geostatistical tools used to simulate aquifer parameters, specially, its double porosity; later, the incorporation of matrix-fracture transfer functions inside the governing system equations for those complex aquifers.

Afterwards, a methodology was implemented with the aim to simulate both singular characteristics mentioned before; indeed, the stochastic representation for the always uncertain fracture network and the estimation of the matrix-fracture transfer function coefficients.

To check its performance, this new methodology was then applied to a series of synthetic cases available in the literature. Also, it was then tested in a real field project. This last test was carried out in a cretaceous confined aquifer within the limits of a future uranium mine district at Cerro Solo, Province of Chubut, Argentina.

Finally, a discussion is included about the comparison of the different outcomes against the registered results examples, the analytical solutions for a simplified double porosity model and the normal pumping test interpretation done for the real case problem.

Conclusions mainly emphasize the importance to include the dual porosity equations when the analysis should consider a suspicious aquifer with this nature and suggest going forward with a solute transport model development by analogous methodology. 


\section{RESUM}

La modelación del flux en mitjans geològics naturalment fracturats depèn principalment de la composició estructural de la relació matriu-fractura i de la forma en què s'aproxime l'intercanvi de fluid entre ambdues fases.

Les dues característiques esmentades (composició estructural de la doble porositat i la funció de transferència de flux) incorporen el major grau d'incertesa en la modelación dels estats transitoris generats per bombament.

Per a poder millorar la interpretació de l'assaig de bombament i amb l'objectiu últim d'obtenir paràmetres característics confiables de l'aqüífer (tals com la conductivitat i el coeficient d'emmagatzematge per a ambdues fases), la metodologia proposada en aquesta tesi aprofita les eines que brinda la geoestadística per a simular realitzacions de camps de fissures que el seu assemble permet al seu torn estimar els paràmetres de forma de la funció de transferència matriu-fissura (FTMF).

En efecte, els mètodes clàssics disponibles per a interpretar resultats d'assajos de bombament en aqüiffers heterogenis, especialment aquells que es presenten naturalment fracturats $i$ amb una complexa relació entre les dues components poroses, no contenen una vinculació directa entre l'estructura interna d'un aqüífer amb doble porositat i els paràmetres hidrogeológicos que defineixen el flux a través d'ella. Açò només s'ha aconseguit amb geometries molt simples de l'estructura matriufissura o amb models estocàstics de la xarxa de fissures que després requereixen un gran esforç computacional en simular-los amb models hidrodinàmics.

El que es presenta en aquest treball de tesi és una metodologia senzilla i pràctica per a aconseguir millorar l'estimació dels paràmetres d'un assaig de bombament en aquest tipus d'aqüífers confinats naturalment fracturats, incorporant per primera vegada l'estructura i la proporció de les famílies de fissures en les equacions de pou a través de l'estimació directa del factor de forma de la funció de transferència, incidint principalment en les etapes primerenques del règim transitori del bombament.

Per a verificar el seu funcionament, la metodologia desenvolupada va ser posada a prova en alguns casos sintètics orientats a la interpretació d'assajos de bombament en règim variable. A més, es va aplicar aquesta metodologia a la interpretació de les característiques hidrodinàmiques d'un aqüífer cretàcic confinat del Districte uranífero Cerro Solament, en la província de Chubut, Argentina.

A partir de les aplicacions esmentades, es discuteixen els resultats obtinguts per comparació amb els exemples presos de la literatura presentant el contrast amb solucions analítiques desenvolupades per a casos particulars d'aqüífers amb doble porositat i amb la re-interpretació de l'evolució temporal de les depressions observades en l'aqüífer assajat.

En les conclusions es destaca la importància de la consideració de la doble porositat en les equacions de flux, encoratjant a la seua aplicació concreta en la pràctica d'interpretació dels assajos de bombament amb la metodologia presentada en aquesta tesi i a prosseguir la seua evolució en etapes de desenvolupament vinculades al transport de soluts en aquest tipus d'aqüífers complexos. 


\section{AgRADECIMIENTOS}

En el recorrido de estudios, investigaciones y de transferencia de conocimientos que se inició en el año 2010 a partir de un trabajo de campo en la Provincia de Chubut (República Argentina), se fueron recibiendo múltiples apoyos que ameritan, todos ellos, un agradecimiento muy especial. Así, en la culminación de esta etapa de tesis doctoral, sentimos que no hay un orden jerárquico de gratitud porque a su turno todos han sido importantes para la construcción de la misma.

Muchas gracias debemos dar a la hospitalidad y el apoyo técnico del Grupo de Hidrogeología de la Universidad Politécnica de Valencia. La dirección de esta obra por parte de Eduardo Cassiraga ha tenido como cualidad notable la voluntad del apoyo perseverante que se necesita para no claudicar en los momentos de zozobra, en especial, cuando las múltiples actividades que desarrollamos no nos permiten avanzar como quisiéramos. Su guía técnica y amistad han hecho todo más sencillo. También hacer extensivo este agradecimiento a Jaime Gómez Hernández por su cordialidad y soporte institucional, a su equipo de colaboradores y becarios.

Al Dr. Mario Hernández de la Facultad de Ciencias Naturales y Museo de la Universidad Nacional de La Plata (Argentina), inspirador del tema vinculado a la doble porosidad en los acuíferos patagónicos. Como siempre se lo hicimos saber: él es el "culpable" de este despropósito.

Al Profesor Florimond De Smedt del Departamento de Hidrología e Ingeniería Hidráulica de la Vrije Universiteit Brussels (VUB, Bélgica) por permitir el uso de la rutina de cálculo $W_{2 p}$ y su programa de optimización para el ajuste de su curva tipo a las observaciones en campo. Estando ya retirado, nos honra el haber podido compartir su clara visión y manejo conceptual de esta temática, repitiendo de esta forma la atmósfera virtuosa y de alta motivación que lograba contagiar en sus clases de postgrado.

A la Gerencia de Exploración de materias primas de la Comisión Nacional de Energía Atómica (CNEA) en la persona de su gerente técnico, Geólogo Roberto Bianchi, por avalar el desarrollo de esta tesis. Y a todo su equipo, en particular, al subgerente de la Regional Patagonia, Geólogo Nilda Marveggio por su constante entusiasmo para dotar a la línea de base ambiental del Distrito Cerro Solo de un conocimiento sistémico y completo para su futuro aprovechamiento.

A la Facultad de Ingeniería de la Universidad Nacional de La Plata, su Departamento de Hidráulica y a la Unidad de Investigación, Desarrollo y Extensión en Hidrología donde se encuentra nuestro lugar de trabajo, por la ayuda institucional recibida para poder realizar nuestras pasantías en Valencia durante el período 2012-2017.

Finalmente, a toda mi familia: a mi esposa Eugenia, a mis hijos Justina, Luisa, Pedro y Clara y a mis padres y hermanas. Porque me acompañaron con entusiasmo en este esfuerzo, porque soportaron estoicamente mis ausencias (aun no estando de viaje, ellos entienden) y porque seguro podremos recordar juntos esta etapa como un ejemplo de superación que valió la pena transitar. 


\section{ÍNDICE DE CONTENIDOS}

RESUMEN

AGRADECIMIENTOS

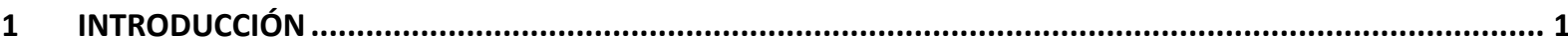

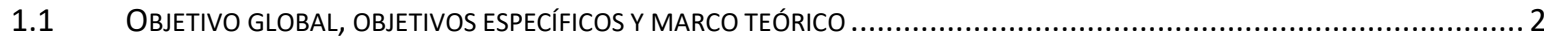

1.2 MODELO CONCEPTUAL, HIPÓTESIS DE TRABAJO Y MÉTODOS DE APROXIMACIÓN.............................................. 3

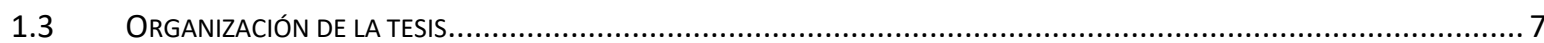

2 ESTADO DEL ARTE DE LA MODELACIÓN DE MEDIOS NATURALMENTE FRACTURADOS ..........................8 8

2.1 MODELOS CONCEPTUALES Y SOLUCIONES ANALÍTICAS CONOCIDAS PARA ACUÍFEROS CON DOBLE POROSIDAD .................8 8

2.1.1 Función de transferencia matriz - fractura (FTMF) en modelos de flujo........................................8

2.1.2 Conductividad hidráulica equivalente - Experiencias físicas en laboratorio................................... 10

2.1.3 Soluciones analíticas y curvas tipo para ensayos de bombeo en medios heterogéneos .................. 12

2.2 HeRRAMIENTAS DE LA GEOESTAdístiCA PARA LA SIMULACIÓN DE MEDIOS NATURALMENTE FRACTURAdOS ..................28

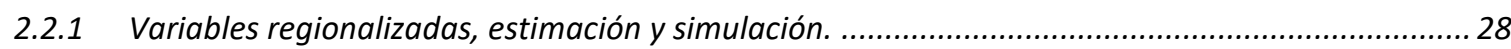

2.2.2 Simulación indicadora secuencial con el programa SISIM ............................................................. 30

2.2.3 Simulación con algoritmos booleanos - El programa ELLIPSIM ................................................... 32

3 MÉTODO COMBINADO PARA EVALUAR ACUÍFEROS CAUTIVOS CON DOBLE POROSIDAD ......................34

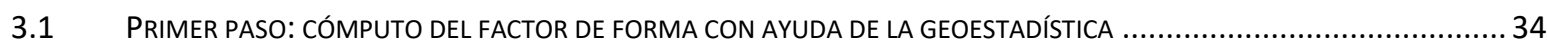

3.1.1 Generación booleana de familias de fracturas con ELLIPSIM .........................................................35

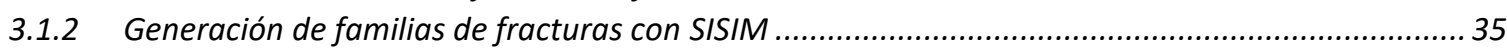

3.1.3 Relación del factor de forma con la proporción de fisuras ............................................................ 36

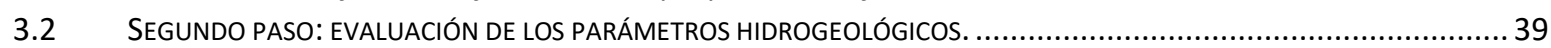

3.2.1 Procedimiento de De Smedt para la interpretación de ensayos de bombeo .................................... 39

3.2.2 Método combinado: secuencia a seguir, opciones y estimación de parámetros. ............................ 41

3.2.3 Ajuste de acuerdo con el tipo de régimen desarrollado en la interface matriz-fisura ..................... 43

4 RESULTADOS DE LA APLICACIÓN DEL MÉTODO PROPUESTO ..............................................................44

4.1 VERIFICACIÓN EN CASOS SINTÉTICOS CON SOLUCIÓN ANALÍTICA CONOCIDA .......................................................44

4.1.1 Modelo cuasi-estacionario de interacción matriz-fisura en acuífero confinado (caso A)................ 44

4.1.2 Acuífero confinado con fracturas planas horizontales (caso B) ...................................................45

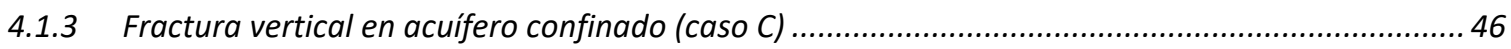

4.2 Aplicación a la formación acuífera del distrito “Cerro Solo”, Chubut, ARgentina ................................46

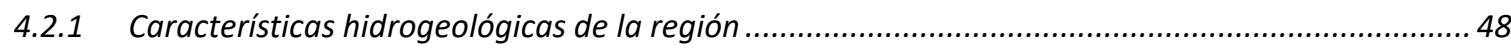

4.2.2 Programa de ensayos de bombeo y resultados obtenidos .........................................................5 50

4.2.3 Aplicación del método combinado propuesto ............................................................................ 51

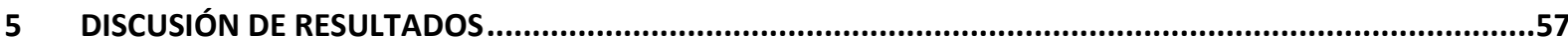

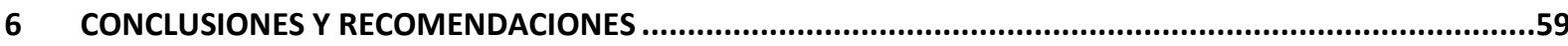

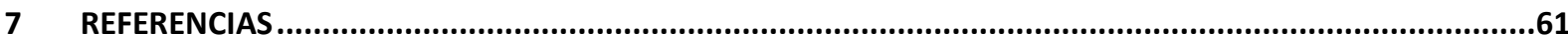

CITADAS EN EL TEXTO

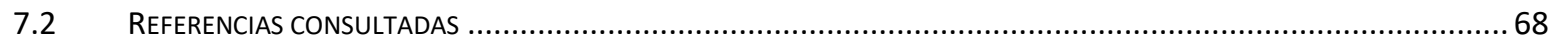

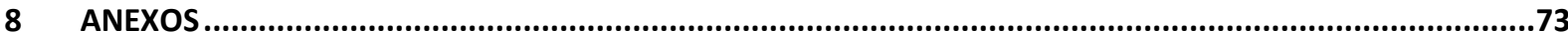

8.1 APLICACIÓN DE LA SOLUCIÓN DE DE SMEDT PARA ACUÍFEROS CON DOBLE POROSIDAD .......................................... 74

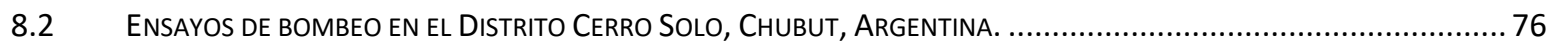

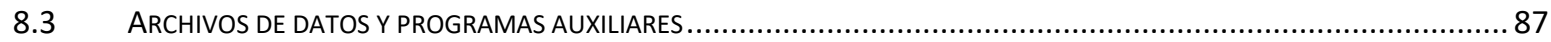




\section{ÍNDICE DE FIGURAS}

Figura 1.1: Representación funcional del sistema acuífero naturalmente fracturado............................3

Figura 2.1: Descenso relativo para el modelo pseudo-estacionario de la FTMF. ................................ 14

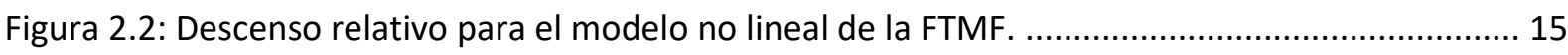

Figura 2.3: Descensos relativos para distintos modelos de la FTMF. ............................................... 16

Figura 2.4: Flujo adimensional para distintos modelos de la FTMF................................................. 17

Figura 2.5: Comparación de distintas aproximaciones a la función de pozo $\mathrm{W}(\mathrm{u})$............................... 20

Figura 2.6: Función de pozo de Hantush W(u,r/B) y su aproximación mediante la ec. (34). .............. 21

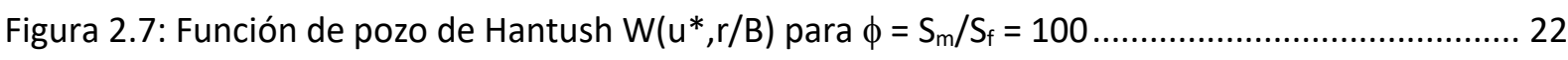

Figura 2.8: Función de pozo de Hantush $\mathrm{H}(\mathrm{u}, \rho)$ para tiempos cortos. ............................................... 23

Figura 2.9: Función de pozo de Barenblatt, $\mathrm{W}_{1}\left(\mathrm{u}_{\mathrm{m}}\right)$ para acuíferos con doble porosidad.................... 24

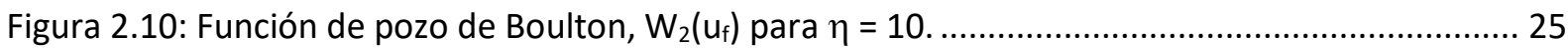

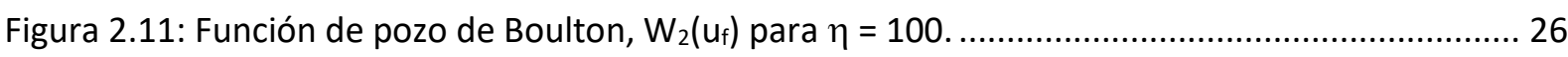

Figura 2.12: Función de pozo para una fractura plana vertical. ...................................................... 27

Figura 2.13: Bloques con fracturas horizontales $(0,0,0)$ y verticales $(90,90,0)$ con SISIM. .................. 31

Figura 2.14: Comparación de bloque generados con distintos modelos de variogramas................... 32

Figura 2.15: Una realización de 3 familias de fracturas generadas con ELLIPSIM (10\% en volumen).. 33

Figura 3.1: Tres familias de fracturas para el cálculo del factor de forma con ELLIPSIM. .................... 35

Figura 3.2: Tres familias de fracturas para el cálculo del factor de forma con SISIM. ......................... 36

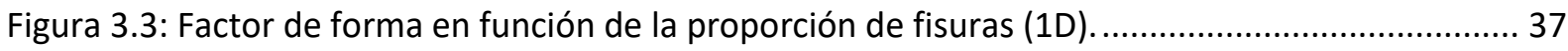

Figura 3.4: Factor de forma en función de la proporción de fisuras (2D) .......................................... 38

Figura 3.5: Factor de forma en función de la proporción de fisuras (3D) .......................................... 38

Figura 3.6: Ejemplo del archivo de datos para el programa de ajuste de De Smedt........................... 39

Figura 3.7: Ejemplo del archivo de resultados del programa de ajuste de De Smedt......................... 40

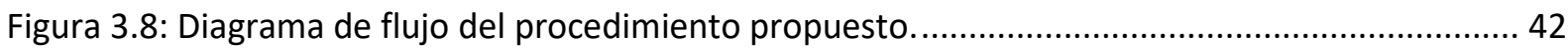

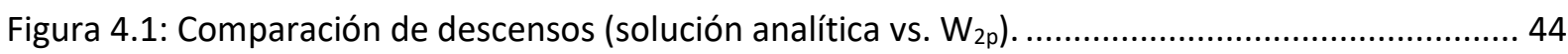

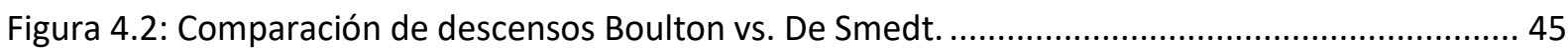

Figura 4.3: Comparación de descensos en los pozos de observación para el caso C. .......................... 46

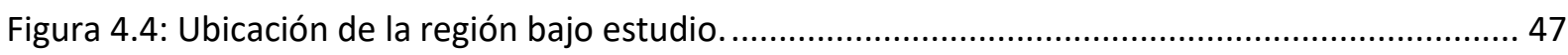

Figura 4.5: Ubicación de los sitios ensayados en la cuenca del arroyo Perdido. ................................. 47

Figura 4.6: Corte geológico AA y BB de la formación (fuente: Hernández et al., 2012). ....................... 48

Figura 4.7: Corte geológico CC y DD de la formación (fuente: Hernández et al., 2012)...................... 49

Figura 4.8: Corte geológico 3D de la formación (fuente: Hernández et al., 2012). ............................. 49

Figura 4.9: Datos del Ensayo de bombeo y ajuste de la W2DP en el sitio Cerro Solo. ......................... 52

Figura 4.10: Datos del Ensayo de bombeo y ajuste de la W2DP en el sitio Puesto Alvear................... 52

Figura 4.11: Datos del Ensayo de bombeo y ajuste de la W2DP en el sitio El Molino............................ 53

Figura 4.12: Datos del Ensayo de bombeo y ajuste de la W2DP en el sitio Arroyo Perdido................. 53

Figura 4.13: Mapa de estructuras geológicas en el Distrito Cerro Solo, Chubut, Argentina. ............... 55

Figura 8.1: Ajustes de la función de pozo $W_{2}$ para el ensayo en el acuífero de Madison. ................... 74

Figura 8.2: Ajustes de la función de pozo $W_{2}$ para el ensayo en el acuífero de Yuca Mountain. ......... 75

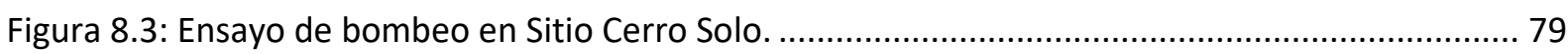

Figura 8.4: Ensayo de bombeo en el Pozo Ensayo 1 (Yacimiento Cerro Solo)....................................... 79

Figura 8.5: Ensayo de bombeo en el Pozo Ensayo 2 (Yacimiento Cerro Solo)..................................... 80

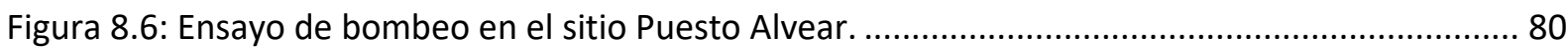

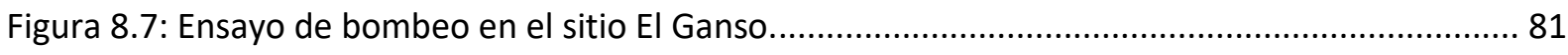

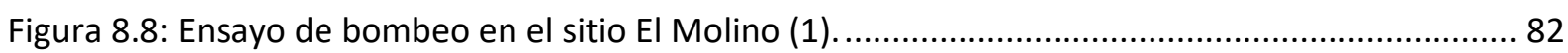


Figura 8.9: Ensayo de bombeo en el sitio El Molino (2).

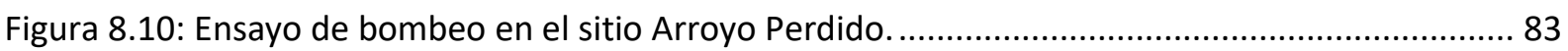

Figura 8.11: Ensayo de bombeo en el sitio El Pique (gran diámetro). .............................................. 84

Figura 8.12: Unidades Hidrogeológicas y ubicación de yacimientos (Hernández et al. 2012). ............ 86

\section{ÍNDICE DE TABLAS}

Tabla 2.1: Cálculo de la permeabilidad dual equivalente para los casos recopilados ........................ 11

Tabla 3.1: Rango de variación del coeficiente de bloque (de Ranjbar y Hassanzadeh,2011).............. 36

Tabla 3.2: Rango del factor de forma para un bloque de $100 \times 100 \times 50$......................................... 37

Tabla 4.1: Estimación de parámetros mediante la solución analítica de De Smedt (2011).................. 51

Tabla 4.2: Parámetros de la Función de transferencia matriz -fisura ................................................... 54

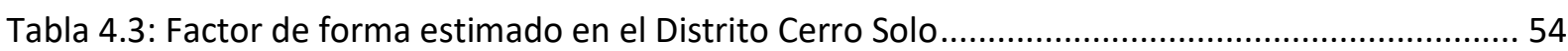

Tabla 4.4: Nuevos parámetros de la Función de transferencia matriz -fisura ....................................... 56

Tabla 5.1: Rango de proporciones de fisuras a partir de los valores de $\alpha$ reportados ........................ 57

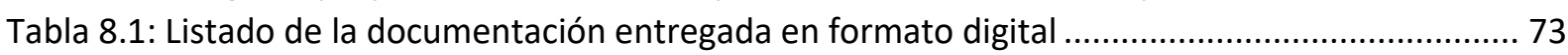

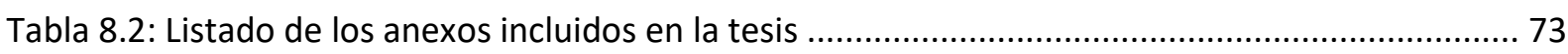

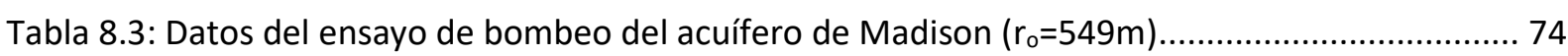

Tabla 8.4: Resultados de los ensayos de bombeo en el yacimiento Cerro Solo .................................... 77

Tabla 8.5: Resultados de los ensayos de bombeo en Puesto Alvear y El Ganso................................... 77

Tabla 8.6: Resultados de los ensayos de bombeo en El Molino y Arroyo Perdido. ............................... 78

Tabla 8.7: Distancias calculadas entre pozos de bombeo y de observación. ....................................... 78

\section{ÍNDICE DE FOTOS}

Foto 4.1: Afloramiento en superficie del Mo. Arroyo del Pajarito....................................................... 48

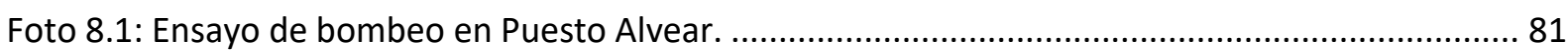

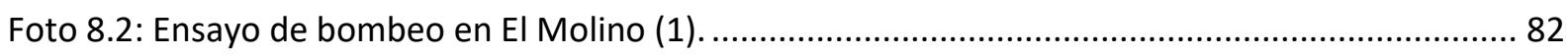

Foto 8.3: Ensayo de bombeo en El Pique (pozo de gran diámetro).................................................... 84 


\section{INTRODUCCIÓN}

Las formaciones geológicas fracturadas son las más frecuentes en la naturaleza (Streltsova, 1978; Singhal y Gupta, 2010; Scesi y Gattinoni, 2009); en efecto, se estima que tres cuartas partes de la superficie global de los continentes está dominada por acuíferos naturalmente fracturados (Dietrich et al., 2005).

Se trata de un sistema hidrológico complejo (De Marsily et al., 2005) integrado por la matriz original, o componente primaria, variando su estructura desde un medio poroso típico con espacios inter-granulares hasta un bloque de roca con baja o nula permeabilidad) y un conjunto de fracturas o fisuras, desde pequeñas diaclasas hasta una falla con extensión regional, y cuya conectividad entre elementos puede generar una extensa red de conducción de flujo (Bear, 1972; Adler et al., 2013).

Ambas componentes de este sistema interactúan con diferente intensidad a través de una interface (entendida como una zona de transición) que debe ser caracterizada estructural y espacialmente (Barenblatt et al., 1960; Warren y Root, 1963; Streltsova, 1978 y 1984; Neuman, 2005) para poder evaluar el flujo de intercambio entre ambas.

En lo que sigue se hará referencia especial a aquellas formaciones geológicas naturalmente fracturadas reconocidas como unidades acuíferas, y con las siguientes características (De Marsily, 1986; Batu, 1998; Schwartz y Zhang, 2003; Kresic, 2007; Lemonnier y Bourbiaux, 2010)):

- $\quad$ capacidad para almacenar y transmitir agua en todos sus componentes (matriz y fisuras);

- saturación plena de los espacios interconectados de ambas componentes;

- comportamiento elástico de su estructura ante cambios importantes de la presión;

- estabilidad química (sin disolución) de sus materiales constituyentes.

La selección de este entorno de trabajo está directamente relacionada con las aplicaciones que se contemplan en los próximos capítulos de esta tesis. Constituye así un núcleo central de análisis que no reduce su generalidad dado que son las características más frecuentes de estas formaciones acuíferas con doble porosidad (Pinder y Celia, 2006; Sen, 2009; Sahimi, 2011; Delgado, 2012).

No obstante, se debe destacar que existe una gran cantidad de estudios dedicados a medios con contenidos de humedad inferiores a la porosidad total (Nitao y Buscheck, 1991; Wu et al., 2004; Hunt y Ewing, 2009), con múltiples fases fluidas (de Swaan, 1976; Douglas y Arbogast, 1990), con deformaciones permanentes de la estructura porosa (Bai y Elsworth, 2000) debido a procesos de consolidación/compactación por drenaje (subsidencia) y/o con procesos de disolución de la roca madre (cavidades progresivas); dichos estudios son los más desarrollados en los campos de actuación de la ingeniería de reservorios (Bogdanov et al., 2003) y de las actividades mineras, entre ellas, la de producción de hidrocarburos (Royer et al., 1996; Bourbiaux et al., 1998; Yuan y Harrison, 2006; Bogatkov y Babadagli, 2010; Presho et al., 2011), en aplicaciones típicas de la geomecánica y en una amplia rama de la Hidrogeología que investiga a las formaciones kársticas (Bakalowicz, 2005; Goldscheider y Drew, 2007), respectivamente.

En el desarrollo de esta tesis se incluyeron avances metodológicos eminentemente prácticos que pueden relacionarse a las distintas ramas mencionadas que se desprenden del entorno de análisis elegido, dando lugar así a futuras líneas de investigación que se comentan en el capítulo final. 


\subsection{OBJetIVO GLOBAL, OBJeTIVOS ESPECÍFICOS Y MARCO TEÓRICO}

El objetivo global del presente trabajo fue desarrollar una metodología que en la práctica de los ensayos de bombeo permita mejorar la estimación de los parámetros hidrogeológicos de un acuífero naturalmente fracturado.

Para ello, se plantearon objetivos específicos que permitieran llevar adelante:

- un estudio detallado, gradual y progresivo del conocimiento prexistente acerca de la forma de modelar los acuíferos naturalmente fracturados con una visión sistémica del proceso de interacción matriz-fractura,

- una evaluación de las metodologías actualmente disponibles para la generación estocástica de una red de fracturas con las herramientas que brinda la Geoestadística,

- una propuesta metodológica que permita mejorar la interpretación de los ensayos de bombeo en acuíferos naturalmente fracturados.

Comenzando de esta manera a definir el marco teórico del presente trabajo, existen múltiples combinaciones de interconexión del sistema matriz-fractura que se pueden considerar de acuerdo con el estado de integración de las mismas (Duguid y Lee, 1977; Ahmed et al., 2008; Romanazzi y Cassiraga, $2014 a, b)$. Pero antes que eso, resulta necesario comentar brevemente las características de cada una de las componentes del sistema por separado:

- La matriz original. En primer término, si su origen es de carácter sedimentario entonces será válida la teoría clásica de flujo en medio poroso saturado (Ley de Darcy, 1856). Hay entonces una capacidad finita de almacenar y de transmitir (recibir/ceder) agua (Scesi y Gattinoni, 2009; Singhal y Gupta, 2010). Por el contrario, si la matriz estuviera constituida por una roca sólida o un aglomerado consolidado con alta cementación de tal manera que su continuidad en el espacio sea solo interrumpida por las superficies (planas o con curvatura) de las fracturas existentes, no existiría en este caso participación de la matriz en procesos subterráneos activos de acumulación o de transferencia de agua (Zimmerman y Bodvarsson, 1994; Krásny, 2003). La gama de variantes entre estos dos casos extremos queda definida por la eficiencia de llevar adelante el almacenamiento y/o cesión de agua desde la unidad matriz (Barenblatt et al, 1960; Warren y Rooy, 1963; Choi et al., 1997; Neuman, 2005; Nonner, 2010, Stadler et al., 2012), pudiendo agruparse estos estados intermedios bajo las denominaciones de medio con doble porosidad (cuando la matriz cumple el rol de unidad de almacenamiento y el flujo principal se desarrolla a través de la red de fracturas) o de medio con doble permeabilidad (matriz y red de fracturas participando ambas activamente del flujo general pero en forma diferenciada en función de sus respectivas conductividades hidráulicas);

- El subsistema de fracturas (o fisuras). La propiedad que surge como más relevante es la conectividad entre las distintas familias presentes en una formación geológica (Cushman, 1990; Neuman, 2005; Bogdanov, 2007). En cada familia de fracturas/fisuras (Koike et al., 2012) se pueden identificar sus características geométricas y proyección estereográfica (orientación y desarrollo en el espacio), otros atributos significativos como la separación, curvatura y rugosidad relativa de las paredes de la fractura, la intensidad de la disgregación, presencia de material de relleno o de zonas de contacto entre las mismas (Klimczak et al., 2010) y, por último, las intersecciones entre las diferentes familias de fracturas. Todas estas 
características determinan el régimen (laminar o turbulento) y el tipo de resistencia al escurrimiento (pared lisa o rugosa) que se puede manifestar dentro de la red de fracturas, en una analogía muy cercana a los diagramas de resistencia de los escurrimientos a presión en tuberías (De Marsily, 1986; Scesi y Gattinoni, 2007).

\subsection{MODELO CONCEPTUAL, HIPÓTESIS DE TRABAJO Y MÉTODOS DE APROXIMACIÓN}

Los modelos conceptuales que emergen de estas primeras consideraciones del complejo matriz-fractura son variados, cada uno destacando las propiedades que se consideran dominantes en alguna de sus componentes. Una visión global de todas esas variantes puede sintetizarse a partir de la consideración del siguiente esquema funcional (Romanazzi y Cassiraga, 2014b):

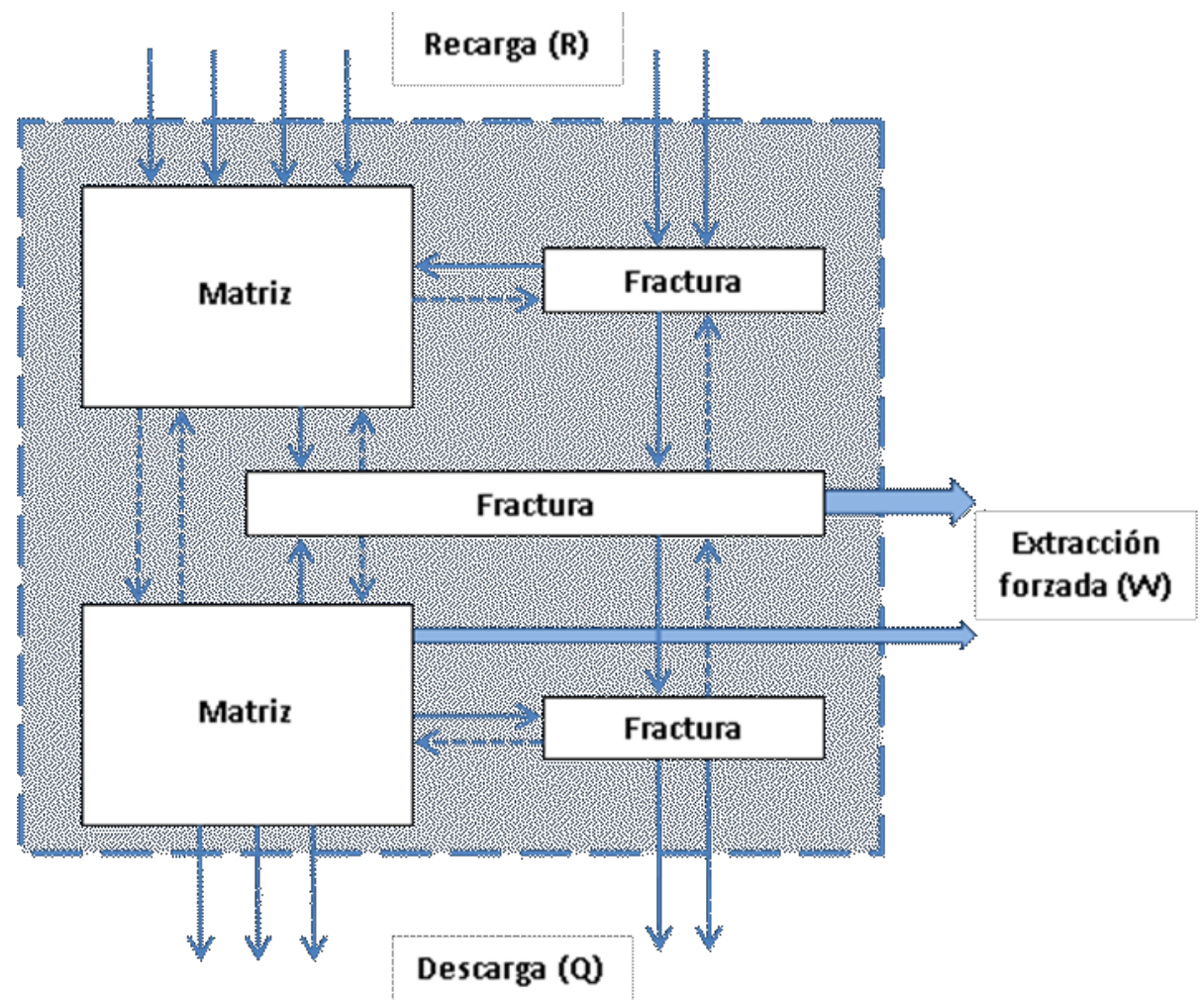

Figura 1.1: Representación funcional del sistema acuífero naturalmente fracturado.

Existe posibilidad de recarga (natural o artificial) o descarga (libre o por bombeo) del sistema tanto hacia/desde la componente matricial como directamente hacia/desde las unidades de fractura. Estas condiciones de borde externas (son las líneas de corriente que atraviesan los límites externos del sistema representado en la Figura 1.1) determinan, a partir del flujo neto resultante $(Q+W-R$, en la misma figura), que se puedan experimentar distintos estados de evolución temporal posibles (estacionario o transitorio) de los niveles de energía del sistema. Entre las condiciones de borde internas se destaca la interconectividad de las unidades de fractura, lo que puede dar lugar, como ya se mencionara, a una red propia y muy desarrollada de flujo. Los bloques de matriz también pueden considerarse conectados directamente entre sí, pero con enlaces por lo general más débiles comparados con el anterior. Ejemplo de esto son las formaciones yuxtapuestas horizontalmente con diferente permeabilidad y que terminan aislando (confinando) a las capas inferiores. La importancia de la transferencia de flujo entre estos bloques o capas queda así supeditada a la diferencia de energía piezométrica entre ellos. 
Cuando se consideran entonces diferencias de conductividad/permeabilidad entre componentes (matriz y fisuras) y entre las mismas componentes (acuitardos intercalados entre formaciones acuíferas o combinaciones de fisuras con distinto espesor y/o con relleno intersticial) se clasifica al sistema no sólo como de doble porosidad sino como de doble permeabilidad. También existen trabajos que hacen referencia a combinaciones superiores de estas propiedades ( $\mathrm{n}$-porosidad, $\mathrm{n}$-permeabilidad).

La Figura 1.1 destaca el intercambio bidireccional de masa fluida entre los componentes principales del sistema (matriz y fractura), resultando su comportamiento temporal en estado transitorio la principal motivación para el desarrollo de esta tesis.

En consecuencia, para este modelo conceptual se adoptan aquí las siguientes hipótesis:

I. Propiedades físicas del fluido: monofásico, en este caso agua en estado líquido, con una densidad y viscosidad aproximadamente constante en el rango de presiones y de temperaturas de trabajo (Civan, 2011). Los casos especiales (presiones positivas o negativas importantes, disolución de sales que modifiquen la masa específica del agua, transporte de sustancias/solutos, mezcla con gases o líquidos de mayor viscosidad) no se consideran en el presente análisis.

II. Escala de trabajo: es factible elegir para cada componente una escala representativa o adecuada a la anisotropía del medio (Freeze, 1975), sea ésta una componente primaria del sistema (bloque matriz) o una familia determinada del conjunto de fracturas (Gerke, 1996). Se desarrolla aquí una escala local porque el aporte metodológico de esta tesis se centra en las ecuaciones de pozo aplicadas en medios porosos naturalmente fracturados, siendo válida su aplicación en la zona de influencia del bombeo central.

III. Almacenamiento interno: se considera factible la alimentación del bloque matriz a partir de una unidad de fractura. Asimismo, la matriz cede agua a la red de fracturas si el gradiente de presiones así lo favorece. De cualquier modo, el almacenamiento principal del sistema se encuentra alojado en la componente matricial y no en la red de fracturas.

IV. Transferencia interna: la hipótesis anterior implica que la interface matriz - fractura es permeable. Surge inmediatamente la necesidad de definir esa componente de flujo interno haciendo explicita su participación como fuentes/sumideros en las ecuaciones de continuidad que se postulen por separado para cada una de las componentes del sistema (Gerke y Van Genuchten, 1993a,b).

V. Continuidad del medio: en arreglo a la hipótesis precedente, la componente "matriz" del sistema es un dominio esencialmente discontinuo, franqueado o limitado por la componente "fractura" con mayores chances de aproximarse a la concepción de un medio continuo si se verifica la conectividad entre sus propios elementos. Cuando esta red de fracturas logra una conectividad eficiente, el flujo se desarrolla principalmente por esa vía.

VI. Flujo externo al sistema: es el resultado neto de masa circulante por unidad de tiempo (salidas menos entradas) que atraviesa los contornos superficiales del sistema, tal como se plantea tradicionalmente en la deducción de la ecuación de continuidad en la mecánica de fluidos (principio de conservación). El bombeo de la fuente subterránea es considerado aquí como el principal perturbador del estado natural del sistema, dando origen a los estados transitorios de mayor interés en el ámbito definido para esta tesis. 
VII. Variación del Almacenamiento: la variación del almacenamiento dentro del volumen de control del sistema debe computarse teniendo en cuenta las variaciones individuales en cada una de las componentes (matriz, fractura) en el mismo período de tiempo.

VIII. Estados transitorios provocados por bombeo: en condiciones naturales el gradiente piezométrico (o freático para acuíferos no confinados) determina la dirección y el sentido del escurrimiento subterráneo cuasi-estacionario, tanto para la escala local (flujo 2D y 3D) como para la regional (flujo horizontal dominante). Las situaciones producidas por bombeo son consideradas como exportación (extracción) de agua del sistema, donde la red de fracturas aporta el volumen inicial a una tasa muy elevada en correspondencia con su mayor trasmisividad (T). La prolongación en el tiempo del bombeo disminuye rápidamente la reserva de agua en la fractura y se complementa con el abastecimiento más lento de los bloques de la matriz original de la formación rocosa (Kazemi, 1969; Stadler et al., 2012). Se produce así una transición en la evolución temporal de los descensos piezométricos, en forma muy similar a las interpretaciones que se hacen de los valores experimentales de un ensayo de campo para el caso de una recarga diferida. Si en las proximidades del pozo de bombeo el volumen de fisura se agota, entonces se produce un corte (o un escalón) en los caudales de bombeo que sólo puede renovarse si se dejan recuperar los niveles y, por ende, se permite incorporar el volumen que le transfiere la matriz a la red de fisuras.

Este amplio abanico de intensidades de conducción del agua en el sistema planteado (matriz - fractura) deriva en uno de los principales núcleos de incertidumbre acerca de su comportamiento hidrogeológico (Hyndman y Lewis, 2007): no siempre se cuenta con información de campo que permita identificar con precisión cuál es el caso dominante, dado que varios de ellos pueden coexistir en un mismo acuífero. Además, se debe agregar a este análisis, la incertidumbre asociada a los parámetros que caracterizan un medio permeable anisótropo, entre ellos, el tensor conductividad hidráulica (K) en cada localización. El manejo más satisfactorio a esta última fuente de incertidumbre se ha logrado con las técnicas geoestadísticas de simulación estocástica.

En función del grado de síntesis que se pretenda aplicar al esquema global mostrado en la Figura 1.1, se pueden mencionar (Cushman, 1990; Singhal y Gupta. 2010) los siguientes modelos de aproximación:

1) Modelo poroso equivalente (MPE): en este caso todo el sistema acuífero es considerado como un medio continuo donde sólo se manifiestan diferencias significativas entre los valores de la conductividad hidráulica $(K)$ de los distintos bloques de matriz y fracturas. Cuando se carga el sistema con un gradiente hidráulico, cada celda o bloque conductivo transfiere internamente el caudal específico de acuerdo con las direcciones dominantes que marca el tensor $\mathrm{K}$ promediado localmente. En la escala regional y para régimen permanente o estacionario, se suele estimar una conductividad equivalente (Ke), brindado así resultados aceptables para el cómputo del balance hídrico en esa escala. También puede ser aceptada la analogía siguiente (Douglas y Arbogast, 1990; Streltsova, 1978): si la matriz posee bloques de baja o nula permeabilidad y la red de fracturas es lo suficientemente densa, su estructura interna puede ser asimilada a un medio macro-granular (o macro-poroso) con verificación global y aproximada de la ley de Darcy.

2) Modelo de red de fracturas discretas (RFD): el medio permeable se idealiza como una red de nodos y conectividades de muy variada extensión y orientación espacial. Los esfuerzos por caracterizar la intrincada red de fracturas van desde vuelos aerofotogramétricos, 
relevamientos en campo, ensayos geofísicos y hasta imágenes derivadas de tomografías subterráneas (Bourbiaux et al., 1998). Además de la orientación, se relevan y describen estadísticamente la apertura, el relleno y la frecuencia o densidad de fracturación (proximidad y cantidad de fracturas en una unidad de referencia lineal o volumétrica), la persistencia de la discontinuidad (porcentaje de la extensión alcanzada con respecto a un patrón lineal o superficial), rugosidad de sus paredes y reducción de la fracturación con la profundidad (Scesi y Gattinoni, 2009). La representación de todas estas medidas en el modelo puede ser determinística o estocástica, prevaleciendo esta última como método más efectivo (Vert et al., 1998; Zang, 2002). En la mayoría de los casos, las observaciones de las discontinuidades sólo conforman una muestra pequeña de un universo mucho más complejo y por ese motivo se intenta una generación probabilística de la red de fracturas (Rubin, 2003). Los objetos seleccionados para la representación de las fracturas (discos, planos, elipses) se distribuyen en el espacio (centro geométrico, separación y orientación) de acuerdo con distribuciones exponenciales, log-normales o del tipo Poisson o, en forma alternativa, mediante simulación indicadora secuencial sin suponer a priori una distribución probabilística (método de simulación no paramétrico que nos brinda la geoestadística). En un paso posterior, la asignación de conductividades a esos objetos también puede realizarse en forma determinística asimilando el flujo en la fractura como un escurrimiento entre dos placas planas paralelas. Así, se trabaja con una expresión analítica similar a la Ley de Darcy para medios porosos pero que ahora depende del cubo de la separación entre planos. De ese tipo de relaciones surgen entonces las altas conductividades a considerar. Estos modelos han sido utilizados con éxito en acuíferos de baja permeabilidad genética o de base, pero donde la red de fracturas termina representando una vía real y preferencial de flujo, propiedad adquirida con el tiempo a través de diferentes procesos geológicos.

3) Modelo de doble porosidad (MDP): en este tipo de modelos lo que más se intenta aproximar a la realidad del acuífero es la interacción matriz-fractura. Esto se logra incorporando una ecuación que especifica la transferencia de masa fluida entre esas dos componentes en función del diferencial de presiones reinante en la zona de contacto. Además, esta aproximación se distingue de otras porque las conductividades de la matriz y de la fractura son modeladas en forma conjunta, pero en ecuaciones separadas (Sahimi, 2011; Adler et al., 2013). En estas circunstancias, se suele denominar a este modelo como de doble porosidad y de doble permeabilidad, destacando que la heterogeneidad del medio proviene de la consideración conjunta de ambas variables. Para plantear entonces este modelo se debe considerar, aparte de la ecuación de continuidad (balance de masa), un sistema de ecuaciones dinámicas que se construye con tres expresiones analíticas acopladas: Ley de Darcy para la matriz, Ley cúbica para la fractura y una ecuación de transferencia entre ambas. Este sistema de ecuaciones puede plantearse sobre la base de asimilar las variables y los coeficientes (parámetros) que integran las mismas como determinísticos o como aleatorios. Las aplicaciones a casos sintéticos con hipótesis simplificadas producen resultados muy coherentes.

4) Modelo estocástico continuo (MEC): como su nombre lo indica, estos modelos trabajan con el concepto de variable aleatoria regionalizada y hacen uso de las herramientas que ofrece la geoestadística. Se analizan los campos espaciales de la matriz y de cada una de las familias de fracturas, atribuyendo un variograma a cada componente y estimando sus valores por krigeado para todo el dominio modelado, pero en bloques separados (Langevin, 2002). Estos bloques luego se acoplan remplazando en cada celda la conductividad que le indica un índice de referencia (matriz, fractura tipo 1, fractura tipo 2, etc.). Desde el punto de vista de la dinámica del flujo, si bien los factores geométricos y litológicos (estratificación) se encuentran considerados, este procedimiento se comporta de aquí en más como un modelo 
de medio continuo tradicional, pero en versión estocástica (Lu y Connell, 2011). Dependiendo de la densidad y calidad de la información que se le suministre, se cuenta con una metodología más potente para evaluar la variabilidad y la incertidumbre espacial asociada: trabajar con algún método de simulación estocástica para obtener múltiples realizaciones, por ejemplo, de la matriz y de distintas familias de fracturas con una geometría especificada y derivar así múltiples campos de conductividades $\mathrm{K}$. Adicionalmente, se cuenta con la posibilidad de incorporar información secundaria para mejorar la estimación y la simulación de los parámetros que caracterizan el acuífero. La reproducción de niveles piezométricos con esta metodología (Gómez-Hernandez, Sahuquillo y Capilla, 1997; Hendricks-Franssen et al., 1998) ha dado buenos resultados con un esfuerzo menor comparado con la dificultad que implica la caracterización de una red de fracturas (Modelo RFD) o con la de incorporar una ecuación de transferencia entre ambas componentes del medio (MDP).

En base a todo lo considerado hasta aquí y como ya se había adelantado, surge como principal motivación para el desarrollo de esta tesis las posibilidades que puede llegar a ofrecer una combinación de las ventajas y complementación de los modelos MDP y MEC. En efecto, el modelo MDP es elegido por su capacidad para reproducir con mayor detalle los estados transitorios cuando comienzan a disminuir los aportes de agua desde de la red de fracturas y se inicia el abastecimiento desde la matriz; y el modelo MEC por su versatilidad para simular campos aceptables de porosidad y/o permeabilidad (condicionado a las observaciones disponibles) del medio complejo así considerado.

\subsection{ORGANIZACIÓN DE LA TESIS}

Para materializar este planteo se estableció como prioritario iniciar el trabajo de investigación con un relevamiento del estado del arte en la materia (capítulo 2), para luego pasar a desarrollar un procedimiento metodológico para mejorar la estimación de los coeficientes que integran la función de transferencia matriz-fisura (FTMF) que, combinado con soluciones analíticas disponibles, permite obtener un conocimiento ampliado de los parámetros hidrogeológicos de estos acuíferos complejos de porosidad dual (capítulo 3).

Para verificar el método desarrollado se lo pone a prueba en casos sintéticos seleccionados y se lo aplica también a una experiencia real de campo en un acuífero confinado cercano a la localidad de Paso de Indios, provincia de Chubut, Argentina (capítulo 4). Seguidamente se discuten resultados (capítulo 5) y se desarrollan conclusiones y recomendaciones para posibles líneas futuras de investigación (capítulo 6).

En la recopilación de antecedentes se ha podido lograr una numerosa colección de artículos, libros y trabajos inéditos que se anexan a esta tesis en formato digital (se los incluye en un DVD aparte).

Como un grupo de esas publicaciones no han sido relacionadas directamente con los contenidos de la presente tesis, se las agrupa como referencia consultada (capítulo 7) para permitir su posible acceso ulterior.

Finalmente, en los anexos (capítulo 8) se han agregado desarrollos y transcripciones de informes inéditos que complementan lo citado en el cuerpo principal de la tesis. También, se incluyen datos y programas auxiliares para que futuros usuarios puedan aplicar libremente el método desarrollado en este trabajo. 


\section{ESTADO DEL ARTE DE LA MODELACIÓN DE MEDIOS NATURALMENTE}

FRACTURADOS

En este apartado se incluye un resumen de la recopilación efectuada sobre la modelación de acuíferos naturalmente fracturados, dividiendo la presentación en dos partes: primero, una sección dedicada a los distintos esquemas conceptuales y soluciones analíticas conocidas para considerar la porosidad secundaria en modelos de acuíferos y una breve referencia a experiencias físicas llevadas a cabo en laboratorio para la imbibición de una matriz columnar con un grado de fisuración controlado y que orientan a la estimación de las magnitudes de sus propiedades hidrogeológicas (conductividad y almacenamiento); en segundo lugar, una descripción de las posibilidades que ofrece la Geoestadística para la simulación estocástica de familias de fracturas, factor principal de la heterogeneidad espacial que presentan este tipo de medios.

\subsection{MOdelos CONCEPTUALES Y SOLUCIONES ANALÍTICAS CONOCIDAS PARA ACUífEROS CON DOBLE POROSIDAD}

\subsubsection{Función de transferencia matriz - fractura (FTMF) en modelos de flujo}

El flujo entre dos medios con tan disímil constitución (un medio poroso matricial con flujo en régimen laminar vs. una red de fisuras con rápida respuesta a los cambios de presión) debe incluir la discontinuidad espacial como una condición de borde interna y evaluar el caudal a través de la interfase con una función de transferencia específica (Custodio y Llamas, 1996).

La función de transferencia del flujo fue presentada hace ya más de medio siglo atrás por Barenblatt, Zheltov y Kochina (1960) y por Warren y Root (1963) como el atributo más destacable a incorporar en este tipo complejo de medio subterráneo.

Diferentes propuestas han sido dadas para la expresión analítica de esta función de transferencia, denominada de aquí en más con la sigla FTMF (Función de Transferencia Matriz Fractura), la mayoría de ellas basadas en una dependencia directa del gradiente de presiones en la frontera de la discontinuidad (Dietrich et al, 2005; Neuman, 2005). Para llevar adelante este paso es necesario recurrir primero a una identificación georeferenciada de dichos límites internos y, en cada uno de sus elementos donde se evalúa el flujo, proceder luego a aplicar la FTMF con la diferencia actual de presiones en ambas caras de la frontera.

La estrategia seguida por diferentes autores (Hantush, 1964 y 1966; Boulton y Streltsova, 1978; Streltsova, 1978, 1984; De Smedt, 2011) para computar la diferencia de presiones responde al grado de aproximación elegido para llevar adelante la discretización de las componentes de este sistema heterogéneo. Típicamente se ha considerado que la presión matricial es un escalar representativo de todo el bloque poroso aislado (o delimitado) por la red de fisuras (Barenblatt et al., 1960; Streltsova, 1976a), mientras que esta última es la encargada de expresar los gradientes que movilizan o modulan la intensidad del flujo por unidad de área. Se suele referenciar a este caso como modelo lineal o pseudo-estacionario de transferencia.

En los modelos de doble porosidad más simples, los bloques de la componente matricial juegan el rol de unidad de almacenamiento debido a su alta porosidad (y relativa baja permeabilidad) mientras que la componente de la red de fracturas cumple con el desempeño de conducción preferencial del flujo (Boulton y Streltsova, 1978a yb). 
Otra de las cualidades esenciales de este modelo tiene que ver con la posibilidad cierta de transferencia de fluido dentro y entre bloques de matriz aún con la baja permeabilidad relativa apuntada. A largo plazo (luego de, por ejemplo, un transitorio provocado por bombeo) se puede dar un equilibro interno en el cual se contribuye simultáneamente al transporte de flujo desde el almacenamiento matricial y desde la red de fracturas (De Swaan, 1976).

De este modo, se puede sostener como una buena hipótesis que ese será el estado a que tienda el sistema a largo plazo, pudiendo considerarse como un medio continuo equivalente (MCE) cuya conductividad hidráulica (K) y su almacenamiento específico $\left(\mathrm{S}_{\mathrm{e}}\right)$, son los promedios pesados de la contribución relativa de los bloques de matriz y red de fracturas (Renard y De Marsily, 1997):

$$
\begin{aligned}
& \bar{K}=w_{f} K_{f}+w_{m} K_{m} \\
& \overline{S_{e}}=w_{f} S_{e f}+w_{m} S_{e m}
\end{aligned}
$$

donde los subíndices $f$ (fractura) y $m$ (matriz) indican la componente del sistema dual así planteado; $w_{i}[-]$ es el volumen ocupado por cada componente referido al volumen total de la formación $\left(w_{f}+w_{m}=1\right) ; K_{i}$ es la conductividad hidráulica de cada medio [ $\left[T^{-1}\right] ;$ y $S_{e}$, como ya se adelantó, es el almacenamiento específico $\left[\mathrm{L}^{-1}\right]$.

El sistema acoplado de ecuaciones correspondiente a este tipo de modelo (en el cual rige en forma aproximada la ley de Darcy para ambas componentes), se puede escribir como sigue (Gerke y Van Genuchten, 1993a,b):

$$
\begin{aligned}
& \nabla\left[K_{f}(x) \nabla h_{f}(x, t)\right]=S_{e f} \frac{\partial h_{f}(x, t)}{\partial t}-f_{T}(x, t) \quad \text { (fractura) } \\
& \nabla\left[K_{m}(x) \nabla h_{m}(x, t)\right]=S_{e m} \frac{\partial h_{m}(x, t)}{\partial t}+f_{T}(x, t) \quad \text { (matriz) }
\end{aligned}
$$

donde $x$ y $t$ son las variables independientes espacio y tiempo, respectivamente; $f_{T}(x, t)$ es la función de transferencia matriz-fractura $\left[\mathrm{T}^{-1}\right]$ que representa al flujo unitario entre ambas fases por unidad de volumen; y $\mathrm{h}$ es la energía piezométrica [L].

Cabe reiterar entonces que (3) y (4) surgen como combinación de la ecuación de continuidad con la ecuación de Darcy (1856) y se ha prescindido de acciones externas como los bombeos o la inyección forzada de agua (tomados en la literatura básica como fuentes y/o sumideros del campo de flujo) reflejando así el comportamiento natural del sistema (Douglas \& Arbogast, 1990; Pinder \& Celia, 2006).

La función de transferencia $f_{T}(x, t)$ tiene que ver entonces con una cesión/aporte de agua interno (condición de borde interna) que no necesariamente debe darse bajo depresiones provocados por bombeo. La misma ha sido expresada analíticamente bajo diversas formas (Boulton y Streltsova, 1977a,b; De Smedt, 2011), pero la versión que puede presentarse como denominador común de todas ellas es la siguiente:

$$
f_{T}(x, t)=\alpha K_{i}\left[h_{f}-h_{m}\right]
$$

donde $\alpha$ es un factor de forma $\left[\mathrm{L}^{-2}\right]$ cuya expresión depende de la manera en que se conceptualice la geometría del sistema matriz-fractura, entre otros, como un juego simple de fracturas paralelas o doble familia ortogonal de fracturas, elementos matriciales cúbicos o esféricos (Lim y Aziz, 1995; Hassanzadeh et al., 2009; Lai y Pao, 2013; ; y $\mathrm{K}_{\mathrm{i}}\left[\mathrm{LT}^{-1}\right]$ es la conductividad hidráulica intrínseca de la interfase matriz-fractura, en muchas ocasiones asimilada a la conductividad de la matriz $\left(\mathrm{K}_{\mathrm{m}}\right)$ por simplicidad. 
Al factor de forma $(\alpha)$, se le adjudica también un efecto de revestimiento o relleno de la fractura por mineralización de la roca adyacente (Moench, 1984; Chang y Yeh, 2011), análogo al que se produce en las inmediaciones de una perforación donde el material alterado puede actuar como amplificador o reductor de la trasmisividad (skin factor) en función de la naturaleza de su constitución.

En la medida que este factor resulte importante, el retardo de la respuesta del medio poroso matricial se hace evidente, acercándose así al modelo cuasi-estacionario de flujo de Barenblatt (1960). En ese sentido y como señalan Streltsova (1984) y Neuman (2005), es necesario también conocer la evolución del transitorio hidráulico desde el momento mismo en que se manifiesta la alta difusividad que proporciona la red de fracturas como respuesta a un cambio de presiones y la acción retardada de la matriz aportando el volumen necesario para abastecer las vías preferenciales de flujo que las primeras conforman. Así, la forma en que se modela la expresión (5) y la caracterización (inicio y forma de evolución) de la acción retardada de la matriz, han permitido inferir propiedades básicas de estos acuíferos complejos.

Finalmente, el factor de forma $(\alpha)$ fue también muy estudiado no sólo en función de las posibles características geométricas del medio fracturado (Hassanzadeh et al., 2006, 2009; Ranjbar et al. 2011; Lai y Pao, 2013), sino que se ha adaptado también a la versión no estacionaria de la función de transferencia cuando no resulta despreciable la variación de presiones dentro del medio poroso primario (matriz).

\subsubsection{Conductividad hidráulica equivalente - Experiencias físicas en laboratorio.}

Para la evaluación de las propiedades hidráulicas en medios naturalmente fracturados, se han identificado en la bibliografía dos líneas de desarrollo con diferentes formas de abordaje: una formulación determinística (Zimmerman et al., 1993; Gerke y Van Genuchten, 1993a,b) y, otra más reciente, de tipo estocástica (Zhang, 2002; Arora et al., 2012).

En la aproximación determinística, Zimmerman et al. $(1993,1996)$ simularon numéricamente la inyección de agua a carga constante en un medio con doble porosidad comparando los distintos regímenes de declinación del gasto con el modelo de infiltración desarrollado por Nitao y Buscheck (1991).

Contemporáneamente, Gerke y Van Genuchten (1993) experimentan con un modelo de caudal variable (en el tiempo) e ingresante a una familia de fracturas paralelas rodeadas de un medio poroso que las contiene.

Si bien estos ejemplos pueden ser clasificados como de flujo no saturado y corresponden más a experiencias de percolación en una columna de un medio heterogéneo, el sentido del frente húmedo avanzando desde la fractura y embebiendo la matriz es un caso posible (recarga) del modelo conceptual aquí contemplado, y por lo tanto, aportan a la descripción de la transferencia matriz-fractura como también puede darse en el sentido inverso de circulación, encontrando analogías en los transitorios alcanzados por bombeo en acuíferos libres (Streltsova, 1973; Boulton y Streltsova, 1978), o semiconfinados (Zimmerman y Bodvarsson, 1994).

En la aproximación estocástica de estas ecuaciones, la estructura del sistema es similar a lo descripto en el apartado anterior con la diferencia de que ahora los parámetros $w(x), K(x)$ y $\alpha(x)$, integrantes de las ecuaciones de flujo y de transferencia inter-porosa, son ahora variables aleatorias espacialmente distribuidas (Nowak et al., 2008; Freeze, 1975). 
De esta forma, la resolución del sistema de ecuaciones para el planteo del problema directo no sólo proporciona un valor medio estimado de la energía piezométrica en cada nodo, sino que la incertidumbre asociada a la estimación de esa variable se puede expresar, por ejemplo, mediante el valor de su varianza en esa coordenada (Zhang y Sun, 2000; Zhang, 2002).

En la misma línea, el trabajo desarrollado por Arora et al. (2012) permitió contrastar un modelo estocástico con la medición del campo de presiones y flujo en una columna de suelo limo-arenoso (la matriz) más un set de macro-poros en la mitad de la base circular del dispositivo (las fisuras) materializados con tubos capilares de acero de $1 \mathrm{~mm}$ de diámetro. La densidad de los macro-poros se varió para poder cuantificar así la influencia de esta componente secundaria y analizar la sensibilidad de los parámetros estimados para el medio poroso, en especial, la conductividad hidráulica de la interfase matriz-fractura $\left(\mathrm{K}_{\mathrm{i}}\right)$.

Al tender naturalmente a un equilibrio en las presiones reinantes en la interfase, la estimación de una conductividad hidráulica compuesta o equivalente para el sistema dual puede lograrse de acuerdo con la expresión analítica (1). Por otro lado, cuando se cuenta con indicios de la existencia de una doble porosidad en el medio acuífero, la práctica habitual de la estimación de su permeabilidad consiste en "penalizar" (reducir en al menos un orden de magnitud) las cifras de la conductividad "temprana" que proviene principalmente de la red de fracturas.

En la Tabla 2.1 se consignan las conductividades recopiladas en este trabajo y se calcula (última columna) un valor compuesto o equivalente.

Tabla 2.1: Cálculo de la permeabilidad dual equivalente para los casos recopilados

\begin{tabular}{ccccc} 
Caso de estudio & $\begin{array}{c}\mathrm{K}_{\mathrm{f}} \\
{[\mathrm{m} / \mathrm{s}]}\end{array}$ & $\begin{array}{c}\mathrm{K}_{\mathrm{m}} \\
{[\mathrm{m} / \mathrm{s}]}\end{array}$ & $\begin{array}{c}\mathrm{W}_{\mathrm{f}} \\
{[-]}\end{array}$ & $\begin{array}{c}\mathrm{K} \\
{[\mathrm{m} / \mathrm{s}]}\end{array}$ \\
\hline Nitao y Buscheck (1991) & $8,2 \times 10^{-3}$ & $1,9 \times 10^{-11}$ & $2,0 \times 10^{-2}$ & $1,6 \times 10^{-4}$ \\
Zimmerman y Bodvarson. (1993) & $9,8 \times 10^{-9}$ & $9,8 \times 10^{-12}$ & $9,6 \times 10^{-4}$ & $1,9 \times 10^{-11}$ \\
Gerke y Van Genuchten (1993) & $2,3 \times 10^{-4}$ & $1,2 \times 10^{-9}$ & $5,0 \times 10^{-2}$ & $1,2 \times 10^{-5}$ \\
Zhang (2002) & $3,7 \times 10^{-1}$ & $5,0 \times 10^{-2}$ & $1,0 \times 10^{-2}$ & $5,3 \times 10^{-2}$ \\
& & & $1,7 \times 10^{-5}$ & $3,6 \times 10^{-7}$ \\
Arora, Mohanty y McGuire (2012) & $2,3 \times 10^{-5}$ & $3,6 \times 10^{-7}$ & $5,2 \times 10^{-5}$ & $3,6 \times 10^{-7}$ \\
& & & $3,3 \times 10^{-4}$ & $3,6 \times 10^{-7}$ \\
\hline
\end{tabular}

Como surge de la misma, se cumple aproximadamente la metodología expeditiva para la estimación global a largo plazo de la conductividad. En efecto, la conductividad hidráulica equivalente $(K)$ resulta un orden de magnitud inferior que la conductividad de la red de fisuras $\left(\mathrm{K}_{\mathrm{f}}\right)$, siendo este último el valor inicial que se adopta para la conductividad a corto plazo.

En lo referido al almacenamiento, también existe una combinación de ambas componentes (ec. 2 ) que directamente se suman ( $S_{\text {equivalente }}=S_{m}+S_{f}$ ) en un período avanzado de tiempo para abastecer en forma conjunta a la extracción por bombeo. Todos los modelos que se desarrollan a continuación coinciden en esta característica (Hantush, 1964; Douglas y Arbogast, 1990; De Smedt, 2011). 


\subsubsection{Soluciones analíticas y curvas tipo para ensayos de bombeo en medios heterogéneos}

Para poder disponer de un patrón de comparación con los resultados obtenidos en ensayos de bombeo en acuíferos naturalmente fracturados, se identificaron en la bibliografía casos típicos de estudio en los cuales se haya podido llegar a una solución analítica por integración de las ecuaciones de flujo.

Las "ecuaciones de pozo" que se seleccionaron en base al modelo conceptual que se toma como referencia en este trabajo (Hantush, 1964; Streltsova, 1978 y 1984; Huyakorn et al., 1983; Custodio y Llamas, 1996), fueron los siguientes:

A. Acuífero confinado con doble porosidad (Modelo de Barenblatt).

B. Acuífero confinado con fracturas horizontales (Modelo de Boulton).

C. Acuífero confinado con pozo penetrante en fractura vertical (Modelo de Gringarten).

El ejemplo $A$ responde originalmente al modelo conceptual de acuífero confinado con doble porosidad (Barenblatt et. al.,1960). En los casos siguientes (B y C), la definición de las características de la red de fracturas resulta imprescindible (aunque particularmente sencillo de hacer) para aplicar las soluciones dadas por Boulton (1963) y Gringarten (1974), respectivamente.

\subsubsection{Modelo general para acuíferos porosos naturalmente fracturados.}

Redefiniendo las expresiones (3), (4) y (5) para un escurrimiento radial concurrente a un pozo de bombeo (Streltsova, 1978; De Smedt, 2011) en un medio poroso naturalmente fracturado, se tiene:

$$
\begin{gathered}
T\left(\frac{\partial^{2} d_{f}}{\partial r^{2}}+\frac{1}{r} \frac{\partial d_{f}}{\partial r}\right)=S_{f} \frac{\partial d_{f}}{\partial t}-F_{T} \\
0=S_{m} \frac{\partial d_{m}}{\partial t}+F_{T} \quad \text { (fisura) } \\
\text { con } F_{T}=b f_{T}=\alpha b K_{i}\left(d_{f}-d_{m}\right)=C\left(d_{f}-d_{m}\right)
\end{gathered}
$$

donde:

- $\quad T\left[L^{2} T^{-1}\right]=K_{f} b$, es la trasmisividad del medio fracturado (en este caso asimilable a la capacidad de trasmitir principalmente el agua a través de su red de fracturas),

- $d_{f}=h_{o}-h_{f}$ y $d_{m}=h_{o}-h_{m}$ (con $h_{o}$ como altura piezométrica inicial) son los descensos piezométricos [L] en las fracturas y en la unidad matriz, respectivamente;

- b [L] el espesor saturado del acuífero;

- $\quad r$ [L] es la distancia radial al pozo de bombeo;

- $\quad t[T]$ es el tiempo desde el inicio del bombeo;

- $\quad S=S_{e} b[-]$, coeficiente de almacenamiento (con subíndice $m$ ó f según corresponda); 
- $\quad \mathrm{F}_{\mathrm{T}}\left[\mathrm{LT}^{-1}\right]$, es ahora la FTMF definida para todo el espesor saturado b;

- $\quad C=\alpha K_{i} b\left[T^{-1}\right]$, es el coeficiente de transferencia de la FTMF.

- $\quad \alpha\left[\mathrm{L}^{-2}\right]$, que como se mencionó antes es un coeficiente de forma que computa el cuadrado de la superficie total de las interfases matriz-fisura por unidad de volumen.

Las hipótesis básicas de este modelo de flujo son:

a) que la conductividad del medio se debe fundamentalmente a la red de fracturas (con lo cual $\mathrm{T}_{\mathrm{f}}=\mathrm{T}$ en la ec. 6); $\mathrm{y}$

b) que la variación del flujo que atraviesa la matriz es despreciable y lo único que prospera es el flujo de intercambio con las fisuras (lo cual anula completamente el primer miembro de la ec. 7).

Las condiciones iniciales para imponer son que los descensos sean nulos en ambas fases para $\mathrm{t}=0$, lo cual implica que:

$$
d_{f}(r, 0)=d_{m}(r, 0)=0 \text {, con } h_{o}=h_{m}=h_{f} .
$$

Por otro lado, las condiciones de contorno están vinculadas a valores límites del radio r:

$$
\lim _{r \rightarrow 0}\left(r \frac{\partial d_{f}}{\partial r}\right)=-\frac{Q}{2 \pi T} ; \quad d_{f}(r \rightarrow \infty, t)=0
$$

o en palabras que en las proximidades del pozo de bombeo se cumple la Ley de Darcy con un caudal de extracción $\mathrm{Q}\left[\mathrm{L}^{3} \mathrm{~T}^{-1}\right]$; mientras que, para una posición suficientemente alejada del pozo central, el descenso es prácticamente nulo.

Pero antes de integrar la ecuación de flujo, resulta de interés saber cómo se comporta la FTMF en la zona de contacto, o dicho de otro modo, cómo influye el término que da origen a la función de transferencia $\mathrm{F}_{\mathrm{T}}$ al haber despreciado el Laplaciano de (7).

Para esto último (Streltsova, 1977 y 1978), se debe considerar el gradiente de la línea piezométrica en la dirección normal ( $n$ ) a la zona de transición matriz-fractura recordando que:

$$
F_{T}=b f_{T}=S_{m} \frac{\partial d_{m}}{\partial t}=-K_{i} \frac{\partial d_{i}}{\partial n}
$$

donde $d_{i}$ es el descenso en la zona de interfase entre matriz y fractura de espesor $b^{\prime}$ en la dirección n. Como antes, el coeficiente $\mathrm{K}_{\mathrm{i}}\left[\mathrm{LT}^{-1}\right]$ es la conductividad propia de esa transición.

La integración de (9) en todo el ancho b':

$$
\int_{0}^{b^{\prime}} S_{m} \frac{\partial d_{m}}{\partial t} d n=-\int_{0}^{b^{\prime}} K_{i} \frac{\partial d_{i}}{\partial n} d n
$$

teniendo en cuenta que para $i=0$ es $d_{i}=d_{f}$ y para $i=b^{\prime}$ es $d_{i}=d_{m}$, resulta:

$$
b^{\prime} S_{m} \frac{\partial d_{m}}{\partial t}=K_{i}\left(d_{f}-d_{m}\right) \quad \text { ó } \quad \frac{\partial d_{m}}{\partial t}=\beta_{i}\left(d_{f}-d_{m}\right)
$$

donde $\beta_{i}=K_{i} /\left(b^{\prime} S_{m}\right)\left[T^{-1}\right]$. Al integrar (11) con respecto al tiempo se obtiene: 


$$
d^{*}=\frac{d_{m}}{d_{f}}=1-e^{-\beta_{i} t}=1-e^{-t^{*}}
$$

con $\mathrm{t}^{*}=\beta_{\mathrm{i}} \mathrm{t}[-]$, tiempo adimensional.

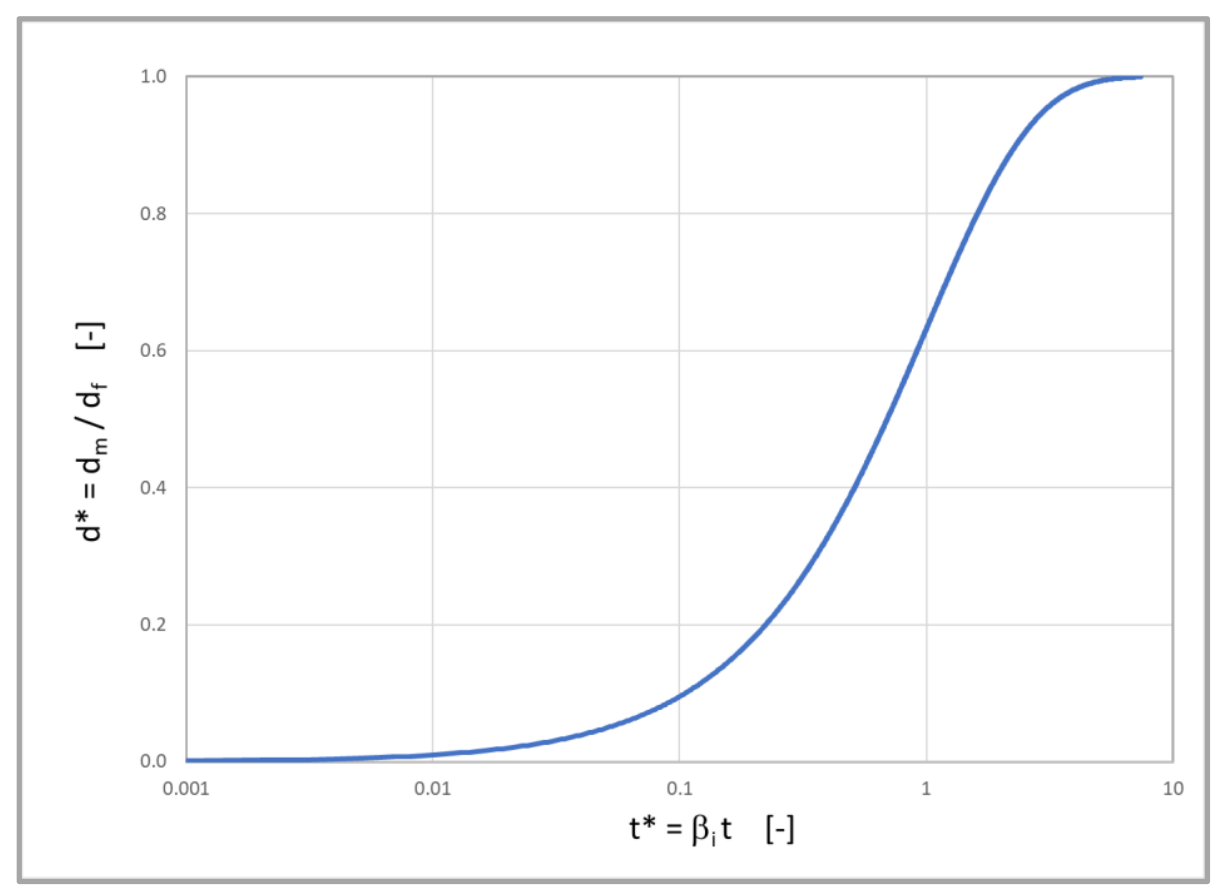

Figura 2.1: Descenso relativo para el modelo pseudo-estacionario de la FTMF.

En la Fig. 2.1 se muestra que la diferencia de presiones entre ambos extremos de la interfase tiende a igualarse con el tiempo. En este modelo pseudo-estacionario de la FTMF, la interfase es concebida como un bloque matricial de espesor activo b' donde la caída lineal de presión se produce en el momento en que se deprime el medio a través de la red de fracturas que, por su alta conductividad, reacciona primero.

En modelos no lineales (Streltsova, 1984; Neuman 2005) el gradiente de presiones se propaga dentro de la interfase, razón por la cual, en un gráfico como el anterior se visualizaría toda una familia de curvas no sólo dependiente de $t^{*}$ sino de la distancia relativa $i^{*}=\mathrm{i} / \mathrm{b}^{\prime}[-]$ de la interface matriz-fractura.

En efecto, si se considera ahora que el gradiente piezométrico involucra al bloque de la matriz, entonces el estado transitorio viene dado por:

$$
F_{T}=b f_{T}=S_{m} \frac{\partial d_{m}}{\partial t}=-K_{i} \frac{\partial^{2} d_{i}}{\partial n^{2}}
$$

que resulta similar a la expresión (9) excepto que ahora en el medio de menor permeabilidad relativa (en este caso, la matriz) el término difusivo es no lineal. La integración de (13) a lo largo de la dirección normal a la zona de interfase y bajo las siguientes condiciones de contorno:

$$
\text { para } \mathrm{i} \rightarrow 0 \Rightarrow \mathrm{d}_{\mathrm{i}} \rightarrow \mathrm{d}_{\mathrm{f}} \quad ; \quad \text { para } \mathrm{i} \rightarrow \mathrm{b}^{\prime} \Rightarrow \frac{\partial \mathrm{d}_{\mathrm{i}}}{\partial \mathrm{n}} \rightarrow 0
$$

conduce a la siguiente solución aproximada (Streltsova, 1978, 1984): 


$$
d^{*}=\frac{d_{m}}{d_{f}} \cong 1-\frac{4}{\pi} \sum_{j=1,3,5, \ldots}^{\infty} \frac{1}{j} \operatorname{sen}\left(\frac{j \pi}{2} i^{*}\right) e^{-\left(\frac{j \pi}{2}\right)^{2} t^{*}}
$$

donde $i^{*}=\mathrm{i} / \mathrm{b}^{\prime}[-]$ es una distancia relativa (en dirección normal a la transición fisura-matriz) que ahora involucra al bloque matriz desde $i=0$ (en contacto con la fisura) hasta $i=b^{\prime}$ (en muchos casos idealizado con la mitad de la dimensión promedio del bloque matricial).

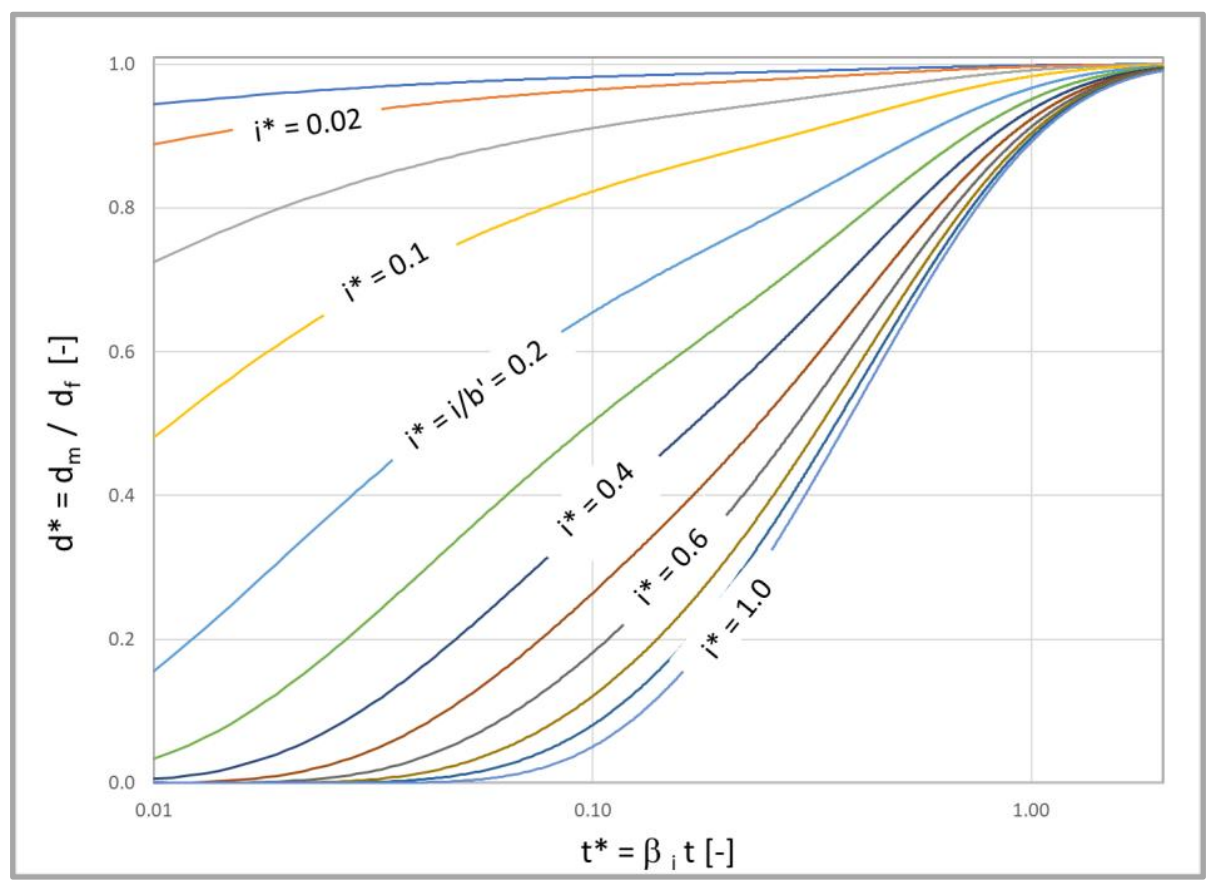

Figura 2.2: Descenso relativo para el modelo no lineal de la FTMF.

La Figura 2.2 fue construida a partir de los primeros 100 términos de la serie (15), con lo cual el error por truncamiento de la misma resulta despreciable. Lo que se aprecia en la figura es la coherencia con los límites extremos del bloque, en especial cuando las familias de $i^{*}=i / b^{\prime} \rightarrow 0$ se comprueba que $d^{*}=d_{m} / d_{f}=1$ para todo $t^{*}$.

Pero como ya se ha señalado, lo interesante aquí es poder comparar ambos modelos para poder evaluar su comportamiento en el tiempo. Como se explicó antes, existe un diferimiento (o retardo) del sistema (interpretado como un modelo lineal o pseudo-estacionario) para reaccionar ante un cambio de presión en la red de fisuras. Para hacer válida la comparación es necesario calcular un promedio de lo que sucede a lo ancho de toda la zona de transición ( $\left.b^{\prime}\right)$.

Dicho promedio (Streltsova, 1984) responde al siguiente desarrollo en serie:

$$
d^{*}=\frac{d_{m}}{d_{f}} \cong 1-\frac{8}{\pi^{2}} \sum_{j=1,3,5, \ldots}^{\infty} \frac{1}{j^{2}} e^{-\left(\frac{j \pi}{2}\right)^{2} t^{*}}
$$

En cuanto a la expresión (16) puede afirmarse entonces que ahora el descenso relativo no depende más de la coordenada espacial (es, como se citó antes, un promedio integrado a lo largo de la longitud representativa de la zona de interface o dimensión del bloque matricial) pero temporalmente muestra una anticipación para alcanzar valores de equilibrio entre ambos descensos, según se aprecia en la siguiente figura: 


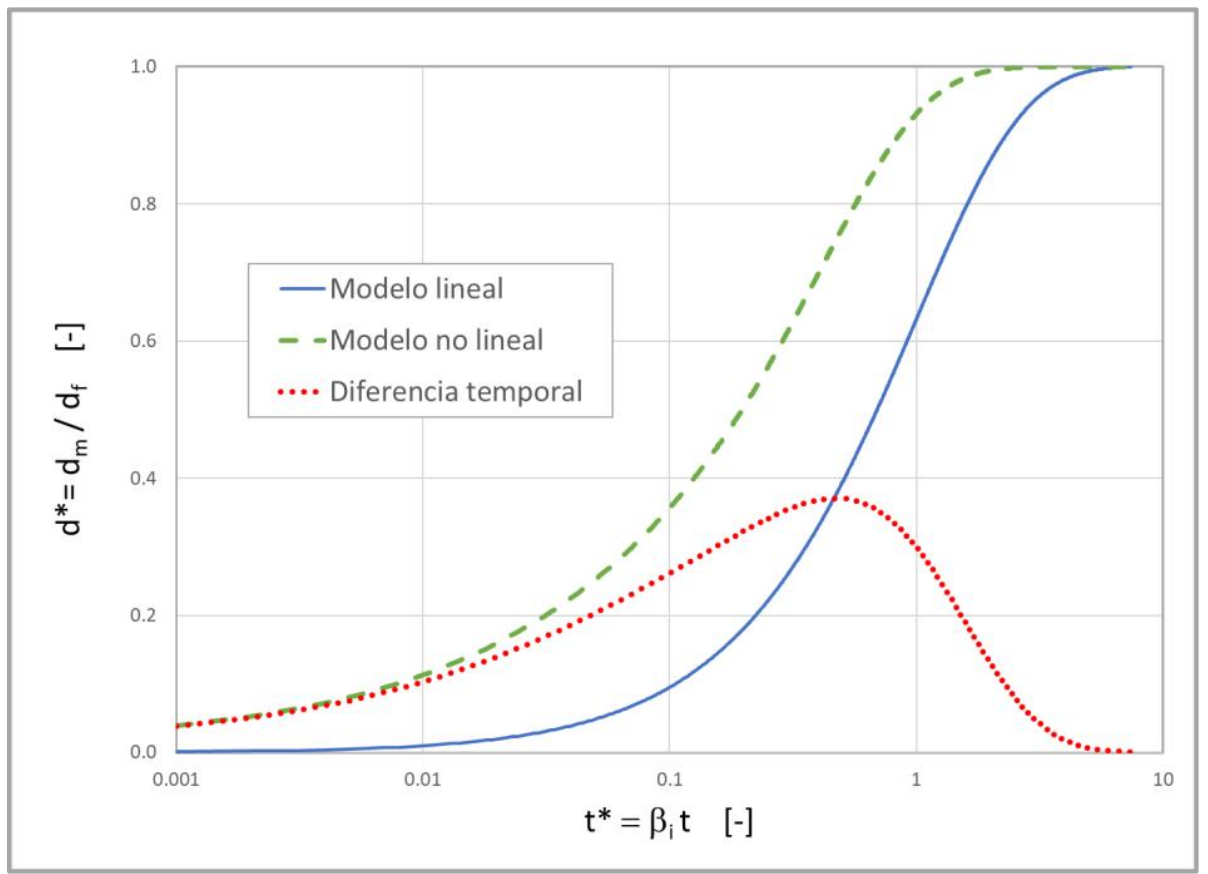

Figura 2.3: Descensos relativos para distintos modelos de la FTMF.

La curva de trazos de la Figura 2.3 se obtuvo como promedio del modelo no lineal (Fig. 2.2) al integrarlo desde $\mathrm{i}=0$ hasta $\mathrm{i}=\mathrm{b}^{\prime}$.

La diferencia entre los dos modelos presenta el mayor apartamiento para $t^{*} \cong 0,475$. En ese instante el descenso en la matriz es el $74 \%$ respecto del descenso en la fractura para el modelo no lineal, mientras que para el modelo semi-estacionario esa cifra es del $37 \%$ (prácticamente duplica su valor).

En términos del caudal específico de la FTMF ambos modelos se pueden expresar analíticamente como sigue:

$$
\begin{array}{ll}
F_{T}=S_{m} \beta_{i}\left(d_{f}-d_{m}\right)=\frac{K_{i} d_{f}}{b^{\prime}} e^{-t^{*}} & \text { (Modelo lineal) } \\
F_{T}=\frac{2 K_{i} d_{f}}{b^{\prime}} \sum_{j=1,3,5, \ldots}^{\infty} e^{-\left(\frac{j \pi}{2}\right)^{2} t^{*}} & \text { (Modelo no lineal) }
\end{array}
$$
la FTMF:

Las ecuaciones (17) y (18) se pueden modificar para obtener una versión adimensional de

$$
F_{T}^{*}=\frac{F_{T} b^{\prime}}{K_{i} d_{f}}
$$

y poder comparar ambos modelos a lo largo de su evolución temporal.

En la figura siguiente se han representado ambas versiones de la FTMF donde se destaca que las mismas sólo se asemejan cuando han transcurrido largos períodos de tiempo: 


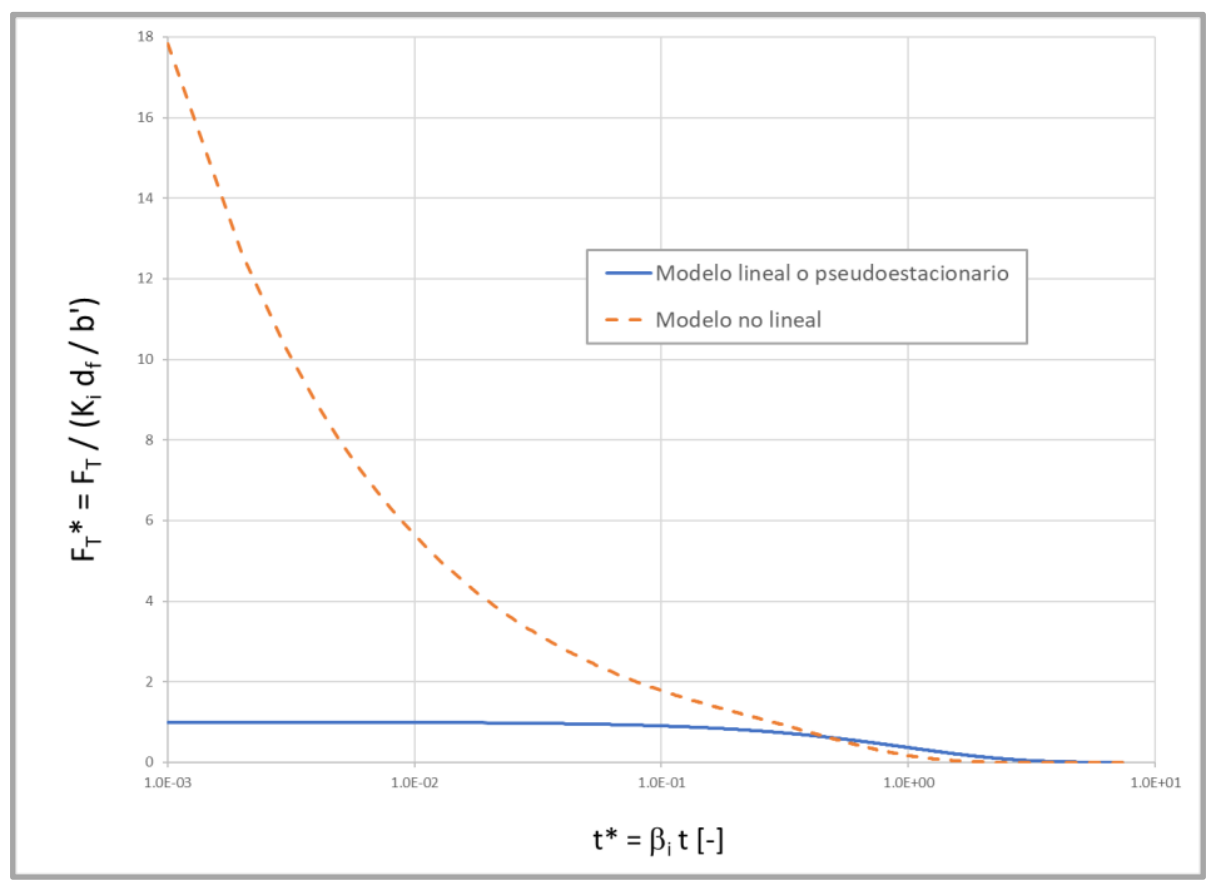

Figura 2.4: Flujo adimensional para distintos modelos de la FTMF.

En conclusión, el modelo no lineal de la FTMF reproduce una cesión de agua hacia el sistema de fisuras a una tasa más alta y, en consecuencia, se logra equilibrar más rápido la diferencia de presiones entre ambas fases. En ese sentido, a igualdad de factores resistivos $\left(\mathrm{K}_{\mathrm{i}}\right)$ y amortiguadores de la transferencia de flujo $\left(\mathrm{S}_{\mathrm{m}}\right)$, cuando existen tamaños grandes de bloques matriciales (mayor b') aportando a la red de fracturas resulta más probable que el comportamiento se asemeje al modelo pseudo-estacionario, con baja tasa de intercambio y con una lenta recuperación del equilibrio entre las presiones de ambas componentes del medio geológico.

\subsubsection{Modelo base de acuífero confinado en medio homogéneo e isótropo, con caudal de extracción constante y penetración total de su espesor (modelo de Theis).}

Siguiendo con la integración de las ecuaciones de flujo, entre las diversas técnicas analíticas y numéricas que se usan habitualmente, se encuentra la aplicación de la Transformada de Laplace a la expresión original en derivadas parciales.

En efecto, si se considera en primera aproximación un acuífero confinado sin fracturación en su matriz porosa (modelo elemental de medio homogéneo e isótropo) y se cumplen que el escurrimiento radial a un pozo es el resultado de un bombeo a caudal constante, se llega a una ecuación de tipo parabólico, conocida también como ecuación de conducción de calor (por analogía con ese fenómeno físico) de la siguiente forma:

$$
\left(\frac{\partial^{2} d}{\partial r^{2}}+\frac{1}{r} \frac{\partial d}{\partial r}\right)=\frac{S}{T} \frac{\partial d}{\partial t}
$$

Todas las variables incluidas en (20) han sido definidas con anterioridad, pero en este caso se vinculan a un medio poroso tradicional con las siguientes condiciones:

$$
\lim _{r \rightarrow 0}\left(r \frac{\partial d}{\partial r}\right)=-\frac{Q}{2 \pi T} ; \quad d(r \rightarrow \infty, t)=0 ; d(r, 0)=0
$$


Se puede demostrar que la solución de la ecuación (20) es de la forma (Theis,1935; Sternberg, 1969; Custodio y Llamas, 1996):

$$
d=\frac{Q}{4 \pi T} W(u) \quad \text { con } \quad u=\frac{S r^{2}}{4 T t}[-]
$$

donde la función de pozo $W(u)$ (o función de Theis) es una integral exponencial cuya expansión en serie se puede resumir de la siguiente forma:

$$
W(u)=-E_{i}(-u)=\int_{u}^{\infty} \frac{e^{-x}}{x} d x=-\gamma-\ln (u)-\sum_{m=1}^{\infty}(-1)^{m} \frac{u^{m}}{m \cdot m !}
$$

donde $\mathrm{E}_{\mathrm{i}}(\mathrm{u})$ es la integral exponencial de primer orden (con argumento $\mathbf{u}$ perteneciente a los números reales) y donde $\gamma=0,5722156 \ldots$ es la constante de Euler. Esta solución (22) de la ecuación de flujo se obtiene por medio de sustitución y separación de variables para dar lugar a una de las versiones más conocidas y utilizadas para interpretar el transitorio hidráulico de un acuífero cautivo, con bombeo a caudal constante desde un pozo central y con penetración completa en todo su espesor. Constituye un estándar y se suele usar como referencia para resolver casos más complejos, en especial, aquellos donde ya no es válida la hipótesis de homogeneidad del medio permeable.

Como se mencionó antes en este mismo apartado, la ecuación (2) puede ser resuelta también por aplicación del método de la Transformada de Laplace, cuyo operador principal es:

$$
\mathcal{L}(f(t))=F(p)=\int_{0}^{\infty} f(t) e^{-p t} d t
$$

La aplicación de este operador a la (22) da como resultado la siguiente versión de la ecuación diferencial ordinaria de Bessel:

$$
\left(\frac{d^{2} D}{d r^{2}}+\frac{1}{r} \frac{d D}{d r}\right)=\frac{S}{T} p D
$$

bajo las condiciones equivalentes en el campo de la transformada de Laplace, a saber:

$$
\lim _{r \rightarrow 0}\left(r \frac{d D}{d r}\right)=-\frac{Q}{2 \pi p T} ; \quad D(r \rightarrow \infty, p)=0
$$

donde $p$ es el parámetro de Laplace y $D(r, p)$ es la transformada de la función de descenso $d(r, t)$. La solución conocida de (24) es una combinación lineal de las funciones modificadas de Bessel de primero $\left(I_{0}\right)$ y de segundo orden $\left(K_{\circ}\right)$, ambas de primera especie. Su expresión analítica es:

$$
D(r, p)=C_{1} I_{0}\left(r \sqrt{\frac{T}{s} p}\right)+C_{2} K_{o}\left(r \sqrt{\frac{T}{s} p}\right)
$$

con $\mathrm{C} 1$ y C2, constantes a determinar en función de las condiciones de borde mencionadas.

Siguiendo el paso indicado, las constantes se integran a la expresión (24) para dar la solución particular en el campo de la transformación aplicada (Sternberg, 1969): 


$$
D(r, p)=\frac{Q \sqrt{\frac{s}{T}} K_{o}\left(r \sqrt{\frac{T}{s} p}\right)}{2 \pi r_{w} p^{3 / 2} K_{1}\left(r_{w} \sqrt{\frac{T}{s} p}\right)}
$$

donde $r_{w}[L]$ es el radio del pozo de bombeo y $K_{i}$ son las funciones modificadas de Bessel de segundo especie y orden i-ésimo. Las funciones $\mathrm{K}_{\circ}$ y $\mathrm{K}_{1}$ están tabuladas y son fácilmente accesibles desde los entornos de programación o desde planillas de cálculo.

Para volver a la variable original corresponde anti-transformar el resultado que aporta la expresión (23) con alguna fórmula conocida. Entre otras, se encuentra la inversión de la Transformada de Laplace propuesta por Schapery (Najurieta, 1980; Sternberg, 1969) que resulta ser una formulación sencilla de aplicar:

$$
d(r, t)=\left|\mathcal{L}^{-1}\{D(r, p)\}=p D\right|_{p=\frac{1}{2 t}}
$$

y reemplazando (28) en (27) queda:

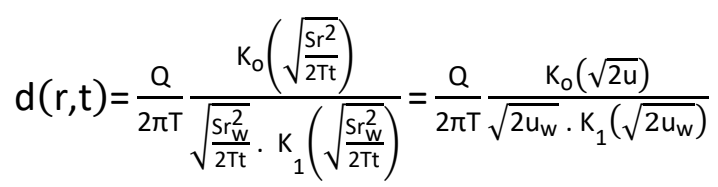

donde $u_{w}$ es el argumento de la función de Theis posicionado en $r=r_{w}$. Comparando con la expresión original de la función de pozo, se tiene que:

$$
w(u) \cong \frac{2 k_{o}(\sqrt{2 u})}{\sqrt{2 u_{w}} \cdot k_{1}\left(\sqrt{2 u_{w}}\right)}
$$

y aprovechando una propiedad de la función $\mathrm{K}_{1}$, si se cumple que su argumento es menor que 0,01 entonces todo el denominador de la (30) tiende a la unidad, para dar lugar así a la siguiente aproximación:

$$
w(u) \cong 2 K_{o}(\sqrt{2 u})
$$

Otra fórmula propuesta para la $\mathrm{W}(\mathrm{u})$ es la siguiente expresión (atribuida a Sternberg en Custodio y Llamas, 1996):

$$
w(u) \cong \frac{2 K_{o}(2 u)}{\sqrt{u \cdot k_{1}(2 u)}}
$$

Finalmente, para su aplicación en la presente tesis se desarrolló una solución que, partiendo de (29) y (32), mejoró el ajuste a la función de Theis (22):

$$
w(u) \cong \frac{k_{o}(\alpha u)}{\sqrt{\alpha u \cdot k_{1}(\alpha u)}}
$$

con una constante $\alpha=1,950445 \cong 1,95$. Esta última fórmula de la $W(u)$ permite obtener resultados en forma automática y con precisión asegurada. El error relativo en todo el rango de aplicación práctica de su argumento $\left(10^{-5}<\mathrm{u}<1\right)$ se mantiene por debajo del $1 \%$. Además, como ya se adelantó, se convierte en una herramienta muy útil a la hora de considerar sistemas heterogéneos más complejos que utilizan a la función $W(u)$ como base de cálculo. 
En la siguiente figura se comparan las expresiones consideradas en contraste con los valores tabulados de la $\mathrm{W}(\mathrm{u})$ que también pueden ser obtenidos a partir del desarrollo en serie presentado en (23) con suficiente cantidad de términos (con $\mathrm{m}=100$ no hay diferencia con los valores tabulados hasta el cuarto decimal).

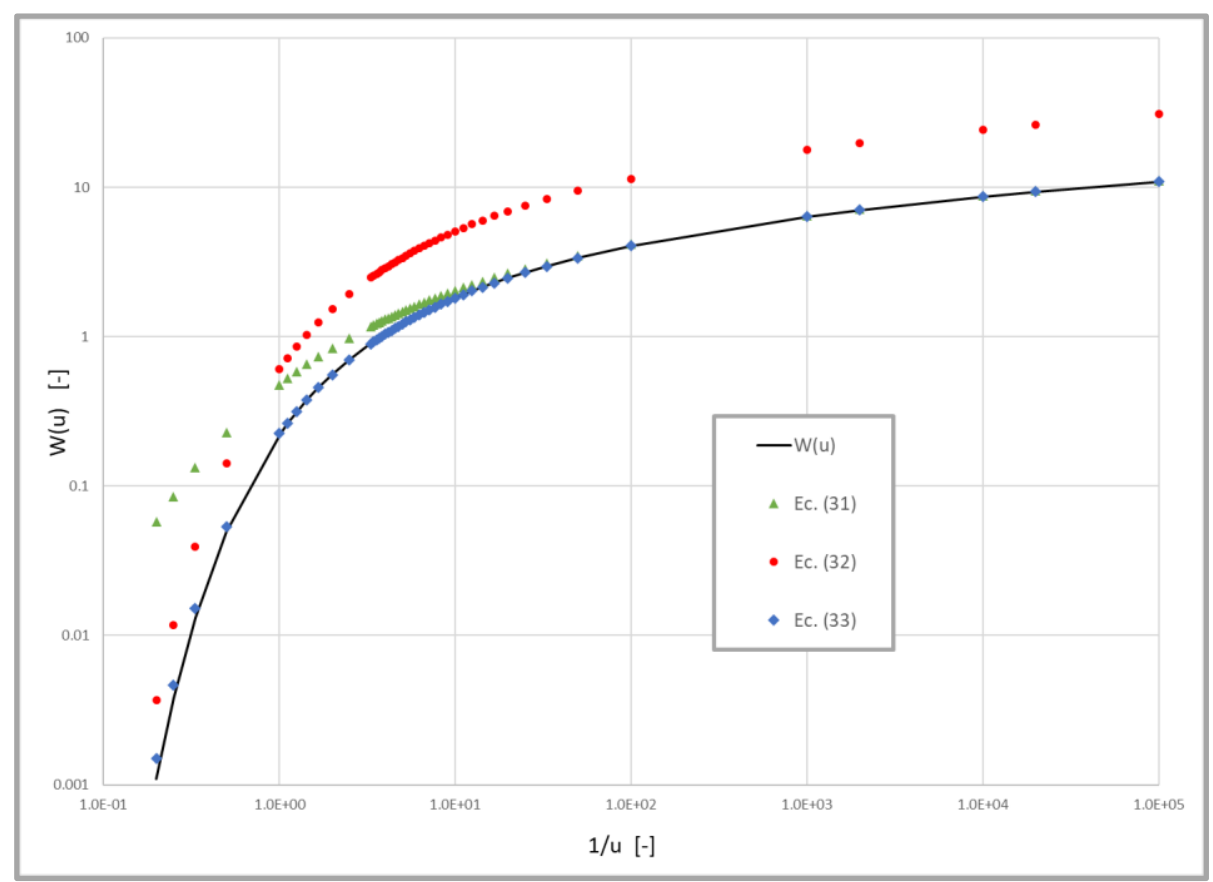

Figura 2.5: Comparación de distintas aproximaciones a la función de pozo $W(u)$.

En la Figura 2.5 se ha adoptado el formato tradicional de graficar la función adimensional $W(u)$ versus la inversa de su argumento $(1 / u)$. En la misma se puede apreciar que la fórmula (33) acompaña a la solución exacta en todo el rango de la abscisa, desde $1 / u=0,2(u=5)$ hasta $1 / u=100.000\left(u=10^{-5}\right)$.

\subsubsection{Modelo de doble porosidad por analogía con la teoría de Hantush.}

Se puede proceder por analogía si se asimila que en una formación multicapa los acuíferos cumplen el rol de las fisuras con espesores reducidos y que los mantos acuitardos que producen su semi-confinamiento se comportan como la matriz en un medio con doble porosidad. Para un caso simple de una sola capa semiconfinante (Hantush, 1964; Kazemi, 1969) la ecuación diferencial que se aplica es:

$$
\left(\frac{\partial^{2} d}{\partial r^{2}}+\frac{1}{r} \frac{\partial d}{\partial r}\right)=\frac{S}{T} \frac{\partial d}{\partial t}+\frac{d}{B^{2}}
$$

donde el significado físico de cada uno de sus términos se conserva igual a lo definido en la (20), excepto que ahora es importante destacar que tanto la trasmisividad $\mathrm{T}=\mathrm{T}_{\mathrm{f}}$, como el coeficiente de almacenamiento $S=S_{f}$, se refieren a la capa acuífera (fisura en esta analogía) y se agrega el denominado "factor de goteo" (B) que se define como:

$$
B=\sqrt{\frac{T_{f}}{K_{i} / b^{\prime}}}
$$

donde $B[L]$ es el factor de goteo; $T_{f}\left[L^{-2} T\right]$ es la trasmisividad de la fisura; $K_{i}\left[L^{-1}\right]$ y $b^{\prime}[L]$ propiedades ya definidas anteriormente para el bloque matriz. 
Siguiendo los mismos pasos que en el punto anterior (aplicación de la Transformada de Laplace bajo las condiciones de borde e iniciales impuestas, obtención de la ecuación diferencial ordinaria en el plano de Laplace, solución general y determinación de sus coeficientes) se llega a la siguiente expresión:

$$
D(r, p)=\frac{Q}{2 \pi T_{p}} \frac{K_{0}\left(r \sqrt[p]{\frac{p S_{f}}{T_{f}}+\frac{1}{B^{2}}}\right)}{r_{w} \sqrt[p S_{f}]{\frac{p}{T_{f}}+\frac{1}{B^{2}}} \cdot K_{1}\left(r_{w} \sqrt{\frac{p S_{f}}{T_{f}}+\frac{1}{B^{2}}}\right)}
$$

Aplicando la inversión de Schapery (Sternberg, 1969) presentada aquí en la expresión (28) y la propiedad de la función $K_{1}$ que simplifica el denominador de la (36), se obtiene una aproximación para este caso que tiene la siguiente forma:

$$
\begin{gathered}
d(r, t)=\frac{Q}{2 \pi T} K_{0}\left(\sqrt{\frac{r^{2} S_{f}}{2 t T_{f}}+\frac{r^{2}}{B^{2}}}\right)=\frac{Q}{2 \pi T} K_{0}\left(\sqrt{2 u+\left(\frac{r}{B}\right)^{2}}\right)=\frac{Q}{4 \pi T} W_{1}\left(u, \frac{r}{B}\right) \\
W_{1}\left(u, \frac{r}{B}\right)=2 K_{0}\left(\sqrt{2 u+\left(\frac{r}{B}\right)^{2}}\right)
\end{gathered}
$$

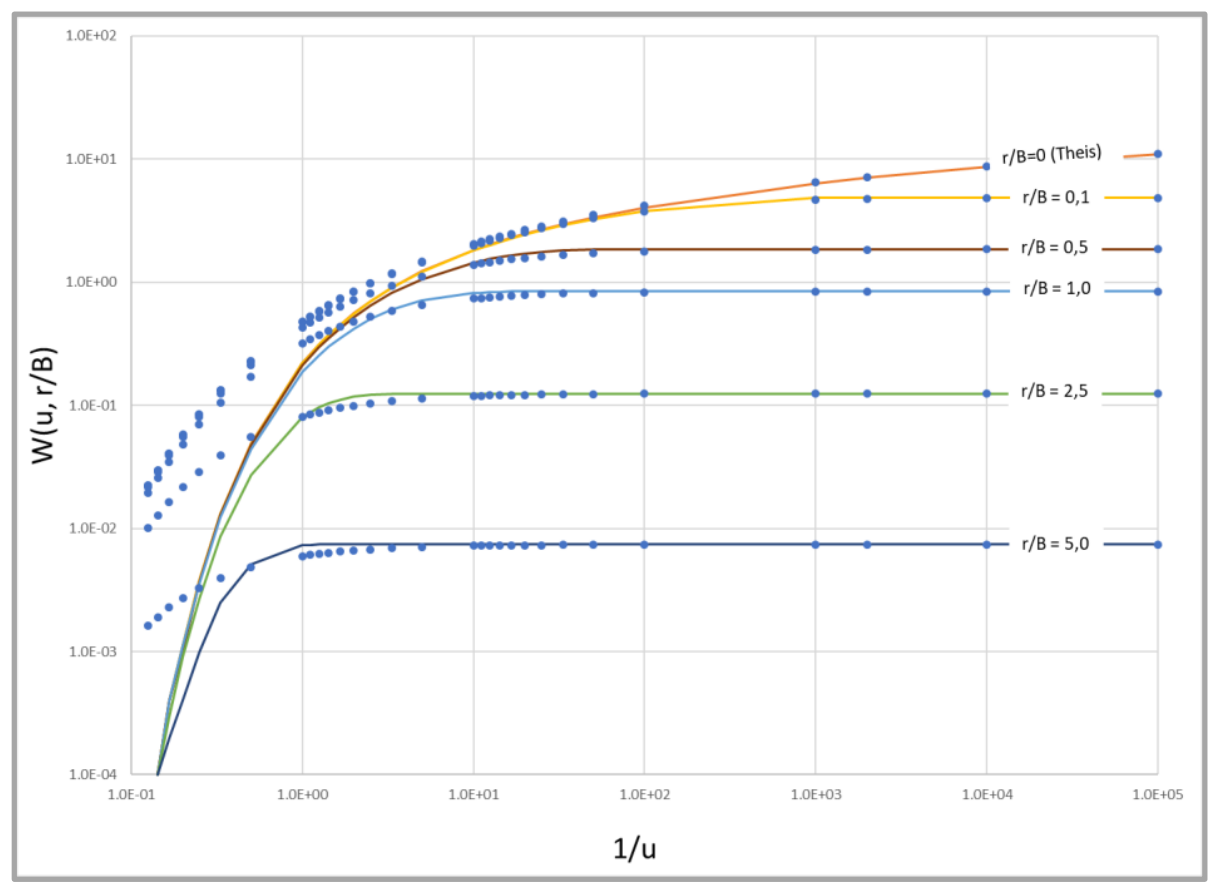

Figura 2.6: Función de pozo de Hantush $W(u, r / B)$ y su aproximación mediante la ec. (38).

Como se puede apreciar en la figura anterior, la fórmula aproximada (38) se ajusta bien (marcas con círculos) a los valores tabulados de la función de Hantush (curvas con trazo continuo) para valores de $u<0,1(1 / u>10)$.

Es preciso destacar que este modelo de acuífero semiconfinado representa un caso particular y límite para un medio análogo con doble porosidad en aquellas situaciones donde pueda suponerse que el coeficiente de almacenamiento de la matriz $\left(S_{m}\right)$ es infinito.

En el caso más general, el aporte desde la matriz porosa provoca un aporte más lento y por ende una pendiente variable de la función de pozo hasta alcanzar un nuevo régimen tipo Theis pero con la combinación de aportes de ambos almacenamientos (matriz + fisura). 
Cuando en el modelo de acuífero semiconfinado de Hantush (1964) no se puede despreciar el efecto del almacenamiento en el acuitardo (equivalente en el modelo de doble porosidad con $S_{m}$ finito) las curvas tipo se dividen en dos segmentos (Custodio y Llamas, 1996):

- Para tiempos largos $\left(\mathrm{t}>2 \mathrm{~b}^{\prime} \mathrm{S}_{\mathrm{m}} / \mathrm{K}_{\mathrm{i}}\right)$ :

$$
d(r, t)=\frac{Q}{4 \pi T} W_{1}\left(u^{*}, \frac{r}{B}\right)
$$

donde $\mathrm{u}^{*}=\mathrm{u}(1+\phi / 3) \operatorname{con} \phi=\mathrm{S}_{\mathrm{m}} / \mathrm{S}_{\mathrm{f}}$.

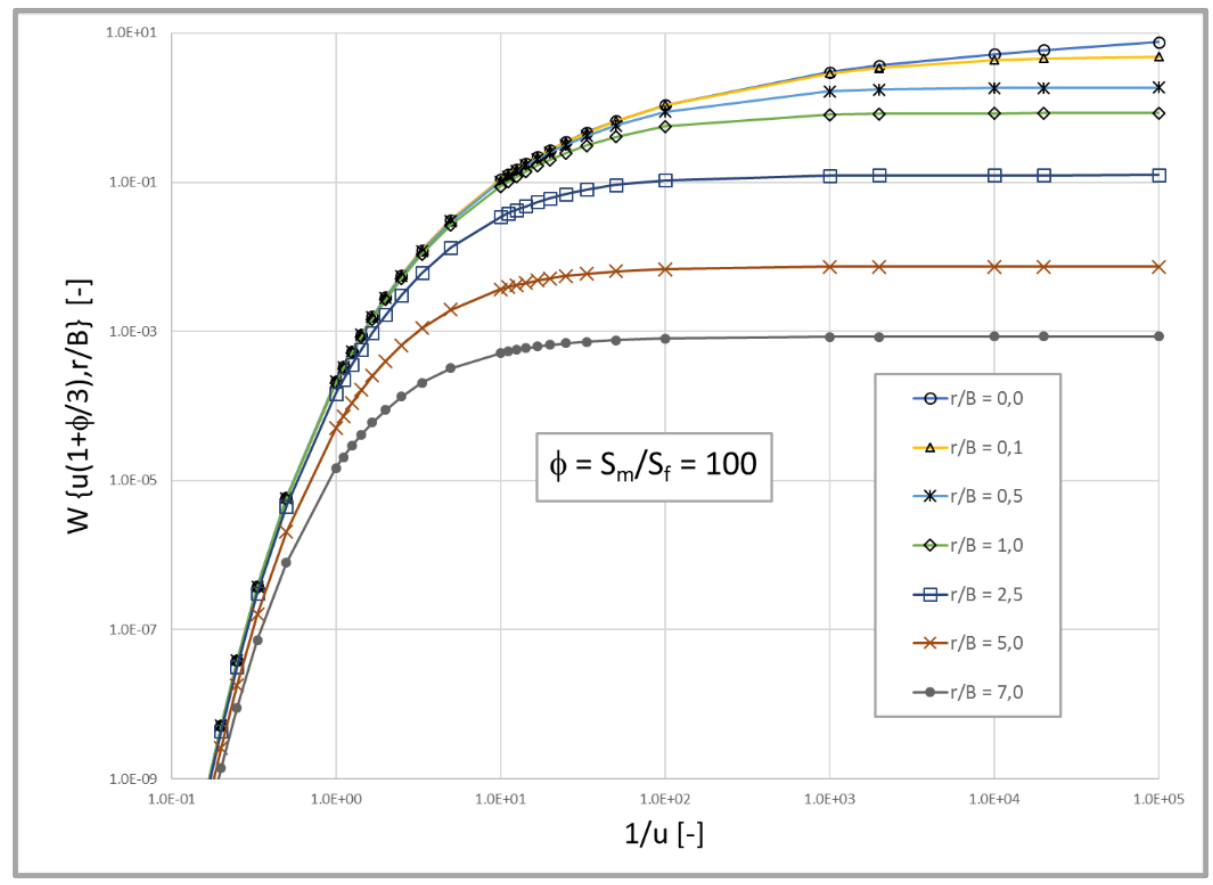

Figura 2.7: Función de pozo de Hantush $W\left(u^{*}, r / B\right)$ para $\phi=S_{m} / S_{f}=100$

- Para tiempos cortos $\left(t<b^{\prime} S_{m} / 10 K_{i}\right)$ :

$$
d(r, t)=\frac{Q}{4 \pi T} H(u, \rho)
$$

donde $H(u, \rho)$ es la función de Hantush y $\rho=\frac{r}{4 B} \sqrt{\frac{S_{m}}{S_{f}}}=\frac{r}{B} \frac{\phi^{1 / 2}}{4}$. La función $H(u, \rho)$ se encuentra tabulada, pero puede ser aproximada con la expresión (Custodio y Llamas, 1996):

$$
H(u, \rho) \cong W(u)-\frac{4 \rho}{\sqrt{\pi u}}\left(0,258+0,693 e^{-u / 2}\right) \quad\left(\text { para } u>10^{4} \rho^{2}\right)
$$

$$
\text { O con: } \quad H(u, \rho) \cong 0,5 \ln \left(\frac{0,044}{\rho^{2} u}\right) \quad \text { (válida para } u<10^{-4} \rho^{2} \text { ) }
$$

El comportamiento temporal de esta función se puede apreciar en la próxima figura 2.8. De cualquier modo, lo que se intenta rescatar aquí son las condiciones en los límites que aporta esta analogía independientemente de lo cedido por el acuitardo (matriz en nuestro caso): por un lado, a corto plazo se debería esperar un comportamiento como se muestra en la figura 2.8; a largo plazo, dependiendo del volumen (importancia) del acuitardo (matriz) un proceso que tiende a equilibrar la extracción por bombeo (curva horizontal en las figuras 2.6 y 2.7 ). 


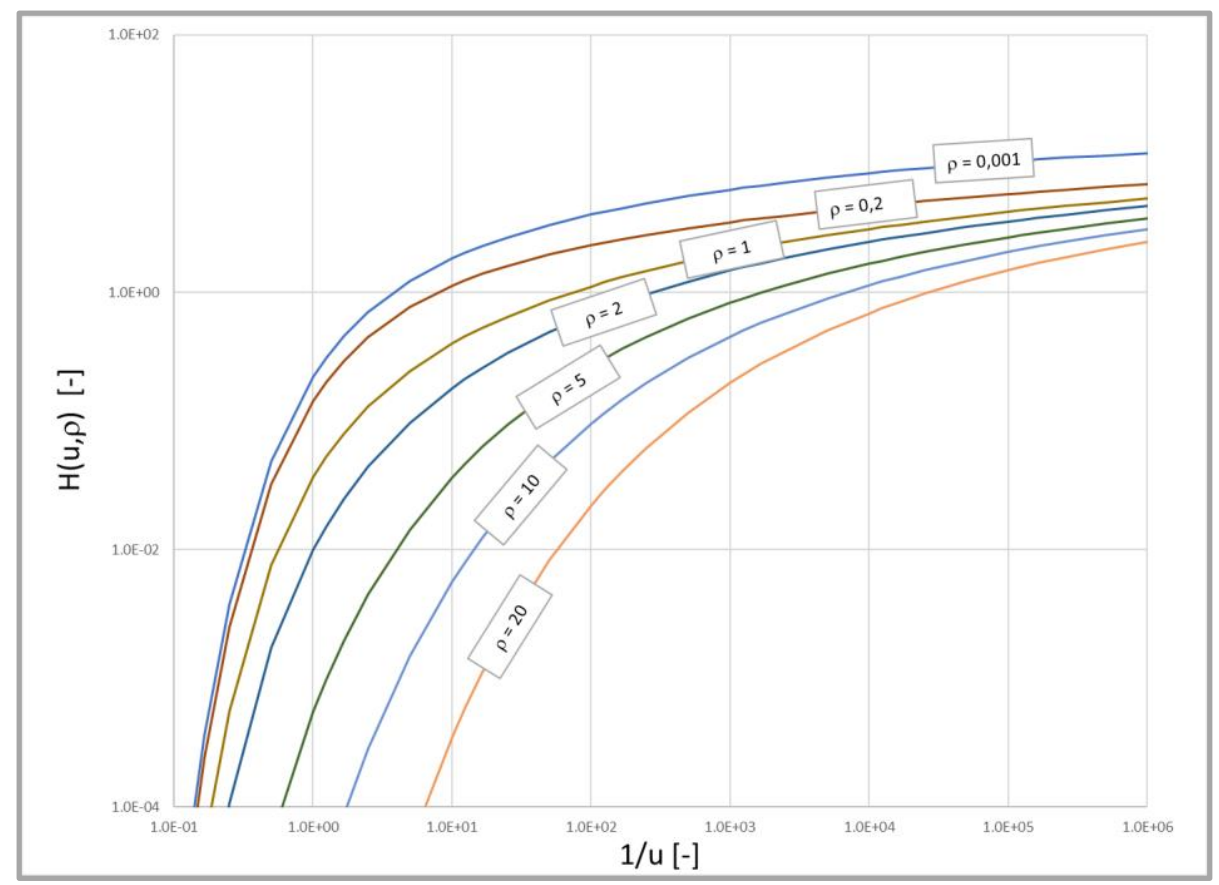

Figura 2.8: Función de pozo de Hantush $H(u, \rho)$ para tiempos cortos.

\subsubsection{Modelo clásico de acuífero confinado con doble porosidad Barenblatt (caso A)}

Si del modelo general de flujo en acuífero confinado con doble porosidad, expresado en las ecuaciones (6) y (7) se desprecia la compresibilidad del sistema de fisuras debido a su baja participación en el volumen total del acuífero entonces se puede despreciar el primer sumando del segundo miembro de la expresión (6). Luego, combinando las ecuaciones resultantes, aplicando las condiciones de borde e iniciales ya señaladas para flujo radial a un pozo central bombeado a caudal constante (Streltsova, 1978) se obtiene una solución del tipo:

$$
d_{f}=\frac{Q}{4 \pi T} \int_{0}^{\infty} J_{0}(x r)\left[1-e^{\left(\frac{k t x^{2}}{1+x^{2} B_{1}^{2}}\right)}\right] \frac{d x}{x}
$$

donde:

- $\quad d_{f}[L]$, es el descenso en las fisuras;

- $\quad$ Q, T, r es el caudal, la Trasmisividad y la distancia radial al pozo, ya definidos;

- Jo es la función de Bessel de primera especie y orden 0;

- $\quad B_{1}=(T / C)^{1 / 2}[L]$, una nueva definición del factor de goteo con $C$ coef. de la FTMF;

- $\quad \kappa=T / S_{m}\left[L T^{-1}\right]$, difusividad o coeficiente de difusión;

- $\quad$ x, una variable de integración.

La integración de (43) se puede realizar con métodos numéricos y como opción se puede acceder a tablas o curvas tipo de la función de pozo $W_{1}$ en función de $r / B_{1}$, como sigue: 


$$
d_{f}=\frac{Q}{4 \pi T} W_{1}\left(u_{m}\right) \quad \text { con } \quad u_{m}=\frac{S_{m} r^{2}}{4 T t}[-]
$$

Este es el modelo de doble porosidad original (Barenblatt et al., 1960) que presenta curvas tipo tal cual se muestra en la siguiente figura:

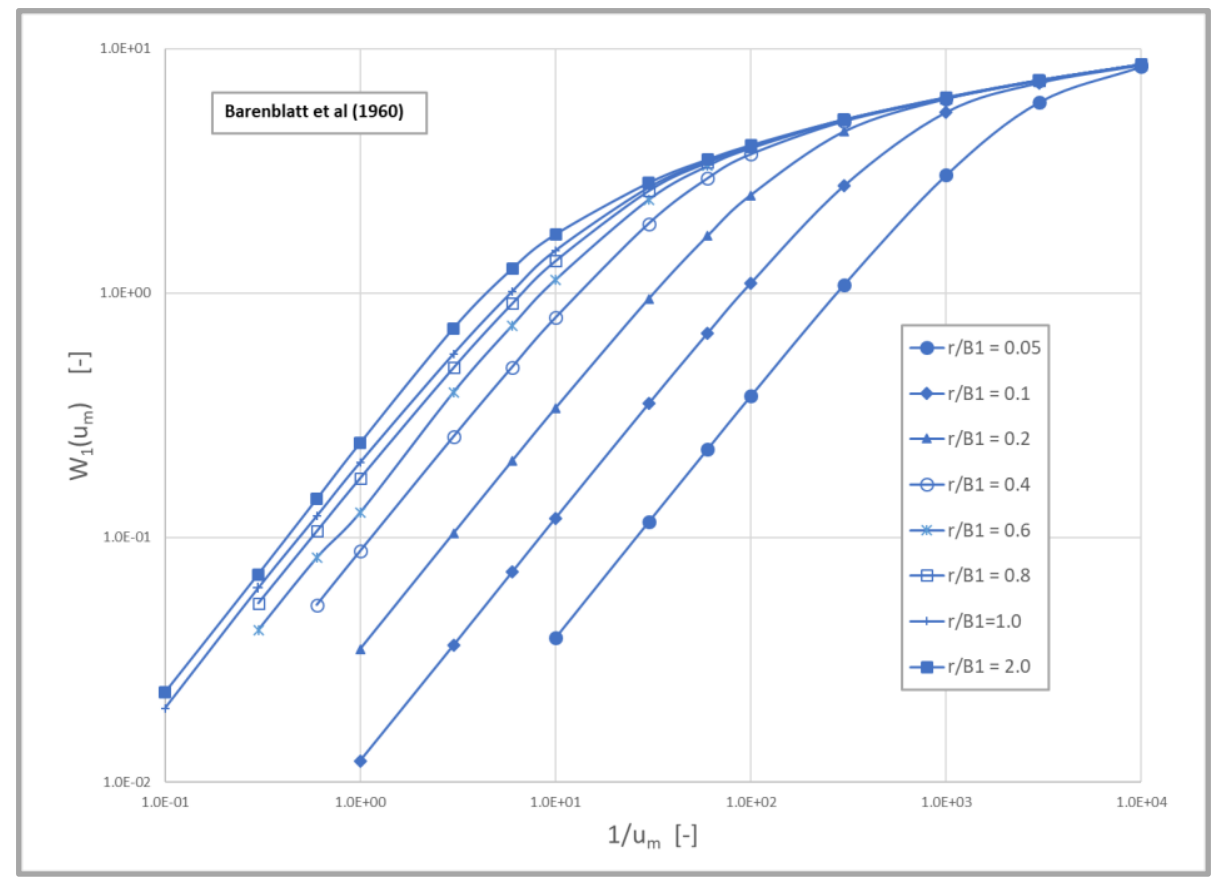

Figura 2.9: Función de pozo de Barenblatt, $W_{1}\left(u_{m}\right)$ para acuíferos con doble porosidad.

Cada curva tipo inicia con un tramo recto que denuncia la hipótesis asumida respecto de la rigidez infinita que se le asignó al sistema de fisuras. Se puede demostrar que la pendiente inicial de cada curva tipo de Barenblatt responden a:

$$
\frac{\partial d_{f}}{\partial t}=\frac{Q}{2 \pi T} \frac{K}{B_{1}^{2}} K_{0}\left(\frac{r}{B_{1}}\right)
$$

donde todos los términos han sido definidos con anterioridad. Como se desprende de (45), cada inicio de curva tipo depende de la relación $r / B_{1}$, es decir, que para tiempos cortos (inmediatos al inicio del bombeo) la pendiente de la recta de Barenblatt es predecible con la (45) en una gráfica doble logarítmica.

\subsubsection{Modelo de acuífero confinado con fracturas horizontales (caso B).}

El modelo de acuífero confinado con doble porosidad es ahora un medio estratificado donde cada bloque matricial es horizontal y de espesor $\mathrm{H}=2 \mathrm{~b}^{\prime}$, limitados por capas de fisuras de pequeño espesor $h$. Se trata entonces de combinar las ec. (6) y (7) para obtener una única ecuación sistema de la siguiente forma:

$$
T\left(\frac{\partial^{2} d_{f}}{\partial r^{2}}+\frac{1}{r} \frac{\partial d_{f}}{\partial r}\right)=S_{f} \frac{\partial d_{f}}{\partial t}-S_{m} \frac{\partial d_{m}}{\partial t}
$$

Para la disposición en capas del medio con doble porosidad, Boulton (1963) y Boulton y Streltsova (1977) dieron una solución del tipo: 


$$
d_{f}=\frac{Q}{4 \pi T} W_{2}\left(u_{f}, \frac{r}{B_{2}}, \eta\right)
$$

donde:

- df, $Q, T$ y $r$ tienen el mismo significado físico y dimensiones que antes,

- $\quad W_{2}[-]$, es la función de pozo que en este caso depende de tres parámetros,

- $\mathrm{U}_{\mathrm{f}}=\frac{\mathrm{S}_{\mathrm{f}} \mathrm{r}^{2}}{4 \mathrm{Tt}}[-]$, primer argumento de la función de pozo,

- $B_{2}^{2}=\frac{K_{f}}{K_{i}} h H=\frac{K_{f} h}{K_{i} / H}=\frac{K_{f} h}{K_{i} / 2 b^{\prime}}\left[L^{2}\right]$, una nueva expresión del factor de goteo, $y$

- $\eta=1+\frac{s_{m}}{s_{f}}=1+\phi[-]$, relación de almacenamiento.

En las siguientes figuras se representa la función de pozo $W_{2}$ para distintos valores de $\eta$.

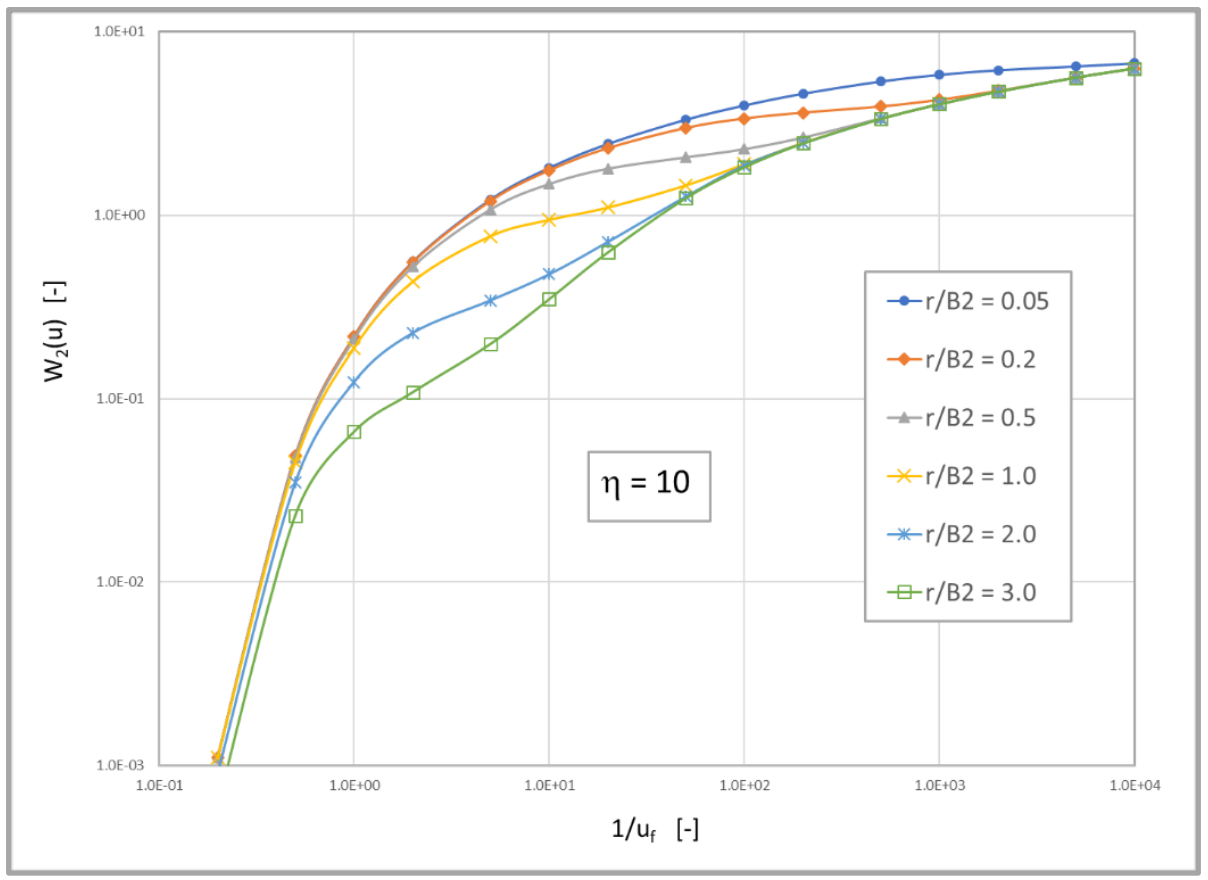

Figura 2.10: Función de pozo de Boulton, $W_{2}\left(u_{f}\right)$ para $\eta=10$.

Es importante notar que en este caso el argumento de la función de pozo $W_{2}$ es $u_{f}$ que depende de parámetros definidos para la componente fisura, y no como en la función de pozo de Barenblatt donde el argumento se combina con la trasmisividad de la fisura $T=T_{f}$ y el almacenamiento de la matriz $\mathrm{S}_{\mathrm{m}}$.

También es destacable que se puede utilizar la conexión del factor de goteo con el coeficiente de la FTMF donde para todos los casos anteriormente planteados podrían responder a una única expresión del tipo $B=(T / C)^{1 / 2}$, donde queda en evidencia que la difusividad del medio queda relacionada a la interfase matriz-fisura gobernada por $\mathrm{C}\left[\mathrm{T}^{-1}\right]$. Allí se encuentra entonces la conexión con el factor de forma $\alpha$, relación que será abordada en el siguiente capítulo. 


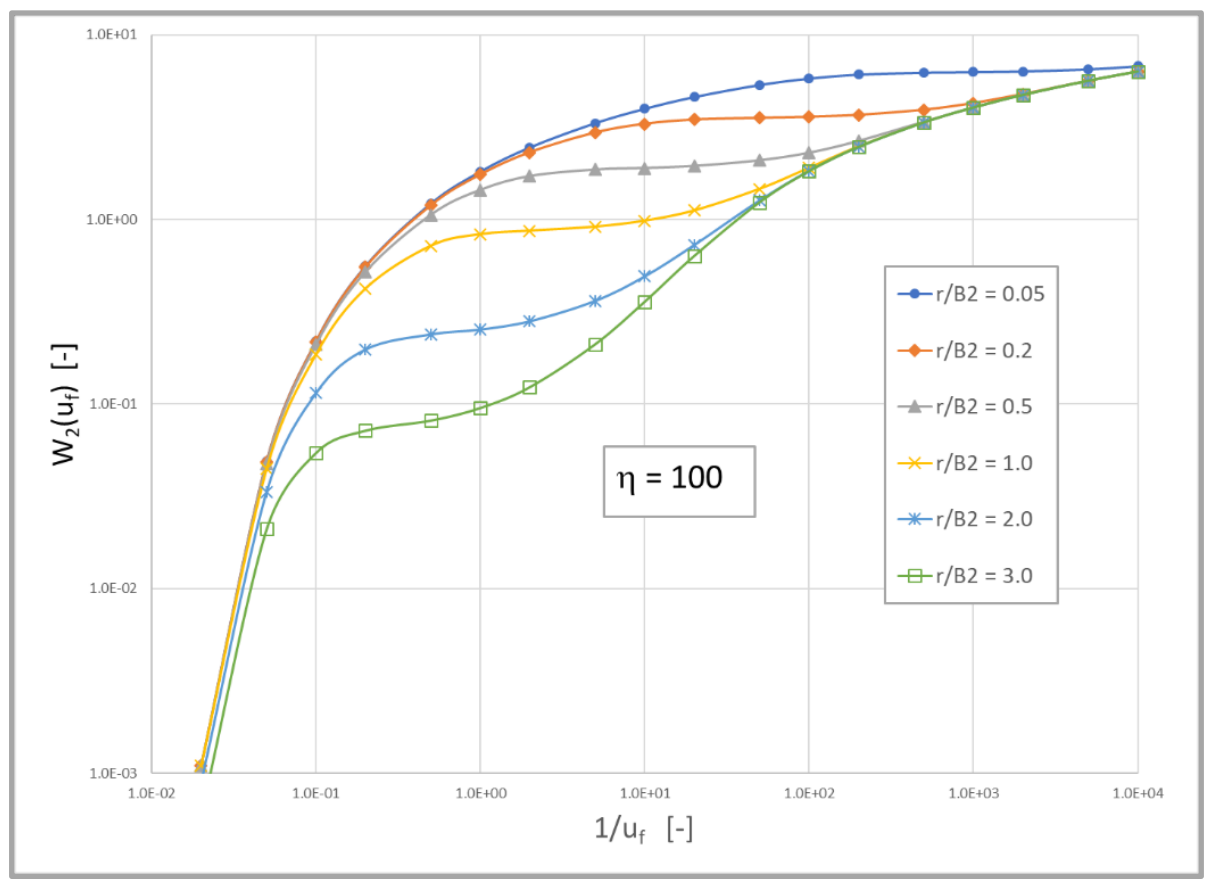

Figura 2.11: Función de pozo de Boulton, $W_{2}\left(u_{f}\right)$ para $\eta=100$.

\subsubsection{Modelo de acuífero confinado con fracturas planas verticales (caso C).}

Un último caso muy típico con solución analítica conocida es para el acuífero atravesado por una única fractura plana vertical pasando por el eje del pozo de bombeo (Gringarten et al. 1974). Con la misma nomenclatura que antes, el descenso relativo adimensional en el pozo de bombeo puede expresarse de la siguiente manera para flujo uniforme en la fractura:

$$
d^{*}=\sqrt{\pi t^{*}} \operatorname{erf}\left(\frac{1}{2 \sqrt{t^{*}}}\right)-\frac{1}{2} E_{i}\left(-\frac{1}{4 t^{*}}\right)
$$

donde:

- $\quad d^{*} \mathrm{y} \mathrm{t}^{*}$ tienen el mismo significado físico y dimensiones como cuando se usaron para definir las ecuaciones (11) y (12).

- erf() es la función de error (integración de la función de la distribución normal)

- $\quad E_{i}$ es la integral exponencial ya definida y que se utilizó para relacionarla con Theis.

De la misma forma, suponiendo que existe una conductividad infinita en la fractura vertical, la expresión centrada en el pozo de bombeo es similar a la anterior y del tipo:

$$
d^{*}=\frac{1}{2} \sqrt{\pi t^{*}}\left[\operatorname{erf}\left(\frac{0.134}{\sqrt{t^{*}}}\right)+\operatorname{erf}\left(\frac{0.866}{\sqrt{t^{*}}}\right)\right]-0.067 E_{i}\left(-\frac{0.018}{4 t^{*}}\right)-0.043 E_{i}\left(-\frac{0.750}{4 t^{*}}\right)
$$

con todos los componentes de esta expresión definidos como se hizo más arriba y con la ventaja de poder usar la expresión (33) para el cómputo de la función integral exponencial $\mathrm{E}_{\mathrm{i}}$.

Las expresiones (48) y (49) también se encuentran tabuladas y a continuación se ilustra cómo se presentan estas ecuaciones en gráfico adimensional en el plano $\left(\mathrm{d}^{*}, \mathrm{t}^{*}\right)$ : 


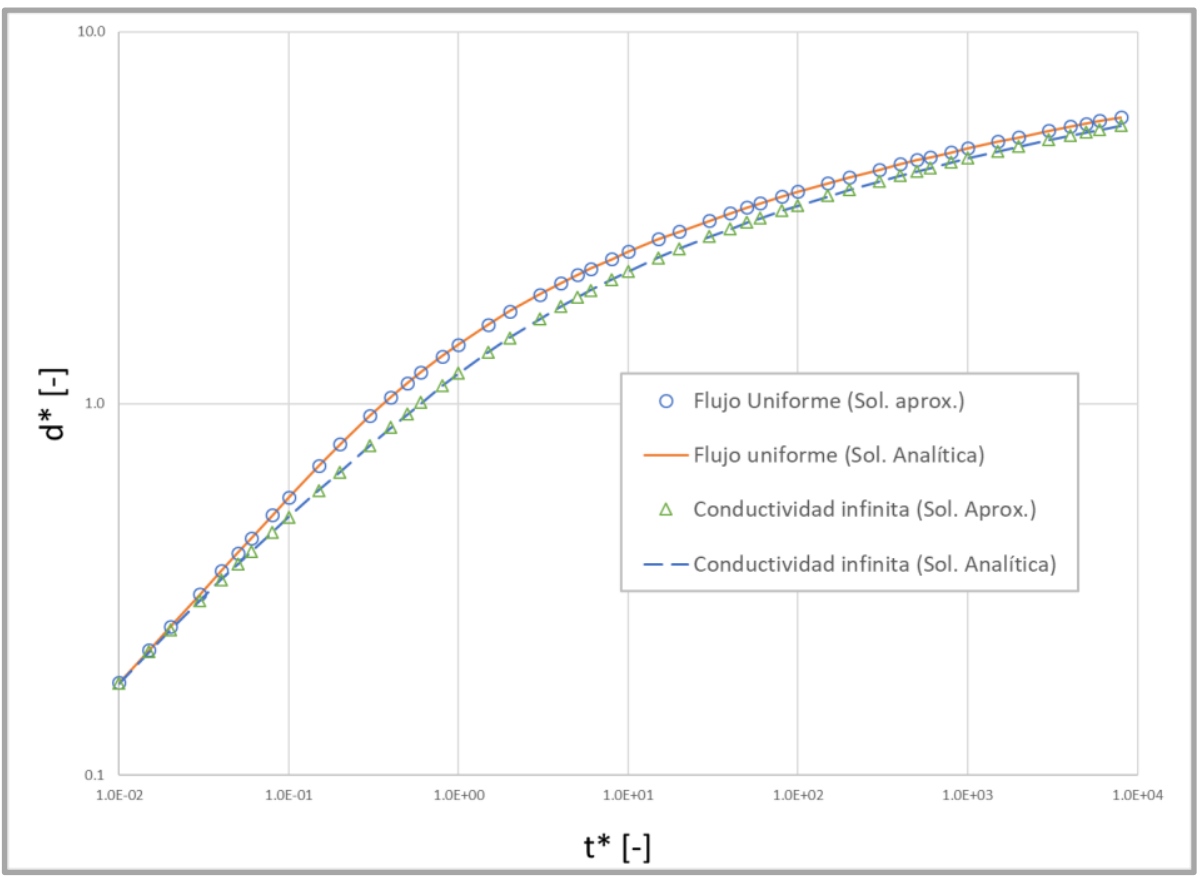

Figura 2.12: Función de pozo para una fractura plana vertical.

\subsubsection{La solución analítica de De Smedt para acuíferos confinados con doble porosidad.}

Como herramienta global de análisis para acuíferos confinados con doble porosidad se incorpora aquí la solución analítica desarrollada por De Smedt (2011), que amplió las hipótesis del modelo de Barenblatt (1960), pero sin contemplar efectos secundarios debidos al almacenamiento en el pozo de bombeo ni el retardo por mineralización o relleno (skin effect) de las fisuras (Moench, 1984).

Como antes visto en este mismo capítulo, partiendo de las ecuaciones generales (6), (7) y (8), se llega a la ecuación de pozo siguiente (De Smedt, 2011):

$$
d=\frac{Q}{4 \pi T} W_{2 P}\left(u, \frac{r}{B}, \phi\right)
$$

donde la función de pozo $W_{2 p}[-]$ que se deriva de la solución analítica desarrollada por De Smedt depende de tres argumentos, a saber:

$$
W_{2 P}\left(u, \frac{r}{B}, \phi\right)=W_{2 P}\left(\frac{s_{f} r^{2}}{4 T t}, \frac{r}{\sqrt{\frac{T}{c}}}, \frac{s_{m}}{S_{f}}\right)
$$

donde se recuerda que $\phi[-]$, cociente entre coeficientes de almacenamiento y $C\left[\mathrm{~T}^{-1}\right]$ es el coeficiente de la función de transferencia definido en (8).

Como bien explica De Smedt en su trabajo, si la matriz no posee capacidad de almacenamiento $\left(S_{m}=0\right)$ o la cesión de agua desde la misma es nula $(C=0)$, la expresión (50) coincide exactamente con la ecuación de Theis (1935) para un acuífero confinado en un medio isótropo y homogéneo (Pinder \& Celia, 2006). 
Por el contrario, si la capacidad de almacenamiento de la matriz es infinita y reacciona instantáneamente a los cambios de presión que provienen de la región de las fracturas, entonces el descenso en el pozo se demora (o se paraliza en caso extremo), derivando en la expresión de Hantush (1964) con B [L] definido como el factor de goteo (Singhal y Gupta, 2010).

Por último, cuando $\mathrm{C}$ tiende a infinito, el sistema vuelve a Theis con un coeficiente de almacenamiento equivalente a la suma de los valores individuales ( $S_{\text {equivalente }}=S_{m}+S_{f}$ ).

\subsection{Herramientas de la Geoestadística para la simulación de medios naturalmente FRACTURADOS}

\subsubsection{Variables regionalizadas, estimación y simulación}

La mayoría de los fenómenos que se estudian en las Ciencias de la Tierra dependen de variables regionalizadas (VR). En general, las VR representan valores que dependen del punto espacial donde se refieren y es la información básica que utiliza la Geoestadística. En efecto, el objeto de la Geoestadística es describir cuantitativamente las variables naturales distribuidas en el espacio (Cassiraga y Gómez-Hernández, 2001). Pero además las VR poseen dos características aparentemente contradictorias (Matheron, 1965):

- manifiestan comportamientos aleatorios (alta irregularidad y variaciones imprevisibles de un punto a otro del espacio), y

- pueden poseer una estructura espacial característica del fenómeno regional.

La teoría de las VR propone desarrollar dos objetivos principales:

- describir matemáticamente esa estructura o continuidad espacial, y

- proponer resoluciones prácticas para la estimación de la VR a partir de una muestra de datos medidos u observaciones del fenómeno regional.

Con respecto a este último objetivo puede anticiparse que el error en el proceso de estimación de la VR será más alto cuanto mayor sea su irregularidad y discontinuidad espacial. Por este motivo es importante destacar que la VR es una realización de una función aleatoria cuya distribución no se conoce a priori. A su vez, una función aleatoria referida a un cierto dominio espacial es un conjunto de variables aleatorias que se corresponden con puntos o posiciones dentro del dominio bajo estudio.

El tipo de información que se puede integrar con los métodos de la Geoestadística pueden ser datos duros/directos de las variables de interés o información blanda/indirecta de variables secundarias (Gómez-Hernández, 1991). La importancia de utilizar como complemento a la información blanda es que puede aportar ventajas al estar disponible en forma exhaustiva en casi todo el campo de estudio.

Los métodos para poder integrar la información dura (casi siempre escasa y en escalas pequeñas) como la información blanda pueden ser clasificados en dos grandes grupos (Cassiraga y Gómez-Hernández, 1996): los algoritmos de interpolación espacial o estimación y los algoritmos de representación estocástica o simulación. 
Para lograr un campo interpolado de la VR, es decir, para lograr exportar una estimación a ubicaciones donde no se ha medido $u$ observado dicha variable se puede utilizar distintas estrategias como por ejemplo la inversa de la distancia al cuadrado o como se hace en Geoestadística: en cada nodo del campo se estima un valor de acuerdo con un criterio de optimización que en la técnica del Krigeado corresponde a minimizar la varianza del error medio cuadrático cometido en cada estimación. Los algoritmos de interpolación producen una sola imagen suave con una varianza mínima que es una medida asociada de su incertidumbre.

Pero también en el campo de la Geoestadística se han desarrollado los algoritmos de simulación estocástica. La simulación estocástica permite generar una serie de campos equiprobables los cuales reproducen los datos medidos y la estructura espacial inferida a partir de ellos. Son realizaciones múltiples del atributo simulado y todas igualmente plausibles. Entonces, dado que todas estas múltiples realizaciones reproducen los datos de partida y el grado de variabilidad detectado, cualquiera podría corresponder con la realidad.

Adoptando la simulación estocástica como método básico que brinda la Geoestadística para atacar el problema de modelar un acuífero con doble porosidad (a partir de la generación de múltiples realizaciones de campos de fisuras o familias de ellas), se distinguen aquí los procedimientos a seguir por medio de la función de probabilidad condicional que se aplica a los dominios bajo estudio.

En principio, una primera división queda establecida en la selección de métodos paramétricos o no paramétricos (Gómez-Hernández y Cassiraga, 1994), para generar la distribución local de probabilidades condicionada a los datos disponibles de partida y a los nodos donde la simulación secuencial vaya actuando.

En principio no se ha querido restringir el análisis de medios con doble porosidad adoptando a priori, por ejemplo, un modelo multigaussiano. Tampoco abunda información de campo que permita confirmar que la porosidad secundaria (en este caso, la distribución espacial de las distintas familias de fisuras) siga ese tipo de distribución probabilística.

Dentro de los métodos no paramétricos y para variables categóricas (incluso dicotómicas como el caso particular de contemplar nodos o bloques diferenciados de matriz y fisura para establecer una representación más aproximada de la geometría del acuífero fracturado), se destaca para el marco de la presente investigación la simulación indicadora secuencial donde no hay un modelo de distribución condicional definido a priori.

A partir de la librería GSLIB (Deutsch y Journel, 1998) y con software SGeMS (Remy et al., 2009) se puede trabajar en campos bidimensionales y tridimensionales con el programa SISIM, que consiste en la aplicación de la técnica de simulación indicadora secuencial en medios discretizados mediante nodos de forma cúbica.

En especial, es conveniente utilizar SISIM para variables categóricas dado que permite diferenciar nodos tipo matriz y tipo fisura con distinto código y adoptar para la simulación distintas proporciones iniciales de ambas componentes. Como ampliación de la librería GSLIB para aplicar técnicas geoestadísticas en tres dimensiones, se dispone también del programa ISIM3D (Gómez Hernández y Srivastava, 1990).

Los algoritmos booleanos son otra opción para estudiar la distribución espacial de variables categóricas. Uno de los procesos booleanos que más se aplica es para la generación de objetos (tal como se mencionara en el apartado 1.2 para el caso de los discos que representan características geométricas de las fisuras en los modelos RFD). 
El proceso desencadenado a través de estos algoritmos sirve para generar un vector de posición con los parámetros aleatorios de la forma elegida (por ejemplo, tamaño, orientación en el espacio, densidad de objetos en un volumen de referencia) para una cierta categoría (p.e. familia k-ésima de fracturas). Suelen mencionarse en la bibliografía como procesos aleatorios de marcación puntual, es decir, marcar el punto aleatoriamente elegido con una señal, mojón o bandera, en este caso con un objeto con atributos o características aleatorias.

Para generar formas elípticas con un algoritmo booleano se cuenta en la librería del GSLIB con el programa ELLIPSIM. Las aplicaciones de este programa y de SISIM en el presente trabajo de tesis se detallan en los próximos puntos.

\subsubsection{Simulación indicadora secuencial con el programa SISIM}

Dentro de la gran variedad de técnicas de simulación existente, la simulación secuencial es una de las más conocida y aplicada. La simulación secuencial (Alabert, 1987; Journel, 1989; Isaaks, 1990; Gómez-Hernández y Srivastava, 1990; Gómez-Hernández, 1991; GómezHernández y Journel, 1993; Gómez-Hernández y Cassiraga, 1994), permite generar tanto campos multinormales como no. En ambos casos es posible la simulación de variables continuas y categóricas. De los algoritmos no multinormales, los más utilizados son los que se basan en una aproximación indicadora (Alabert, 1987; Journel y Alabert, 1988; Gómez-Hernández y Srivastava, 1990).

Considerando la simulación de la distribución espacial de $K$ categorías $s_{k}$ mutuamente excluyentes, condicionadas a un conjunto de datos $\left\{s\left(\mathbf{u}_{\alpha}\right), \alpha=1, \ldots, n\right\}$, el algoritmo de simulación indicadora secuencial puede ser descrito a través de los siguientes pasos:

- Transformar cada dato categórico $s\left(\mathbf{u}_{\alpha}\right)$ en un vector de $K$ indicadores duros de acuerdo con la definición de la variable indicadora, esto es:

$$
i\left(\mathbf{u}_{\alpha} ; s_{k}\right)=\left\{\begin{array}{l}
1 \text { si } s\left(\mathbf{u}_{\alpha}\right)=s_{k} \\
0 \text { si no }
\end{array} \quad k=1, \ldots, K\right.
$$

- Definir una secuencia aleatoria de visita a los nodos de la malla.

- En cada nodo de coordenadas u donde se debe generar un valor de la variable:

1) Determinar la probabilidad condicional de ocurrencia de cada categoría sk, utilizando (co)kriging simple u ordinario. La información condicionante está integrada por los datos y los valores previamente simulados.

2) Corregir las probabilidades estimadas para evitar problemas en las relaciones de orden.

3) Definir algún orden de las K categorías y construir la función de distribución acumulando las correspondientes probabilidades de ocurrencia.

4) Generar un número aleatorio $p$ con distribución uniforme entre 0 y 1 y muestrear la función de distribución acumulada. La categoría simulada para el nodo $\mathbf{u}$ es aquella que corresponde al intervalo de probabilidad dentro del cual está p.

5) Agregar el valor generado a los datos que condicionarán la generación de los nodos siguientes.

6) Continuar con el siguiente nodo y repetir los pasos 1) a 5). 
En esta tesis se utiliza el algoritmo de simulación indicadora secuencial de variables categóricas para la generación de simulaciones no condicionadas en las que nos interesa respetar una cierta proporción en las categorías.

En consecuencia, el algoritmo parte de un conjunto de datos vacío, esto es, se generan simulaciones no condicionadas. La información de partida es el número de categorías, la proporción de cada una y la estructura espacial de las mismas.

Por ejemplo, para la generación de una familia de fisuras en un bloque de dimensiones de $(100 \times 100 \times 50)$ cubos elementales de dimensión unitaria $(1 \times 1 \times 1)$, se ingresaron en el programa SGeMS (Remy et al., 2009) los siguientes parámetros:

- Grilla: 100, 100, 50 celdas con dimensiones 1,1,1 y origen $0,0,0$

- Variable: categórica con dos umbrales 0.10, 0.90 (según proporción de fisuras)

- Estimación: IK medio (Krigeado indicador)

- $\quad$ Datos: sin datos

- Vecindario de búsqueda: 100, 100, 50 con ángulos 0,0,0.

- Variograma: 0 de pepita, meseta 1, modelo Gaussiano, Rangos 2000, 2000, 0.5

- Ángulos de generación (orientación de familia de fisuras): 0,0,0 (horizontal)

Como se puede notar, se han exagerado los ejes de anisotropía del Variograma en el plano $(X, Y)$ y se ha propuesto un modelo Gaussiano para poder lograr un plano de fractura lo más continuo posible. Los resultados para distintos ángulos de generación se presentan en la siguiente figura:

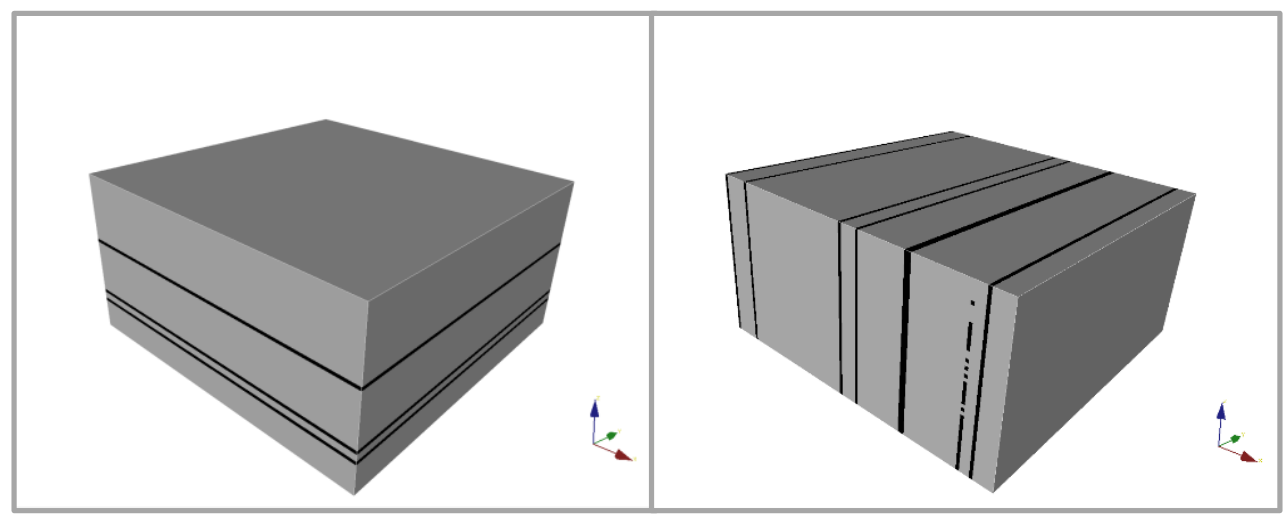

Figura 2.13: Bloques con fracturas horizontales $(0,0,0)$ y verticales $(90,90,0)$ con SISIM.

En cuanto a la continuidad de los planos de fracturas sólo se busca una condición ideal o básica cuando se conoce que en la realidad los distintos tipos de fisuras no siempre son planos (pueden tener curvatura) ni son paralelas las superficies que definen sus límites (pueden presentar puntos/zonas de contacto).

Una comparación de los tres tipos de modelos de variograma disponibles en el programa SGeMS da como resultado apreciable que, como se adelantó, el modelo Gaussiano produce realizaciones con mayor cantidad de planos continuos de fisuras, le sigue el modelo Exponencial y por último el modelo Esférico. 
En la siguiente figura se comparan los distintos modelos de variogramas aplicados a los mismos datos de partida para generar una familia vertical de fracturas. También se muestra una familia oblicua a los ejes coordenados (orientación 0,45,45).

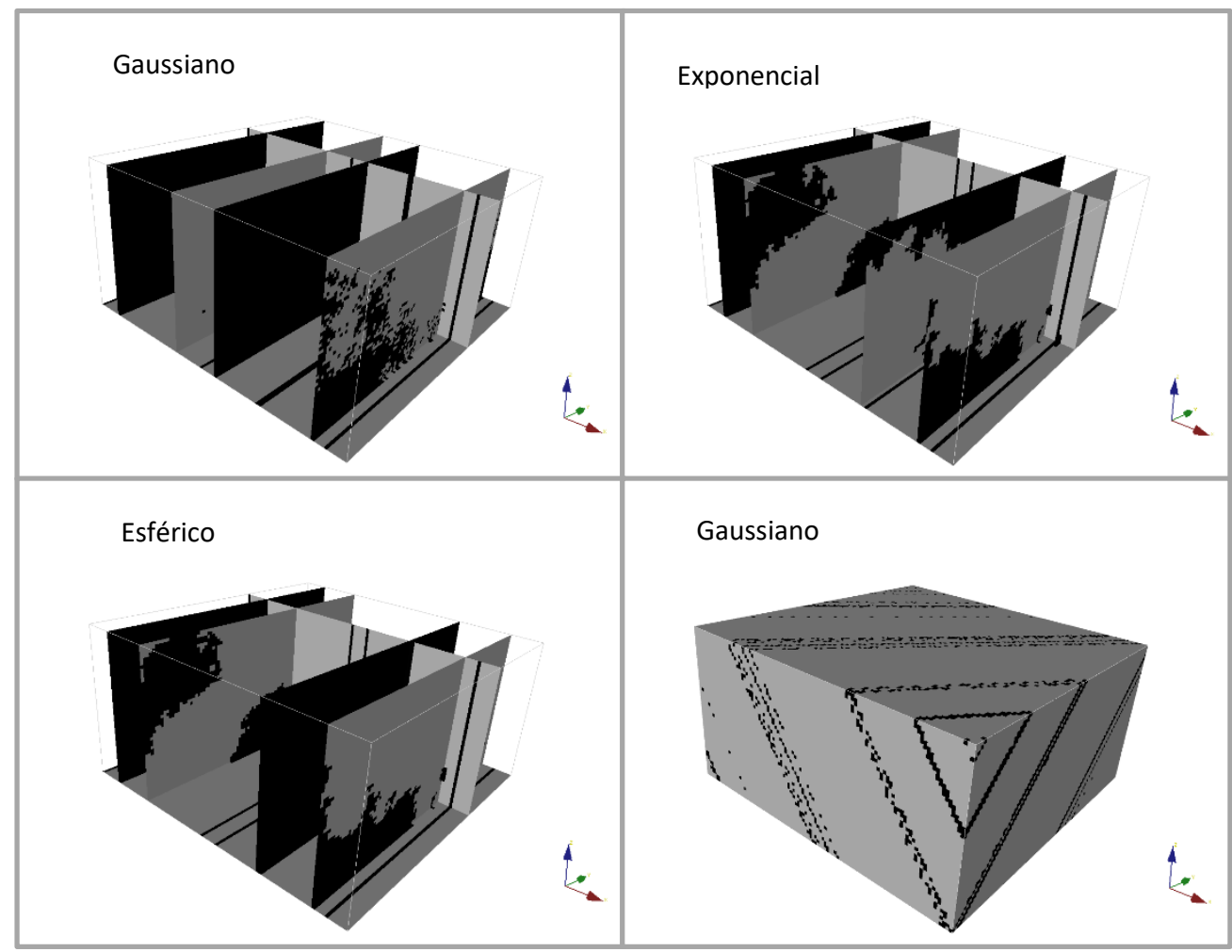

Figura 2.14: Comparación de bloque generados con distintos modelos de variogramas.

En general, la práctica de la generación de familias de fracturas con este procedimiento depende de la cantidad de familias a generar y la proporción que ocupan las mismas en el volumen del bloque. Siguiendo recomendaciones al respecto (Cassiraga y Gómez-Hernández, 2001) las familias de fracturas se generan por separado y luego se combinan en un mismo bloque. Para cumplir con esta consigna se desarrolló un programa específico para el manejo de este tipo de información y cuyo código se presenta en el Anexo 8.3 de esta tesis.

\subsubsection{Simulación con algoritmos booleanos - El programa ELLIPSIM}

Este método puntual de simulación de familias de fracturas en la práctica presentó algunas características que resultan destacables:

- Es muy sencillo de utilizar. Un ejemplo de entrada de datos se ha incluido en el Anexo 8.3.

- Permite generar varias familias al mismo tiempo sin dificultad. Se trabajó con la rutina ELLIPSIM de GSLib directamente (sin utilizar SGeMS ya que no se encuentra dentro del menú de opciones para simulación).

- En general respeta muy bien la proporción solicitada de fracturas en relación con el volumen total.

- Genera planos continuos de fracturas que atraviesan el bloque simulado. Esto facilita la reproducción de familias de fracturas con orientaciones regionales que siempre suelen estar presentes y son más identificables que los patrones (no ya tan continuos) de pequeñas fisuras locales. 
No obstante, el ajuste a datos de campo no es ya tan expeditivo (Deutsch y Journel, 1998) y suele advertirse que la calibración con los datos de campo disponible para reproducir un cierto patrón espacial es "un arte" y tiende a ser resuelto por prueba y error.

En el marco de los ensayos realizados en el presente trabajo esto no ofició como una dificultad porque sólo se tuvieron en cuenta las orientaciones de las familias y su proporción con referencia al volumen total del bloque.

Un resumen del algoritmo ELLIPSIM puede ser representado por la siguiente secuencia de operaciones:

\section{Programa ELLIPSIM}

i. Lee el nombre del archivo de datos

ii. Lee los parámetros del archivo de datos:

a. Número de realizaciones a simular

b. Origen, dimensiones del cubo elemental y tamaño del bloque

c. Número semilla para el generador aleatorio

d. Proporción de elementos booleanos (\% del volumen de elipses)

e. Características geométricas, orientación y peso del elemento

iii. Verificación de especificaciones y control de límites del bloque, rotación y anisotropía de las elipses

iv. Ciclo para todas las realizaciones hasta alcanzar la proporción deseada de elipses con respecto al volumen total del bloque:

a. Elección aleatoria de los ejes de la elipse

b. Elección aleatoria de la posición del centro de la elipse

c. Relleno de la elipse con la categoría elegida para ello (0 ó 1)

d. Vuelta al paso a. de este ciclo para generar otra elipse.

Finaliza el programa ELLIPSIM

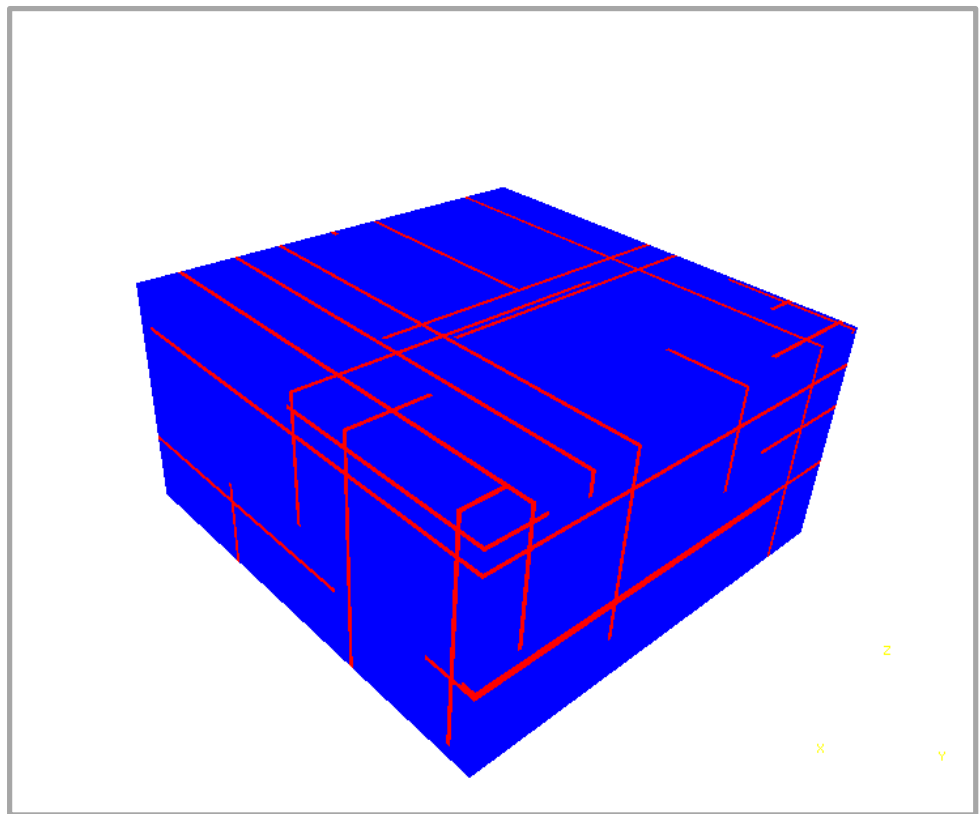

Figura 2.15: Una realización de 3 familias de fracturas generadas con ELLIPSIM (10\% en volumen). 


\section{MÉtOdO COMBINADO PARA EVALUAR ACUífEROS CAUTIVOS CON DOBLE POROSIDAD}

De acuerdo con lo expuesto en los capítulos precedentes, se presenta aquí un método para realizar la interpretación de un ensayo de bombeo cuando se sospecha una clara incidencia de la doble porosidad en los resultados obtenidos o cuando se posee evidencia de la estructura dual en la unidad acuífera ensayada a través de trabajos previos de reconocimiento en campo.

Esta metodología intenta proveer una herramienta práctica y sencilla para enriquecer el análisis de la interpretación de los datos experimentales obtenidos por medio de ensayos de bombeo y confrontarlos no sólo con las curvas tipo correspondientes a una aproximación determinística de un caso complejo, sino que avanza también en la posibilidad de obtener un aprovechamiento mejor del conocimiento de la estructura geológica del acuífero.

En general existe siempre una gran incertidumbre acerca de cómo está configurada la red de fracturas en un acuífero, pero al menos siempre se tiene la posibilidad de reconocer en campo las principales familias constituyentes y la orientación espacial de las mismas (azimut / rumbo, dirección e inclinación del buzamiento y rake/giros en el plano de falla). Con esta información y estimando la proporción de fracturas en relación con el volumen unitario de roca, es posible generar familias de fracturas con las herramientas de simulación estocástica que ofrece la Geoestadística.

\subsection{PRIMER PASO: CÓMPUTO DEL FACTOR DE FORMA CON AYUDA DE LA GEOESTADÍSTICA}

Para poder computar el factor de forma $\alpha\left[\mathrm{L}^{-2}\right]$ que integra la FTMF es necesario generar primero un conjunto de $n$-familias de fracturas en un bloque y simular luego varias realizaciones manteniendo la orientación y la proporción con respecto al volumen total del mismo.

Para esto se pueden utilizar las herramientas ELLIPSIM y SISIM de la librería GSLib (Deutsch y Journel, 1998) con la ayuda del programa SGeMS (Remy et al., 2009) para el control y la visualización de los resultados.

Cuando se cuenta con el ensamble de las realizaciones se necesita calcular el factor de forma $\alpha\left[\mathrm{L}^{-2}\right]$ como el cuadrado de la relación Superficie de intercaras vs. Volumen total del bloque simulado. En este trabajo se optó por desarrollar una rutina (escrita y compilada en fortran95) que resuelva primero la tarea de combinar en un mismo bloque las distintas familias de fisuras y que luego compute la cantidad de intercaras matriz-fisura. En el Anexo 8.3 y en el DVD adjunto a esta tesis se incluyen ejemplos de los programas empleados para este fin.

El factor de forma así calculado cumple un rol fundamental a la hora de evaluar el coeficiente $\boldsymbol{C}\left[\mathrm{T}^{-1}\right]$ de la FTMF. Esta forma de trabajo permite trabajar la mayoría de los ejemplos estudiados con la solución de De Smedt (2011) a efectos de mejorar la aproximación de esta fórmula a los datos observados de un ensayo de bombeo.

Finalmente, dado que no siempre se encuentra a disposición la orientación y proporción de familia de fisuras, se desarrollaron casos testigos que puedan servir de guía para poder llevar adelante este primer paso. En los puntos que siguen se resumen las elaboraciones realizadas para obtener funciones que vinculen al factor de forma con la orientación, el número de familias y la proporción de las mismas en el volumen total de la formación acuífera. Se trabajó con ambos programas (SISIM y ELLIPSIM) a efectos de comprobar el comportamiento de cada uno. 


\subsubsection{Generación booleana de familias de fracturas con ELLIPSIM}

Para tener un espectro adecuado de realizaciones con esta herramienta de simulación, se adoptaron distintas configuraciones de 1,2 y hasta 3 familias de elipses planas cuyos ejes de anisotropía se ubicaron perpendiculares a los ejes coordenados. Asimismo, se trabajó con distintos porcentajes de volumen de fisuras, variando desde $1 \%$ hasta el $25 \%$.

Para cada conjunto de familias (previamente combinadas en un mismo bloque) se simularon 100 realizaciones. En la figura 3.1 se muestra el resultado final del cálculo del factor de forma $\alpha$ en función de número de familias y el porcentaje de fisuras en el bloque.

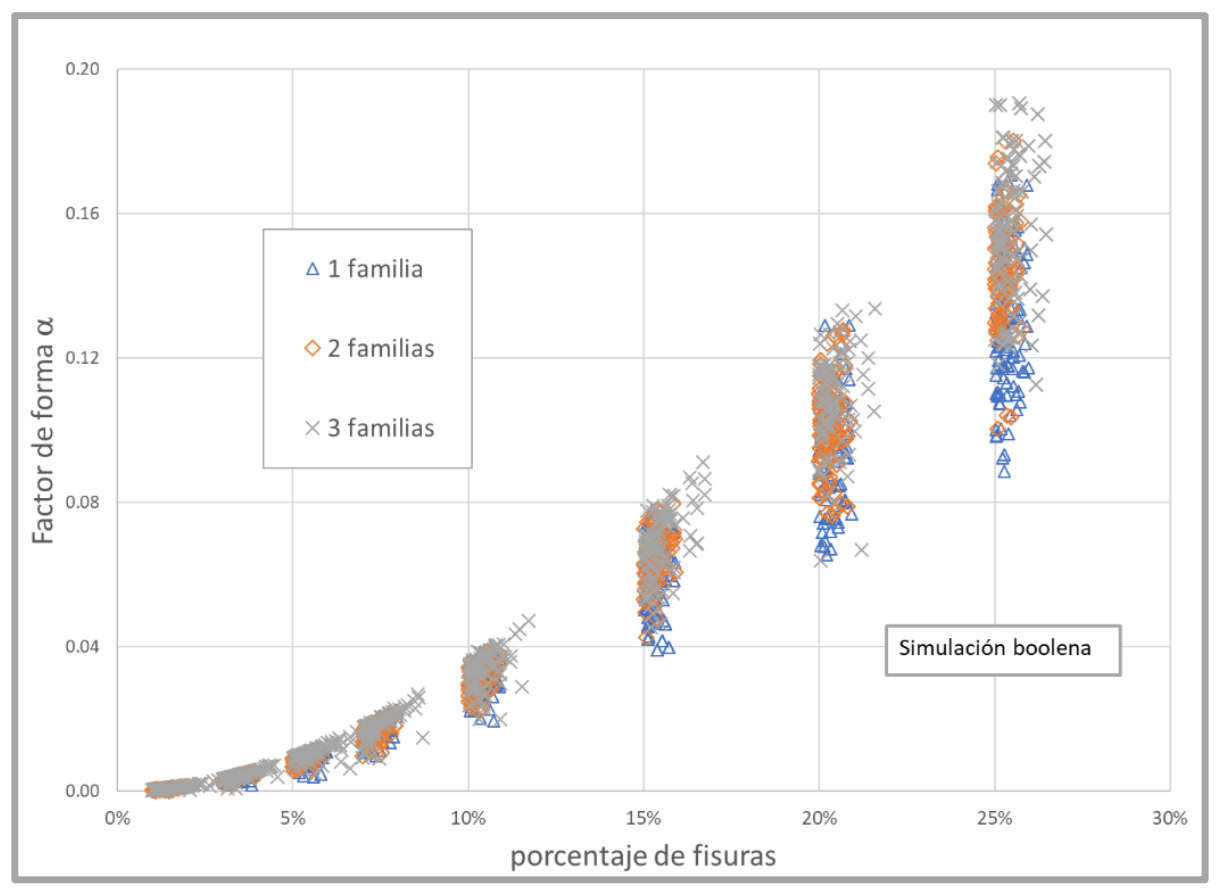

Figura 3.1: Tres familias de fracturas para el cálculo del factor de forma con ELLIPSIM.

Las distintas realizaciones de las familias de fracturas simuladas en un bloque de $100 \mathrm{x}$ $100 \times 50$ cubos de $1 \mathrm{~m}$ de lado, dan origen a un espectro de valores del factor $\alpha$, tal cual se muestra en la Figura 3.1. En la misma puede apreciarse que, a medida que el porcentaje volumétrico de fisuras aumenta, el factor de forma se incrementa con una ley de tipo potencial o cuadrática dependiendo del grado de ajuste deseado. Hay también mayor dispersión de resultados a medida que las familias y el porcentaje de fisuras aumenta, posiblemente debido al mayor número de intersecciones que se generan.

\subsubsection{Generación de familias de fracturas con SISIM}

En este caso, para la generación de familias de fracturas se empleó el algoritmo de simulación indicadora secuencial SISIM, en modo variable categórica. El bloque utilizado tiene las mismas dimensiones $(100 \times 100 \times 50)$ que en el caso anterior. La proporción volumétrica de las fracturas se hizo variar entre 5 y $25 \%$. El resultado del Factor de forma (100 realizaciones para cada simulación) en función del porcentaje y la cantidad de familias ortogonales de fisuras se muestra en la figura siguiente: 


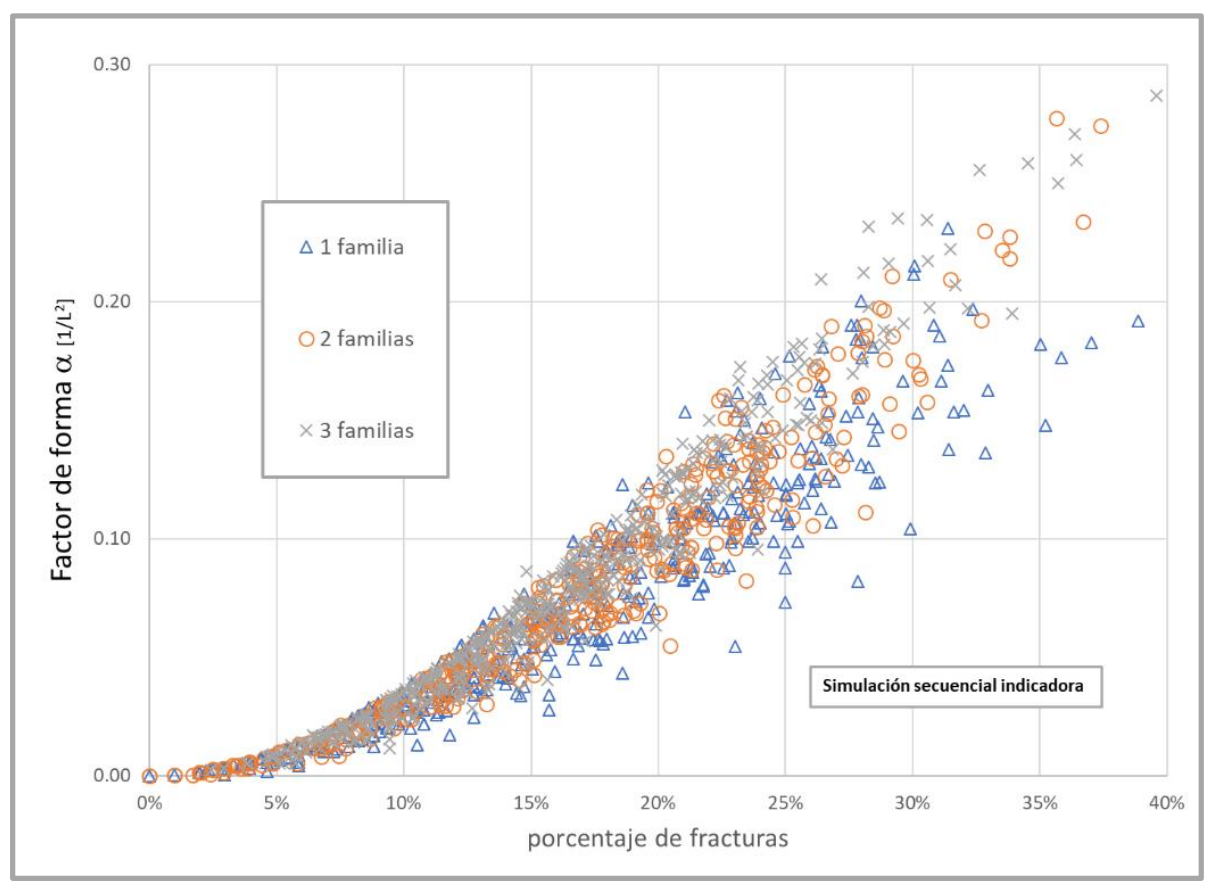

Figura 3.2: Tres familias de fracturas para el cálculo del factor de forma con SISIM.

Aquí se comprueba mucha mayor dispersión de resultados en los valores del factor de forma como consecuencia de la mayor variabilidad estocástica que genera el algoritmo SISIM.

\subsubsection{Relación del factor de forma con la proporción de fisuras}

Existen variadas referencias acerca de la estimación del factor de forma de la FTMF (Lim y Aziz, 1995; Hassanzadeh et al., 2006 y 2009; Ranjbar et al., 2011). En todas las fórmulas de cálculo prevalece como consigna básica la definición del número de familias de fracturas o dimensiones que posee el flujo. Por el contrario, poco se hace referencia a la proporción volumétrica del sistema de fracturas en el área de influencia del pozo de bombeo.

Por ejemplo, en el trabajo de Ranjbar y Hassanzadeh (2011) se presenta el rango de variación más recientemente publicado sobre el factor de forma $\alpha$. Dependiendo de las dimensiones del flujo (1D, 2D o 3D), el coeficiente a aplicar a las dimensiones del bloque que sirve como referencia varía según se muestra en la siguiente tabla:

Tabla 3.1: Rango de variación del coeficiente de bloque (de Ranjbar y Hassanzadeh,2011)

\begin{tabular}{ccc}
\hline Dimensión del flujo & Límite mínimo & Límite máximo \\
\hline 1D & 4 & 12 \\
2D & 8 & 32 \\
3D & 12 & 60 \\
\hline
\end{tabular}

De esta manera, aplicando estos límites de la Tabla 3.1 a la expresión de la longitud equivalente $L_{e}^{2}=\Sigma\left(1 / L_{i}\right)^{2}$ (con $i=1$ a 3 ) para un bloque de referencia como fuera usado en esta trabajo de Tesis $(100 \times 100 \times 50)$ se obtiene: 
Tabla 3.2: Rango del factor de forma para un bloque de $100 \times 100 \times 50$

\begin{tabular}{ccc}
\hline Dimensión & Límite mínimo & Límite máximo \\
\hline 1D & 0,00040 & 0,0012 \\
2D & 0,00040 & 0,0016 \\
3D & 0,00053 & 0,0027 \\
\hline
\end{tabular}

Si se comparan con los rangos de las simulaciones obtenidas con ELLIPSIM y SISIM (conservando estas orientaciones ortogonales entre sí, es decir, sin considerar rumbo ni buzamiento) estos valores corresponderían a valores muy bajos de proporción volumétrica de fisuras en el bloque elegido para la simulación. Esto significa que el espesor otorgado a toda la transición matriz-fisura es muy pequeño $\left(b^{\prime}\right)$, prácticamente nulo.

Como aporte inédito se presentan expresiones analíticas para tener en cuenta este factor manteniendo ortogonales las familias de fisuras para que sirvan de referencia. Las expresiones son las siguientes:

$$
\begin{array}{lll}
\text { 1D } & - & \alpha=1,28 \mathrm{p}^{1,64} \\
2 \mathrm{D} & - & \alpha=1,46 \mathrm{p}^{1,66} \\
3 \mathrm{D} & - & \alpha=1,83 \mathrm{p}^{1,74}
\end{array}
$$

donde $\mathrm{p}$ es la proporción de fracturas en el volumen de control. En las gráficas siguientes se representan los ajustes alcanzados. La correlación de los factores de forma computados con las 100 realizaciones generadas por SISIM ha sido muy buena para los tres casos desarrollados.

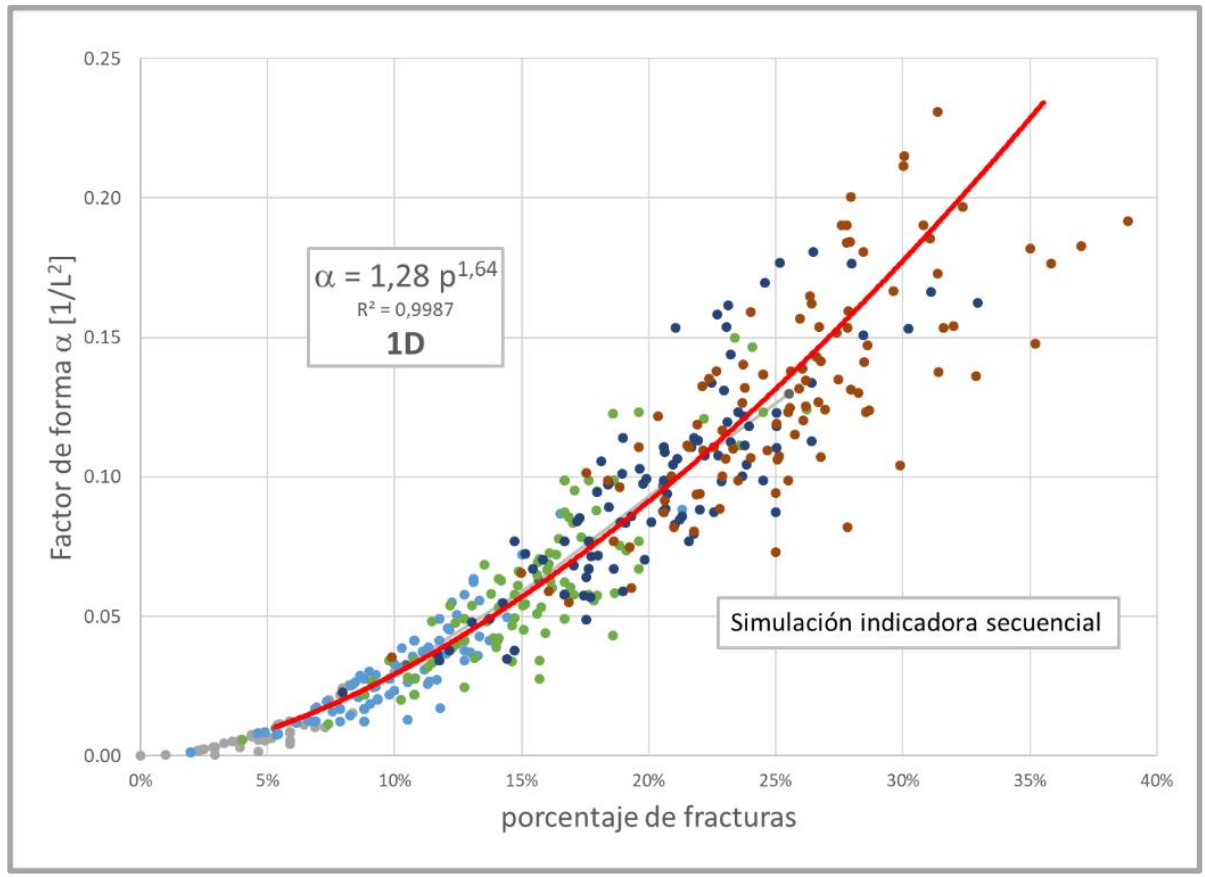

Figura 3.3: Factor de forma en función de la proporción de fisuras (1D).

Para casos reales más complicados (como el abordado en el capítulo siguiente) y dado que las posibilidades podrían resultar infinitas, se recomienda generar directamente las realizaciones con SISIM con el objetivo de obtener luego un valor del factor de forma promedio del ensamble. 


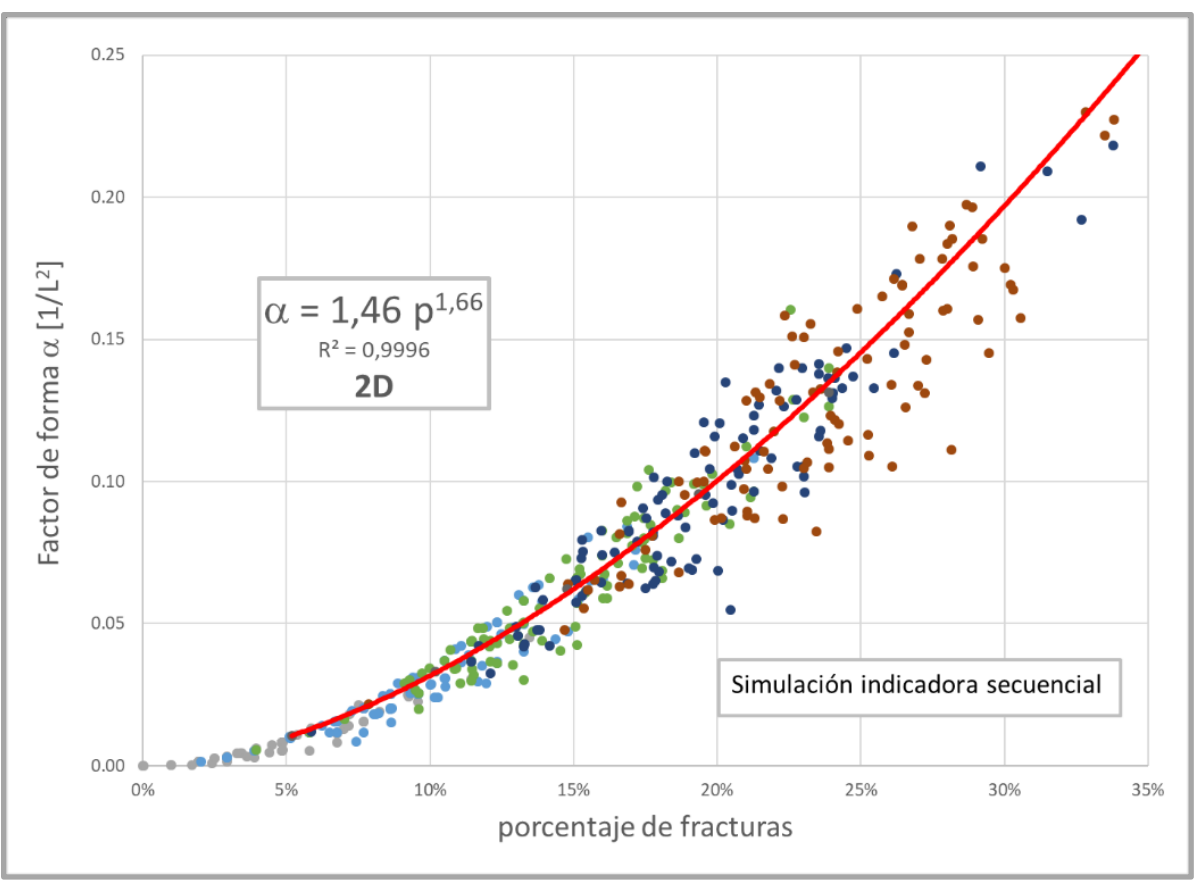

Figura 3.4: Factor de forma en función de la proporción de fisuras (2D).

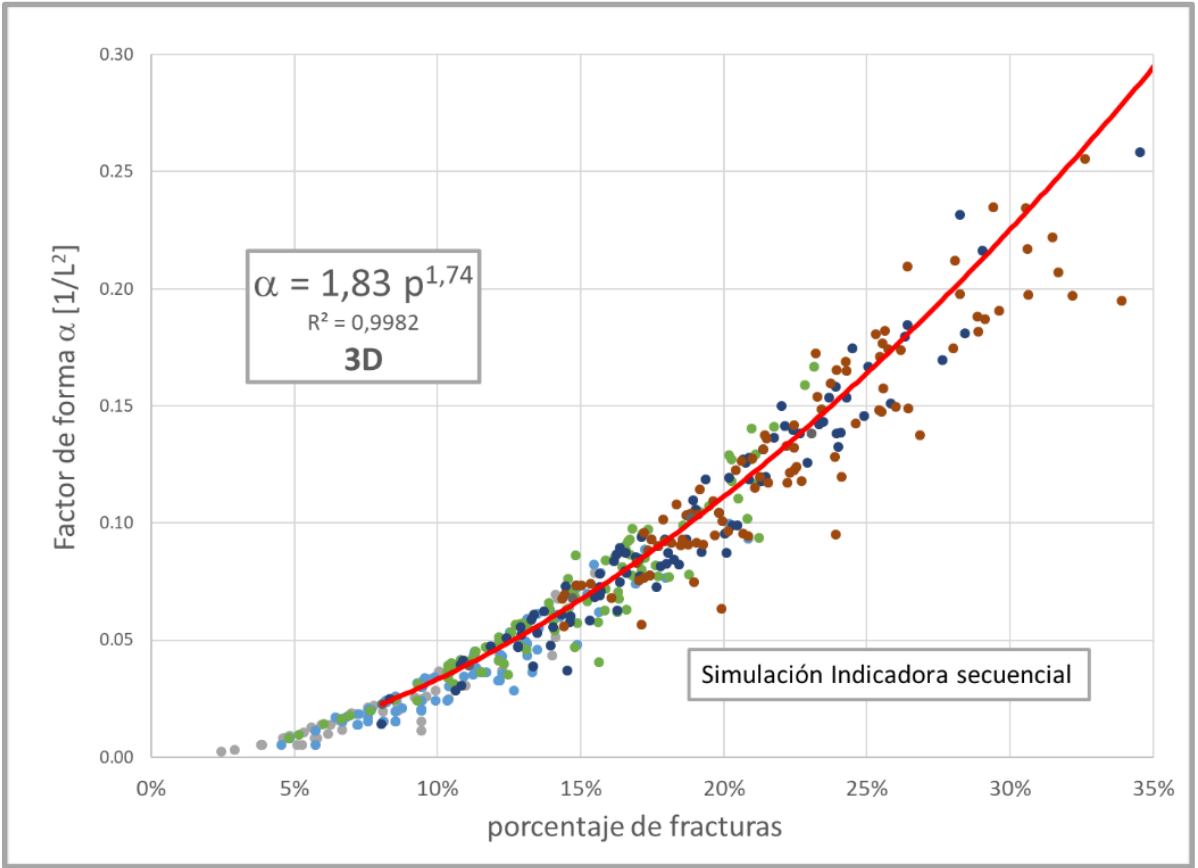

Figura 3.5: Factor de forma en función de la proporción de fisuras (3D).

Con estas relaciones ajustadas a una función potencial se tiene una herramienta rápida para estimar el valor del factor de forma $\alpha$ y del coeficiente $C$ de la FTMF. Esto no es menor si se tiene en cuenta que es muy laborioso trabajar con SISIM y combinar las familias en un solo bloque. La desventaja de usar esta aproximación es que las familias se han orientado sólo en forma perpendicular a los ejes coordenados, resultando necesario recurrir a SISIM para otras orientaciones más complicadas. 


\subsection{SEGUNDO PASO: EVALUACIÓN DE LOS PARÁMETROS HIDROGEOLÓGICOS.}

\subsubsection{Procedimiento de De Smedt para la interpretación de ensayos de bombeo}

En su trabajo (De Smedt, 2011) incluye una rutina de ajuste de los parámetros de la ecuación (50) a las observaciones de los descensos en los pozos de observación en un ensayo de bombeo. La rutina se provee bajo la forma de un ejecutable denominado "w2p_optimisation.exe" que corre sobre plataforma Windows (DOS) dado que es un programa compilado en lenguaje Fortran95.

El programa lee todos los datos de entrada desde un archivo de texto denominado "input.txt". A continuación, se reproduce un ejemplo de la misma:

\begin{tabular}{|c|c|c|}
\hline \multicolumn{3}{|c|}{ input.txt - Notepad } \\
\hline File Edit $F$ & Format & View Help \\
\hline \multicolumn{3}{|l|}{ E2 } \\
\hline 1600.0 & $\mathrm{~T}$ & \\
\hline 10.00004 & Sf & \\
\hline 10.002 & $\mathrm{Sm}$ & \\
\hline 10.03 & $\mathrm{C}$ & \\
\hline 0141.42 & $r$ & \\
\hline 0500.0 & $Q$ & \\
\hline 0.000100 & & 0.001000 \\
\hline 0.001000 & & 0.054000 \\
\hline 0.010000 & & 0.073000 \\
\hline 0.052400 & & 0.105300 \\
\hline 0.072200 & & 0.124000 \\
\hline 0.099900 & & 0.138500 \\
\hline 0.500000 & & 0.320000 \\
\hline 0.600000 & & 0.330000 \\
\hline 0.750000 & & 0.340000 \\
\hline 0.802800 & & 0.360000 \\
\hline 0.900500 & & 0.370000 \\
\hline 1.000000 & & 0.380000 \\
\hline
\end{tabular}

Figura 3.6: Ejemplo del archivo de datos para el programa de ajuste de De Smedt.

La primera línea es un título que puede ser propuesto por el usuario. La siguiente línea indica el número de parámetros a optimizar para el ajuste de las observaciones a la ecuación (50); son 6 parámetro y es una constante que no se puede modificar porque esta rutina de optimización ha sido compilada para este problema en particular.

Las siguientes líneas (desde la tercera a la octava) son dedicadas a una estimación inicial de los parámetros precedidos por un código: 1 significa que el parámetro debe ser optimizado, 0 indica que es un parámetro que se desea mantener con su valor actual (sin cambio).

Este último caso puede darse para un parámetro como la distancia radial $(r)$ al pozo de bombeo, siendo este un valor usualmente bien conocido y con bajo nivel de error en su medición por lo cual puede considerarse un dato confiable. Otro parámetro que normalmente no se optimiza es el valor del caudal $Q$ del bombeo. En ambos casos entonces corresponde anteceder al valor el código 0 . Los cuatro parámetros restantes son la trasmisividad $\mathrm{T}$ de la red de fisuras, los coeficientes de almacenamiento de ambas componentes y el coeficiente $\mathrm{C}$ de la FTMF. Las unidades seleccionadas para cada parámetro deben ser coherentes, por ejemplo, si el caudal está dado en $\left[\mathrm{m}^{3} /\right.$ día] entonces será la trasmisividad T [ $\mathrm{m}^{2} /$ día] y el coeficiente $\mathrm{C}$ [1/día]. 
Desde la línea 9 en adelante se vuelcan los valores del ensayo de bombeo en la manera tradicional de una tabla de valores del tiempo desde que se inicia el bombeo y de los descensos observados. La misma recomendación vale en este caso en cuanto a la coherencia del sistema de unidades elegido; en el ejemplo mencionado corresponde entonces anotar los timepos en fracciones de día y los descensos en metros.

Los resultados de este modelo son dados en un archivo aparte denominado "output.txt". En este archivo se van volcando paso a paso las mejoras parciales producidas por el programa de optimización de los parámetros con código 1 y el resultado final con la información estadística del ajuste conseguido:

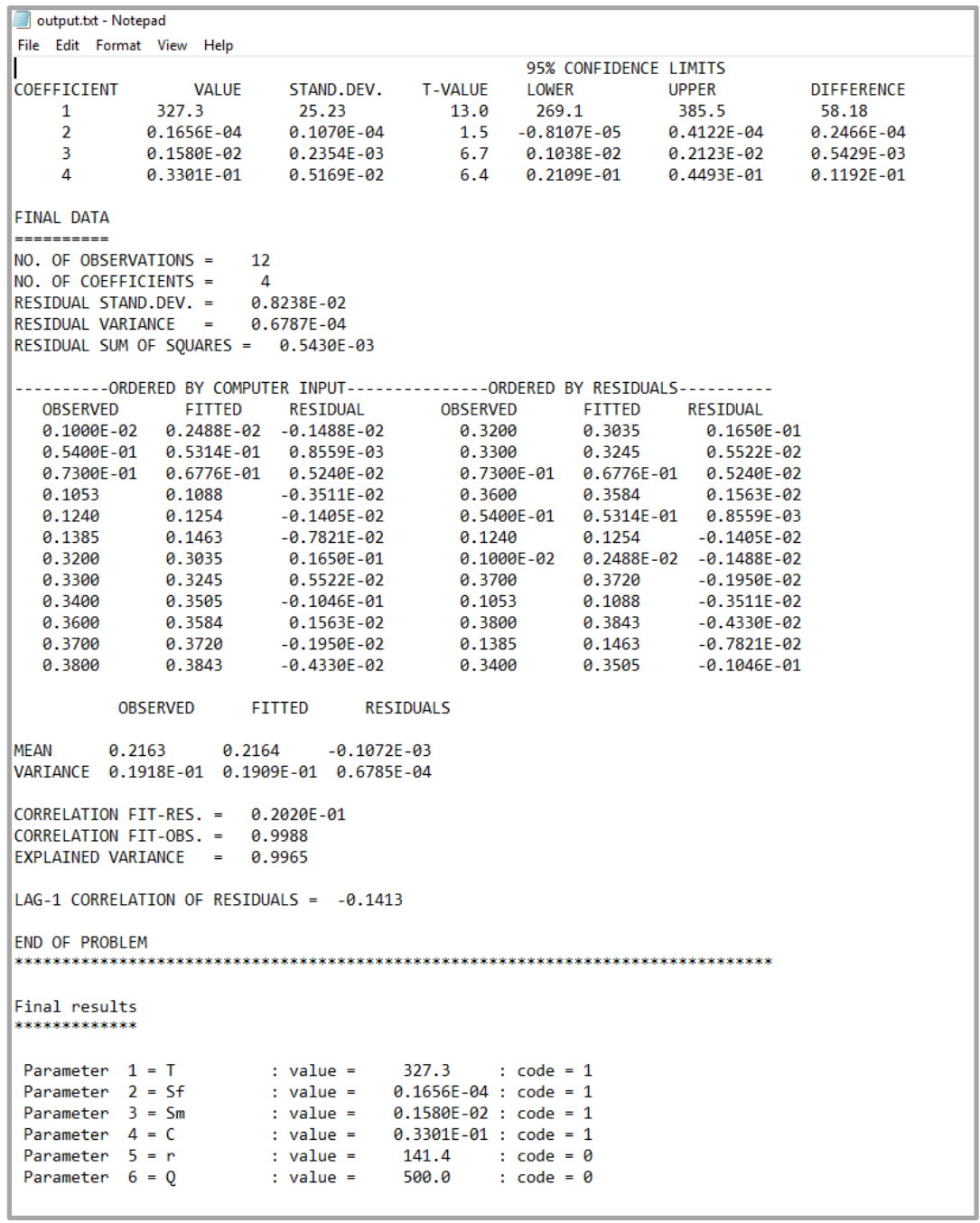

Figura 3.7: Ejemplo del archivo de resultados del programa de ajuste de De Smedt.

El procedimiento utilizado por De Smedt es un algoritmo de optimización basado en un método no lineal de mínimos cuadrados para justamente minimizar la suma de los residuos entre los valores observados y los calculados (Marquard, 1963). Es, en esencia, encontrar la mejor solución de la ec. (50) que es una integral impropia que debe ser evaluada por medio de métodos numéricos de integración y con una función de Bessel de primera especie y orden 0 en el integrando que es muy sesgada en sus límites; para llevar adelante esto De Smedt y Wierenga (1979) desarrollaron también una rutina que se encuentra contenida en el programa de optimización general. 
Lo más importante que se recomienda para que este programa encuentre buenos resultados es que los valores iniciales de los parámetros introducidos en el archivo de entrada sean lo más confiables posibles tanto desde su realidad física como de los trabajos previos de estimación que se puedan aportar. Esto se traduce entonces en otra clave para el desarrollo de la metodología que se propone en esta tesis, en especial, para tener valores de partida lo mejor estimados posibles para el coeficiente $\mathrm{C}$ de la FTMF.

En efecto, se ponen en juego 6 parámetros que definen el sistema de doble porosidad $(Q$, $r, T, S_{m}, S_{f}$ y C). Como ya se dijo, en el caso del caudal de bombeo (Q) y la distancia al pozo de observación ( $r$ ) se toman como datos ciertos del problema ya que los mismos son medidos y verificados con frecuencia en campo; de esta forma, quedan 4 parámetros para estimar con la técnica de optimización. El coeficiente $C$ de la FTMF es el que ofrece mayor incertidumbre, razón por la cual se propone en el siguiente punto un método para su valoración.

Para finalizar, en el Anexo 8.1 de este trabajo de tesis, se incluyen dos ejemplos de análisis de ensayos de bombeo con la técnica de De Smedt, donde se muestra la eficiencia de la rutina en casos reales de la práctica. En el primero de ellos, se reproduce el mismo ejemplo que da el autor en su trabajo: el sistema acuífero de Madison (Dakota del Sur, EE. UU.), reportado por Greene (1993); y siguiendo con el acuífero de Yuca Mountain (Nevada, EE. UU.) que fuera analizado por Moench (1984).

\subsubsection{Método combinado: secuencia a seguir, opciones y estimación de parámetros.}

En función de lo desarrollado hasta aquí, se resumen a continuación la secuencia de las operaciones a seguir con la propuesta metodológica que se introduce en esta tesis y las opciones que se promueven en relación directa con la información de partida disponible para estimar los parámetros hidrogeológicos desde un ensayo de bombeo.

En primer lugar, correspondería acudir a información de campo prexistente para tomar conocimiento de las características del sistema de fracturas reinante en la zona bajo estudio. Los datos básicos por recabar serían los relacionados con la orientación (azimut / rumbo, dirección e inclinación del buzamiento y rake/giros en el plano de falla) del sistema de fracturas.

En segundo lugar, la identificación del número de familias que integran el sistema de fracturas, en arreglo a disponer un modelo 1D, 2D o 3D de la representación estocástica. En general, en este punto puede afirmarse que las familias siempre están conjugadas de tal manera que se presentan en campo como sistemas ortogonales entre sí cuando las mismas son el resultado de plegamientos u otro tipo de fenómenos tectónicos. La aparición de diferentes familias de diaclasas o de líneas de disolución de la roca es mucho más caótico, razón por la cual es mucho más útil apuntar a su idealización como un sistema continuo macro-poroso donde la densidad de las pequeñas fisuras y su grado de conexión definen su conductividad intrínseca.

En tercer lugar, una idea aproximada de la proporción en volumen del sistema de fracturas con respecto al volumen total del acuífero. Esto puede inferirse a partir de testigos de perforación obrantes en los sitios de sondeo, en especial, si estos involucran a todo el espesor de la formación que se está investigando.

Una vez transitadas estas primeras actividades aparece entonces una primera opción para concretar el primer paso metodológico que consiste en la estimación del coeficiente de forma $\alpha$. Esta opción tiene que ver con el grado de definición alcanzado: si no se cuenta con información precisa de la orientación, número de familias o proporción de las fracturas, entonces se puede acudir a las expresiones (52), (53) y (54) para estimar $\alpha$ sólo adoptando el 
modelo dimensionalmente más plausible. El modelo 1D se encuentra muchas veces relacionado a acuíferos muy estratificados donde la doble porosidad se dispone por capas intercaladas. El sistema 2D y 3D son los más frecuentes dado que, como se adelantó, la porosidad secundaria responde a fracturas casi siempre ortogonales entre sí o muy cercanas a esa disposición con familias que siguen las direcciones principales de las tensiones mecánicas.

Si en cambio la información referida está disponible entonces la recomendación se perfila al uso de la herramienta SISIM y al programa de cálculo del factor de forma que lo obtiene como el cuadrado de la relación entre la superficie de intercaras y el volumen de referencia.

Una vez obtenido $\alpha$, el segundo gran paso es la estimación de los parámetros hidrogeológicos del acuífero naturalmente fracturado donde juega un rol fundamental el coeficiente C de la FTMF. Para eso se utiliza el modelo y la rutina de optimización de De Smedt ya comentada en el punto anterior.

Pero aquí aparece la segunda opción de procedimiento que consiste en emplear primero el coeficiente de forma $\alpha$, la conductividad de la interfase $K_{i}$ (normalmente asimilada a la $K_{m}$ de la matriz) y el espesor de la formación para computar C de la FTMF tal cual se lo define con la ecuación (8). Como alternativa en este punto se puede recurrir primero al método de De Smedt para optimizar el valor de $\mathrm{C}$ y luego deducir la conductividad $\mathrm{K}_{\mathrm{i}}$ dividiendo ese coeficiente por el producto de $\alpha$ por $b$ (espesor del acuífero confinado). Este último camino es el que se recomienda en este trabajo, reforzando el concepto tan aludido aquí de no asimilar automáticamente como cierta la igualdad $\mathrm{K}_{\mathrm{i}}=\mathrm{K}_{\mathrm{m}}$, reservando el valor del primer miembro de esa igualdad a la importancia que tiene el poder reportar un valor intrínseco y característico de la transición matriz-fisura.

Para resumir lo expuesto, se presente el siguiente diagrama conceptual del método propuesto:

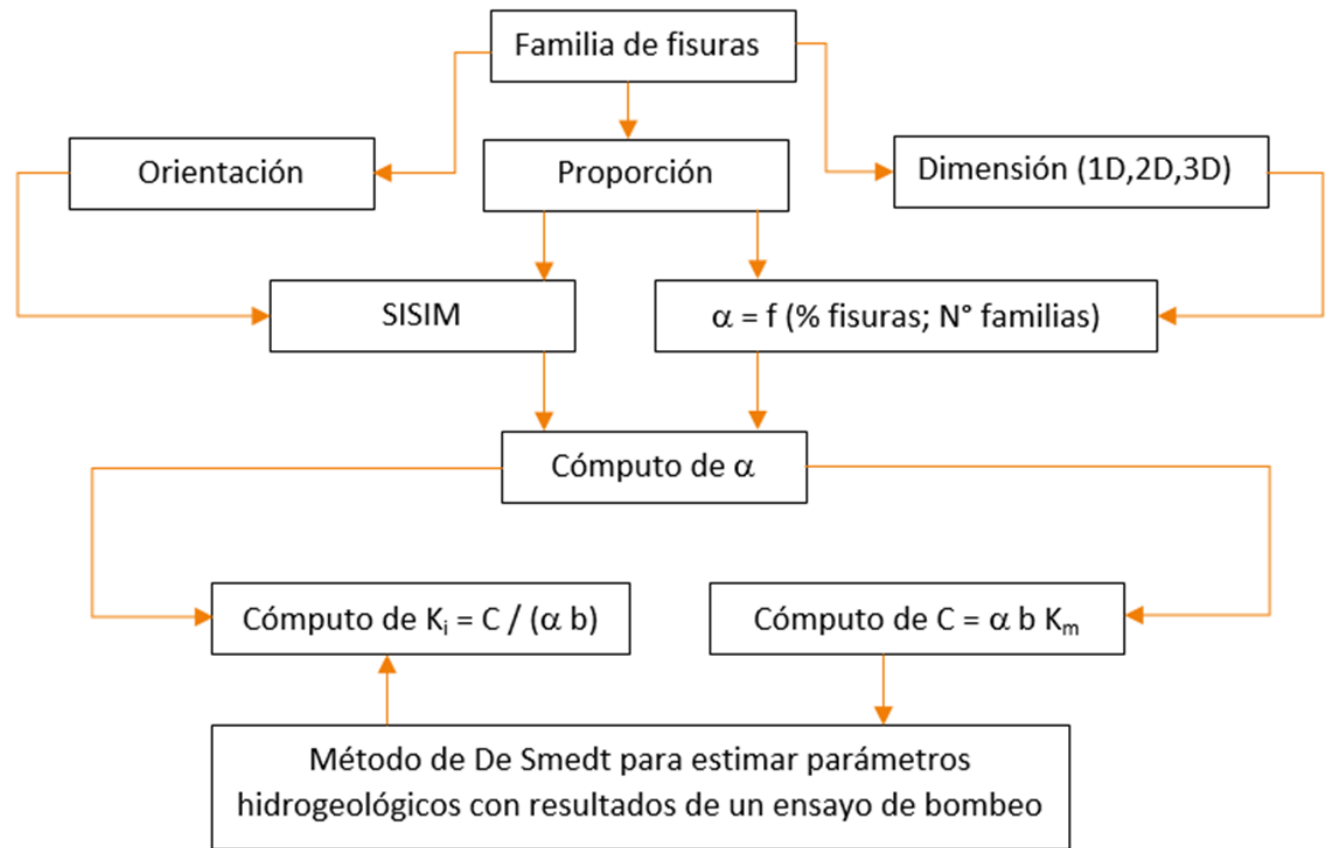

Figura 3.8: Diagrama de flujo del procedimiento propuesto. 


\subsubsection{Ajuste de acuerdo con el tipo de régimen desarrollado en la interface matriz-fisura}

Como se describiera en el punto 2.1.3 es importante diferenciar el régimen que se asimila mejor al comportamiento hidrodinámico de la respuesta de la FTMF, referido allí como modelos cuasi-estacionario (o lineal) y modelo no lineal de cesión de flujo desde la matriz al sistema de fisuras.

Afortunadamente, el modelo De Smedt tiene la ductilidad suficiente como para representar con buena aproximación el modelo de Barenblatt y al mismo tiempo modelos más complejos como el de drenaje diferido o el modelo de Boulton, todos ellos ya citados con anterioridad.

En efecto, como se verá más adelante al considerar los casos sintéticos para testear la metodología propuesta en esta tesis, básicamente el modelo de De Smedt puede asimilarse a ambos casos con suficiente aproximación, en especial, para tiempos prolongados de bombeo. En los primeros instantes del bombeo, la rigidez del modelo cuasi-estacionario no puede reproducir la rapidez de cambio de la cesión de volumen de agua desde la matriz y se retrasa con respecto al modelo no lineal.

Como ya se dijo, si los bloques que representan a la matriz son muy extensos y su permeabilidad es relativamente baja entonces los mismos funcionan como un recipiente a presión donde la caída de presión se produce en forma abrupta y en forma lineal al alcanzar el sistema de fisuras. Si se permite la analogía con los escurrimientos a presión en tuberías, el modelo lineal se comporta más como una pérdida local en una línea producida por ejemplo por una válvula o un estrechamiento. En cambio, para el modelo no lineal la analogía sugiere que la pérdida de carga se produce como una resistencia general que involucra a todo el sistema matricial donde la inclinación de la línea de energía piezométrica se propaga. 


\section{RESULTADOS DE LA APLICACIÓN DEL MÉTODO PROPUESTO}

\subsection{VERIFICACIÓN EN CASOS SINTÉTICOS CON SOLUCIÓN ANALÍTICA CONOCIDA}

Una vez simuladas las familias de fracturas y calculado el factor de forma correspondería aplicar este último en la FTMF para, según el caso a analizar, mejorar la estimación de los parámetros de los ensayos de bombeo en acuíferos fracturados. Antes de aplicar el método a un caso real, se verificó este paso en casos sintéticos con solución analítica conocida.

\subsubsection{Modelo cuasi-estacionario de interacción matriz-fisura en acuífero confinado (caso A).}

El ejemplo sintético se tomó del trabajo de Huyakorn et al. (1983) y se modificó para trabajar con los siguientes datos de partida:

- $\quad Q$ (caudal de bombeo) $=250 \mathrm{~m}^{3} /$ día

- $\quad T_{\mathrm{f}}$ (Trasmisividad de las fisuras) $=10 \mathrm{~m}^{2} /$ día

- $\quad S_{m}$ (coeficiente de almacenamiento de la matriz) $=0,02[-]$

- $\quad \mathrm{S}_{\mathrm{f}}$ (coeficiente de almacenamiento de las fisuras) $=0,002[-]$

- $\quad$ C (coeficiente de la FTMF) $=0,0002=2 \times 10^{-4}$ día $^{-1}$

Los pozos de observación se ubican a $r_{1}=45 \mathrm{~m}$ y a $r_{2}=100 \mathrm{~m}$ del pozo de bombeo. Se tienen toda la información para usar la función de pozo de De Smedt $\left(W_{2}\right)$ por lo que queda sólo comparar los descensos producidos con esta fórmula con los que resultan de la solución analítica. Para sintetizar esa comparación, se volcaron los resultados en la siguiente figura:

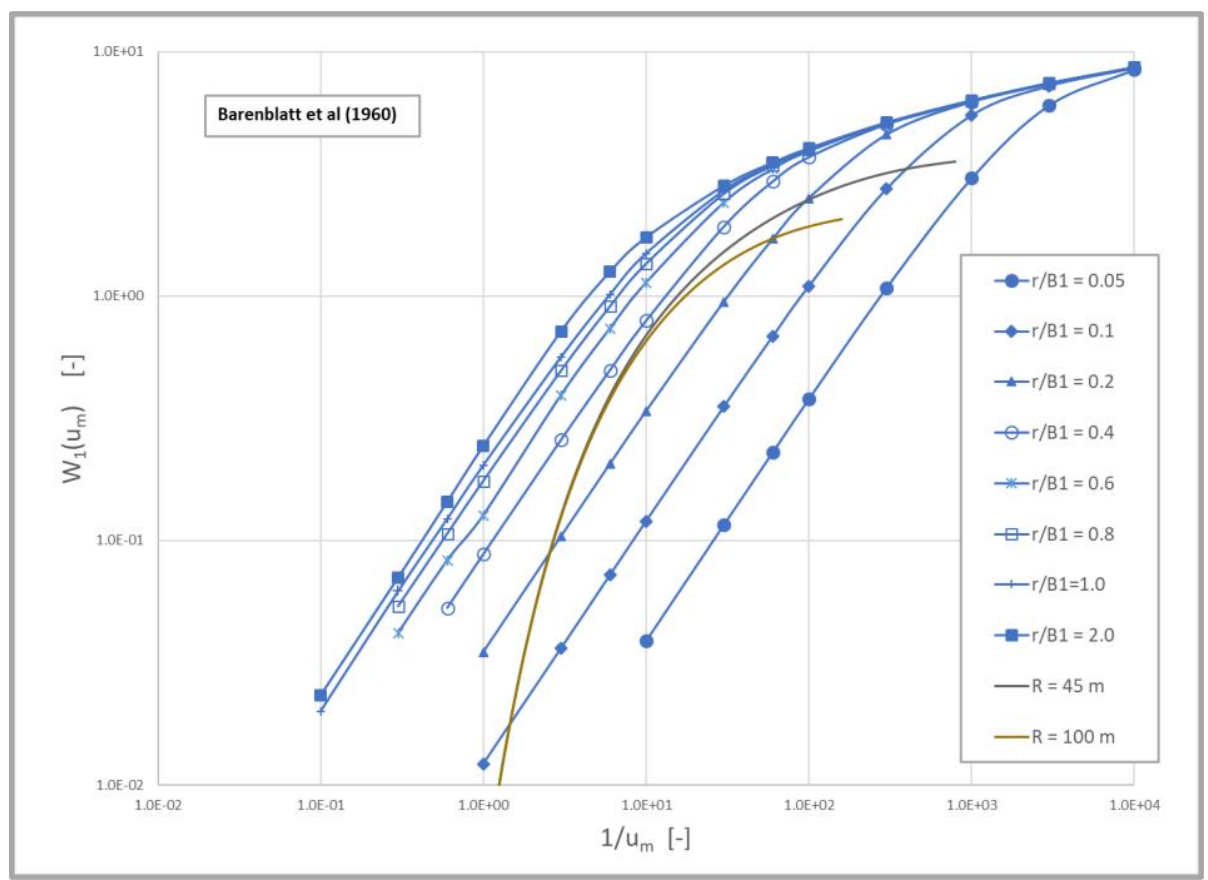

Figura 4.1: Comparación de descensos (solución analítica vs. $W_{2 p}$ ). 
Como se ha anticipado en el capítulo anterior, el modelo de Barenblatt et al. (1960) es mucho más rígido a la hora de ceder agua desde la matriz en comparación con el modelo de De Smedt ya citado. Se puede apreciar cómo este último comienza declinar la pendiente de la curva del descenso mucho antes, lo cual denuncia un aporte de agua adicional de agua al sistema, en este caso proveniente de la matriz porosa.

\subsubsection{Acuífero confinado con fracturas planas horizontales (caso B)}

El esquema de este caso es similar al anterior salvo que en esta oportunidad se consideran dos fracturas horizontales planas ubicadas en forma simétrica en una altura de $30 \mathrm{~m}$ del bloque. Los espesores de las fisuras son despreciables y la base horizontal del bloque es de $1000 \mathrm{~m} \mathrm{x}$ $1000 \mathrm{~m}$. El caudal de explotación, las distancias a los pozos de observación, la trasmisividad de las fracturas y la relación de coeficientes de almacenamiento son similares al caso (sólo Sm se tomó igual a 0.018 para que coincida con la curva tipo donde $\eta=10$ ).

Se agrega aquí una conductividad de la matriz $\mathrm{K}_{\mathrm{m}}=5 \times 10^{-3} \mathrm{~m} /$ día, razón por la cual es ahora posible calcular el coeficiente $C[1 /$ día $]=\alpha \mathrm{K}_{i} b \approx \alpha \mathrm{K}_{\mathrm{m}} \mathrm{b}$; con $\mathrm{b}=30 \mathrm{~m} ; \mathrm{K}_{\mathrm{m}}=0,005 \mathrm{~m} /$ día y $\alpha=(4 \times 1000 \times 1000) /(1000 \times 1000 \times 30)=0,133 \mathrm{~m}^{-2}$ (son 4 intercaras) se tiene $C=0,02$ día $^{-1}$. Introduciendo estos valores en la solución de De Smedt podemos graficar las curvas tipo para este caso.

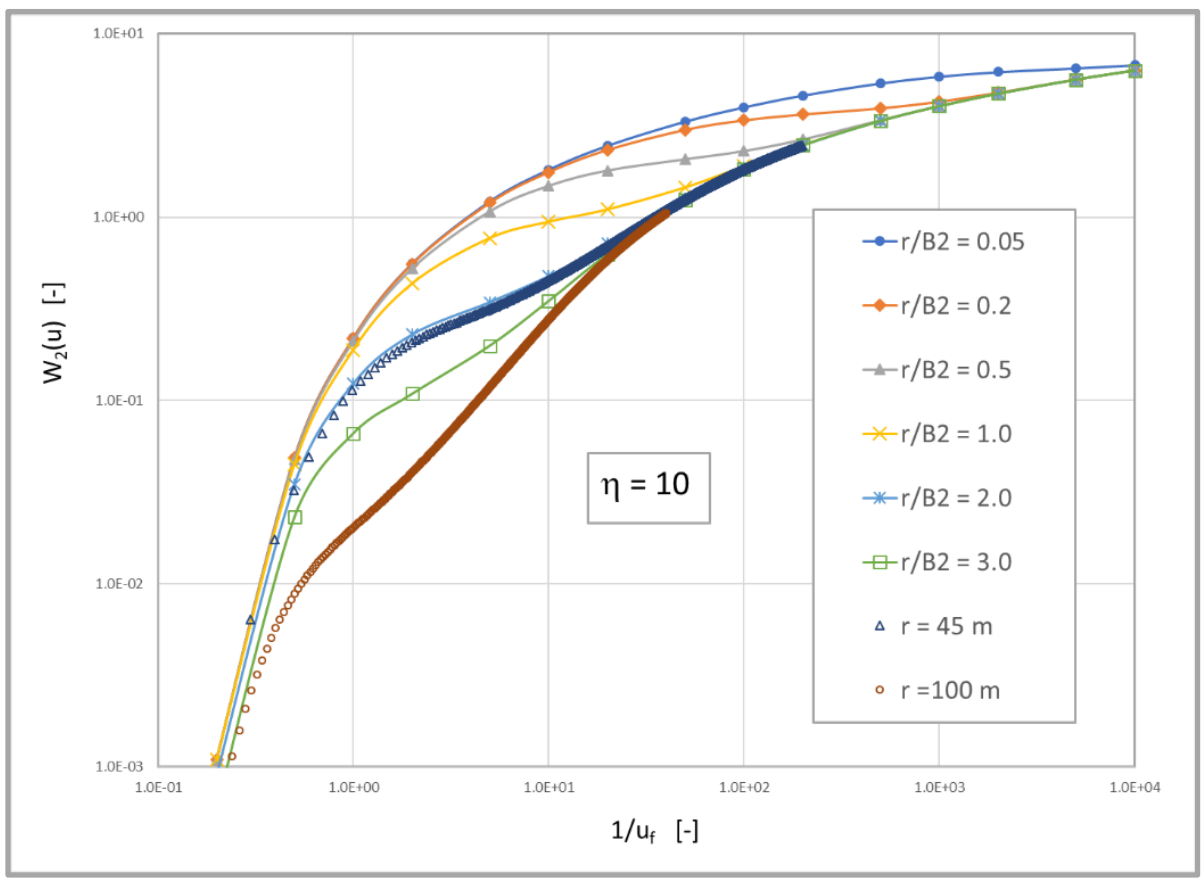

Figura 4.2: Comparación de descensos Boulton vs. De Smedt.

En la figura anterior se puede apreciar claramente la coincidencia del modelo de Boulton con el modelo de De Smedt para el radio $r=45 \mathrm{~m}$ (marcas triangulares) y como un simple cómputo de $\mathrm{C}$ a través de un sencillo cálculo del factor de forma puede ser reproducido con esta metodología. Se debe destacar que los puntos correspondientes al pozo más alejado $(r=100 \mathrm{~m}$ ) corresponde a una relación $\mathrm{r} / \mathrm{B} 2=5.0$ por lo que la comparación se hace sobre la gráfica a efectos de destacar el comportamiento típico de drenaje diferido, en este caso, el aporte de la matriz al sistema de fisuras pero se aclara que no ha sido volcada en el gráfico la curva tipo por no disponer de su valores tabulados. 


\subsubsection{Fractura vertical en acuífero confinado (caso C)}

Usamos nuevamente el esquema del caso A pero ahora se agrega una fractura vertical con el pozo totalmente penetrante en ella. Con las mismas dimensiones de bloque que en el caso $B$, ahora $\alpha=(2 \times 30 \times 1000) /(1000 \times 1000 \times 30)=0,002 \mathrm{~m}^{-2}$ y C $=0,005$ (dado que ahora $b$ $=500 \mathrm{~m}$ ). La solución con la ecuación de pozo $\mathrm{W}_{2 \mathrm{P}}$ (De Smedt, 2011) se presenta en la siguiente figura:

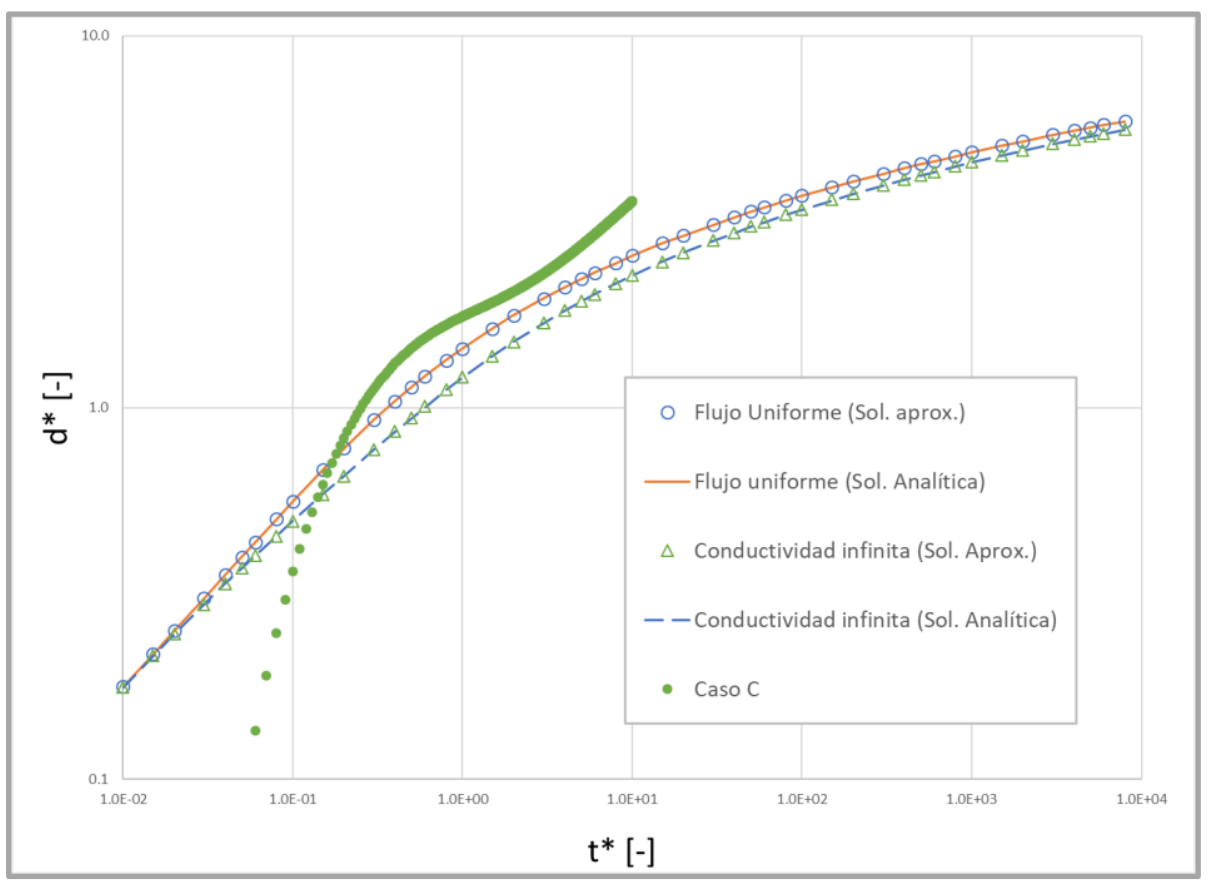

Figura 4.3: Comparación de descensos en los pozos de observación para el caso C.

Se puede observar cómo un modelo tan diferente al caso de la fractura vertical aún es útil para reproducir en el espacio descensos-tiempo adimensional los valores que se obtienen de la solución analítica.

También es necesario aclarar que en la figura 4.3 sólo se representaron las curvas límites para los descensos en el pozo central de bombeo y que necesariamente la curva del caso representada (que corresponde a $r=45 \mathrm{~m}$ ) debe desplazarse hacia arriba para tener en cuenta el desplazamiento del pozo de observación. La curva tipo y la gráfica correspondiente a valores de los descensos para puntos de la matriz alejados de la fractura vertical pueden ser consultados en el trabajo original de Gringarten (1974).

\subsection{Aplicación a la formación acuífera del distrito "Cerro Solo", Chubut, Argentina}

El objetivo de este trabajo fue la caracterización hidrodinámica regional de una formación acuífera cretácica perteneciente al Grupo Chubut en la provincia homónima de la República Argentina (Fig. 4.4 y 4.5 ).

En el Departamento Paso de Indios de esa provincia, este grupo se reconoce localmente como la formación Los Adobes (Bianchi, 1995) y está compuesto por dos miembros: Arroyo del Pajarito (conglomerado con matriz de gravas y arenas, areniscas; ver afloramiento en Foto 4.1) y Bardas Coloradas (limolitas, arcilitas tobáceas y tobas). 


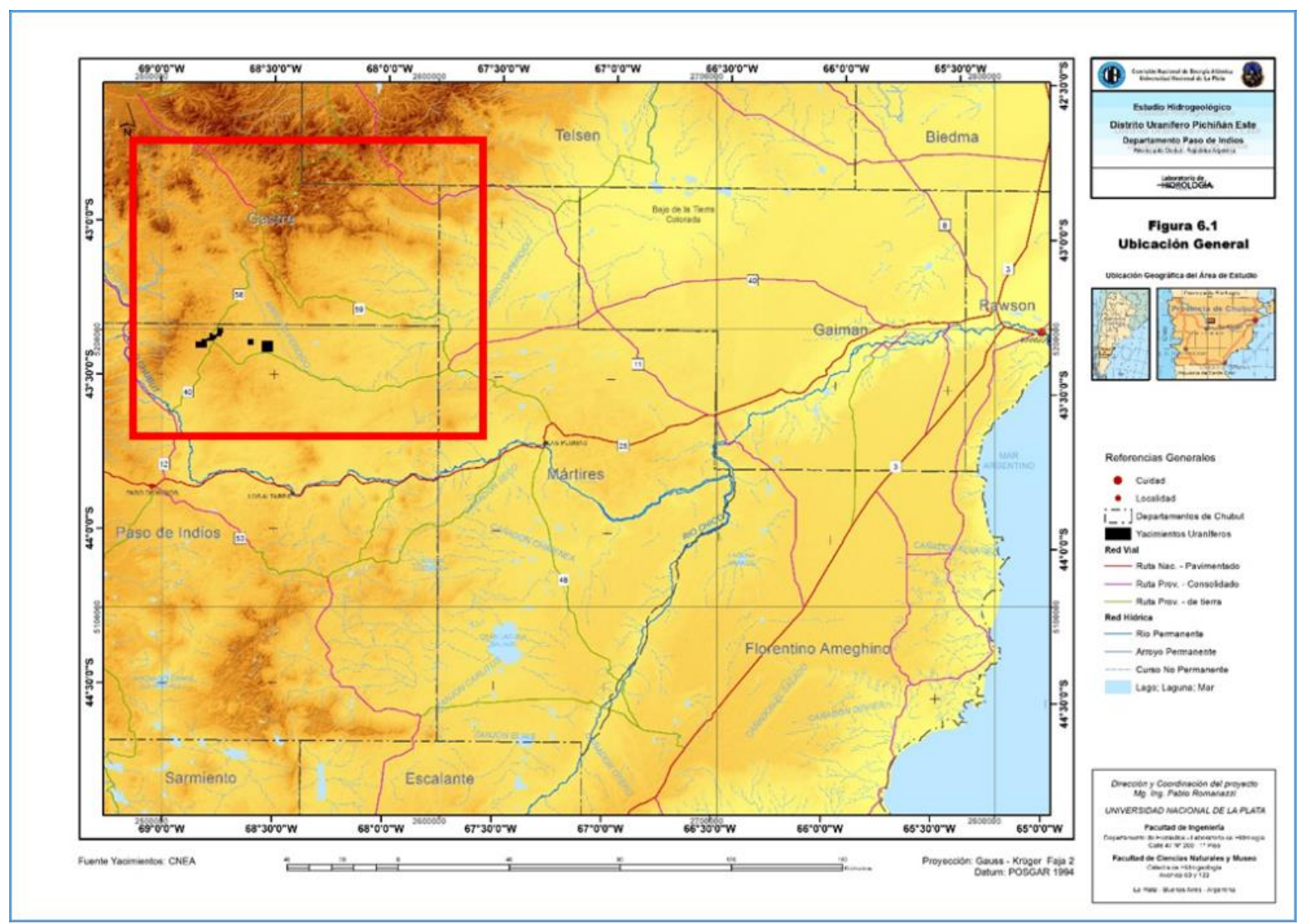

Figura 4.4: Ubicación de la región bajo estudio.

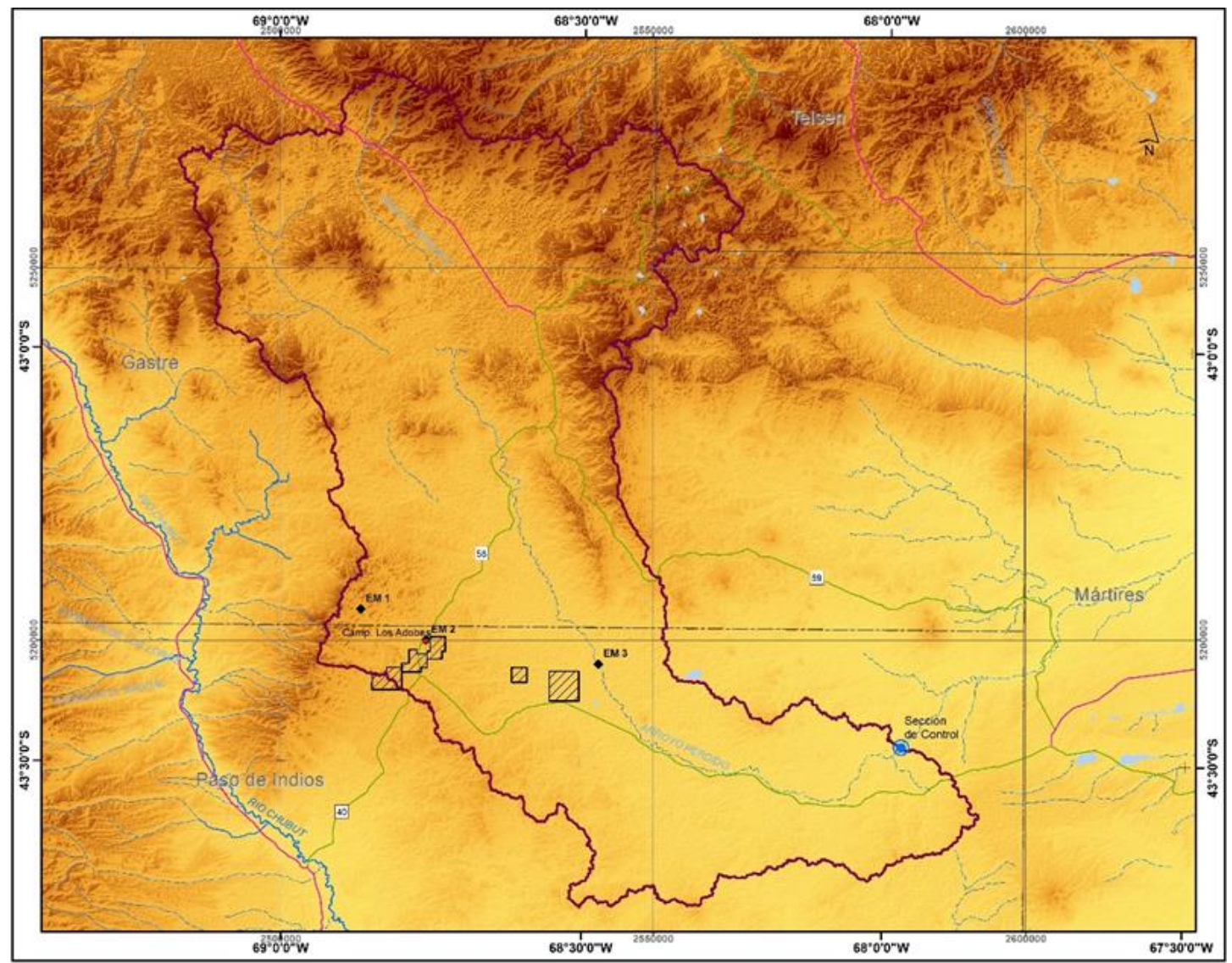

Figura 4.5: Ubicación de los sitios ensayados en la cuenca del arroyo Perdido. 


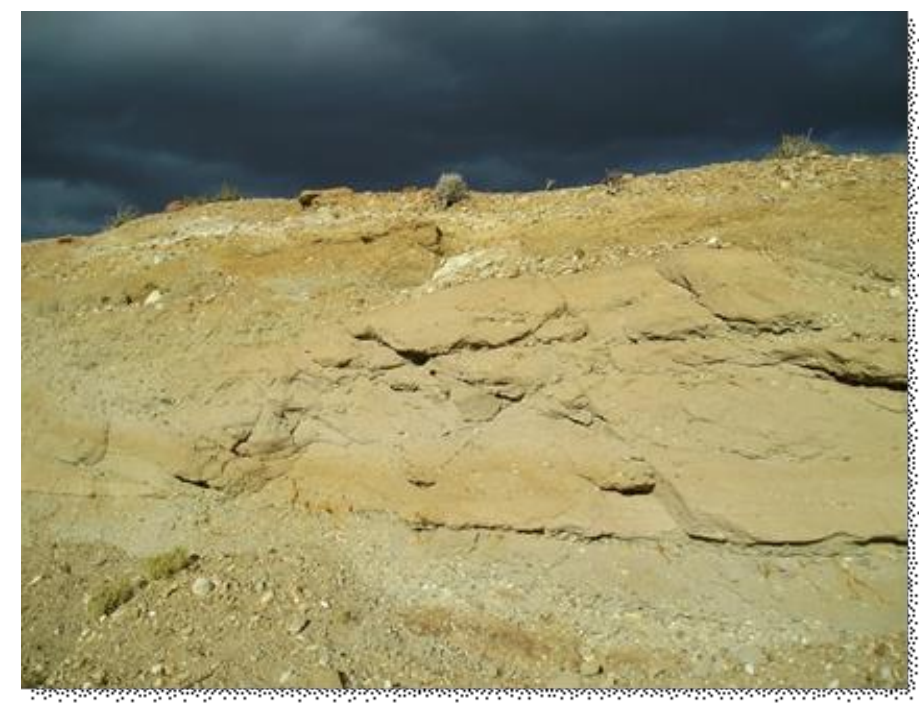

Foto 4.1: Afloramiento en superficie del Mo. Arroyo del Pajarito.

\subsubsection{Características hidrogeológicas de la región}

Desde el punto de vista hidrogeológico (Hernández et al., 2012) existen tres unidades reconocibles en toda la región: (i) una unidad post-cretácica que incluye a un acuífero freático de reducido espesor; (ii) una unidad cretácica constituida por el miembro Bardas Coloradas de comportamiento acuícludo a acuitardo que suprayace al miembro inferior Arroyo del Pajarito, principal acuífero de la zona de tipo confinado y caracterizado por poseer doble porosidad (primaria intergranular y secundaria por fisuración); y (iii) una unidad jurásica inferior, de carácter acuífugo, que constituye el hidroapoyo del sistema.

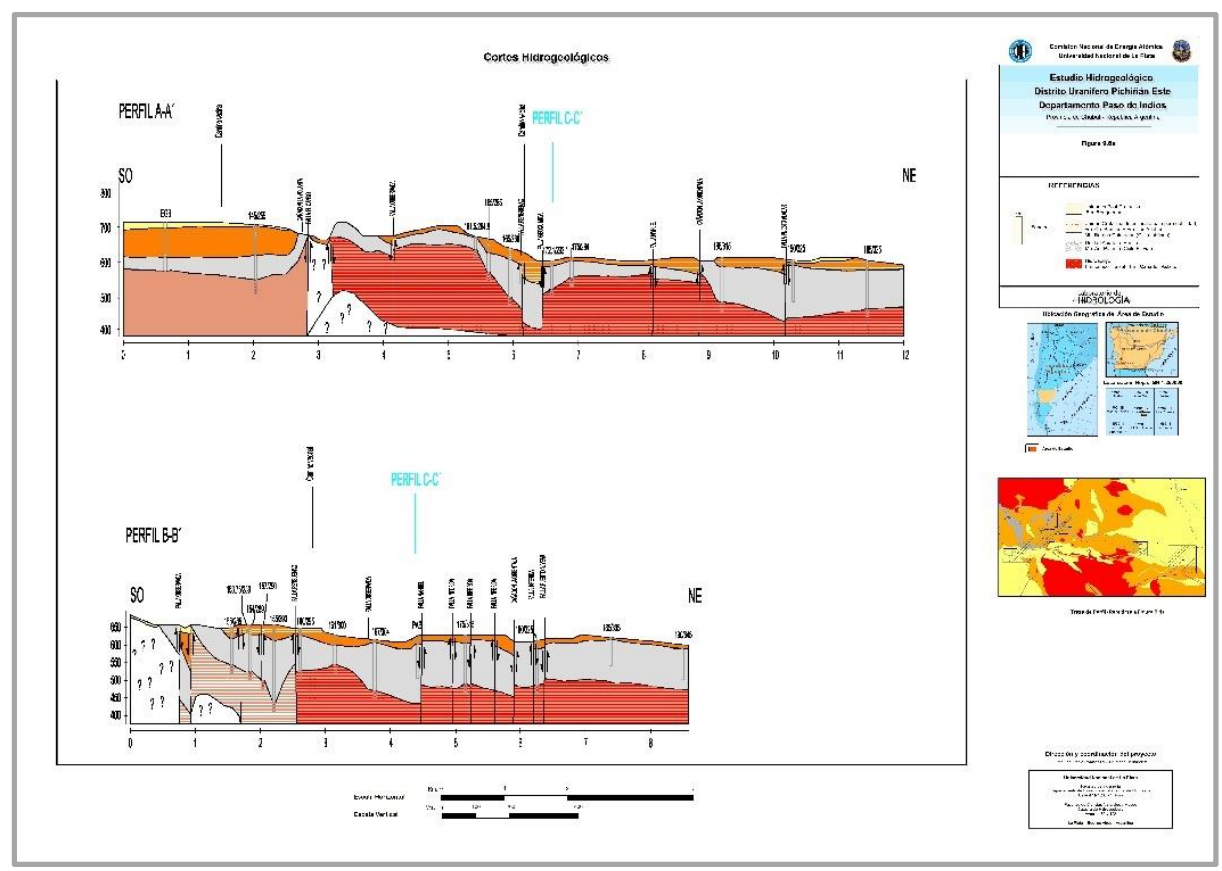

Figura 4.6: Corte geológico AA y BB de la formación (fuente: Hernández et al., 2012). 


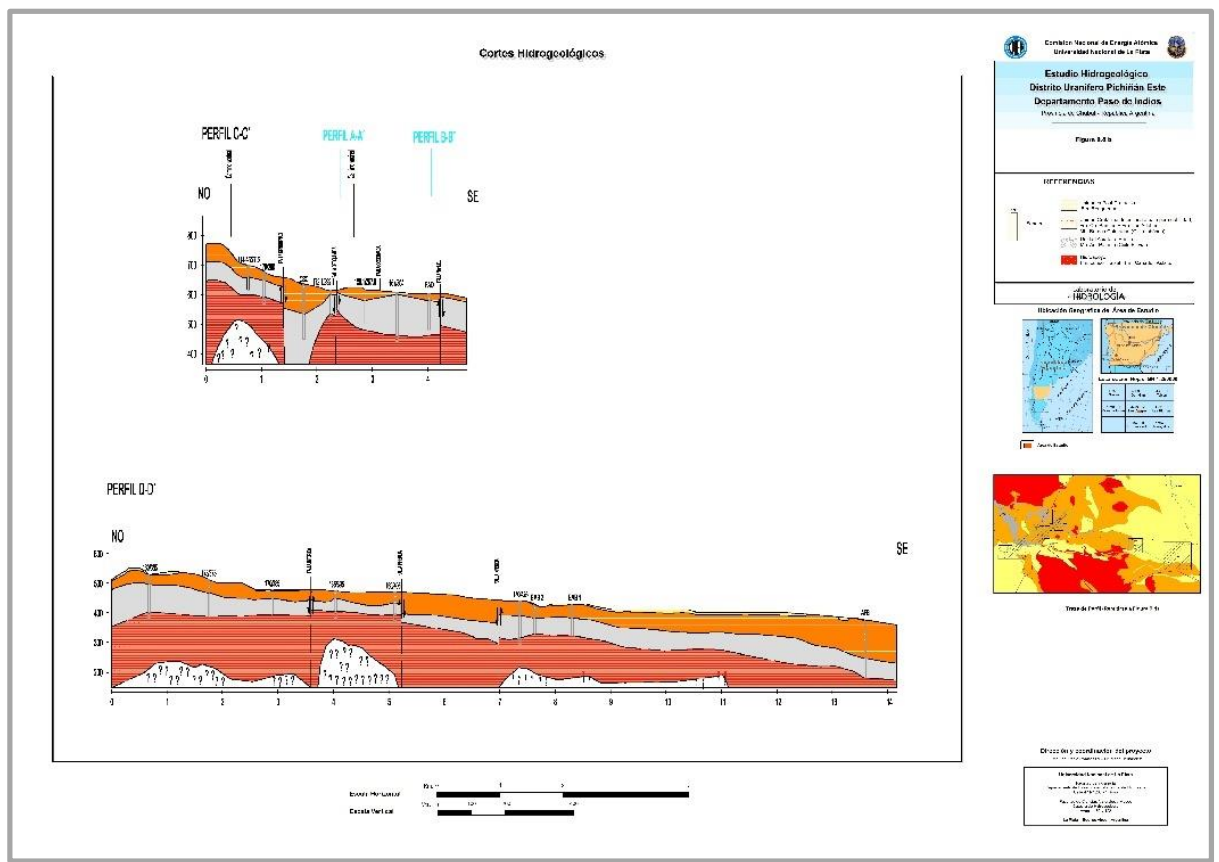

Figura 4.7: Corte geológico CC y DD de la formación (fuente: Hernández et al., 2012).

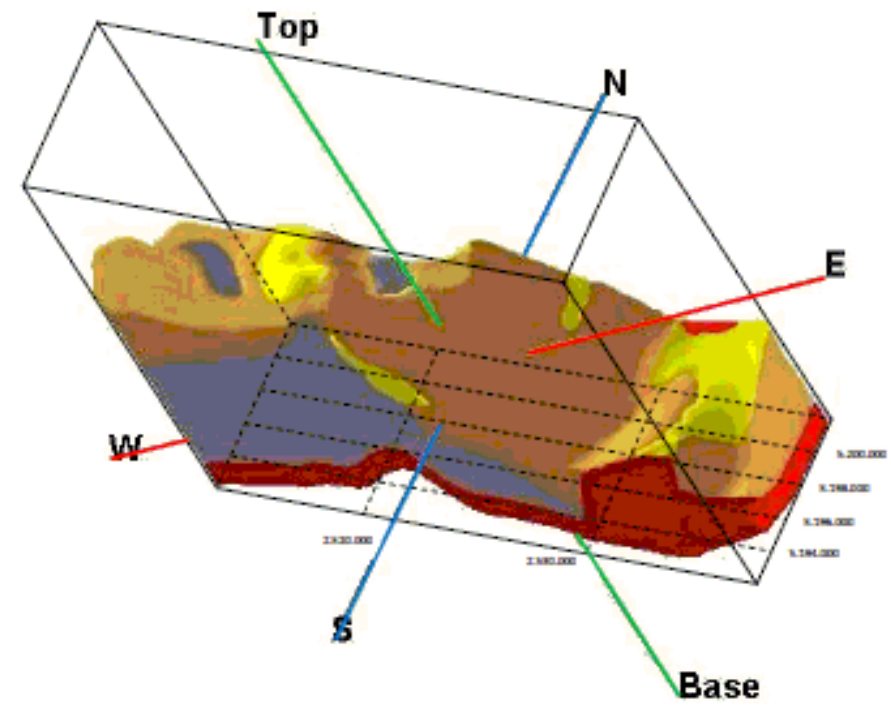

Referencias

Unidad post Cretácica

Unidad Cretácica scuifera

Unidad Cretácica de escasa permeabilidad

Hidrospoyo

Figura 4.8: Corte geológico 3D de la formación (fuente: Hernández et al., 2012). 
Desde el punto de vista hidrodinámico, el flujo en el acuífero circula de oeste a este, con recarga alóctona subterránea y un reducido aporte directo donde este acuífero aflora en superficie - comportándose como libre -, mientras que en el tramo medio y en la descarga se comporta como confinado siguiendo el eje del valle del arroyo Perdido, cuenca endorreica con nacientes en la Sierra del Pichiñán (Fig. 4.5).

Los parámetros hidrogeológicos de la región bajo estudio fueron evaluados mediante ensayos de bombeo (Hernández et al., 2012) en la zona de pedemonte (yacimientos Cerro Solo, Puesto Alvear y El Ganso) y en la Ilanura baja (yacimientos El Molino y Arroyo Perdido), acompañada de registros piezométricos y freáticos de una red de más de 60 perforaciones que se monitorea desde el año 2007 a la fecha.

Estos ensayos sirvieron para estimar la conductividad hidráulica $\mathrm{K}[\mathrm{L} / \mathrm{T}]$ del medio acuífero confinado (rango más frecuente entre $1 \times 10^{-1}$ a $1 \times 10^{0} \mathrm{~m} / \mathrm{d}$ en la zona alta occidental y variación entre $1 \times 10^{-2}$ a $1 \times 10^{-3} \mathrm{~m} / \mathrm{d}$ en la zona baja oriental de la cuenca) así como también del coeficiente de almacenamiento $\mathrm{S}[-]$ (rango comprendido entre $10^{-4}$ a $10^{-6}$ ); este último, con un rol principal en el cálculo de las reservas subterráneas en la zona.

\subsubsection{Programa de ensayos de bombeo y resultados obtenidos}

El programa de ensayos de bombeo (ver Anexo 8.2 de esta tesis) incluyó una primera prueba breve a caudal variable, cuya finalidad fue estimar los caudales de bombeo más adecuados y las depresiones de nivel esperables en función de los descensos registrados. Se realizó luego una segunda prueba de larga duración a caudal constante y recuperación, siempre registrando niveles en pozos de observación existentes y otros (la mayoría) construidos ad-hoc (Hernández et al., 2012).

En este trabajo se han reinterpretado estos ensayos utilizando la solución analítica desarrollada por De Smedt (2011) para flujo radial afluente a un pozo con bombeo $Q\left[\mathrm{~L}^{3} \mathrm{~T}^{-1}\right]$ en un medio naturalmente fracturado, ya presentada en el capítulo 2 de esta tesis.

La anisotropía y heterogeneidad primaria de la unidad cretácica acuífera estudiada y la doble porosidad aportada por fisuras, dificulta la obtención de una regionalización precisa de los parámetros hidrogeológicos (Hernández et al., 2012).

No obstante, al incorporar explícitamente los parámetros de la función de transferencia expresada por la ecuación que se define como función de transferencia, se puede avanzar en una caracterización más coherente.

La aplicación de la metodología descripta a los resultados de los ensayos de bombeo disponibles, permitió mejorar la estimación de los parámetros tradicionales ( $T, \mathrm{~K}$ y S) así como también adicionar un conocimiento más aproximado acerca de la influencia de la doble porosidad en la dinámica subterránea.

Además de tener en cuenta que existe una disminución verificada de la capacidad de conducción de oeste a este (probablemente debido a la presencia de texturas más finas en la componente matricial del sector oriental del paleocauce Arroyo del Pajarito), se han obtenido buenos resultados en la calibración del modelo hidrodinámico regional (Hernández et al., 2012) reduciendo en un orden de magnitud los valores regionales del coeficiente de almacenamiento (S). Esta práctica empírica permite aproximar en forma eficiente la influencia de la doble porosidad en las extracciones a largo plazo, toda vez que se concreta el aporte continuo de agua de la componente matricial en lugar del aporte temprano del volumen alojado en las fracturas. 
Con esta idea básica se procedió entonces a asumir que los valores iniciales de los parámetros $\mathrm{T}, \mathrm{K}$ y $\mathrm{S}$ son los obtenidos mediante los ensayos de bombeo (atribuibles al comportamiento temprano de la red de fisuras); a aproximar el coeficiente $S_{m}$ en un orden de magnitud inferior al anterior; $y$ a estimar el coeficiente de la función de transferencia (C) con un factor de forma $(\alpha)$ que resulta de un proceso iterativo hasta conseguir un ajuste aceptable con los descensos registrados en cada pozo de observación.

\subsubsection{Aplicación del método combinado propuesto}

En los yacimientos donde se realizaron ensayos de bombeo (Cerro Solo y Puesto Alvear, en la cuenca alta; El Molino y Arroyo Perdido, en la cuenca baja) se aplicó la rutina de ajuste desarrollada por De Smedt (2011). Los valores adoptados inicialmente son los interpretados en forma clásica por el método de Theis para luego aplicar la rutina de optimización con $Q$ y $r$ constantes (como es habitual debido a su baja incertidumbre) y liberando el algoritmo de búsqueda para el resto de los parámetros $\left(T, \mathrm{~S}_{\mathrm{m}}, \mathrm{S}_{\mathrm{f}} \mathrm{y} \mathrm{C}\right)$.

Los resultados obtenidos se consignan en la Tabla 4.1 en el sentido del flujo. En su última fila se vuelcan también los valores regionales que se estimaron mediante la modelización matemática del área (Hernández et al., 2012) que, como ya se adelantó, su calibración fue realizada en base a la piezometría disponible (período 2007 - 2012). En el Anexo 8.2 se incluye un resumen del trabajo citado y se aportan datos de los ensayos de bombeo efectuados.

Tabla 4.1: Estimación de parámetros mediante la solución analítica de De Smedt (2011)

\begin{tabular}{|l|cc|cc|}
\hline Sitio ensayado & $\begin{array}{c}K_{f} \\
{[\mathrm{~m} / \mathrm{d}]}\end{array}$ & $\begin{array}{c}K_{m} \\
{[\mathrm{~m} / \mathrm{d}]}\end{array}$ & $\begin{array}{c}S_{f} \\
{[-]}\end{array}$ & $\begin{array}{c}S_{m} \\
{[-]}\end{array}$ \\
\hline Cerro Solo & $4,8.10^{0}$ & $2,9.10^{-1}$ & $1,5.10^{-3}$ & $1,8.10^{-2}$ \\
Puesto Alvear & $5,2.10^{-1}$ & $1,8.10^{-2}$ & $1,8.10^{-4}$ & $1,6.10^{-1}$ \\
El Molino & $2,3.10^{-2}$ & $1,6.10^{-3}$ & $3,2.10^{-4}$ & $1,0.10^{-2}$ \\
Arroyo Perdido & $1,2.10^{-3}$ & $2,2.10^{-4}$ & $5,3.10^{-5}$ & $4,6.10^{-4}$ \\
\hline \multirow{2}{*}{ Valores regionales } & \multicolumn{2}{|c|}{1 a $10^{-1}$ (a. arr.) } & \multicolumn{2}{|c|}{$10^{-4}$ a $10^{-6}$} \\
\hline
\end{tabular}

El ajuste o nueva interpretación de los resultados de los ensayos de campo resultó ser aceptable, tal cual se muestra en la Figura 4.9.

En esta representación, la estimación inicial de la $W_{2 D P}$ se obtiene con los parámetros que surgen del método de Theis para un piezómetro ubicado a $25 \mathrm{~m}$ del pozo de bombeo (el ensayo se llevó a cabo durante 1 día completo manteniendo un régimen permanente a razón de $18 \mathrm{~m}^{3} / \mathrm{h}$ ). La curva ajustada posteriormente acompaña mejor a los datos experimentales y de su definición surgen los valores apuntados en la Tabla 4.1. Los resultados para otros sitios se presentan en las Figuras 4.10 a 4.12

En estos últimos casos, las distancias a los pozos de bombeo fueron similares al primer caso (entre 15 y $30 \mathrm{~m}$ ) y en todos se insinúa la transición desde el aporte temprano de la red de fisuras hasta la estabilización de la cesión de agua de la componente primaria, en especial, cuando los ensayos se han podido prolongar lo suficiente como es el caso del ensayo realizado en el Yacimiento Puesto Alvear (Fig. 4.10). 


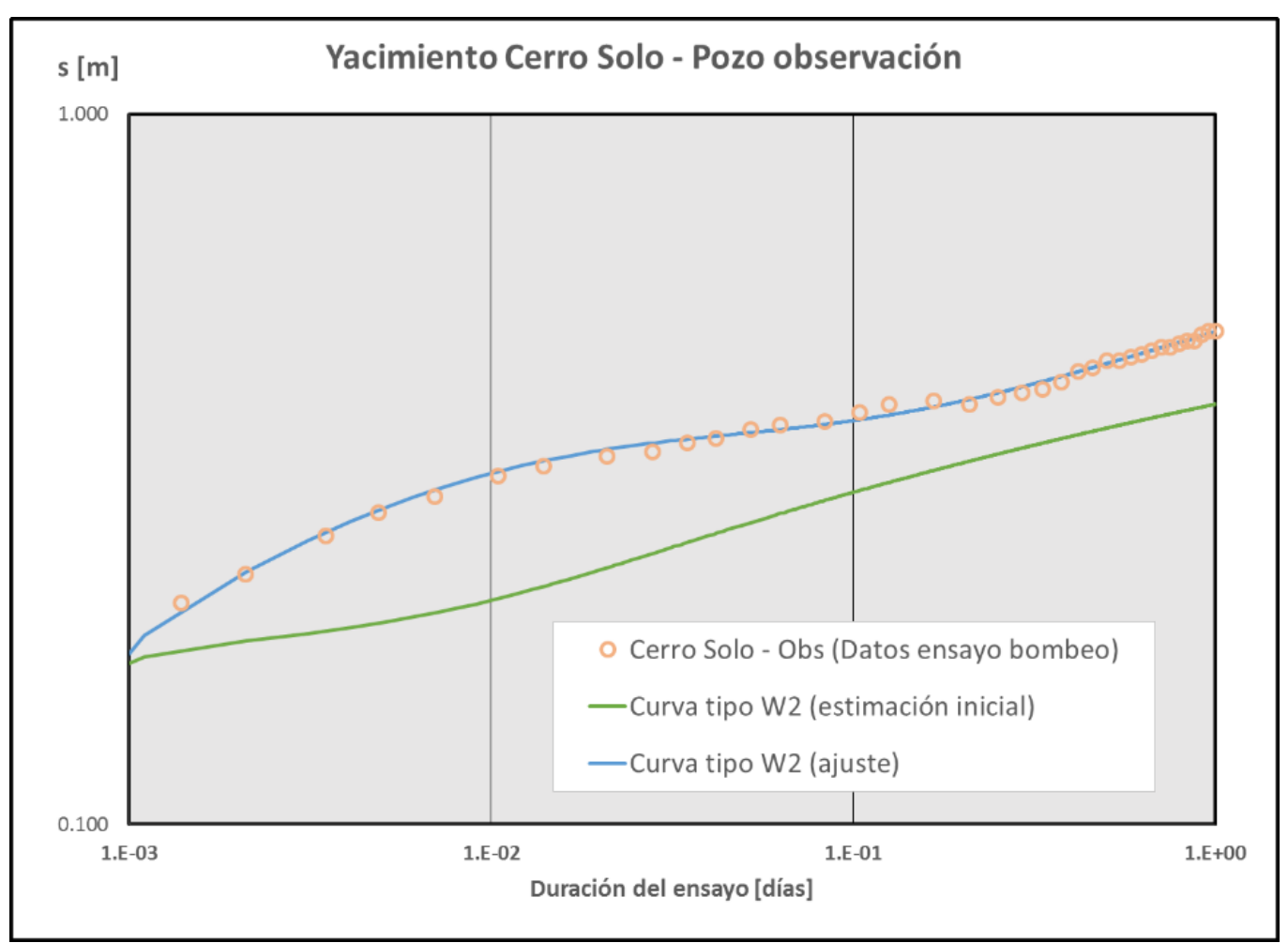

Figura 4.9: Datos del Ensayo de bombeo y ajuste de la W2DP en el sitio Cerro Solo.

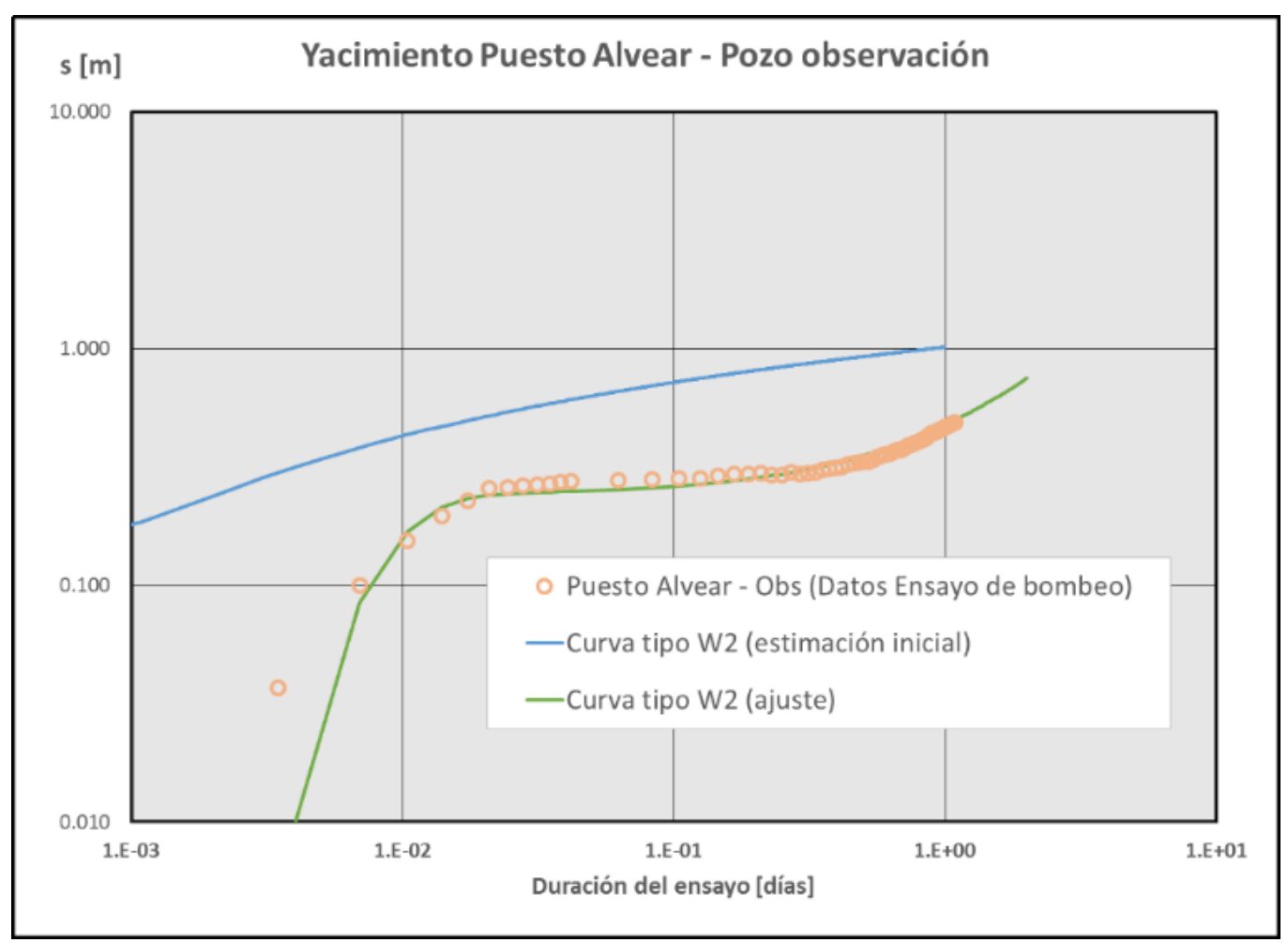

Figura 4.10: Datos del Ensayo de bombeo y ajuste de la W2DP en el sitio Puesto Alvear. 


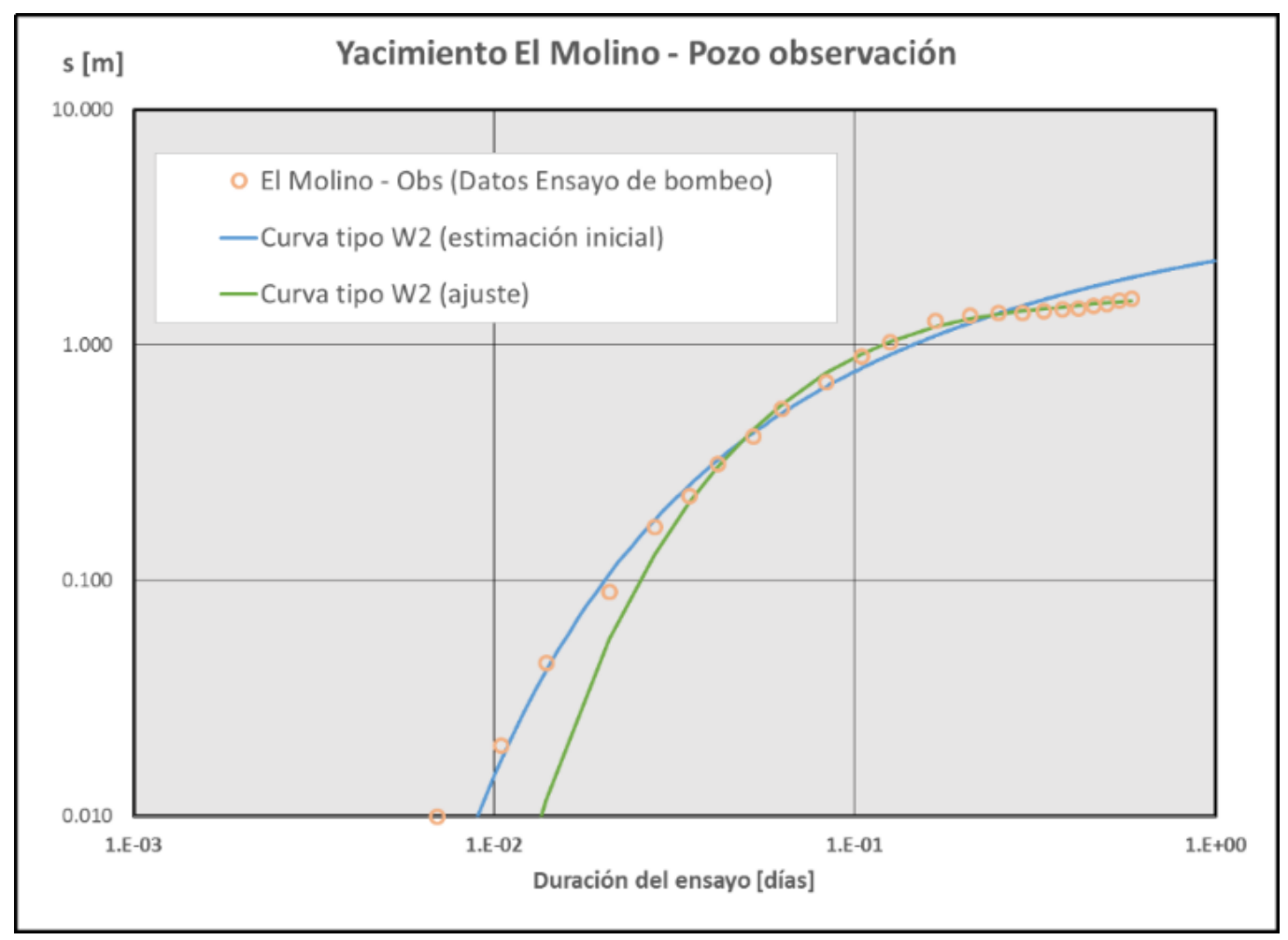

Figura 4.11: Datos del Ensayo de bombeo y ajuste de la W2DP en el sitio El Molino.

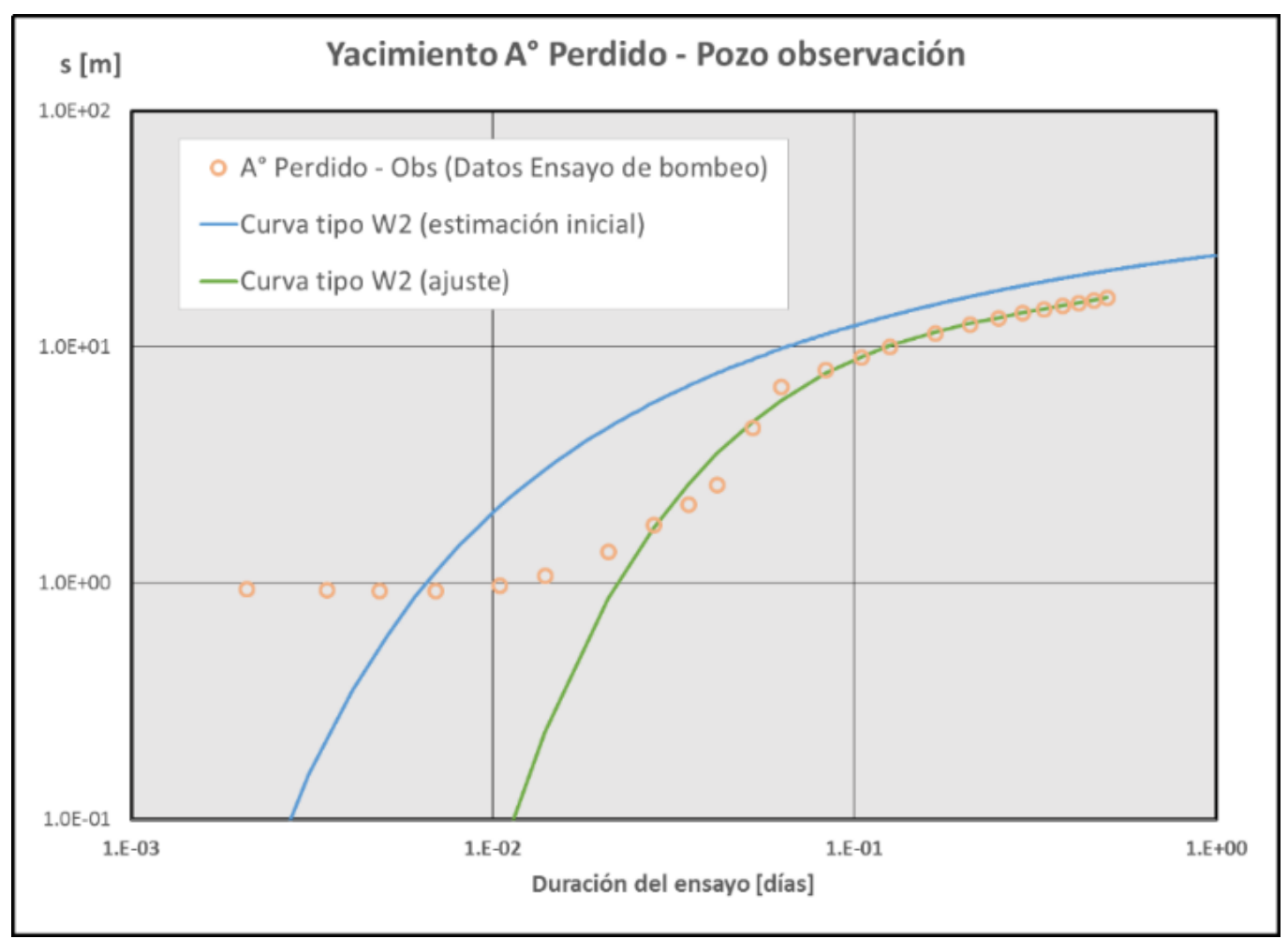

Figura 4.12: Datos del Ensayo de bombeo y ajuste de la W2DP en el sitio Arroyo Perdido. 
Finalmente, los parámetros de la Función de Transferencia que surgen de los ajustes realizados son los siguientes:

Tabla 4.2: Parámetros de la Función de transferencia matriz -fisura

\begin{tabular}{lccc}
\hline Sitio ensayado & $\begin{array}{c}\alpha \\
{[1 / \mathrm{día}]}\end{array}$ & $\begin{array}{c}\alpha \\
{\left[1 / \mathrm{m}^{2}\right]}\end{array}$ & $\begin{array}{c}b \\
{[\mathrm{~m}]}\end{array}$ \\
\hline Cerro Solo & $8,6.10^{-3}$ & $3,0.10^{-4}$ & 97,7 \\
Puesto Alvear & $5,4.10^{-2}$ & $3,0.10^{-4}$ & 98,0 \\
El Molino & $2,8.10^{-3}$ & $3,0.10^{-2}$ & 60,0 \\
Arroyo Perdido & $4,9.10^{-4}$ & $3,0.10^{-2}$ & 75,0 \\
\hline
\end{tabular}

De esta manera, se ha podido comprobar mediante la aplicación de la solución analítica aportada por De Smedt (2011) que la doble porosidad es una propiedad fundamental del acuífero bajo estudio.

En efecto, la identificación de los parámetros de la función de transferencia matriz-fisura (Tabla 4.2) aporta un conocimiento adicional que es susceptible de ser escalado desde sus valores locales (definidos mediante los ensayos de bombeo) a una distribución regional que mejore la modelización del flujo en estos acuíferos complejos. El mismo concepto puede extenderse a los valores de los parámetros mostrados en la Tabla 3.1, diferenciando en forma explícita los rangos de cada componente (matriz-fisura) del sistema acuífero.

Finalmente, se abre así la posibilidad de utilizar la simulación geoestadística de los parámetros que definen dicha función de transferencia de forma tal de lograr un tratamiento adecuado de la fuerte anisotropía y heterogeneidad del medio subterráneo estudiado.

Recurriendo entonces al trabajo antecedentes de Hernández et al. (2012) se pueden identificar en la zona de trabajo tres tipos de fracturas: una falla regional o megafalla de orientación W-E; una fractura denominada Gastre de orientaciones conjugadas N55W - N55E y una fractura denominada Comallo de orientaciones conjugadas N15W y N35E. En el primer caso el buzamiento es casi vertical, y en los otros dos casos, el plano de inclinación es de $40^{\circ}$ con orientación al SW. La simulación con la herramienta SISIM de las distintas combinaciones de fracturas presentes en la región (para una proporción del 5\% cada una), dan los siguientes resultados del Factor de Forma:

Tabla 4.3: Factor de forma estimado en el Distrito Cerro Solo

\begin{tabular}{lc}
\hline \multicolumn{1}{c}{ Fractura } & $\begin{array}{c}\alpha \\
{\left[1 / \mathrm{m}^{2}\right]}\end{array}$ \\
\hline [1] Megafalla & $1,0 \times 10^{-2}$ \\
[2] Gastre (d) & $7,1 \times 10^{-2}$ \\
[3] Gastre (i) & $6,8 \times 10^{-2}$ \\
[4] Comallo (d) & $5,8 \times 10^{-2}$ \\
[5] Comallo (i) & $6,2 \times 10^{-2}$ \\
[1] + [2] & $1,1 \times 10^{-1}$ \\
[1] + [4] & $9,5 \times 10^{-2}$ \\
[1] + [3] & $1,1 \times 10^{-1}$ \\
[1] + [5] & $1,0 \times 10^{-1}$ \\
[1] + [2] + [4] & $2,5 \times 10^{-1}$ \\
[1] + [3]+[5] & $2,4 \times 10^{-1}$ \\
\hline
\end{tabular}


En el mapa de la Figura 4.13 se reproducen las estructuras geológicas mencionadas en el trabajo de Hernández et al. (2012) y de Bianchi (1995). Los sectores donde se realizaron los ensayos de bombeo se han marcado con un sombreado en diagonal.
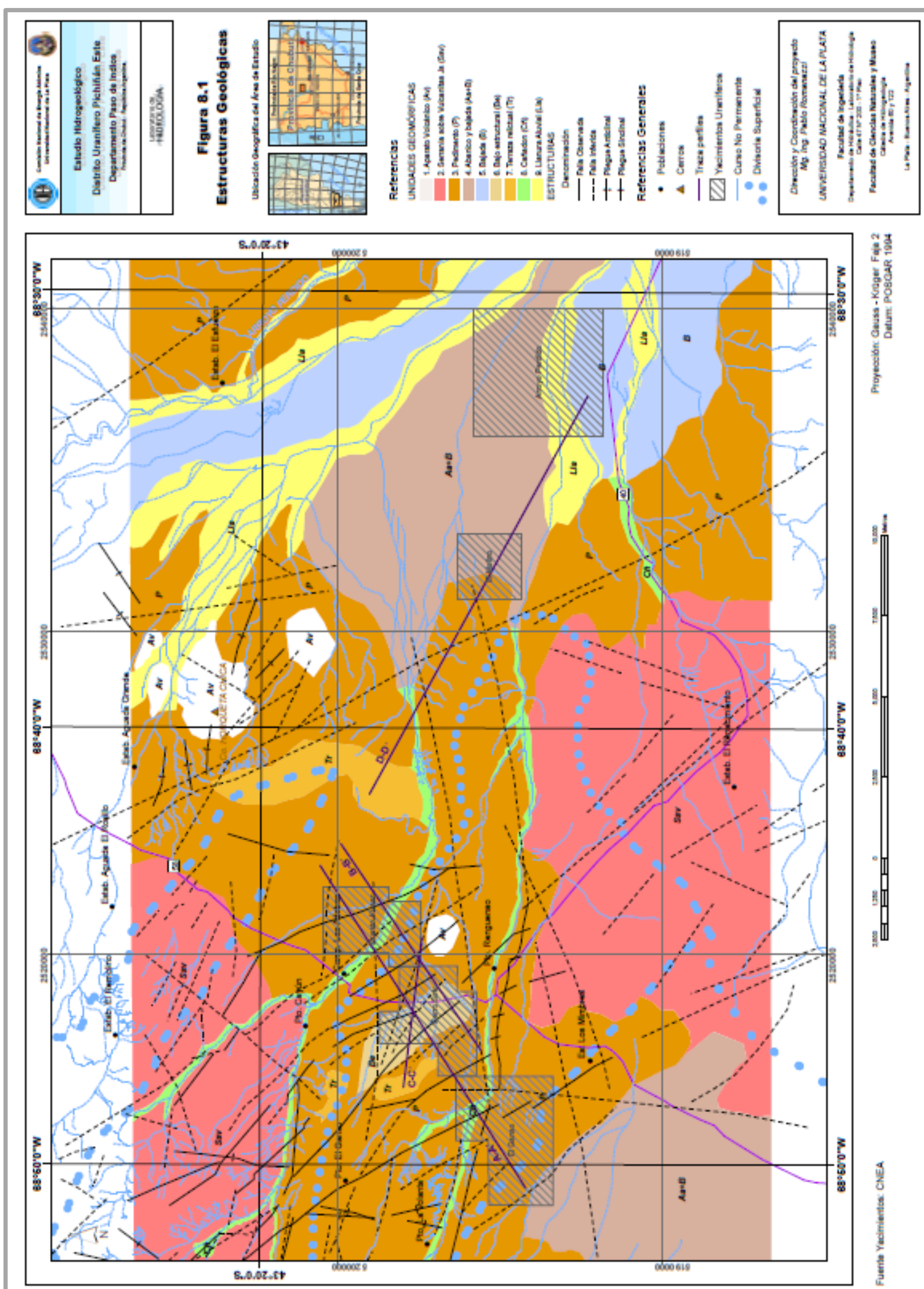

Figura 4.13: Mapa de estructuras geológicas en el Distrito Cerro Solo, Chubut, Argentina. 
Como se aprecia en la tabla 4.3, si la proporción de las fracturas es correcta (en realidad es un porcentaje mínimo para la zona) los valores de la Tabla 4.2 estarían subestimados para los dos sitios ubicados aguas arriba (Cerro Solo y Puesto Alvear) que es justamente donde se verifica la mayor presencia de familias de fracturas. Adoptando $\alpha$ de acuerdo con este nuevo método, surge la posibilidad de estimar los valores de la conductividad de la interfase matriz fisura $\left(\mathrm{K}_{\mathrm{i}}\right)$ tal como se consigna en la siguiente tabla:

Tabla 4.4: Nuevos parámetros de la Función de transferencia matriz -fisura

\begin{tabular}{lcccc}
\hline Sitio ensayado & $\begin{array}{c}\mathrm{C} \\
{[1 / \mathrm{día}]}\end{array}$ & $\begin{array}{c}\alpha \\
{\left[1 / \mathrm{m}^{2}\right]}\end{array}$ & $\begin{array}{c}\mathrm{K}_{\mathrm{i}} \\
{[\mathrm{m} / \mathrm{día}]}\end{array}$ & $\begin{array}{c}\mathrm{b} \\
{[\mathrm{m}]}\end{array}$ \\
\hline Cerro Solo & $8,6.10^{-3}$ & $2,5 \times 10^{-1}$ & $3,5 \times 10^{-4}$ & 97,7 \\
Puesto Alvear & $5,4.10^{-2}$ & $1,1 \times 10^{-1}$ & $5,0 \times 10^{-3}$ & 98,0 \\
El Molino & $2,8.10^{-3}$ & $7,1 \times 10^{-2}$ & $6,6 \times 10^{-4}$ & 60,0 \\
Arroyo Perdido & $4,9.10^{-4}$ & $1,0 \times 10^{-2}$ & $6,5 \times 10^{-4}$ & 75,0 \\
\hline
\end{tabular}

Estos resultados son muy alentadores dado que confirman el comportamiento del acuífero confinado ensayado en cada uno de los sitios referidos en la Tabla 4.4, resultando el pozo de Puesto Alvear el más productivo de todos ellos. A la luz de estos resultados, tal vez favorecido por una conductividad de interface más alta.

No obstante, el valor de $K_{1}$ resultó ser muy uniforme para toda la región, lo cual significa que se puede confirmar para este caso la dependencia intrínseca de este parámetro con la naturaleza de las fases que conecta y no de otras propiedades del medio. 


\section{DISCUSIÓN DE RESULTADOS}

\section{Acerca de la estimación de los coeficientes de la FTMF}

Se ha tratado en esta tesis de mantener una línea objetiva de análisis para la estimación de los coeficientes que integran la FTMF expresada por la ecuación (8). Pero sin duda que en un tema tan complejo como el estudiado la adopción de ciertos criterios para reducir la incertidumbre que rodean a estos parámetros es más que necesaria a la hora de avanzar con una aplicación práctica.

Por esta misma razón el esfuerzo se ha centrado en poder computar en forma sencilla el factor de forma $\alpha$ dado que el mismo es una característica exclusivamente geométrica y propia de la estructura porosa dual. Desde los casos sintéticos, donde la estimación de este factor se puede realizar rápidamente con unas pocas cuentas, hasta la combinación de simulaciones con el software SISIM para el caso de estudio, todo lo realizado es muy sencillo y de suma utilidad práctica.

Queda por supuesto siempre en consideración la incertidumbre que rodea a la orientación y sobre todo a la proporción medida en volumen que ocupa el sistema de fisuras. Con respecto a esto último, cabe destacar la baja proporción de los valores del factor de forma que surgen de las últimas recopilaciones bibliográficas y que fueran reportadas en la tabla 3.2 de este trabajo de tesis. Trabajando con las ecuaciones (52) a (54) se invirtió el cálculo para deducir a partir de ellas las proporciones correspondientes:

Tabla 5.1: Rango de proporciones de fisuras a partir de los valores de $\alpha$ reportados

\begin{tabular}{ccc}
\hline Dimensión & Límite mínimo & Límite máximo \\
\hline 1D & $0.71 \%$ & $1.42 \%$ \\
2D & $0.73 \%$ & $1.65 \%$ \\
3D & $0.93 \%$ & $2.36 \%$ \\
\hline
\end{tabular}

Es muy interesante este resultado porque es una amplitud de proporciones de fisuras muy bajas que no fue explorada con las simulaciones con SISIM. Más aún este programa reportó problemas de funcionamiento cuando se lo quiso utilizar para proporciones de fisuras que partían del $1 \%$ (no así ELLIPSIM pero no representaba fisuras en el bloque matriz). Posiblemente esto sea debido al tamaño del cubo elemental $(1 \times 1 \times 1)$ que para un bloque de $(100 \times 100 \times 50)$ elementos representa un volumen muy grande de fisura. No obstante, siempre prevaleció la idea de que la componente fisura no es solo un plano de discontinuidad sino toda una transición que afecta el flujo de intercambio con la matriz. Esto puede ser salvado con el procedimiento que se reproduce aquí, es decir, utilizando las funciones empíricas (52) a (54) en ese rango indicado.

\section{Acerca del estudio de la formación acuífera en Cerro Solo}

La diferencia entre los valores hallados para un mismo parámetro en distintos sitios de la comarca puede razonarse a partir de las singulares características del medio físico. 
El acuífero contenido en el Miembro El Pajarito de la Formación Los Adobes muestra una considerable anisotropía y heterogeneidad, típica de ambientes fluviales de alta energía. Ello ocurre debido a la presencia de doble porosidad, coexistiendo la primaria (intergranular) con la secundaria de fractura (diaclasas) y por solución (Scasso y Limarino, 1997).

La notable discrepancia de los valores obtenidos a partir de los ensayos, ratifica a su vez el grado de anisotropía y heterogeneidad mencionado, ya que en tramos muy reducidos se pasa de porosidades secundarias mayores (propia de la génesis de disolución química) a valores intermedios en las diaclasas y menores aún en los espacios porales clásticos $\left(K_{f}=4,8 \times 10^{0} \mathrm{~m} / \mathrm{d}\right.$ a $\mathrm{K}_{\mathrm{f}}=1,2 \times 10^{-3} \mathrm{~m} / \mathrm{d}$, Tabla 4.1).

La hidrogeología en regiones con distintos tipos de medio físico (poroso y fisurado) es verdaderamente de muy dificultosa resolución, en especial cuando las anisotropías ocurren a muy corta distancia. Es de destacar que las variaciones reconocidas en la zona son las más importantes de todas las observadas en el ámbito de las Sierras de Pichiñán y un verdadero caso ejemplo de acuífero multimedio.

No obstante lo apuntado, se considera útiles e inéditos los resultados volcados en la Tabla 4.4 porque informan sobre una estimación de una propiedad hidráulica de la interfase matriz-fractura lo cual pueden orientar investigaciones a futuro en una región minera como la estudiada. 


\section{CONCLUSIONES Y RECOMENDACIONES}

\section{Conclusiones}

En este trabajo de tesis la contribución que se hace al conocimiento de los medios geológicos naturalmente fracturados, en particular a aquellos que se identifican como unidades acuíferas, se concentran en una recapitulación de las aproximaciones que se han realizado desde hace ya casi 60 años cuando se enunciaron las primeras teorías de los medios con doble porosidad (Barenblatt et al. 1960) hasta los trabajos de más reciente expresión de las soluciones analíticas para las funciones de pozo (De Smedt, 2011).

Así, la expresión (33), que puede usarse para reemplazar el desarrollo en serie de la función de Theis, es un aporte que luego fue utilizado para desarrollar la expresión del descenso en el caso sintético de una fractura plana vertical (modelo de Gringarten, 1974).

El objetivo principal en toda la tarea de construcción de esta tesis fue encontrar un camino sencillo y muy expeditivo para reducir la incertidumbre que rodean a los coeficientes que conforman la ecuación de transferencia de flujo entre matriz y red de fisuras.

Tanto la metodología propuesta en el punto 3.2.2 como las expresiones (52) a (54) para estimar el factor de forma en función de la proporción de fisuras (punto 3.1.3) van en ese sentido del cumplimiento del objetivo principal.

Sin embargo, al no estar trabajando con datos locales (que no abundan en el campo) de cierta representación del medio que se está estudiando, las simulaciones se pudieron realizar por esa razón en forma medianamente sencilla.

Esto ya al menos significa un aporte como para empezar a acercarse a la compleja simetría que puede presentarse en una red de fracturas. En el caso que existan datos de campo, la vía queda expedita para aplicar de todas formas la metodología propuesta en esta tesis; costará un poco más la pre-elaboración de los datos para cargarlos como datos duros en los programas utilizados para la simulación estocástica de la red de fracturas, pero la vinculación con el coeficiente $C$ de la FTMF es sencilla y directa, razón por la cual se sostiene que lo que aporta este trabajo será de utilidad para futuras investigaciones. 


\section{Recomendaciones}

La aplicación a las ecuaciones de pozo con el diferimiento que se produce en el descenso temprano por agotamiento de las reservas en la red de fisuras y el ritmo que se retoma cuando la matriz cede agua para compensar este déficit, también está relacionado íntimamente con la FTMF. Es por esa razón que se considera útil seguir investigando el contraste de distintas configuraciones de la red de fracturas con los relevamientos de campo a fin de afianzar la regionalización de los parámetros de la FTMF.

La utilidad de la aproximación lograda para evaluar la FTMF en distintos casos de estudio debería seguir siendo contrastada en especial para que no se termine usando como coeficiente global de calibración de un modelo de flujo y que pierda así su significado físico.

Por último, sería muy interesante poder desarrollar a futuro una línea de trabajo que siga este mismo espíritu, pero en los modelos de transporte. Queda así una vía dirigida a amalgamar (como se hizo aquí) la geometría de los contornos de una red simulada por medio de la Geoestadística y una solución global para el transporte de solutos. 


\section{REFERENCIAS}

\subsection{CitadAs en el teXto}

Adler, P.M., Thovert, J-F. y Mourzenko, V.V., (2013); "Fractured porous media"; Oxford University Press.

Ahmed, S., Jayakumar, R. y Salih, A. (Editores), (2008); "Grounwater dynamics in hard rock aquifers", Springer.

Alabert, F. G. (1987); "Stochastic imaging of spatial distributions using hard and soft data"; Master's thesis, Stanford University, Branner Earth Sciences Library.

Arora, B., Monhanty, B.P. y McGuire, J.T., (2012); "Uncertainty in dual permeability model parameters for structured soils"; Water Resources Research, 48(1), W01508, 17pp.

Bai, M. y Elsworth, D. (2000); "Coupled processes in subsurface Deformation, Flow and Transport", ed. ASCE Press.

Bakalowicz, $M$ (2005); "Karst groundwater: a challenge for new resources"; Hydrogeology Journal, 13:148-160.

Barker, J., (1985); "Generalized well function evaluation for homogeneous and fissured aquifers"; Journal of Hydrology, (76):143-154.

Batu, V. (1998); "Aquifer Hydraulics: a comprehensive guide to hydrogeologic data analysis", ed. J. Wiley.

Barenblatt, G.I., Zheltov, Iu.P. y Kochina, I.N., (1960); "Basic concepts in the theory of seepage of homogeneous liquids in fissured rocks"; Journal of Applied Mathematics and Mechanics, 24(5):1286-1303.

Bear, J. (1972); "Dynamics of fluids in porous media"; Ed. Dover Publications.

Bianchi, R. E. (1995). Informe estructural del Yacimiento Cerro Solo. Comisión Nacional de Energía Atómica. Gerencia de exploración. Departamento Regional Patagonia. (Inédito)

Bianchi, R. y Benítez, A. (1994); "Relevamiento hidrogeológico, Yacimiento Cerro Solo y Vertiente Oriental del Distrito Pichiñán Este", informe técnico (inédito), CNEA.

Bogdanov I.I., Mourzenko, V.V., Thovert, J.F. y Adler, P.M., (2003); "Two-phase flow through fractured porous media"; Physical Review, E68 (026703):1-24.

Bogdanov I.I., Mourzenko, V.V., Thovert, J.F. y Adler, P.M., (2007); "Effective permeability of fractured porous media with power-law distribution of fractures sizes"; Physical Review, E76 (036309):1-15.

Bogdanov I.I., Mourzenko, V.V., Thovert, J.F. y Adler, P.M., (2003); "Effective permeability of fractured porous media in steady state flow"; Water Resources Research, 39 (1):1-16. 
Bogdanov I.I., Mourzenko, V.V., Thovert, J.F. y Adler, P.M., (2003); "Pressure drawdown well test in fractured porous media"; Water Resources Research, Vol. 39 (1):SBH11-1 a 19.

Bogatkov, D, y Babadagli, T (2010); " Fracture network modeling conditioned to pressure transient and tracer test dynamic data"; Journal of Petroleum Science and Engineering, 75:154-167.

Boulton, N.S. y Streltsova, T.D., (1977a); "Unsteady Flow to a Pumped Well in a twolayered water bearing formation"; Journal of Hydrology, 35:245-256.

Boulton, N.S. y Streltsova, T.D., (1977b); "Unsteady Flow to a Pumped Well in a Fissured water bearing formation"; Journal of Hydrology, 35:257-270.

Boulton, N. y Streltsova, T., (1978a); "Unsteady flow to a pumped well in an unconfined fissured aquifer"; Journal of Hydrology, 37:349-363.

Boulton, N.S. y Streltsova, T.D., (1978b); "Unsteady Flow to a Pumped Well in a Fissured Aquifer With a Free Surface Level Maintained Constant"; Water Resources Research, 14(3):527-532.

Bourbiaux, B. et al. (1998); "A rapid and efficient methodology to convert fractured reservoir images into a dual porosity model"; Revue de l'Institut français du pétrole, vol. 53, $N^{\circ} 6, p p .785-799$.

Cassiraga, E. y Gómez-Hernández, J. J. (1996) "Métodos geoestadísticos para la integración de la información"; Publicación técnica 04/96 ENRESA.

Cassiraga, E. F. y Gómez-Hernández, J. J. (2001); “Utilización de técnicas geoestadísticas para la generación de medios fracturados en 3D"; Hidrogeología y Aguas Subterráneas, Ministerio de Ciencia y Tecnología - Instituto Geológico y Minero de España, (I)341-345.

Chang, Y. y Yeh, H., (2011); "Skin effect in generalized radial flow model in fractured media. Geophysical Journal International", 185:78-84.

Choi, E.S., Cheema, T. e Islam, M.R. (1997); "A new dual-porosity/dual-permeability model with non-Darcian flow through fractures"; Journal of Petroleum Science and Engineering, 17:331-344.

Cinco, H. et al., (1978); "Transient pressure behavior for a well with a finite-conductivity vertical fracture", SPE, 253-264.

Civan, F. (2011); "Porous media transport phenomena"; Ed. Wiley.

Cushman, J. H. (editor, 1990); "Dynamics of fluids in hierarchical porous media"; Ed. Academic Press. En especial, el capítulo VII denominado "Dual porosity models for flow in naturally fractured reservoirs" de J. Douglas y T. Arbogast, pág. 177-222.

Custodio, E. y Llamas, M. R. (1996); "Hidrología Subterránea”; Ed. Omega.

Dagan, G. y Neuman, S. P. (1997); "Subsurface flow and Transport: a stochastic approach"; International Hydrology Series, IASH/AISH, Cambridge University Press. En particular el artículo "Component characterization: an approach to fracture hydrogeology", de J. C. S. Long, C. Doughty, A. Datta-Gupta, K. Hestir y D. Vasco, pág. 179-195. 
Darcy, H., (1856); "Determination of the laws of water flow through sand", The public fountains of the City of Dijon, Appendix D - Filtration; en Benchmark papers in Hrdology, capítulo "Groundwatre", IAHS Press, 2008.

Delgado, J. (Editor), (2012); "Heat \&mass transfer in porous media"; Advanced structured materials, Vol. 13, Springer.

Delleur, J. (editor, 1999); "The Handbook of Groundwater Engineering", Ed. CRC Press. Con referencia directa al Capítulo 17, denominado "Groundwater flow and solute transport in fractured media" de P.A. Lapcevic, K. S. Novakowski and E. A. Sudicky, 17/1-39.

De Marsily, Gh. (1986); "Quantitative Hydrogeology: groundwater hydrology for engineers", ed. Academic Press.

De Marsily, Gh., Delay,F et al. (2005); "Dealing with spatial heterogeneity", Hydrogeological Journal, 13:161-183.

De Smedt, F. y Wierenga, P.J., (1979); "A generalized solution for solute flow in soils with mobile and immobile water"; WRR 15(5), 1137-1141.

De Smedt, F. (2011). Analytical solution for constant-rate pumping test in fissured porous media with double-porosity. Transport in Porous Media, 88:479-489.

De Swaan, O. (1976); "Analytic solutions for determining naturally fractured reservoir properties by well testing", SPE, 117-1122

Deutsch, C.V. y Journel, A. G. (1998); "GSLIB - Geostatistical software library and user's guide"; 2da. Edición, Oxford University Press.

Dietrich, P. , Helmig, R., Sauter, M., Hötzl, H., Köngeter, J. y Teutsch, G. (Editores), (2005); "Flow and transport in fractured porous media"; Springer.

Douglas, J. y Arbogast, T., (1990); "Dual porosity models for flow in naturally fractured reservoirs", en Dynamics of fluids in hierarchical porous media, editado por J.H. Cushman, Academic Press.

Duguid, J.O. y Lee, P.C.Y. (1977); "Flow in fractured porous media"; Water Resources Research, Vol. 13(3):558-566.

Freeze, R.A. (1975); "A stochastic-conceptual analysis of one-dimensional groundwater flow in nonuniform homogeneous media"; Water Resources Research, Vol. 11(5):725-741.

Gerke, H.H. (1996); "Macroscopic representation of structural geometry for simulating water and solute movement in dual porosity media"; Advances in Water Resources, Vol. 19, No. 6, pp. 343-351.

Gerke, H.H. y Van Genuchten, M.T., (1993a); "Evaluation of a first-order water transfer term for variably saturated dual-porosity flow models. Water Resources Research, 29(4):1225-1238.

Gerke, H.H. y Van Genuchten, M.T., (1993b); "A dual-porosity model for simulating the preferential movement of water and solutes in structured porous media. Water Resources Research, 29(2):305-319. 
Goldscheider, N. y Drew, D. (editors, 2007); "Methods in Karst Hydrogeology", Ed. Taylor \& Francis Group.

Gómez-Hernández, J. J. (1991) "Geoestadística para el análisis de riesgos. Una introducción a la geoestadística no paramétrica"; Publicación técnica 04/91 ENRESA.

Gómez-Hernández y Cassiraga, E. F. (1994); "Theory and practice of sequential simulation", en Geostatistical Simulations, M. Armstrong and P. A. Dowd (eds.), Kluwer Academic Press, 111-124.

Gómez-Hernández, J. J. y Journel, A. J. (1993); "Joint sequential simulation of multiGaussian fields", en Geostatistics Troia '92, A. Soares (ed.), Kluwer Academic Publ., vol. 1, 85-94.

Gómez-Hernández, J.J., Sahuquillo, A. y Capilla, J. (1997); "Stochastic simulation of transmissivity fields conditional to both transmissivity and piezometric data - I. Theory"; Journal of Hydrology, 203:162- 174.

Gómez-Hernández, J. J. y Srivastava, R. M. (1990); "ISIM3D: An ANSI-C three-dimensional multiple indicator conditional simulation program"; Computer and Geosciences, 16(4), 395-440.

Greene, E. A., (1993); "Hydraulic properties of the Madison Aquifer System in the western Rapid City area, South Dakota, USGS, Water resources investigations report 93-4008.

Gringarten, A. C., E. A., (1974); "Unsteady-state pressure distributions created by a well with a single infinite-conductivity vertical fracture", SPE, 347-360.

Hantush, M. S. (1964); "Hydraulics of wells"; Advances in Hydrosciencies, 1:281-432.

Hantush, M. S. y Thomas, R.G. (1966); "A method for analyzing a drawdown test in anisotropic aquifers"; Water Resources Research, Vol. 2-2:281-285

Hassanzadeh, H. , Pooladi-Darvish, M. y Atabay, S. (2009); "Shape factor in the drawdown solution for well testing of dual-porosity systems"; Advances in Water Resources, 32:16521663.

Hassanzadeh, H. y Pooladi-Darvish, M., (2006); "Effects of fracture boundary conditions on matrix-fracture transfer shape factor. Transport in Porous Media, 64:51-71.

Hendricks-Franssen, H. J., Cassiraga, E. F. Gómez Hernández, J.J., Sahuquillo, A. y Capilla, J. E. (1998); "Inverse modeling of groundwater flow in a fractured media"; Proceedings of GeoEnv II, Ed. Kluger Academic Publishers, pp. 283-294.

Hernández, M., Romanazzi, P., González, N. y Trovatto, M. M., (2012). Estudio Hidrogeológico del Distrito Uranífero Pichiñán Este, Paso de Indios, Provincia de Chubut. Informe Final. Laboratorio de Hidrología, Facultad de Ingeniería, Universidad Nacional de La Plata (UNLP), La Plata, Argentina.

Hunt, A. y Ewing, R. (2009); "Percolation theory for flow in porous media"; Lecture notes in physics, Vol. 771, Springer.

Huyakorn, P.S., Lester, B.H. y Faust, Ch. R. (1983); "Finite element techniques for modeling groundwater flow in fractured aquifers"; Water Resources Reserch, Vol. 19 (4), pág. 10191034. 
Hyndman, D. W. y Day-Lewis, F. D. (editors, 2007); "Subsurface Hydrology: data integration for properties and processes", Geophysical Monograph 171, ed. American Geophysical Union.

Isaaks, E. H. (1990); "The application of Monte-Carlo methods to the analysis of spatially correlated data"; PhD thesis, Stanford University, Branner Earth Sciences Library.

Journel, A. G. (1989); "Fundamentals of geostatistics in five lessons"; Short course in geology, vol. 8, American Geophysical Union.

Journel, A. y Alabert, F. G. (1989); "Non-Gaussian data expansion in the earth sciences"; Terra Nova, 1:123-34.

Kazemi, H. (1969); "Pressure transient analysis of naturally fractured reservoirs with uniform fracture distribution"; SPE 2156A, 451-462.

Klimczak, C. et al. (2010); "Cubic law with aperture-length correlation: implications for network scale fluid flow"; Hydrogeology Journal, 18: 851-862.

Koike, K., Liu, C. y Sanga,T. (2012); "Incorporation of fracture directions into 3D geostatistical methods for a rock fracture system"; Environ Earth Sci, 66:1403-1414.

Krásny, J. (2003); "Distribución espacial de los parámetros hidráulicos en diversos medios hidrogeológicos"; Boletín Geológico y Minero, 114 (2): 171-182.

Kresic, N. (2007); "Hydrogeology and Ground Water modeling", Ed. CRC Press, 2 a edición. Referencia al artículo 2.3.2 titulado "Fractured rocks and karst aquifers", pág. 118-126.

Lai, K.S. y Pao, W.K.S, (2013); "Assessment of different matrix-fracture shape factor in double porosity medium"; Journal of Applied Sciencies, 13(2):308-314.

Langevin, C. D. (2002); "Stochastic ground water flow simulation with a fracture zone continuum model"; Ground Water, Vol. 41(5):587-601.

Lemonnier, P. y Bourbiaux, B., (2010); "Simulation of Naturally Fractured Reservoirs. State of the Art"; Oil \& Gas Science and Technology - Rev. IFP, 65(2):239-262

Lim, K.T. y Aziz, K., (1995); "Matrix-fracture transfer shape factors for dual-porosity simulators"; Journal of Petroleum Science and Engineering, 13:169-178.

Lu, M. y Connell, L.D. (2011); "A Statistical Representation of the Matrix-Fracture Transfer Function for Porous Media"; Transp. Porous Med., 86:777-803.

Marquardt, D.W., (1963); "An algorithm for least-squares estimation of non-linear parameters", S.I.A.M. 11, 431-441.

Matheron, G. (1965); "Las variables regionalizadas y su estimación"; Tesis presentada a la Facultad de Ciencias de Paris, Francia. Traducción de Marco Alfaro, 2008.

Moench, A.F., (1984); " Double-porosity models for a fissured groundwater reservoir with fracture skin"; Water Resources Research, 20(7):831-846.

Najurieta, H. L. (1980); "A theory for pressure transient analysis in naturally fravtured reservoirs"; Journal of Petroleum Engineering, SPE, 1241-1250. 
Neuman, S. P. (1974); "Effect of partial penetration on flow in unconfined aquifers considering delayed gravity response"; Water Resources Research, 10(2):303-312.

Neuman, S. P. (2005); "Trends, prospects and challenges in quantifying flow and transport through fractured rocks"; Hydrogeol J., 13:124-147

Nitao, J.J. y Buscheck, T.A., (1991); "Infiltration of a liquid front in an unsaturated fractured porous medium"; Water Resources Research, 27(8):2099-2112.

Nonner, J. (2010); "Introduction to Hydrogeology"; UNESCO-IHE lecture note series, ed. CRC Press. En especial su capítulo 4, "Water Balances", pág. 135-168.

Nowak, W., Schwede, R.L., Cirpka, O.A. y Neuweiler, I. (2008); "Probability density functions of hydraulic head and velocity in three-dimensional heterogeneous porous media"; Water Resour. Res., 44, W08452.

Pinder, G. F. y Celia, M. A. (2006); "Subsurface Hydrology"; Ed. J. Wiley.

Presho, M. et al. (2011); "Calibrated dual porosity, dual permeability modeling of fractured reservoirs"; Journal of Petroleum Science and Engineering, 77:326-337.

Ranjbar, E. y Hassanzadeh, H. (2011); "Matrix-fracture transfer shape factor for modeling flow of a compressible fluid in dual-porosity media"; Advances in Water Resources, 34:627-639.

Ranjbar, E., Hassanzadeh, H. y Chen, Z, (2011); "Effect of fracture pressure depletion on a dual porosity shape factor for flow of compressible fluids in fractured porous media"; Adv. in Water Resources, 34:1681-1693.

Remy, N., Boucher, A. y Wu, J., (2009); "Applied Geostatistics with SGeMS - A user's guide"; Cambridge University Press.

Renar, Ph. y de Marsili, G., (1997); "Calculating equivalent permeability: a review"; Advances in Water Resources, 20(5-6):253-278.

Romanazzi, P. y Cassiraga, E. (2004a); "Una propuesta para la incorporación de la función de transferencia matriz-fisura en modelos de doble porosidad"; XII Congreso Latinoamericano de HIdrogeología, Santiago, Chile, agosto de 2014.

Romanazzi, P. y Cassiraga, E. (2004b); "Estado del arte de la simulación de la función de transferencia matriz - fractura en modelos de acuíferos con doble porosidad"; II Congreso Internacional de Hidrología de Llanuras, Santa Fe, Argentina, septiembre de 2014.

Romanazzi, P. y Cassiraga, E. (2004c); "Regionalización de los parámetros hidrogeológicos en un acuífero con doble porosidad"; II Congreso Ibérico de las Aguas Subterráneas, CIAS_2014, Valencia, septiembre de 2014

Royer,P. , Auriault, J.L. y Boutin, C. (1996); "Macroscopic modeling of double-porosity reservoirs"; Journal of Petroleum Science and Engineering, 16:187-202.

Rubin, Y. (2003); "Applied stochastic hydrogeology", ed. Oxford University Press. En especial el capitulo 6 referido a "Upscaling, computational aspects, and statistics of the velocity field", pág. 140-168. 
Sahimi, M. (2011); "Flow and Transport in porous media and fractured rock"; 2a Ed., WileyVCH, Weinheim.

Scasso, R. A. y C. O. Limarino, (1997); "Petrologìa y Diagénesis de Rocas Clásticas". Publ. Especial №1. Asoc. Arg. de Sedimentologìa, 259 pág. Bs. As.

Scesi, L. y Gattinoni, P. (2007); "Roughness control on hydraulic conductivity in fractured rocks"; Hydrogeology Journal, 15: 201-211.

Scesi, L. y Gattinoni, P. (2009); "Water Circulation in Rocks"; Springer.

Sen, Z., (2009); "Spatial modeling principles in earth sciencies"; Springer.

Singhal, B.B.S. y Gupta, R.P., 2010; "Applied hydrogeology of fractured rocks"; 2a. edición, Springer.

Sternberg, Y.M., (1969); "Some approximate solutions of radial flow problems"; Journal of Hydrology, 7:158-166.

Streltsova, T.D., (1976a); "Hydrodynamics of groundwater flow in a fractured formation"; Water Resources Research, 12(3):405-414.

Streltsova, T.D., (1976b); "Analysis of aquifer-aquitard flow"; Water Resources Research, 12(3):415-422.

Streltsova, T.D., (1978); "Well Hydraulics in heterogeneous aquifer formations"; Advances in Hydrosciencies, 11:357-423.

Streltsova, T.D., (1984); "Well Hydraulics in heterogeneous porous media", en Fundamentals of Transport Phenomena in porous media, ed. Por Bear,J. y Corapcioglu, M.Y., NATO ASI Series, 82:317-346.

Schwartz, F. W. y Zhang, H. (2003); "Fundamentals of ground water"; Ed. J. Wiley.

Stadler, L., Hinkelmann, R. y Helmig, R, (2012); "Modeling Macro-porous Soils with a TwoPhase Dual-Permeability Model"; Transp. Porous Media, 95:585-601.

Theis, C. V. (1935); "The relation between the lowering of the piezometric surface and the rate and duration of the discharge of a well using groundwater storage"; EOS Trans AGU, 16:519-524.

Vert, M. et al. (1998); "Geostatistical generation of 3-dimensional aquifer realizations using the conditional SIS approach with direction trends imposed on variogram models"; Proceedings of GeoEnv II, Ed. Kluger Academic Publishers, pp. 283-294.

Warren, J.E. y Root, (1963); "The behavior of naturally fractured reservoirs"; Society of Petroleum Engineers Journal, ASME 228:245-255.

Wilson, C.R. y Witherspoon, P.A. (1974); "Steady state flow In rigid network of fractures"; Water Resources Research, 10(2):328-335.

Wu, Y.S. et al. (2004); "A physically based approach for modeling multiphase fracturematrix interaction in fractured porous media"; Advances in Water Resources, 27:875-887. 
Yuan, S.C. y Harrison, J.P., (2006); "A review of the state of art in modelling progressive mechanical breakdown and associated fluid flow in intact heterogeneous rocks"; Journal of Rock Mechanics and Mining Sciences, 43:1001:1022.

Zimmerman, R.W., Chen, G., Hadgu, T. y Bodvarsson, G.S., (1993); "A numerical model with semi-analytical treatment of fracture/matrix flow"; Water Resources Research, 29(7):2127-2137.

Zimmerman, R.W. y Bodvarsson, G.S., (1994); "Hydraulic Conductivity of Rock Fractures"; Lawrence Berkeley Laboratory, University of California, Earth Sciences Division, LBL-35976.

Zimmerman, R.W. y y Bodvarsson, G.S., (1996); "Effective transmissivity of twodimensional fracture networks"; Int. J. Rock Mech. Min. Sci. \& Geomech, 33(4)4:433-438.

Zhang, D. (2002); "Stochastic methods for flow porous media: coping with uncertainties"; Academic Press.

Zhang, D. y Sun,A. Y., (2000); "Stochastic analysis of transient saturated flow through heterogeneous fractures porous media - A double permeability approach"; Water Resources Research, 36(4):865-874.

\subsection{REFERENCIAS CONSULTADAS}

Alaoui, A. y Eugster, W. (2004); "Dual-porosity modeling of groundwater recharge: testing a quick calibration using in situ moisture measurements, Areuse River Delta, Switzerland"; Hydrogeology Journal, 12:464-475.

Alboin, C. et al. (2002); "A comparison of methods for calculating the matrix block source term in a double porosity model for contaminant transport"; Computational Geosciences, 6: 523-543.

Blessent, D. et al. (2011); "Large-scale numerical simulation of groundwater flow and solute transport in discretely-fractured crystalline bedrock"; Adv. in Water Resources, 34: 1539-1552.

Budd, D. A. y Vacher, H. L. (2004); "Matrix permeability of the confined Floridan Aquifer, Florida, USA"; Hydrogeology Journal, 12:531-549.

Carrera, J. et al. (2005); "Inverse problem in hydrogeology"; Hydrogeology Journal, 13:206-222

Chen, Z. (2007); "Homogenization and simulation for compositional flow in naturally fractured reservoirs"; J. Math. Anal. Appl. 326, 12-32.

Cherpeau, N., Caumon, G., Caers, J. y Lévy, B. (2012); "Method for Stochastic Inverse Modeling of Fault Geometry and Connectivity Using Flow Data"; Math Geosci, 44:147-168.

Coffield, D.J. y Spagnuolo, A.M. (2010); "A model for single-phase flow in layered porous media"; Electronic Journal of Differential Equations, Vol. 2010, No. 94, pp. 1-19.

Cuador-Gill, J. Q. y Quintero-Silverio, A. (2001); "Simulación condicional de variables regionalizadas y su aplicación al comportamiento de la porosidad efectiva en un yacimiento fracturado-poroso"; Boletín de la Sociedad Geológica Mexicana, Tomo LIV, pp. 19-27. 
dell'Arciprete, D. et al. (2012); "Comparison of three geostatistical methods for hydrofacies simulation: a test on alluvial sediments"; Hydrogeology Journal, 20: 299-311.

Dewandel, B. et al. (2005); "A conceptual hydrogeological model of ophiolite hard-rock aquifers in Oman based on a multiscale and a multidisciplinary approach"; Hydrogeology Journal; 13: 708-726.

Dougherty, D. y Babu, D., (1984); "Flow to a partially penetrating well in a double-porosity reservoir"; Water Resources Research, 20(8):1116-1122.

Douglas, J. et al. (1997); "Single Phase Flow in Partially Fissured Media"; Transport in Porous Media, 28: 285-306.

Ehsan Samimi, S., Mohsen Masihi, M., Shahab Gerami, S. y Ganjeh Ghazvini, M. (2012); "An Improvement on modeling of Forced Gravity Drainage in Dual Porosity Simulations Using a New Matrix-Fracture Transfer Function"; Transp. Porous Med., 94:207-223.

Fowler, A. (2011); " Mathematical Geoscience"; Springer-Verlag London.

Frind, E.O. y Verge, M.J. (1978); "Three- dimensional modeling of groundwater flow systems"; Water Resources Research, Vol. 14(5):844-856.

Gómez-Hernández, J. J. (1991); "A stochastic approach to the simulation of block conductivity fields conditioned upon data measured at smaller scale"; PhD Thesis, Standford University, CA, $351 \mathrm{pp}$.

Hantush, M. S. (1966); "Wells in homogeneous anisotropic aquifers"; Water Resources Research, Vol. 2-2:273-279.

Hendricks-Franssen, H. J. (2000); "Inverse stochastic modeling of groundwater flow and mass transport", PhD Thesis, DIHMA, Universidad Politécnica de Valencia.

Hinkelmann, F. (2005); "Efficient numerical methods and information processing techniques for modeling hydro- and environmental systems"; Lecture notes in applied and computational mechanics, Vol. 21, Springer.

Horne, R. N. (1997); "Modern well test analysis", Petroway Inc., 2a edición.

Huang, W. et al. (2004); "Comparison of streamline-based and grid-based dual porosity simulation"; Journal of Petroleum Science and Engineering, 43: 129- 137.

Huyakorn, P.S. (1986); "A three-dimensional finite-element model for simulating water flow in variably saturated porous media"; Water Resources Reserch, Vol. 22 (13), pág. 17901-1808.

Huyakorn, P.S., Jones, B.G. y Andersen, P. F. (1986); "Finite element algorithms for simulating three-dimensional groundwater flow and solute transport in multilayer systems"; Water Resources Reserch, Vol. 22 (3):361-374.

Huysmans, M. y Dassargues, A. (2009); "Application of multiple-point geostatistics on modelling groundwater flow and transport in a cross-bedded aquifer (Belgium)"; Hydrogeology Journal, 17: 1901-1911. 
Koyama, T. , Li, B., Jiang, Y. y Jing, L.; (2009); "Numerical modelling of fluid flow tests in a rock fracture with a special algorithm for contact areas"; Computers and Geotechnics, 36:291-303.

Le Ravalec-Dupin, Mickaêle (2005); "Inverse stochastic modeling of flow in porous media: Application to reservoir characterization"; Ed. Technip.

Llerar-Meza, G. (2009); "Upscaling non-reactive solute transport"; PhD Tesis, Grupo de Hidrogeología, Universidad Politécnica de Valencia (UPV).

Llopis-Albert, C. y Capilla, J. E. (2010); "Stochastic inverse modelling of hydraulic conductivity fields taking into account independent stochastic structures: a 3D case study"; J. of Hydrology 391, 277-288.

Lu, M. y Connell, L.D. (2006); "A Note on the Characteristic Length/Time of Dual-Porosity Models for Geologically Fractured Media"; Key Engineering Materials, Vol. 312:263-268.

Mejía Rivera, O. et al. (2007); "Aplicación de técnicas geoestadísticas en la hidrogeología del Bajo Cauca Antioqueño"; Dyna, UNC, Año 74, Nro. 152, pp137-149.

Moutsopoulos, K. et al. (2001); "Hydraulic Behavior and Contaminant Transport in Multiple Porosity Media"; Transport in Porous Media, 42: 265-292.

Narasimhan, T.N. y Witherspoon, P.A. (1976); "An Integrated Finite Difference Method for Analyzing Fluid Flow in porous media"; WRR, 12(1):57-64.

Neuman, S.P. y Witherspoon, P.A., (1969a); "Theory of flow in a confined two aquifer system"; Water Resources Research, Vol. 5 (4):803-816.

Neuman, S.P. y Witherspoon, P.A., (1969b); "Applicability of current theories of flow in leaky aquifers"; Water Resources Research, Vol. 5 (4):817-829.

Nielsen, Kurt A. (2007); "Fractured aquifers: formation evaluation by well testing"; Ed.Trafford.

Painter S. L. et al. (2007); "Transmissivity estimation for highly heterogeneous aquifers: comparison of three methods applied to the Edwards Aquifer, Texas, USA"; Hydrogeology Journal, 15: 315-331.

Park, E. y Zhanb, H. (2003); "Hydraulics of horizontal wells in fractured shallow aquifer systems"; Journal of Hydrology, 281:147-158.

Pinder, G. F. y Gray, W. G. (1977); "Finite element simulation in surface and subsurface hydrology"; Academic Press.

Reichard, J, S, y Leap, D. I. (1998); "The effect of pore pressure on the conductivity of fractured aquifers"; Ground Water, Vol. 36, Nro. 3, pp. 450-456.

Rosensheim, J. y Bennett, G.D. (editores, 1986); "Groundwater Hydraulics"; American Geophysical Union, Water Resources Monograph 9, 2 a edición. En especial, el artículo denominado "Pumping test analysis in fractured aquifer formations" de C. Sauveplane, pág. 171-206.

Sabet, M. A. (1991); "Well Test Analysis"; Contributions in Petroleum Geology and Engineering, Vol. 8, GPC. 
Samper Calvete, F. J. y Carrera Ramírez, J. (1990); "Geoestadítica: Aplicaciones a la Hidrología Subterránea"; Centro Internacional de Métodos Numéricos, Universidad de Catalunya, Barcelona.

Sahimi, M. et al. (2010); "Upscaled Unstructured Computational Grids for Efficient Simulation of Flow in Fractured Porous Media"; Transp Porous Med, 83:195-218.

Sedghi, M. y Samani, N., (2015); "Semi-analytical solutions for flow to a well in an unconfined-fractured aquifer system"; Advances in water resources, (83):89-101.

Shi, P., Spagnuolo, A. y Wright, S. (2005); "Reiterated Homogenization and the DoublePorosity Model; Transport in Porous Media, 59:73-95.

Stephan, E. y Wriggers, P. (Editores), (2011); "Modelling, simulation and software concepts for scientific-technological problems"; Lecture notes in applied and computational mechanics, Vol. 57, Springer.

Streltsova, T.D., (1972); "Unsteady radial flow in an unconfined aquifer"; Water Resources Research, 8(4):1059-1066.

Streltsova, T.D., (1973); "Flow near a pumped well in an unconfined aquifer under nonsteady conditions"; Water Resources Research, 9(1):227-235.

Streltsova, T.D. y Rushton, K. R., (1973); "Water table drawdown due to a pumped well in an unconfined aquifer"; Water Resources Research, 9(1):236-242.

Sudicky, E.A. y McLaren, R.G. (1992); "The Laplace transform Galerkin technique for large scale simulation of mass transport in discretely fractured porous formations"; Water Resources Reserch, Vol. 28 (2), pág. 499-514.

Therrien, R. y Sudicky, E.A. (1996); "Three-dimensional analysis of variably-saturated flow and solute transport in discretely-fractured porous media"; Journal of Contaminant Hydrology, 23:1-44

Tsakirogloua, C.D. et al (2009); "A new approach for the characterization of the pore structure of dual porosity rocks"; Chemical Engineering Science, 64:847-859.

Troisi, S. et al. (2000); "Application of kriging with external drift to estimate hydraulic conductivity from electrical-resistivity data in unconsolidated deposits near Montalto Uffugo, Italy"; Hydrogeology Journal, 8:356-367.

Vogel, T., Gerke, H.H., Zhang, R. y Van Genuchten, M. Th. (2000); "Modeling flow and transport in a two-dimensional dual-permeability system with spatially variable hydraulic properties"; Journal of Hydrology, 238:78-89.

Wang, E.Z., Yue, Z.Q., Tham, L.G., Tsui,Y. y Wang,H.T. (2002); " A dual fracture model to simulate large-scale flow through fractured rocks"; Can. Geotech. J. 39: 1302-1312.

Wang, J.S.Y. y Narasimhan, T.N. (1985); "Hydrologic mechanism governing fluid flow in a partially saturated, fractured, porous medium"; Water Resources Research, Vol. 21 (12):1861-1874.

Wang, M., Kulatilake, P.H.S.W., Um, J. y Narvaiz, J. (2002); "Estimation of REV size and three-dimensional hydraulic conductivity tensor for a fractured rock mass through a single 
well packer test and discrete fracture fluid flow modeling"; International Journal of Rock Mechanics \& Mining Sciences, 39:887-904.

Wheater, H. S., Mathias, S.A. y Li, X. (2010); "Groundwater modeling in arid and semi-arid areas"; International Hydrology series, ed. Cambridge University Press. En especial, el capítulo 4 titulado "Groundwater flow and transport" de J. Carrera y S.A. Mathias, pág. 39-62.

Williams, R.E., (1985); Comment on "Double-Porosity Models for a Fissured Groundwater Reservoir with Fracture Skin" by A. F. Moench. Water Resources Research, 21(6):889-891.

Xian-Huan Wen y J. Jaime Gómez-Hernández (1996); "Upscaling hydraulic conductivities in heterogeneous media: An overview"; Journal of Hydrology, 183: ix-xxxii.

Yao, Y., Yu-ShuWu y Zhang, R. (2012); " The Transient Flow Analysis of Fluid in a Fractal Double-Porosity Reservoir"; Transp. Porous Med., 94:175-187.

Y. Zha et al., (2017); "Quasi-steady state conditions in heterogeneous aquifers during pumping tests", Advances in Water Resources, 1-16.

Zyvoloski, G.A., Robinson, B.A. y Viswanathan, H.S. (2008); "Generalized dual porosity: a numerical method for representing spatially variable sub-grid scale processes"; Advances in Water Resources, 31: 535-544. 


\section{ANEXOS}

\section{Documentos en Formato Digital}

Esta memoria es acompañada por un DVD que contiene toda la información recopilada y generada por esta tesis en formato digital. EI DVD ha sido organizado en carpetas como sigue:

Tabla 8.1: Listado de la documentación entregada en formato digital

\begin{tabular}{|l|ll|}
\hline CARPETA DEL DVD & \multicolumn{1}{c|}{ CONTENIDO } \\
\hline 01_Antecedentes consultados & - & Investigación bibliográfica por fecha \\
02_Programas y modelos utilizados & - & Investigación bibliográfica por autor \\
& - & Libros, Tesis y Material inédito \\
& - & Programas de GSLib en fortran 90 \\
03_Base de datos del Proyecto Cerro Solo & - & Programa SGems \\
& - & Programa W2p y rutina de optimización \\
04_Metodología desarrollada & - & Cartografía general y mapas de ubicación \\
& - & Ensayos de bombeo realizados \\
& - & Código del programa \\
& - & Ejemplos de casos sintéticos \\
& & Caso de estudio: ensayos de bombeo en el \\
& & distrito uranífero Cerro Solo \\
\hline
\end{tabular}

\section{Anexos incluidos en el presente texto}

A continuación, se agregan los siguientes anexos:

Tabla 8.2: Listado de los anexos incluidos en la tesis

\begin{tabular}{|c|c|}
\hline ANEXO & CONTENIDO \\
\hline 8.1_Anexo 1 & - Aplicación de la solución de De Smedt para acuíferos con doble porosidad. \\
8.2_Anexo 2 & - Ensayos de bombeo en el Distrito Cerro Solo, Chubut, Argentina. \\
8.3_Anexo 3 & - Archivos de Datos y programas auxiliares. \\
\hline
\end{tabular}




\subsection{APLICACIÓN DE LA SOLUCIÓN DE DE SMEDT PARA ACUÍfEROS CON DOBLE POROSIDAD}

Se presentan a continuación un par de casos de la práctica en los que se ha verificado el funcionamiento de la rutina de De Smedt (2011), comenzando por el sistema acuífero de Madison (Dakota del Sur, EEUU), reportado por Greene (1993), y siguiendo con el acuífero de Yuca Mountain (Nevada, EEUU) que fuera analizado por Moench (1984). Este último trabajo resulta de interés dado que en el mismo se hace referencia cierta a la influencia de una capa delgada de material de baja permeabilidad (fracture skin) que puede estar presente en la interfaz matriz-fractura.

\section{Acuífero de Madison}

Este ejemplo fue adoptado para poner a prueba la rutina de ajuste de la función $W_{2}(u, r / B, \varnothing)$ desarrollada por De Smedt (2011) y comparar sus resultados con el ajuste logrado por Greene (1999) para descensos observados a $549 \mathrm{~m}$ del pozo de bombeo. Los datos de partida son los siguientes:

Tabla 8.3: Datos del ensayo de bombeo del acuífero de Madison ( $\left.r_{0}=549 \mathrm{~m}\right)$

\begin{tabular}{rrrrrrrrrr}
\hline $\mathbf{t}[\mathbf{m i n}]$ & $\mathbf{d}[\mathbf{m}]$ & $\mathbf{t}[\mathbf{m i n}]$ & $\mathbf{d}[\mathbf{m}]$ & $\mathbf{t}[\mathbf{m i n}]$ & $\mathbf{d}[\mathbf{m}]$ & $\mathbf{t}[\mathbf{m i n}]$ & $\mathbf{d}[\mathbf{m}]$ & $\mathbf{t}[\mathbf{m i n}]$ & $\mathbf{d}[\mathbf{m}]$ \\
\hline 4 & 0,006 & 15 & 0,034 & 90 & 0,140 & 500 & 0,158 & 1500 & 0,213 \\
5 & 0,009 & 20 & 0,046 & 100 & 0,149 & 600 & 0,165 & 2000 & 0,238 \\
6 & 0,015 & 30 & 0,067 & 120 & 0,162 & 700 & 0,171 & 2910 & 0,241 \\
7 & 0,015 & 40 & 0,082 & 150 & 0,168 & 800 & 0,180 & 3340 & 0,302 \\
8 & 0,018 & 50 & 0,098 & 200 & 0,171 & 900 & 0,198 & 4470 & 0,302 \\
9 & 0,018 & 60 & 0,113 & 250 & 0,171 & 1000 & 0,195 & 5780 & 0,341 \\
10 & 0,021 & 70 & 0,125 & 300 & 0,168 & 1200 & 0,198 & 7450 & 0,457 \\
12 & 0,021 & 80 & 0,131 & 400 & 0,158 & 1370 & 0,213 & - & - \\
\hline
\end{tabular}

El caudal de bombeo de $3706 \mathrm{~m}^{3} / \mathrm{d}$, se mantuvo constante durante los 5 días del ensayo. La comparación de los ajustes de ambos métodos se muestra en la Figura 8.1.

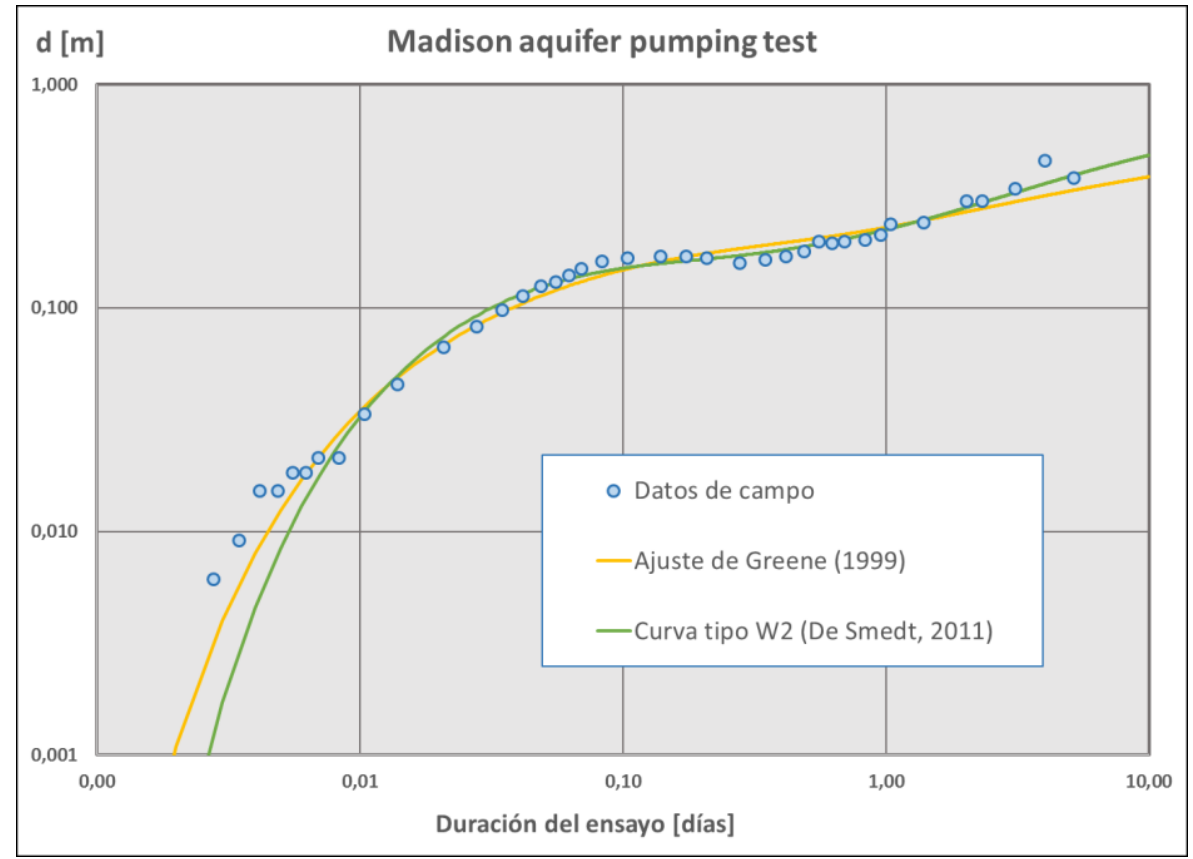

Figura 8.1: Ajustes de la función de pozo $W_{2}$ para el ensayo en el acuífero de Madison. 
La solución presentada en el capítulo 2, necesita de la estimación de 6 parámetros que definen el sistema de doble porosidad $\left(Q, r, T, S_{m}, S_{f}\right.$ y $\left.C\right)$. Su ajuste a las observaciones es bastante similar al logrado por Greene (1999) con las ecuaciones de flujo para un acuífero semiconfinado (Boulton et al., 1978), pero se puede comprobar que se desempeña levemente mejor en la zona de transición entre aporte neto de fracturas y de equilibrio con la componente matricial.

\section{Acuífero de Yuca Mountain}

En este caso, la metodología de ajuste es similar a la usada en el ejemplo anterior, sólo que ahora se introduce dentro del factor de forma $(\alpha)$ de la función de transferencia, el espesor del recubrimiento (fracture skin) en la zona de interfase y esto influye directamente los descensos observados que, en general, resultan mayores a los esperados con la curva teórica en la zona de transición. El pozo de observación estaba ubicado a $110 \mathrm{~m}$ del pozo de producción y el caudal bombeado fue de 3093,12 $\mathrm{m}^{3} /$ día. La duración del ensayo fue de 3 días.

En la Fig. 8.2 se muestra cómo, al aplicar la analogía de un medio poroso dual constituido por una sola familia de fracturas paralelas espaciadas $b^{\prime}=40 \mathrm{~m}$ (Moench, 1984), la curva tipo $W_{2}$ ajustada con los valores originales no alcanza a reproducir este efecto. En este trabajo se procedió a duplicar el espesor del recubrimiento con la intención de aumentar la resistencia a la cesión de agua procedente de la matriz ( $b^{\prime}$ es inversamente proporcional a $\alpha$ ) y, de esta forma, se puede advertir como mejora el ajuste con los datos experimentales.

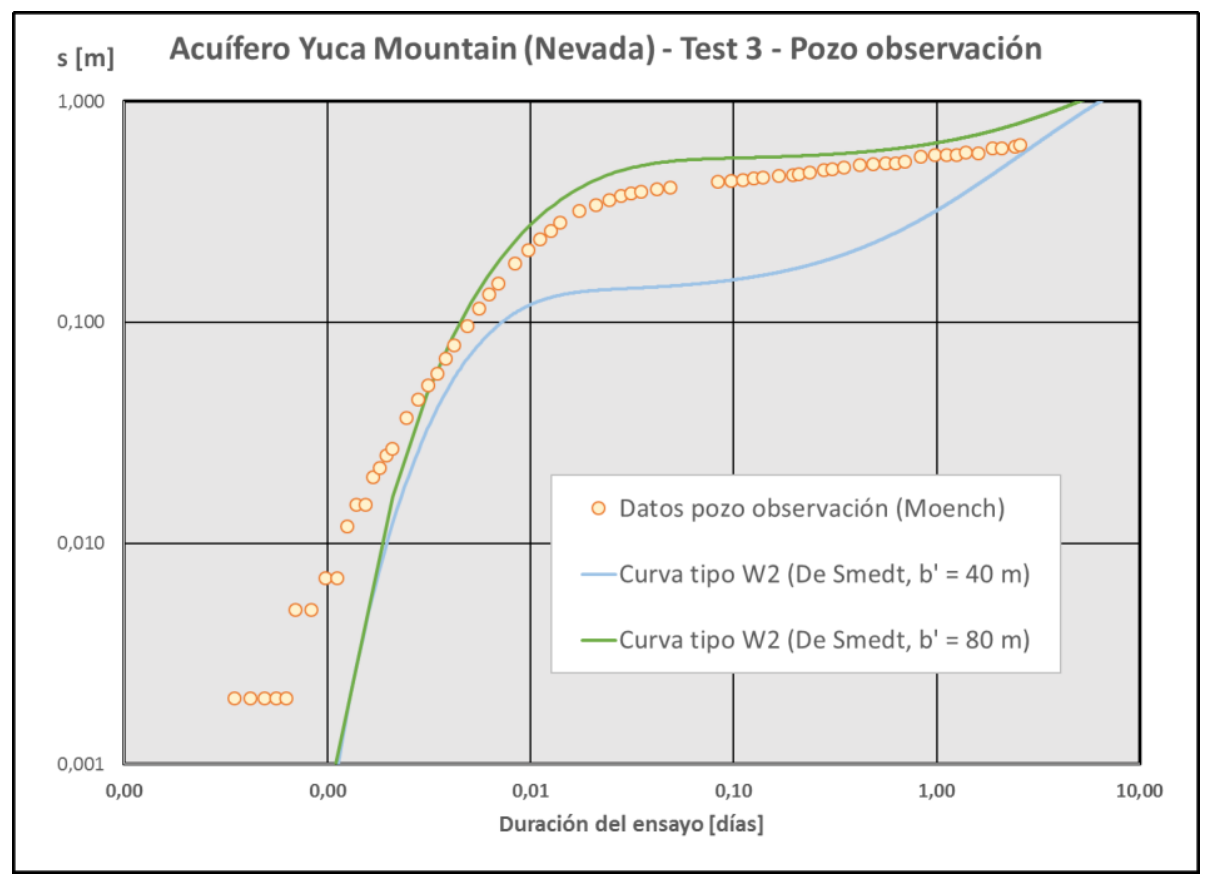

Figura 8.2: Ajustes de la función de pozo $W_{2}$ para el ensayo en el acuífero de Yuca Mountain.

Este efecto producido por la deficiencia de una descripción adecuada para la configuración geométrica de la familia de fracturas que atraviesan el acuífero de Yuca Mountain y, eventualmente, el efecto retardador del flujo de transferencia interna matriz-fractura producido por el relleno de las mismas, es lo que inspiró en este trabajo a tratar de conectar una forma expeditiva de generar estocásticamente la red de fisuras con los coeficientes de la FTMF. 


\subsection{Ensayos de bombeo en el Distrito Cerro Solo, Chubut, Argentina.}

Como se describiera en el capítulo 4 de esta tesis, existen dos unidades acuíferas en la región bajo estudio: una primera unidad de características freáticas denominada Post-cretácica y otra más profunda confinada (Unidad Cretácica acuífera), principal objetivo de este trabajo por su importancia. En esta última, se concretaron tanto la exploración por perforaciones como ensayos hidráulicos por bombeo. El objetivo de estos últimos fue el cálculo de los coeficientes de Conductividad, Trasmisividad y Almacenamiento de la Unidad Cretácica acuífera.

A continuación, se transcribe lo incluido en el informe realizado durante la campaña de ensayos (Hernández, Romanazzi, González y Trovatto, 2012):

"...El programa de ejecución de ensayos de bombeo incluyó dos tipos de prueba. Una primera breve a caudal variable, cuya finalidad fue estimar los caudales de bombeo más adecuados y las depresiones de nivel esperables en función de los descensos registrados, sirviendo también de optimización en la previa labor de limpieza y desarrollo de los sondeos.

Otro tipo fueron las de larga duración a caudal constante y recuperación, por métodos de noequilibrio, utilizando pozos de observación y complementariamente, de recuperación residual en el mismo pozo utilizado para extracción.

Para la resolución de los ensayos se recurrió al Programa Aquifer Test de la firma Waterloo, que permite, en base a una carga inicial única de datos, ingresar a los diferentes métodos según el tipo de información (por ejemplo, distancia al pozo de observación, espesor del acuitardo, valores de recuperación, etc.)....".

Por lo tanto, lo que se presenta en este apartado NO INCLUYE un análisis de resultados teniendo en cuenta la doble porosidad del medio acuífero naturalmente fracturado.

"...La interpretación de resultados del ensayo de bombeo en al menos un pozo de observación vecino se llevó a cabo utilizando los métodos de Theis y Cooper-Jacob, recuperación de Theis (sólo en el pozo de bombeo) y el de Papadópulos-Cooper (en un pozo de gran diámetro).

Se utilizaron pozos de observación construidos al efecto y además, en algunos casos, otros pozos mineros próximos, aunque como se verá sin buenos resultados. Los descensos en los pozos de observación específicos fueron registrados con sensores automáticos programables de nivel (divers) y accesoriamente con sonda electrónica de operación manual, en los pozos mineros adicionales.

Se emplearon bombas electrosumergibles de distinta potencia (30 HP y $10 \mathrm{HP}$ ) de acuerdo a los resultados de los ensayos de caudal variable, conduciéndose el agua desalojada a distancia para evitar su reingreso al sistema. Los caudales erogados fueron registrados con caudalímetro y por el método volumétrico (por desvío de manga a un tanque de 200 litros y conteo del tiempo de derivación).

En primer lugar, e independientemente de los pozos de exploración, se decidió reiterar dos test en los únicos sitios donde ya habían sido practicados oportunamente por la Comisión Nacional de Energía Atómica (CNEA, entidad a cargo de la exploración), denominados Ensayo 1 y Ensayo 2, bajo la metodología precedentemente descripta. También se incluyó un ensayo en una construcción de gran diámetro para un pique de labor minera, desactivado muchos años atrás y que por su estado y por alumbrar la Unidad Cretácica acuífera, reunía las condiciones para el propósito. 
En todos los casos, excepto para el sondeo de exploración EMB2, se utilizó un pozo de observación construido al efecto como ya se dijese. La oportunidad de contar con varias perforaciones mineras en las inmediaciones de EMB2 aconsejó no construir un piezómetro adicional...."

En las tablas 8.4 a 8.7 se presenta una síntesis de los resultados obtenidos.

Tabla 8.4: Resultados de los ensayos de bombeo en el yacimiento Cerro Solo

\begin{tabular}{|c|c|c|c|c|c|c|c|c|}
\hline & & \multicolumn{7}{|c|}{ Yacimiento Cerro Solo } \\
\hline & & \multicolumn{2}{|c|}{ CS } & \multicolumn{2}{|c|}{ Ensayo 2} & \multicolumn{2}{|c|}{ Ensayo 1} & \multirow{2}{*}{$\begin{array}{c}\begin{array}{c}\text { Pique } \\
\text { (gran D) }\end{array} \\
\begin{array}{c}\text { Pozo } \\
\text { Bombeo }\end{array}\end{array}$} \\
\hline & & $\begin{array}{l}\text { Pozo Bombeo } \\
(170,02 / 287,34)\end{array}$ & $\begin{array}{l}\text { Pozo Observ. } \\
(170,24 / 287,34)\end{array}$ & $\begin{array}{c}\text { Pozo } \\
\text { Bombeo } \\
(153,75 / 289)\end{array}$ & $\begin{array}{c}\text { Pozo Observ. } \\
(153,77 / 288,5)\end{array}$ & $\begin{array}{c}\text { Pozo Bombeo } \\
(151,5 / 293,5)\end{array}$ & $\begin{array}{c}\text { Pozo Observ. } \\
(151,25 / 293,5)\end{array}$ & \\
\hline \multicolumn{2}{|c|}{ Espesor saturado [m] } & 60 & 86 & 97,72 & 97,72 & 74,66 & 74,66 & - \\
\hline \multirow{2}{*}{ Recup. Theis } & $\mathrm{T}\left[\mathrm{m}^{2} / \mathrm{d}\right]$ & $4,12 \cdot 10^{-1}$ & \multirow{2}{*}{ - } & $6,11 \cdot 10^{2}$ & \multirow{2}{*}{ - } & $2,35.10^{2}$ & \multirow{2}{*}{ - } & \multirow{8}{*}{ - } \\
\hline & $\mathrm{K}[\mathrm{m} / \mathrm{d}]$ & $6,87 \cdot 10^{-3}$ & & $6,25.10^{\circ}$ & & $3,15.10^{\circ}$ & & \\
\hline \multirow{3}{*}{ Theis } & $\mathrm{T}\left[\mathrm{m}^{2} / \mathrm{d}\right]$ & \multirow{6}{*}{ - } & $9,76.10^{-1}$ & \multirow{6}{*}{ - } & $8,17.10^{2}$ & \multirow{6}{*}{ - } & $2,28.10^{2}$ & \\
\hline & $\mathrm{K}[\mathrm{m} / \mathrm{d}]$ & & $1,13.10^{-2}$ & & $8,37.10^{\circ}$ & & $3,06.10^{\circ}$ & \\
\hline & $S[-]$ & & $3,68.10^{-4}$ & & $2,52.10^{-5}$ & & $1,83.10^{-4}$ & \\
\hline \multirow{3}{*}{ Jacob } & $\mathrm{T}\left[\mathrm{m}^{2} / \mathrm{d}\right]$ & & $1,54.10^{\circ}$ & & $7,34.10^{2}$ & & $2,18.10^{2}$ & \\
\hline & $\mathrm{K}[\mathrm{m} / \mathrm{d}]$ & & $1,79.10^{-2}$ & & $7,51.10^{\circ}$ & & $2,93.10^{\circ}$ & \\
\hline & $S[-]$ & & $2,49.10^{-4}$ & & $4,33.10^{-5}$ & & $1,60.10^{-4}$ & \\
\hline \multirow{2}{*}{$\begin{array}{c}\text { Papadópulos } \\
- \\
\text { Cooper }\end{array}$} & $\mathrm{T}\left[\mathrm{m}^{2} / \mathrm{d}\right]$ & & & & & \multirow{2}{*}{\multicolumn{2}{|c|}{ - }} & $1,20.10^{1}$ \\
\hline & $S[-]$ & & & & & & & $8.10^{-2}$ \\
\hline
\end{tabular}

Tabla 8.5: Resultados de los ensayos de bombeo en Puesto Alvear y El Ganso.

\begin{tabular}{|c|c|c|c|c|c|c|c|}
\hline & & \multicolumn{3}{|c|}{ Puesto Alvear } & \multicolumn{3}{|c|}{ El Ganso } \\
\hline & & $\begin{array}{c}\text { PAB } \\
(169,96 / 309,81)\end{array}$ & $\begin{array}{c}\text { PAO } \\
(169,66 / 309,86)\end{array}$ & $\begin{array}{c}\text { POCNEA } \\
(170 / 310)\end{array}$ & $\begin{array}{c}\text { EGB } \\
(135,04 / 245,22)\end{array}$ & $\begin{array}{c}\text { EGO } \\
(134,78 / 245,38)\end{array}$ & POCNEA \\
\hline \multicolumn{2}{|c|}{ Espesor saturado $[\mathrm{m}]$} & 98 & 98 & 98 & 50 & 52 & - \\
\hline \multirow{2}{*}{$\begin{array}{l}\text { Recup. } \\
\text { Theis }\end{array}$} & $\mathrm{T}\left[\mathrm{m}^{2} / \mathrm{d}\right]$ & $1,70.10^{1}$ & - & - & $2,44.10^{2}$ & - & \multirow{8}{*}{$\begin{array}{l}0 \\
0 \\
0 \\
0 \\
0 \\
0 \\
0 \\
3 \\
3 \\
0 \\
0 \\
0\end{array}$} \\
\hline & $\mathrm{K}[\mathrm{m} / \mathrm{d}]$ & $1,74.10^{-1}$ & - & - & $4,69.10^{\circ}$ & - & \\
\hline \multirow{3}{*}{ Theis } & $\mathrm{T}\left[\mathrm{m}^{2} / \mathrm{d}\right]$ & - & $9,86 \cdot 10^{-1}$ & $1,79.10^{2}$ & - & $7,53.10^{1}$ & \\
\hline & $\mathrm{K}[\mathrm{m} / \mathrm{d}]$ & - & $1,00 \cdot 10^{-2}$ & $1,82.10^{\circ}$ & - & $1,50.10^{\circ}$ & \\
\hline & $\mathrm{S}[-]$ & - & $3,34.10^{-6}$ & $3,47.10^{-5}$ & - & $6,60 \cdot 10^{-4}$ & \\
\hline \multirow{3}{*}{ Jacob } & $\mathrm{T}\left[\mathrm{m}^{2} / \mathrm{d}\right]$ & - & $1,18.10^{\circ}$ & $1,68.10^{2}$ & - & $9,40.10^{1}$ & \\
\hline & $\mathrm{K}[\mathrm{m} / \mathrm{d}]$ & - & $1,21.10^{-2}$ & $1,71.10^{\circ}$ & - & $1,88.10^{\circ}$ & \\
\hline & $S[-]$ & - & $2,27.10^{-6}$ & $2,93.10^{-5}$ & - & $5,83.10^{-4}$ & \\
\hline
\end{tabular}


Tabla 8.6: Resultados de los ensayos de bombeo en El Molino y Arroyo Perdido.

\begin{tabular}{|c|c|c|c|c|c|c|c|c|c|c|}
\hline & & \multicolumn{7}{|c|}{ EI Molino } & \multirow{2}{*}{\multicolumn{2}{|c|}{$\begin{array}{c}A^{\circ} \text { Perdido } \\
\text { APB }\end{array}$}} \\
\hline & & \multicolumn{4}{|c|}{ EMB2 } & \multicolumn{3}{|c|}{ EMB1 } & & \\
\hline & & $\begin{array}{r}\text { EMB2 } \\
(150 / 428)\end{array}$ & $\begin{array}{c}\text { PO1 } \\
(150 / 430)\end{array}$ & $\mathrm{PO} 2$ & $\begin{array}{c}\text { PO3 } \\
(150 / 425)\end{array}$ & $\begin{array}{c}\text { EMB1 } \\
(140,18 / 430,25)\end{array}$ & $\begin{array}{c}\text { PO1 } \\
(139,98 / 430,40)\end{array}$ & $\begin{array}{l}\text { PO2 } \\
(140 / 430)\end{array}$ & $\begin{array}{c}\text { APB } \\
(120,25 / 480)\end{array}$ & $\begin{array}{c}\text { APO } \\
(120,45 / 480)\end{array}$ \\
\hline \multicolumn{2}{|c|}{ Espesor saturado [m] } & 52 & 52 & 52 & 52 & 60 & 60 & 60 & 66 & 75 \\
\hline \multirow{2}{*}{$\begin{array}{l}\text { Recup. } \\
\text { Theis }\end{array}$} & $\mathrm{T}\left[\mathrm{m}^{2} / \mathrm{d}\right]$ & $4,45.10^{=}$ & - & \multirow{8}{*}{$\begin{array}{l}0 \\
0 \\
0 \\
0 \\
0 \\
0 \\
0 \\
\vdots 3 \\
3 \\
0 \\
0 \\
0 \\
0 \\
0 \\
0 \\
0 \\
0 \\
0\end{array}$} & - & $4,02 \cdot 10^{-1}$ & - & - & $3,02 \cdot 10^{-1}$ & - \\
\hline & $\mathrm{K}[\mathrm{m} / \mathrm{d}]$ & $8,56.10^{-2}$ & - & & - & $6,70.10^{-3}$ & - & - & $4,57.10^{-3}$ & - \\
\hline \multirow{3}{*}{ Theis } & $\mathrm{T}\left[\mathrm{m}^{2} / \mathrm{d}\right]$ & - & $2,55.10^{1}$ & & $2,07.10^{\circ}$ & - & $8,17 \cdot 10^{-1}$ & $4,77.10^{\circ}$ & - & $3,17 \cdot 10^{-1}$ \\
\hline & $K[\mathrm{~m} / \mathrm{d}]$ & - & $4,91 \cdot 10^{-1}$ & & $3,99 \cdot 10^{-2}$ & - & $1,36.10^{-2}$ & $7,95.10^{-2}$ & - & $4,23.10^{-3}$ \\
\hline & $S[-]$ & - & $5,15 \cdot 10^{-4}$ & & $4,31.10^{-5}$ & - & $2,74.10^{-5}$ & $5,78.10^{-4}$ & - & $3,43 \cdot 10^{-5}$ \\
\hline \multirow{3}{*}{ Jacob } & $\mathrm{T}\left[\mathrm{m}^{2} / \mathrm{d}\right]$ & - & $1,33.10^{1}$ & & $4,99.10^{\circ}$ & - & $1,05.10^{\circ}$ & $6,0.10^{\circ}$ & - & $4,25.10^{-1}$ \\
\hline & $\mathrm{K}[\mathrm{m} / \mathrm{d}]$ & - & $2,56.10^{-1}$ & & $9,59 \cdot 10^{-2}$ & - & $1,75.10^{-2}$ & $1,00.10^{-1}$ & - & $5,66.10^{-3}$ \\
\hline & $S[-]$ & - & $3,49.10^{-4}$ & & $3,32 \cdot 10^{-5}$ & - & $1,73.10^{-5}$ & $3,78.10^{-4}$ & - & $2,04.10^{-5}$ \\
\hline
\end{tabular}

Tabla 8.7: Distancias calculadas entre pozos de bombeo y de observación.

\begin{tabular}{|c|c|c|c|c|c|c|c|}
\hline YACIMIENTO & SECTOR & SONDEO DE BOMBEO & Xbombeo & Ybombeo & № DE MUESTREO & SONDEO DE OBSERVACIÓN & DISTANCIA X-Y (m) \\
\hline \multirow{35}{*}{ Cerro Solo } & \multirow{12}{*}{$\begin{array}{c}\text { Pozo Ensayo } \\
\text { 2(B) }\end{array}$} & \multirow{12}{*}{$153,750 / 289,000$} & \multirow{12}{*}{15374} & \multirow{12}{*}{28900} & 1 & $153,770 / 288,750$ & 24,94 \\
\hline & & & & & 2 & $154,000 / 288,500$ & 56,36 \\
\hline & & & & & 3 & $154,250 / 289,000$ & 50,92 \\
\hline & & & & & 4 & $154,250 / 289,250$ & 56,52 \\
\hline & & & & & 5 & $154,250 / 289,500$ & 71,47 \\
\hline & & & & & 6 & $153,750 / 289,250$ & 25,07 \\
\hline & & & & & 7 & $153,500 / 289,250$ & 35,55 \\
\hline & & & & & 8 & $153,250 / 289,000$ & 49,12 \\
\hline & & & & & 9 & $153,500 / 288,750$ & 34,49 \\
\hline & & & & & 10 & $153,750 / 288,500$ & 49,60 \\
\hline & & & & & 11 & $153,750 / 288,250$ & 74,46 \\
\hline & & & & & 12 & $153,750 / 289,500$ & 50,00 \\
\hline & \multirow{18}{*}{$\begin{array}{c}\text { Pozo Ensayo } \\
1(\mathrm{C})\end{array}$} & \multirow{18}{*}{$151,500 / 293,500$} & \multirow{18}{*}{15150} & \multirow{18}{*}{29350} & 1 & $151,250 / 293,500$ & 24,84 \\
\hline & & & & & 2 & $151,000 / 293,000$ & 69,31 \\
\hline & & & & & 3 & $151,500 / 293,000$ & 51,00 \\
\hline & & & & & 4 & $151,750 / 293,250$ * & 35,13 \\
\hline & & & & & 5 & $151,750 / 293,500$ & 25,08 \\
\hline & & & & & 6 & $151,500 / 293,750$ & 25,13 \\
\hline & & & & & 7 & $151,250 / 293,750$ & 35,22 \\
\hline & & & & & 8 & $150,480 / 293,750$ & 104,53 \\
\hline & & & & & 9 & $150,500 / 293,250$ & 103,28 \\
\hline & & & & & 10 & $150,750 / 292,750$ & 105,84 \\
\hline & & & & & 11 & $151,250 / 292,500$ & 102,64 \\
\hline & & & & & 12 & $151,750 / 292,500$ & 102,88 \\
\hline & & & & & 13 & $152,250 / 293,000$ & 90,13 \\
\hline & & & & & 14 & $152,250 / 293,750$ & 79,22 \\
\hline & & & & & 15 & $152,000 / 294,250$ & 90,54 \\
\hline & & & & & 16 & $151,250 / 294,250$ & 78,69 \\
\hline & & & & & 17 & $151,000 / 294,000$ & 70,72 \\
\hline & & & & & 18 & $151,000 / 293,500$ & 50,01 \\
\hline & \multirow{3}{*}{ IX-14 } & \multirow{3}{*}{ PIQUE } & \multirow{3}{*}{15879,25} & \multirow{3}{*}{29371,43} & 1 & $158,750 / 294,000^{*}$ & 25,12 \\
\hline & & & & & 2 & $158,000 / 293,200$ & 95,45 \\
\hline & & & & & 3 & $158,200 / 292,450$ & 137,88 \\
\hline & \multirow{2}{*}{ Graben } & \multirow{2}{*}{$170,020 / 287,340$} & \multirow{2}{*}{16996,58} & \multirow{2}{*}{28735,99} & & $170,240 / 287,290$ & 32,88 \\
\hline & & & & & & $170,000 / 287,000$ & 28,11 \\
\hline \multirow[t]{2}{*}{ Puesto Alvear } & & \multirow{2}{*}{$169,960 / 309,810$} & \multirow{2}{*}{16996,151} & 30981003 & & $169,660 / 309,860$ & 30,22 \\
\hline & & & & 30981,003 & & $170,000 / 310,000 \mathrm{~b}$ & 19,56 \\
\hline & & & & & 1 & $134,780 / 245,380$ & 30,20 \\
\hline FlGanso & & $125010 / 245020$ & 12504382 & 24522672 & 2 & $135,000 / 245,000 \mathrm{c}$ & 20,03 \\
\hline El Ganso & & $135,040 / 245,220$ & 13504,383 & $24522,6 / 2$ & 3 & $140,000 / 255,000$ & 1095,57 \\
\hline & & & & & 4 & $130,000 / 240,000$ & 724,09 \\
\hline & 1 & $140180 / 430-250$ & 14018127 & 43024674 & & $139,980 / 430,400$ & 24,07 \\
\hline & 1 & & & 43024,674 & & $140,000 / 430,000$ & 30,02 \\
\hline ElMoling & & & & & 1 & $150,000 / 430,000$ & 200,35 \\
\hline & 2 & $150000 / 428000$ & 15000227 & 42800616 & 2 & $151,000 / 427,000^{*}$ & 141,82 \\
\hline & 2 & $150,000 / 428,000$ & 15000,227 & 42800,616 & 3 & $150,000 / 425,000$ & 300,62 \\
\hline & & & & & & $147,000 / 425,000$ & 415,68 \\
\hline Arroyo Perdido & & $120,250 / 480,000$ & 12025,351 & 47998,644 & & $120,450 / 480,000$ bis & 16,83 \\
\hline $\mathrm{ob}$ & eda $\sin a$ & rante bombeo & & & & & \\
\hline
\end{tabular}


A continuación, se presentan las curvas de descenso y de recuperación en los sitios ensayados. En cada figura se indica el nivel inicial y los descensos tanto en el pozo de bombeo como en los pozos de observación vecinos.

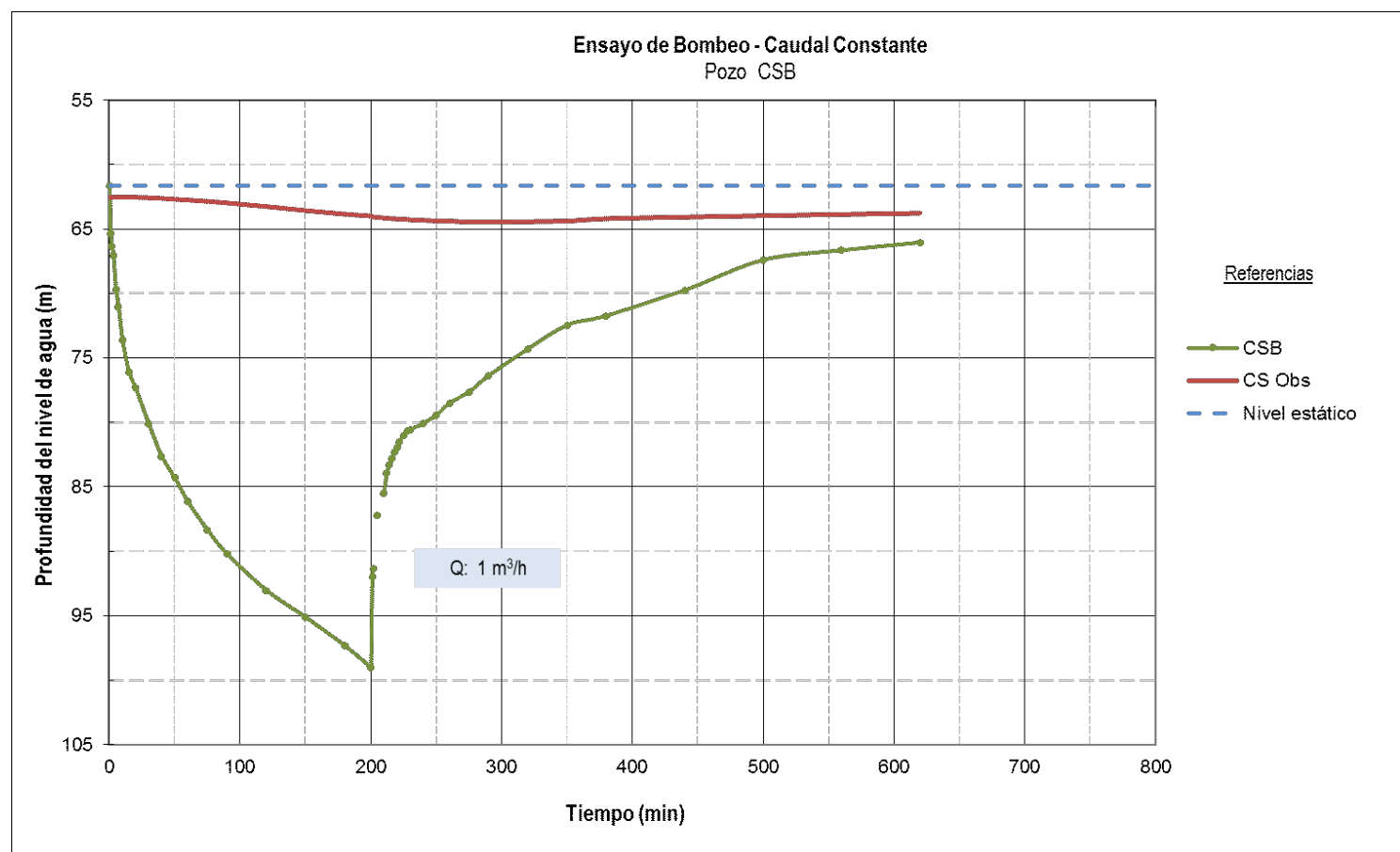

Figura 8.3: Ensayo de bombeo en Sitio Cerro Solo.

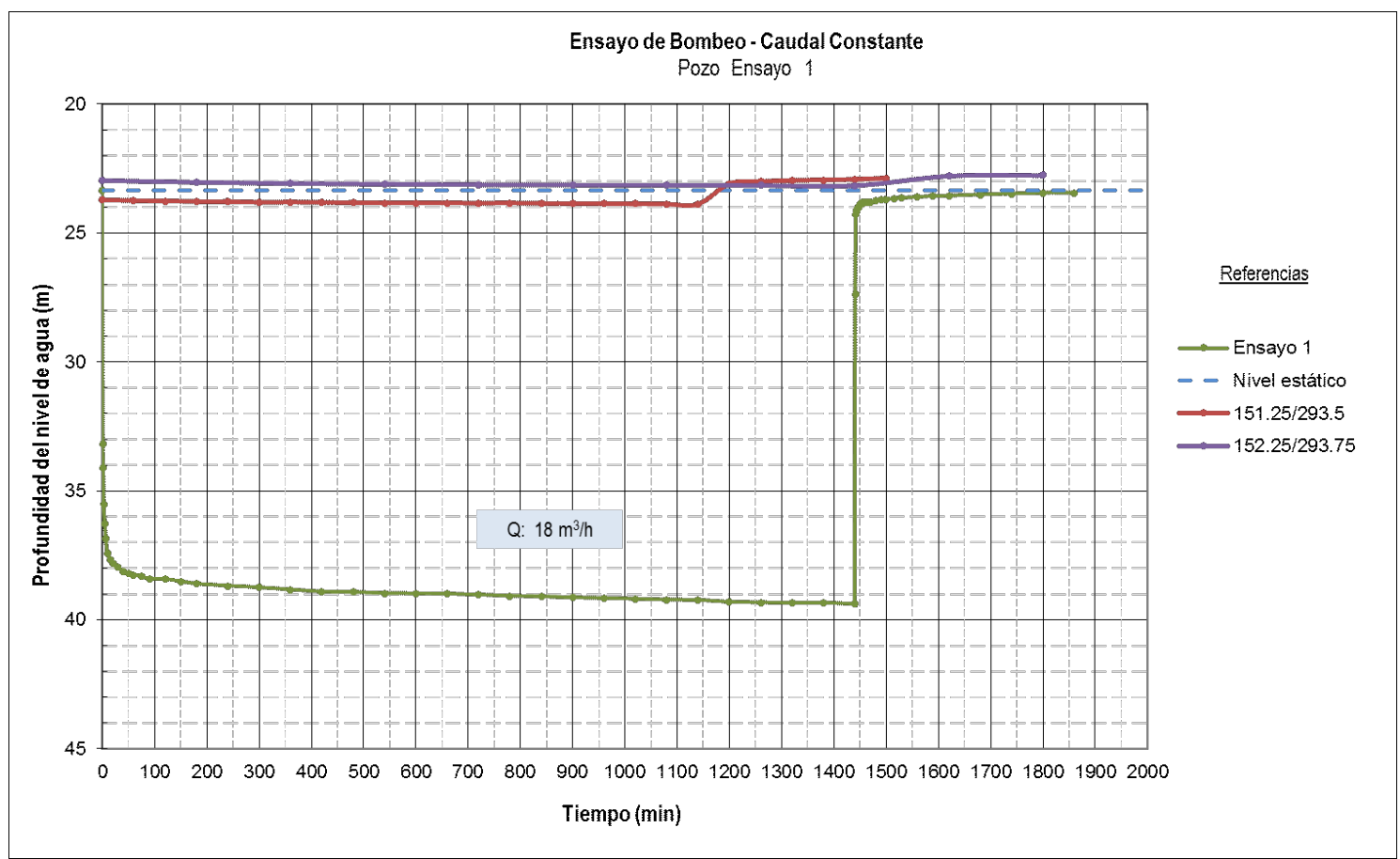

Figura 8.4: Ensayo de bombeo en el Pozo Ensayo 1 (Yacimiento Cerro Solo). 


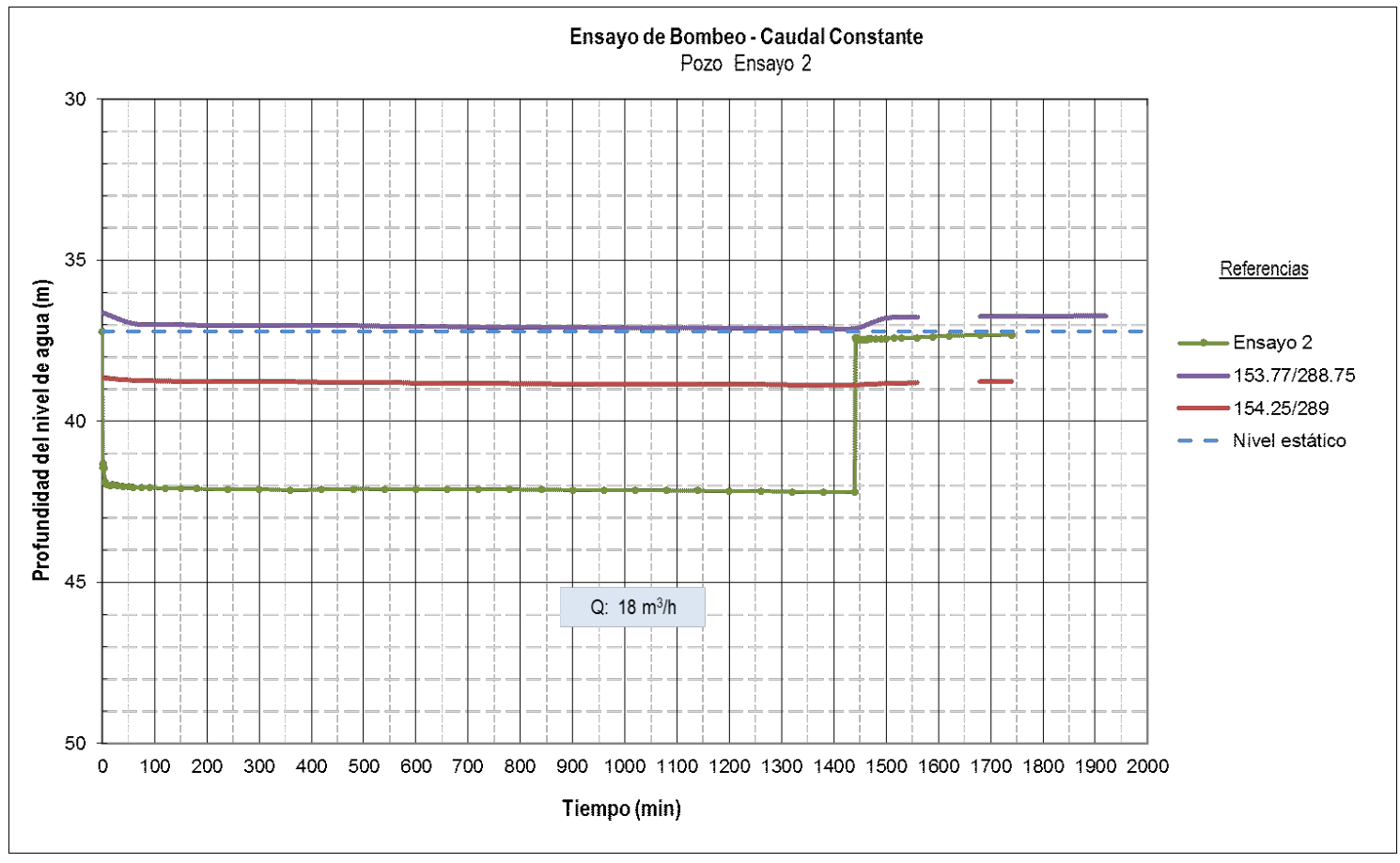

Figura 8.5: Ensayo de bombeo en el Pozo Ensayo 2 (Yacimiento Cerro Solo).

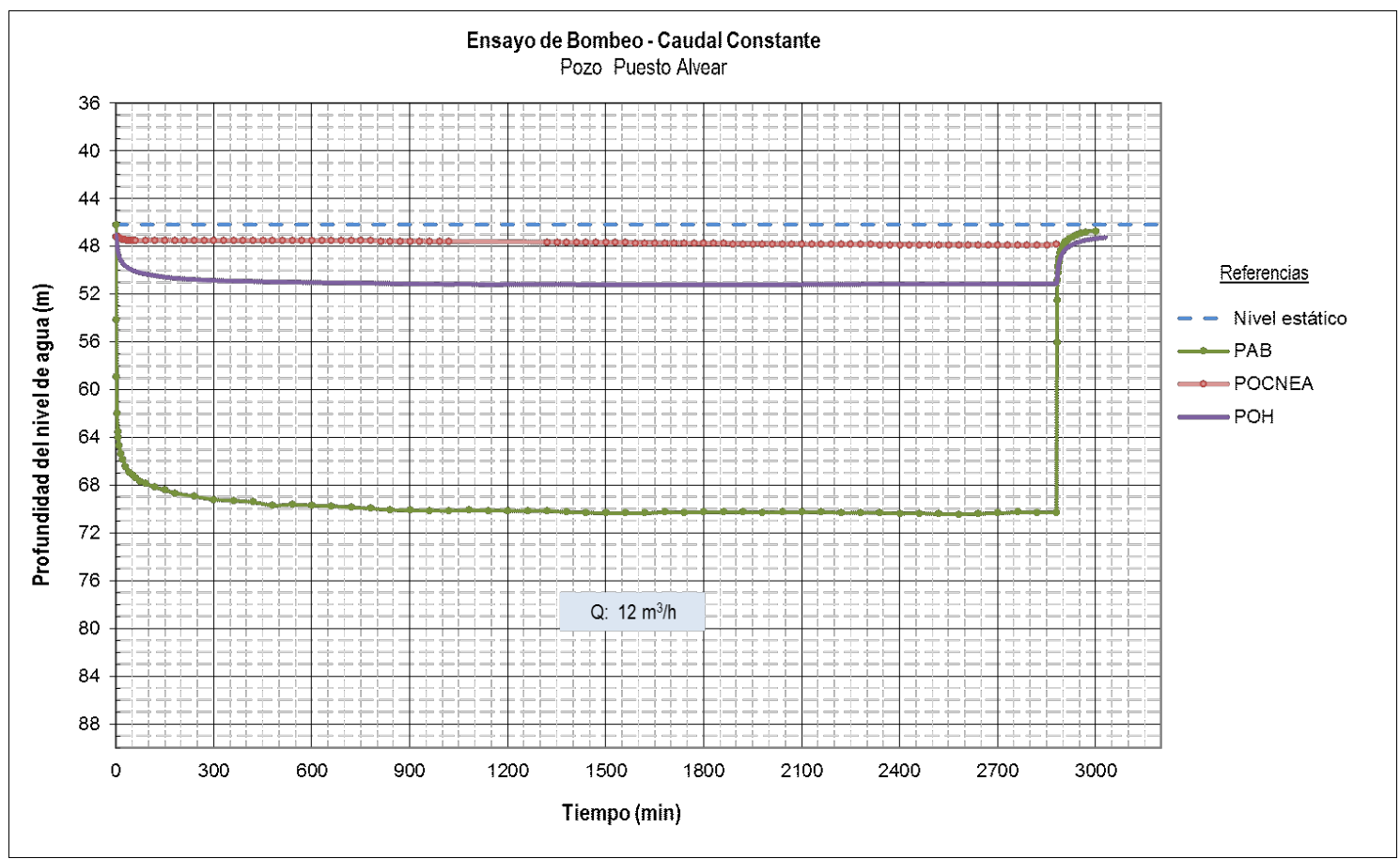

Figura 8.6: Ensayo de bombeo en el sitio Puesto Alvear. 


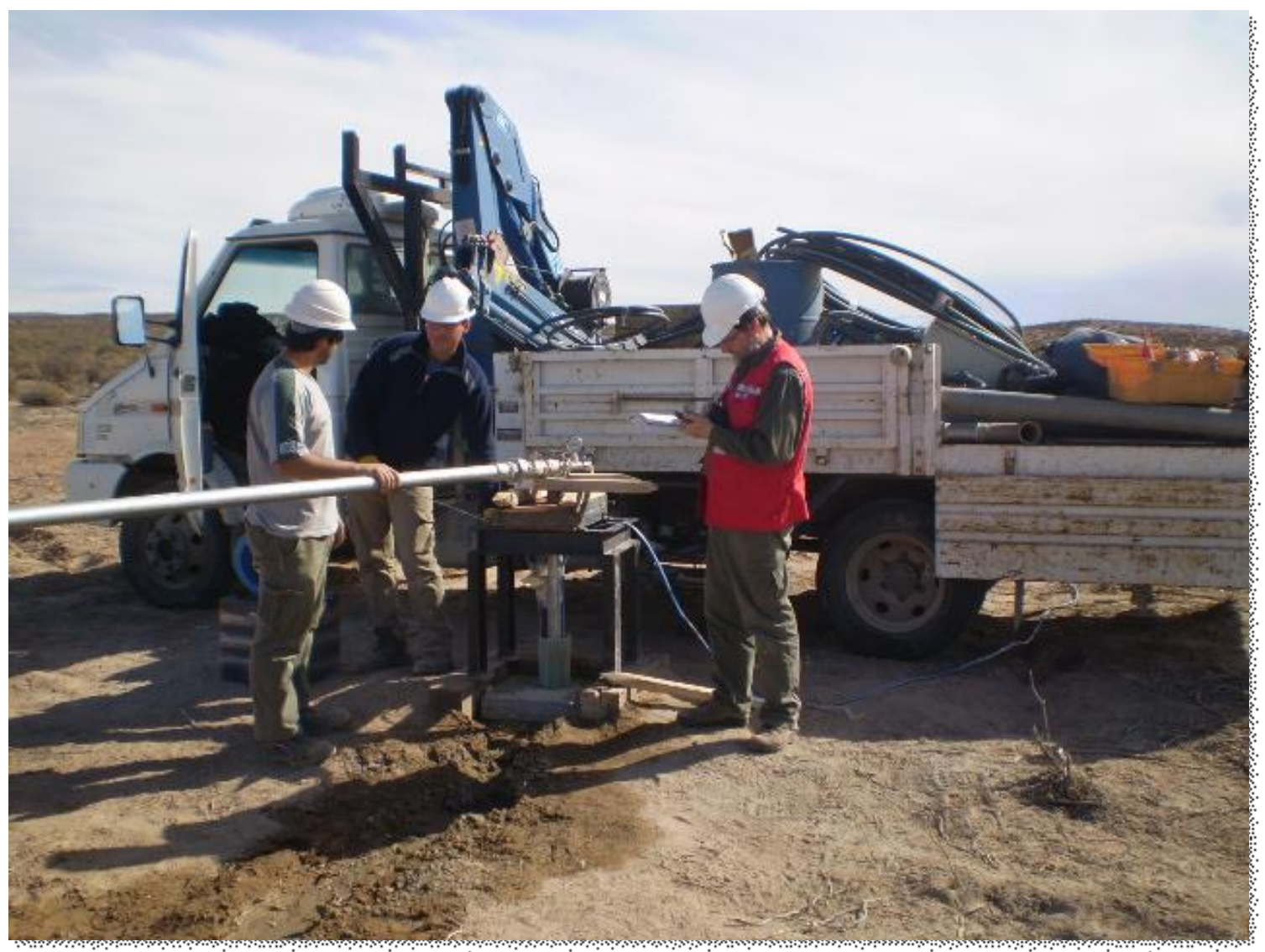

Foto 8.1: Ensayo de bombeo en Puesto Alvear.

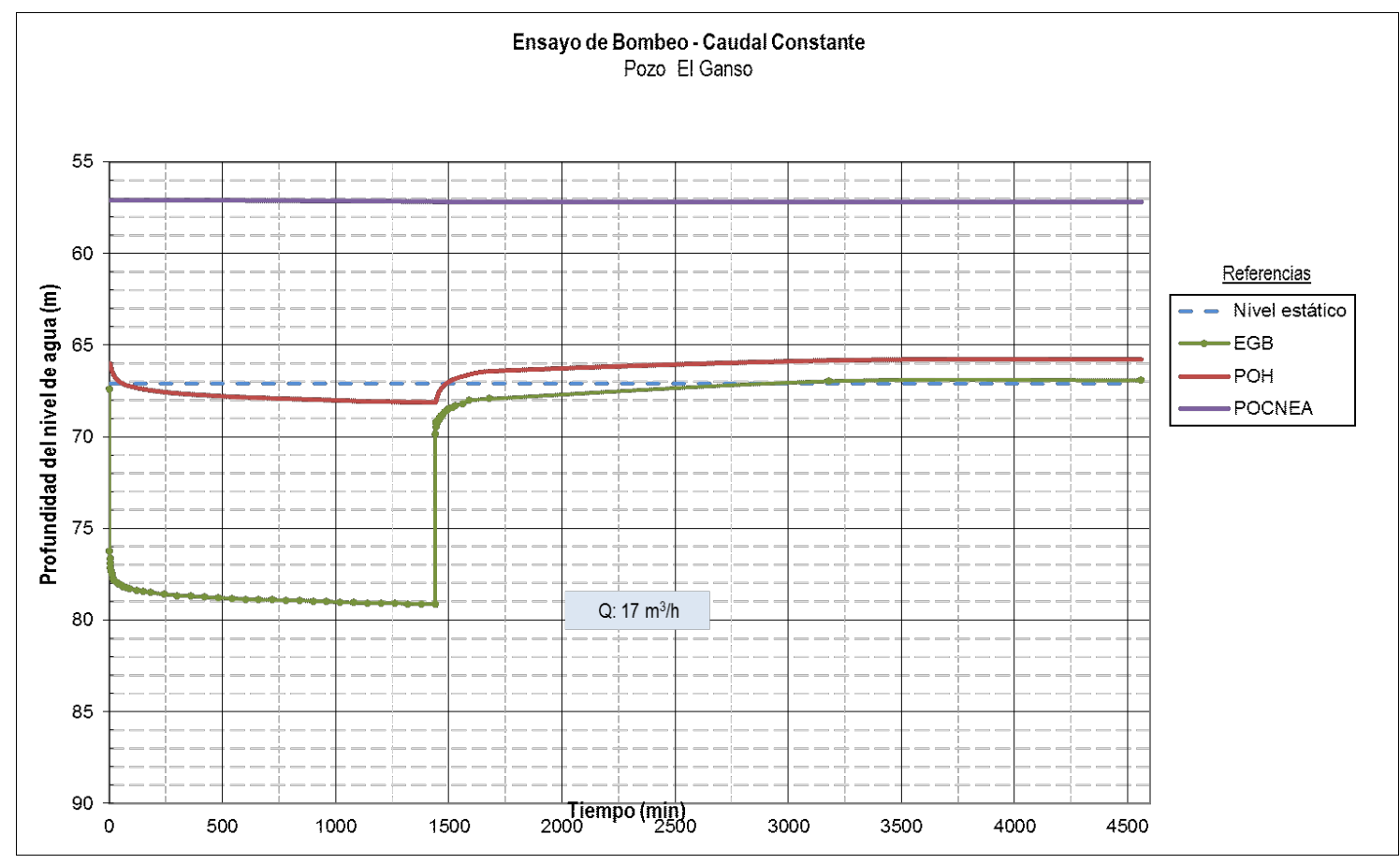

Figura 8.7: Ensayo de bombeo en el sitio El Ganso. 


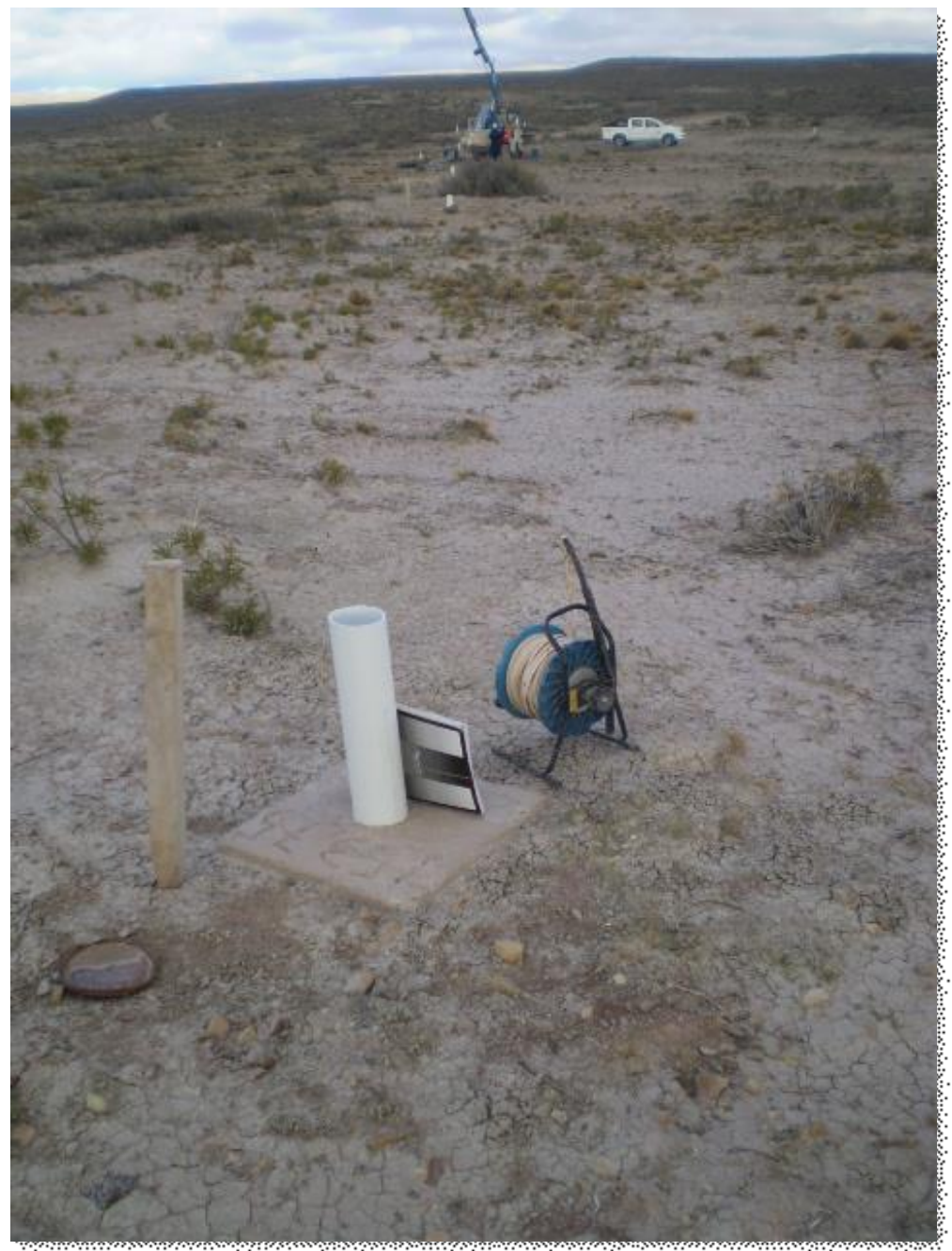

Foto 8.2: Ensayo de bombeo en El Molino (1).

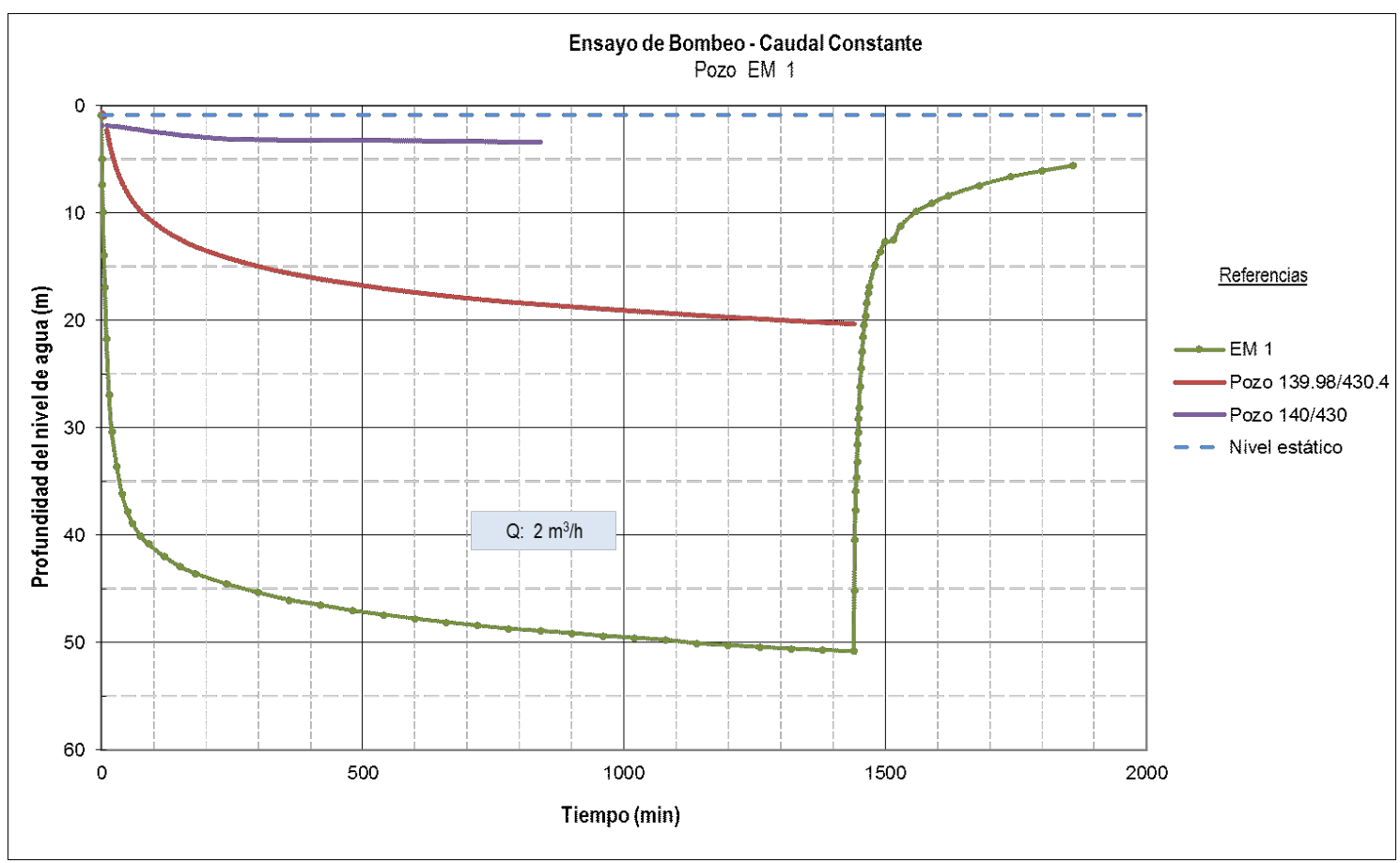

Figura 8.8: Ensayo de bombeo en el sitio El Molino (1). 


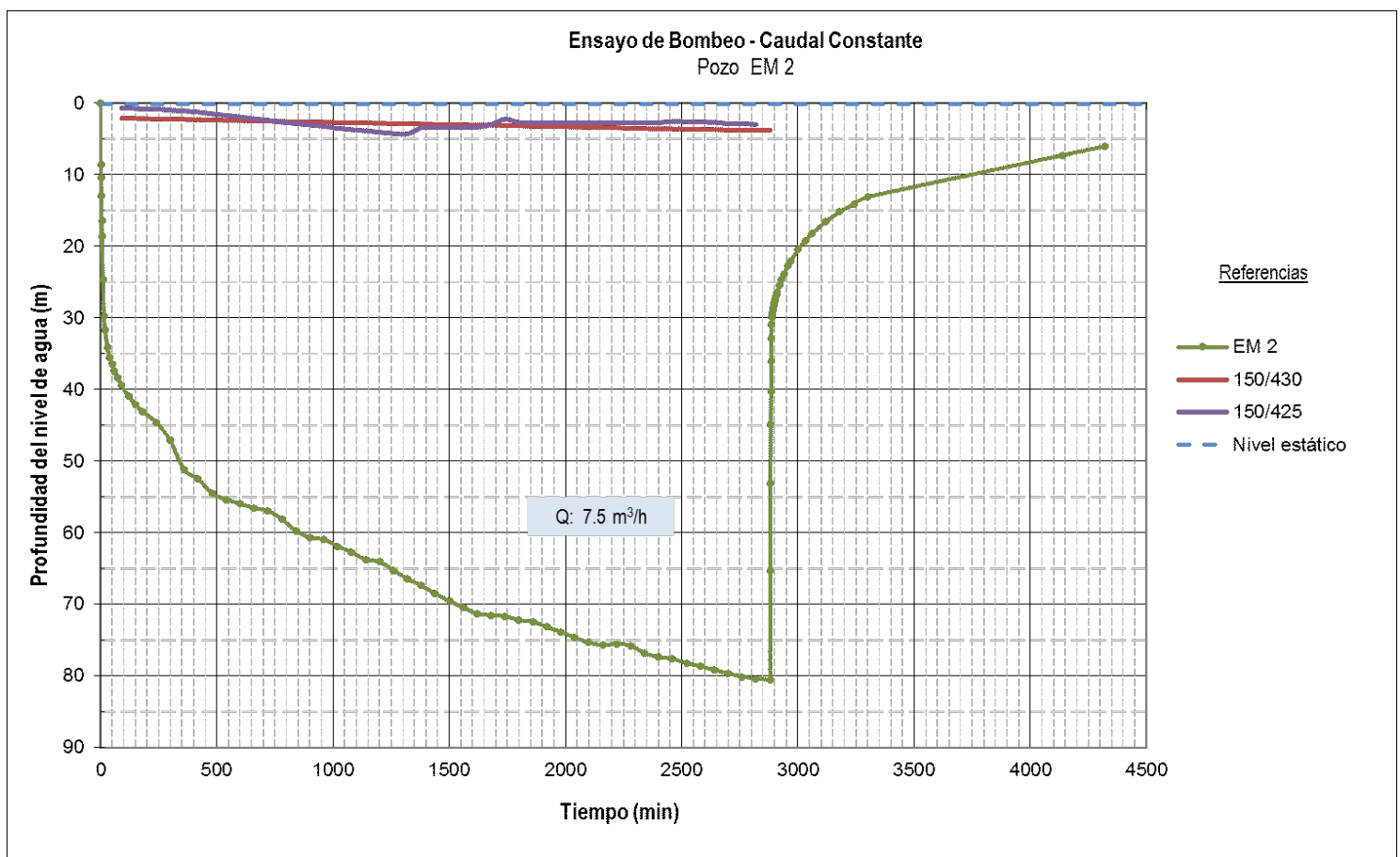

Figura 8.9: Ensayo de bombeo en el sitio El Molino (2).

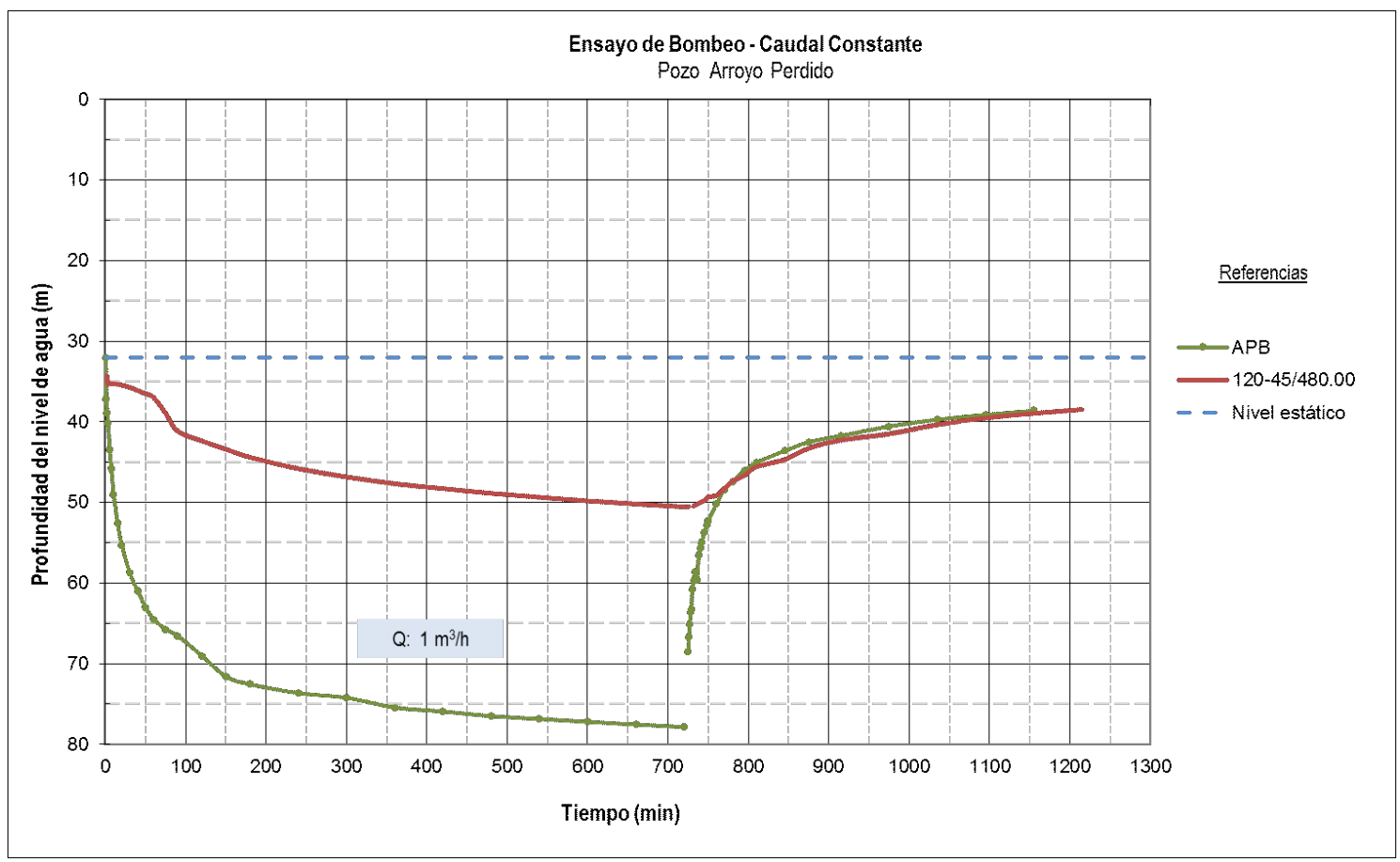

Figura 8.10: Ensayo de bombeo en el sitio Arroyo Perdido. 


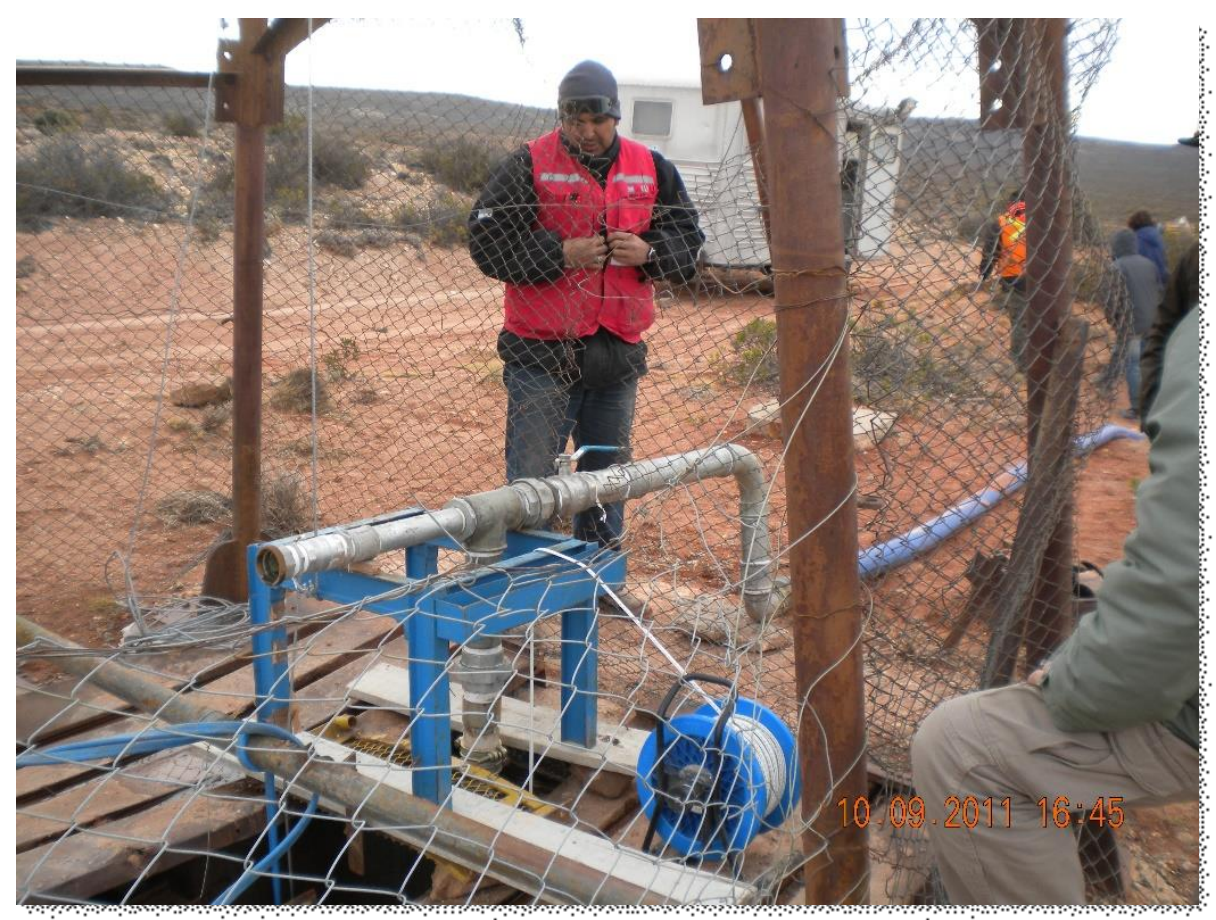

Foto 8.3: Ensayo de bombeo en El Pique (pozo de gran diámetro).

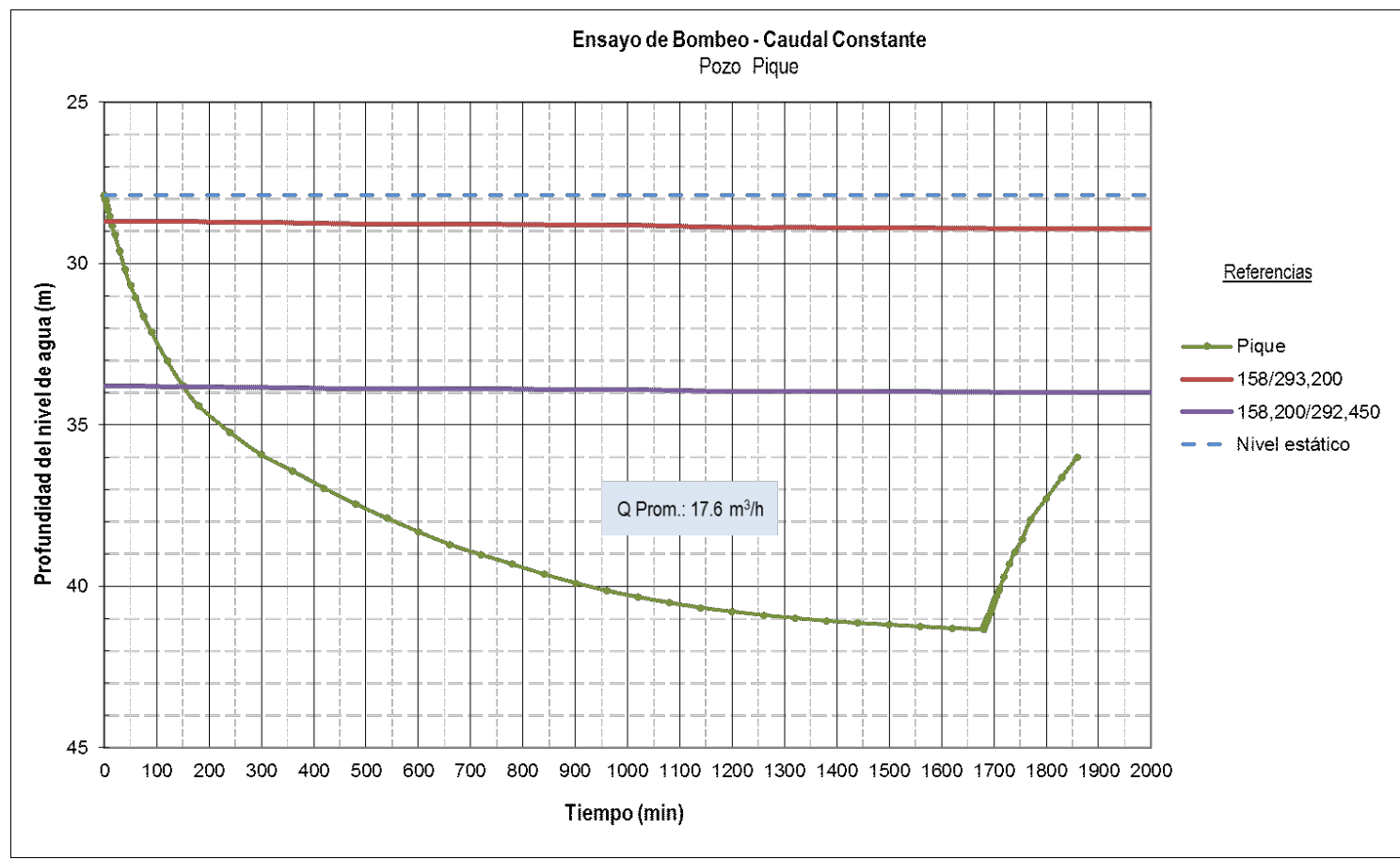

Figura 8.11: Ensayo de bombeo en el sitio El Pique (gran diámetro).

“...Del total de test realizados, no fueron satisfactorios los practicados en los pozos de exploración Cerro Solo (CSB y CSO), probablemente por no haberse podido desarrollarlos adecuadamente, ya que luego de varios intentos de ensayo no dejó de erogarse agua con material fino y los caudales característicos resultaron escasos, con eficiencia de pozo también baja. 
Excepto en Puesto Alvear y El Molino, tampoco resultaron mayormente útiles los pozos mineros existentes cuando se los utilizó a modo de observación, lo cual se explica por no haber sido diseñados para esa finalidad y además, por el tiempo transcurrido desde su construcción en la mayor parte de los casos.

Del resto de las pruebas, las mejores logradas fueron las correspondientes a Cerro Solo (Ensayos 1, 2 y Pique), Puesto Alvear y El Ganso. Tanto en EI Molino como en Arroyo Perdido, los caudales ofrecidos por el acuífero fueron relativamente escasos y hubo necesidad de regularlos en valores bajos para compensar las depresiones excesivas y garantizar la duración de los ensayos.

También debe tenerse en cuenta la ya mencionada anisotropía y heterogeneidad del acuífero confinado, a la cual se une la existencia de doble porosidad irregularmente manifiesta, como ya se hizo referencia.

Los espesores saturados ensayados fueron representativos de la unidad, ya que superaron en todos los casos los $50 \mathrm{~m}$ y alcanzaron casi a $100 \mathrm{~m}$. No se efectuaron correcciones por penetración parcial, dadas las mencionadas anisotropía y heterogeneidad.

Los valores del coeficiente de Trasmisividad (T) calculados más acordes con la hidrolitología percibida a través de los pozos de exploración y los espesores registrados, son los de los Ensayos 1, 2 y Pique, situados en el mismo orden de magnitud por los diferentes métodos aplicados. Para el Ensayo 2 por ejemplo, $T$ toma valores de $611 \mathrm{~m}^{2} / \mathrm{d}, 817 \mathrm{~m}^{2} / \mathrm{d}$ y $734 \mathrm{~m}^{2} / d$ según Recuperación de Theis, Theis y Cooper-Jacob respectivamente. En el ensayo 1 en el mismo orden, $235 \mathrm{~m}^{2} / \mathrm{d}, 228 \mathrm{~m}^{2} / \mathrm{d}$ y $218 \mathrm{~m}^{2} / \mathrm{d}$.

Para Puesto Alvear en un pozo de observación se obtienen $179 \mathrm{~m}^{2} / \mathrm{d}$ y $168 \mathrm{~m}^{2} / \mathrm{d}$, mientras que en el de bombeo por Recuperación de Theis solamente $17 \mathrm{~m}^{2} / d$. En El Ganso, contra $244 \mathrm{~m}^{2} / d$ por Recuperación de Theis, se logran valores próximos a $100 \mathrm{~m}^{2} / \mathrm{d}\left(75 \mathrm{~m}^{2} / \mathrm{d}\right.$ y $\left.94 \mathrm{~m}^{2} / \mathrm{d}\right)$ por los otros dos métodos. Hasta aquí, aparecen las magnitudes mayores y más coherentes con el tipo hidrolitológico, del orden de 100 a $800 \mathrm{~m}^{2} / \mathrm{d}$.

En los otros dos sectores se hallan trasmisividades francamente menores, del orden de 4,5 $\mathrm{m}^{2} / \mathrm{d}, 25 \mathrm{~m}^{2} / \mathrm{d}$ y $13 \mathrm{~m}^{2} / \mathrm{d}$ (EMB2) con los métodos según se los ha venido citando, y de $0,4 \mathrm{~m}^{2} / \mathrm{d}$ a $6 \mathrm{~m}^{2} / d$ en EMB1. Finalmente, en Arroyo Perdido toman valores de $0,3 \mathrm{~m}^{2} / \mathrm{d}$ a $0,4 \mathrm{~m}^{2} / \mathrm{d}$. Independientemente de los condicionantes hidrolitológicos y la doble porosidad, puede verse una disminución franca hacia el Este, coincidentemente con menores espesores y fundamentalmente, litología más fina.

Respecto al Coeficiente de Conductividad (K), que abstrae por definición el factor espesor saturado, en los Ensayos 1 y 2 de Cerro Solo, Puesto Alvear y El Ganso se obtuvieron cifras propias de sedimentos como los indicados en los perfiles de los pozos de exploración: $3 \mathrm{~m} / \mathrm{d}$ a 8,4 m/d en Cerro Solo, 0,17 m/d a 1,8 m/d en Puesto Alvear y 1,5 m/d a 4,7 m/d en El Ganso. Menores son los brindados por los ensayos en EI Molino $(0,01 \mathrm{~m} / \mathrm{d}$ a 0,09 $\mathrm{m} / \mathrm{d}$ como valores más frecuentes) y Arroyo Perdido $(0,004$ a 0,006 m/d). Además de la influencia de la anisotropía y heterogeneidad hidrolitológica, parece evidenciarse una disminución de la permeabilidad hacia el Este, conforme predominan proporcionalmente texturas más finas.

Mucho más regulares son las cuantías del Coeficiente de Almacenamiento (S), oscilando entre $10^{-4}$ a $10^{-6}$, con $10^{-5}$ como orden más frecuente, indicando el carácter confinado de la Unidad Cretácica acuífera. 
Un caso aparte es el del método de Papadópulos-Cooper, con un valor de $\boldsymbol{T}$ de $12 \mathrm{~m}^{2} / \mathrm{d}$ pero del coeficiente de Almacenamiento con un orden similar a una capa freática $\left(8 \times 10^{-2}\right)$, efecto común en ese tipo de captaciones de gran diámetro, donde prevalece la influencia de la porosidad efectiva por sobre los efectos devenidos de la elasticidad del esqueleto acuífero y de la propia agua.

Todos estos ensayos descriptos están referidos exclusivamente a la Unidad Cretácica acuífera, ya que por sus características constructivas no pudieron concretarse en las obras de pobladores locales que captan el acuífero freático, generalmente en el valle del Arroyo Perdido y cañadones...."
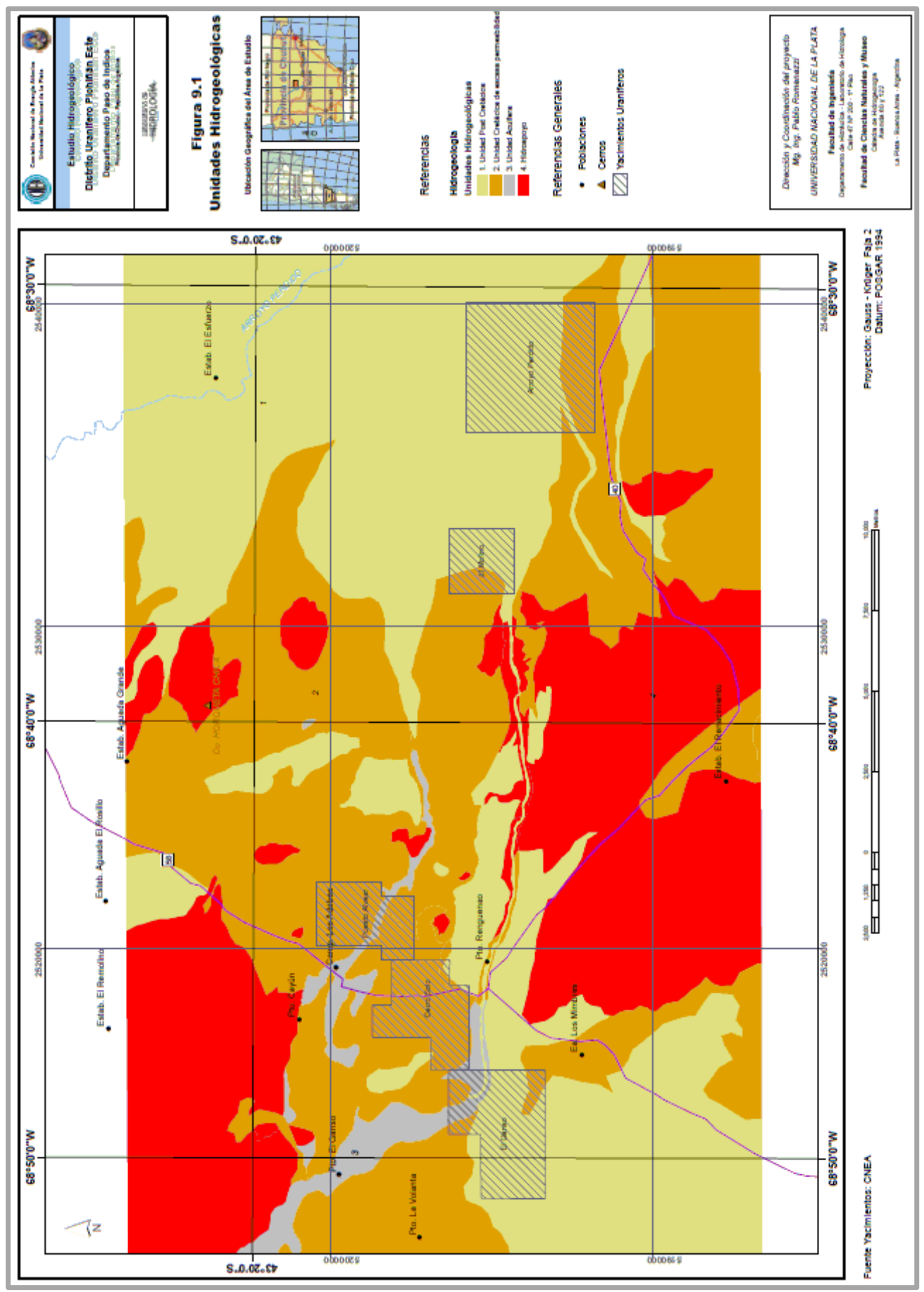

Figura 8.12: Unidades Hidrogeológicas y ubicación de yacimientos (Hernández et al. 2012). 


\subsection{ARCHIVOS DE DATOS Y PROGRAMAS AUXILIARES}

\section{Ejemplo de archivo de datos para la generación de fracturas con Ellipsim}

\begin{tabular}{|c|c|}
\hline \multicolumn{2}{|c|}{$\begin{array}{l}\text { Parameters for ELLIPSIM } \\
* * * * * * * * * * * * * * * * * * * * *\end{array}$} \\
\hline TART OF PARAMETERS: & \\
\hline e5p3f100.out & -file for output realizations \\
\hline 100 & -number of realizations \\
\hline 1020.1 .0 & $-n x, x m n, x \operatorname{siz}$ \\
\hline 1020.1 .0 & -ny,ymn,ysiz \\
\hline 520.1 .0 & -nz,zmn,zsiz \\
\hline 30061960 & -random number seed \\
\hline 0.05 & -target proportion (in ellipsoids) \\
\hline 2000.00 .52000 .090 .00 .00 .01 .0 & -radius $[1,2,3]$, angle $[1,2,3]$, weight \\
\hline 2000.00 .52000 .00 .090 .00 .01 .0 & $-\operatorname{radius}[1,2,3]$, angle $[1,2,3]$, weight \\
\hline 2000.00 .52000 .00 .00 .090 .01 .0 & - radius $[1,2,3]$, angle $[1,2,3]$, weight \\
\hline
\end{tabular}

\section{Ejemplo de código Fortran 95 para cálculo de intercaras usando resultados de SISIM para tres familias de fracturas.}

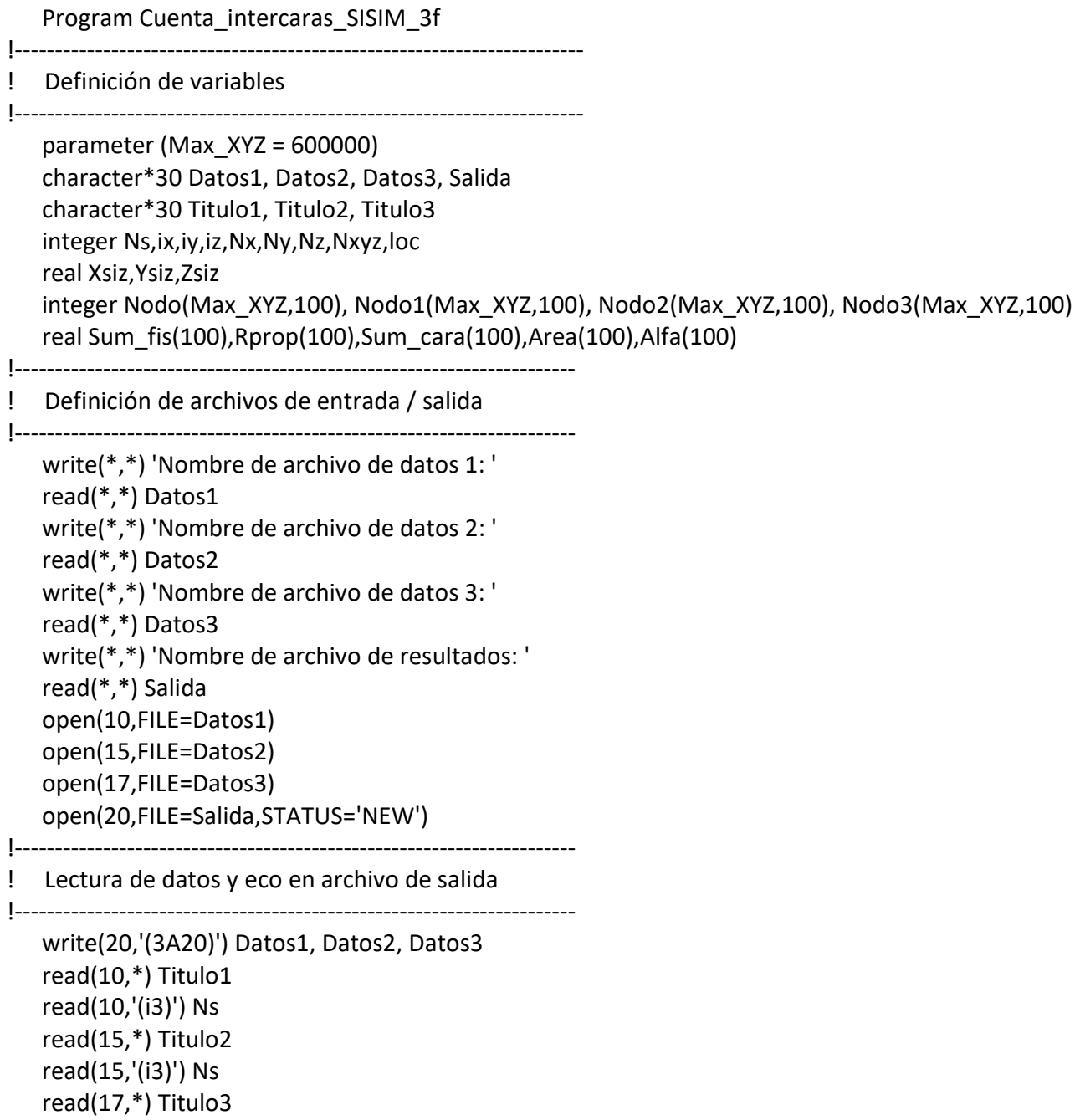




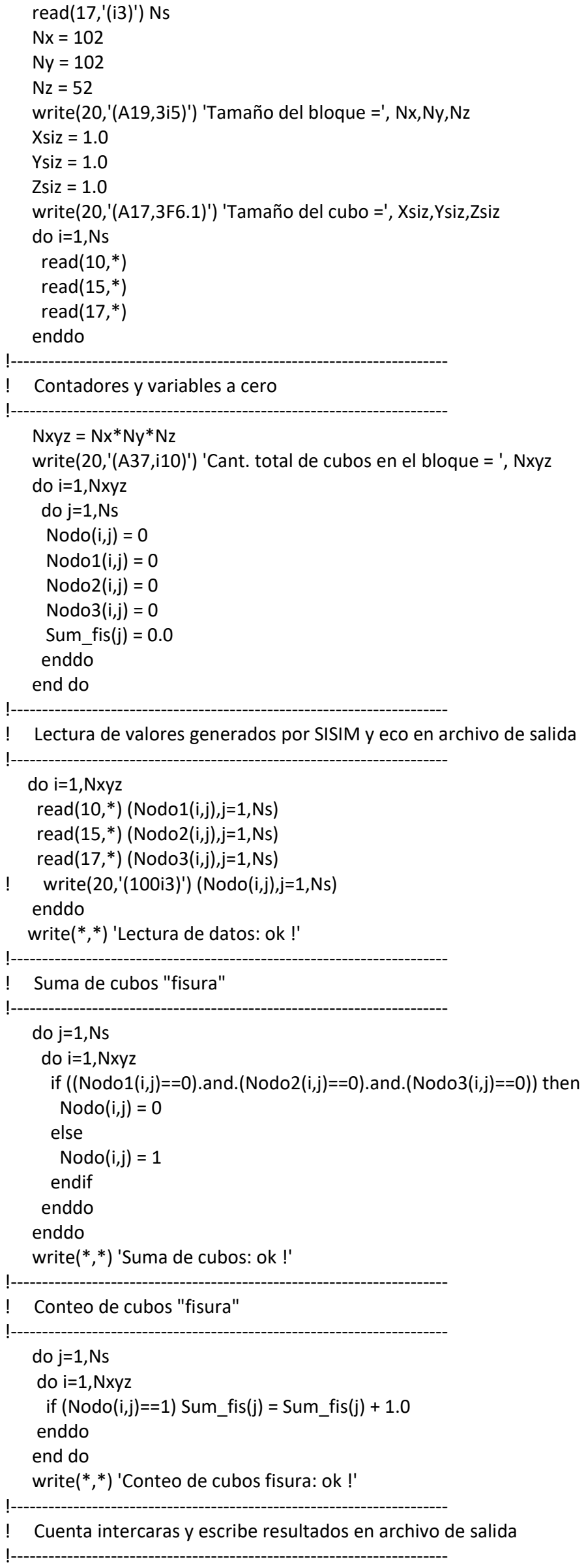




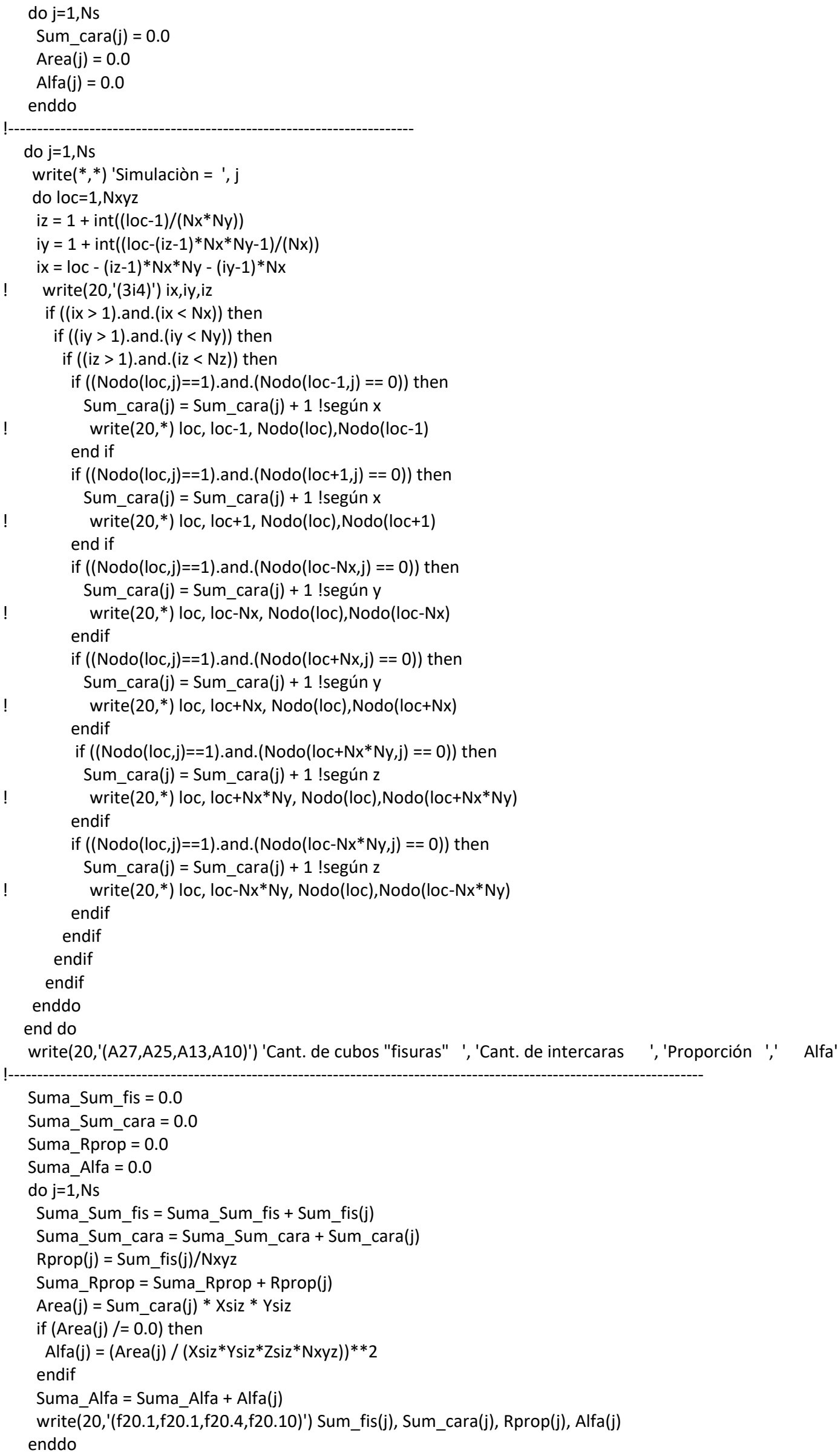


write $(20, *)$

write(20,'(f20.1,f20.1,f20.4,f20.10)') Suma_Sum_fis/Ns, Suma_Sum_cara/Ns, Suma_Rprop/Ns, Suma_Alfa/Ns

! Cerrar archivos y finalizar programa

close(10)

close(15)

close(17)

close (20)

write $(*, *)$ 'Programa finalizado correctamente'

end 